\title{
Daisy Chain Dendrimers: Integrated Mechanically Interlocked Molecules with Stimuli-induced Dimension Modulation Feature
}

Wei-Jian $\mathrm{Li}^{\dagger}$, Wei Wang ${ }^{\dagger}, *$, Xu-Qing Wang ${ }^{\dagger}, \mathrm{Mu} \mathrm{Li}^{\S}$, Yubin $\mathrm{Ke}^{\ddagger}$, Rui Yao ${ }^{\dagger}$, Jin Wen ${ }^{\wedge}$, Guang-Qiang Yin ${ }^{\dagger, \#}$, Bo Jiang ${ }^{\dagger}$, Xiaopeng Li ${ }^{\#}$, Panchao Yin ${ }^{\S}$, Hai-Bo Yang ${ }^{\dagger, *}$

†Shanghai Key Laboratory of Green Chemistry and Chemical Processes \& Chang-Kung Chuang Institute, School of Chemistry and Molecular Engineering, East China Normal University, 3663 N. Zhongshan Road, Shanghai 200062, P. R. China.

${ }^{\S}$ South China Advanced Institute for Soft Matter Science and Technology \& State Key Laboratory of Luminescent Materials and Devices, South China University of Technology, Guangzhou, 510640 P. R. China.

${ }^{\ddagger}$ Spallation Neutron Source Science Center, Dongguan 523803, China

^Institute of Theoretical Chemistry, Faculty of Vienna, University of Vienna, Währinger Str. 17, A-1090 Vienna, Austria

\#Department of Chemistry, University of South Florida, Tampa, Florida 33620, United States.

*To whom correspondence should be addressed:

E-mail: wwang@ chem.ecnu.edu.cn (W. Wang); jin.wen@univie.ac.at (J. Wen); hbyang@chem.ecnu.edu.cn (H.-B. Yang) 
Table of Contents (93 Pages)

Section 1. Supplementary materials and methods

Section 2. Synthesis and characterization of the [c2]daisy chain rotaxanes 1 and 2 . ..S4

Section 3. Synthesis and characterization of the daisy chain dendrimers DC-Gn $(\mathrm{n}=1,2,3)$.

1. Synthesis of the daisy chain dendrimers $\mathbf{D C}-\mathbf{G n}$ S18

2. NMR, MS and GPC spectra of the daisy chain dendrimers $\mathbf{D C}-\boldsymbol{G n}$ S24

3. 2-D DOSY spectra of the daisy chain dendrimers DC-Gn... S36

4. AFM images for the daisy chain dendrimers $\mathbf{D C}-\mathbf{G n}$. S37

5. UV-vis spectra of the daisy chain dendrimers $\mathbf{D C}-\mathbf{G n}$ .$S 38$

Section 4. Anion- and solvent-induced switching of the [c2]daisy chain rotaxane 2 . S40

Section 5. Anion- and solvent-induced dimension modulation of the daisy chain dendrimers S50

Section 6. Synthesis and characterization of the model dendrimer .$S 66$

Section 7. Solvent-induced shape transformation behaviors of DC-Gn@PVDF films S76

Section 8. Small angle neutron scattering (SANS) experiments. S79

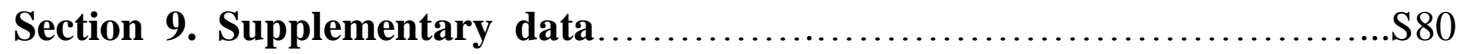

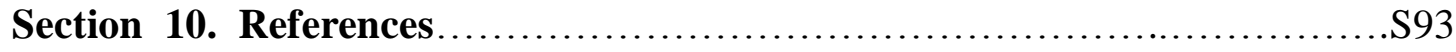




\section{Section 1. Supplementary materials and methods}

All reagents were commercially available and used as supplied without further purification, compounds $\mathbf{S}_{2}-\mathbf{S}_{\mathbf{8}}$ were prepared according to the published procedures $^{\mathrm{S} 1 \mathrm{~S} 4}$. Deuterated solvents were purchased from Cambridge Isotope Laboratory (Andover, MA).

All solvents were dried according to standard procedures and all of them were degassed under $\mathrm{N}_{2}$ for 30 minutes before use. All air-sensitive reactions were carried out under inert $\mathrm{N}_{2}$ atmosphere. ${ }^{1} \mathrm{H}$ NMR, ${ }^{13} \mathrm{C}$ NMR and ${ }^{31} \mathrm{P}$ NMR spectra were recorded on Bruker $500 \mathrm{MHz}$ Spectrometer $\left({ }^{1} \mathrm{H}: 500 \mathrm{MHz} ;{ }^{31} \mathrm{P}: 202 \mathrm{MHz} ;{ }^{13} \mathrm{C}: 126\right.$ $\mathrm{MHz})$ at $298 \mathrm{~K}$. The ${ }^{1} \mathrm{H}$ and ${ }^{13} \mathrm{C}$ NMR chemical shifts are reported relative to residual solvent signals, and ${ }^{31} \mathrm{P}\left\{{ }^{1} \mathrm{H}\right\}$ NMR chemical shifts are referenced to an external unlocked sample of $85 \% \mathrm{H}_{3} \mathrm{PO}_{4}(\delta 0.0)$. 2D NMR spectra $\left({ }^{1}{ }^{\mathrm{H}}-{ }^{1} \mathrm{H}\right.$ COSY, ROESY and DOSY) were recorded on Bruker $500 \mathrm{MHz}$ Spectrometer $\left({ }^{1} \mathrm{H}: 500 \mathrm{MHz}\right)$ at $298 \mathrm{~K}$. DLS measurements were performed under a Malvern Zetasizer Nano-ZS light scattering apparatus (Malvern Instruments, U.K.) with a He-Ne laser (633 nm, 4 mW). The MALDI MS experiments were carried out on a Bruker UltrafleXtreme MALDI TOF/TOF Mass Spectrometer (Bruker Daltonics, Billerica, MA), equipped with smartbeam-II laser. Electrospray ionization (ESI) mass spectra were recorded with a Waters Synapt G2 mass spectrometer. All spectra were measured in positive reflectron or linear mode. All the AFM images were obtained on a Dimension FastScan (Bruker), using ScanAsyst mode under ambient condition. For the AFM samples, the solution of the daisy chain dendrimers DC-Gn $(n=1,2,3)$ in THF $\left(c=10^{-7} \mathrm{M}\right)$ was firstly prepared. Then the AFM samples were obtained by drop casting method using mica sheet as substrate. UV-vis spectra were recorded in a quartz cell (light path $10 \mathrm{~mm}$ ) on a Shimadzu UV2700 UV-Visible spectrophotometer. 
Section 2. Synthesis and characterization of the [c2]daisy chain

rotaxane 1 and 2

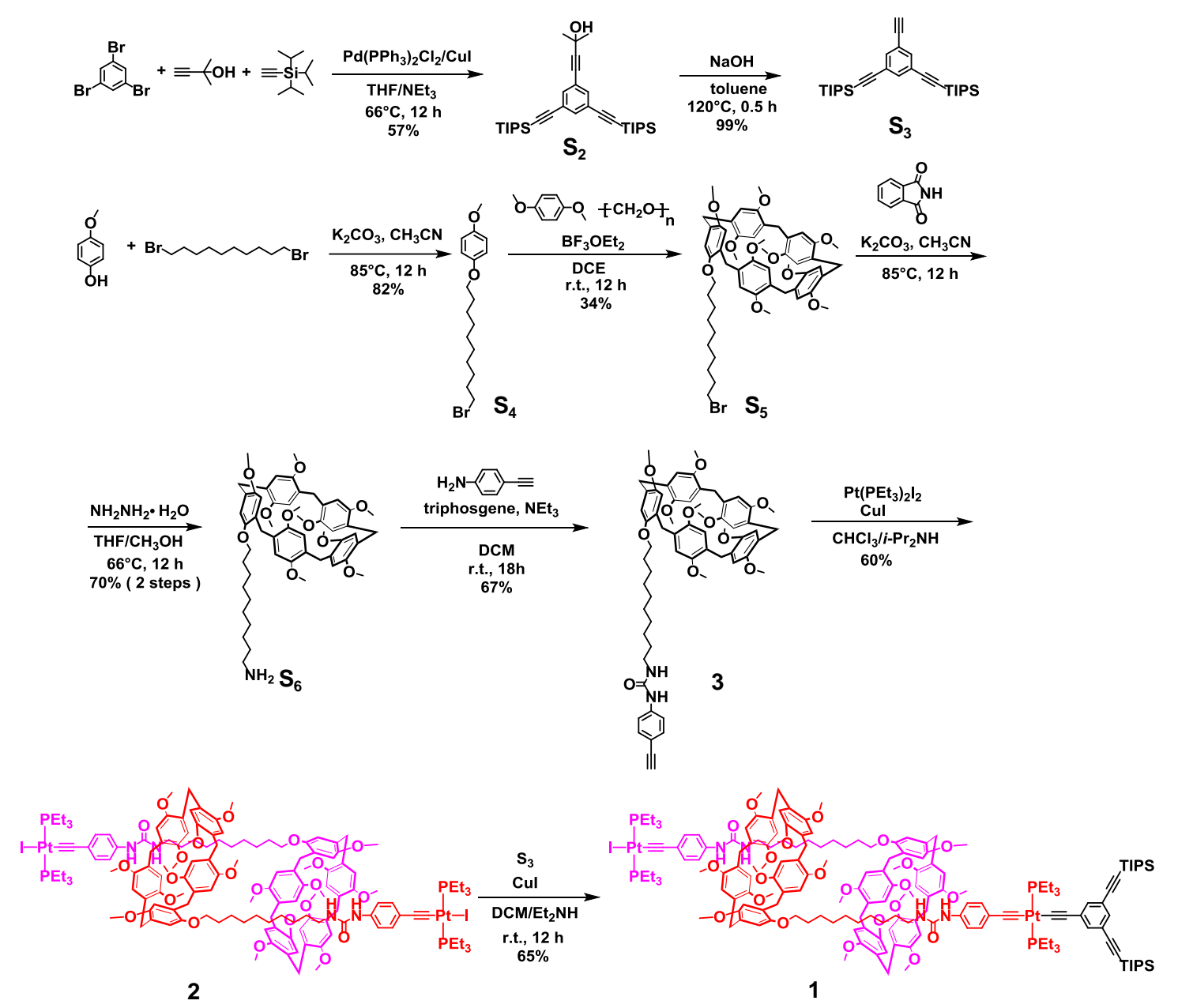

Scheme S1: The synthesis route of [c2]daisy chain rotaxane 1-2.

Synthesis of 3: $\mathrm{A} \mathrm{CH}_{2} \mathrm{Cl}_{2}(25 \mathrm{~mL})$ solution of triphosgene (420 mg, $\left.1.42 \mathrm{mmol}\right)$ was added into a Schlenk flask, the Schlenk flask was evacuated and back-filled with $\mathrm{N}_{2}$ three times. Then a $\mathrm{CH}_{2} \mathrm{Cl}_{2}(25 \mathrm{~mL})$ solution of 4-Ethynylaniline $(500 \mathrm{mg}, 4.27 \mathrm{mmol})$ and triethylamine $(2.4 \mathrm{~mL})$ were added into the reaction flask and stirred at $0{ }^{\circ} \mathrm{C}$. After $4 \mathrm{~h}$, adding the $\mathrm{CH}_{2} \mathrm{Cl}_{2}(25 \mathrm{~mL})$ solution of $\mathbf{S}_{\mathbf{6}}(3.81 \mathrm{~g}, 4.27 \mathrm{mmol})$ into reaction flask, then the reaction mixture was allowed to warm to room temperature and stirred overnight. The solution was concentrated and the residue was purified through column chromatography $\left(\mathrm{SiO}_{2}\right.$; DCM/EA) to afford compound $\mathbf{3}$ as a pale-yellow solid (2.89 g, 65\% yield). ${ }^{1} \mathrm{H}$ NMR (500 MHz, $\left.\mathrm{CD}_{2} \mathrm{Cl}_{2}\right): \delta 7.38-7.39(\mathrm{~m}, 2 \mathrm{H})$, 7.31-7.32 (m, 2H), 6.85-6.88 (m, 10H), $6.59(\mathrm{~s}, 1 \mathrm{H}), 4.72(\mathrm{~m}, 1 \mathrm{H}), 3.85-3.88(\mathrm{t}, J=$ $7.5 \mathrm{~Hz}, 2 \mathrm{H}), 3.69-3.75(\mathrm{~m}, 37 \mathrm{H}), 3.13(\mathrm{~m}, 2 \mathrm{H}), 3.06(\mathrm{~s}, 1 \mathrm{H}), 1.81-1.84(\mathrm{~m}, 2 \mathrm{H})$, 1.26-1.39(m, 14H). ${ }^{13} \mathrm{C}$ NMR (126 MHz, $\left.\mathrm{CD}_{2} \mathrm{Cl}_{2}\right): \delta 156.32,151.93,151.90,151.81$, 
$151.30,141.66,134.43,129.95,129.85,129.82,129.79,129.76,129.72,129.68$, 129.66, 120.30, 117.28, 115.69, 114.91, 114.85, 114.81, 114.73, 85.04, 77.65, 69.84, 57.07, 57.01, 56.91, 41.92, 31.55, 31.39, 30.98, 30.88, 30.80, 30.72, 30.68, 30.55, 30.47, 28.38, 27.79. HRMS (MALDI-TOF-MS): Calculated for $[\mathbf{3}+\mathrm{Na}]^{+}: m / z=$ 1057.5185; Found: $m / z=1057.5154$. Calculated for $[3+\mathrm{K}]^{+}: m / z=1073.4924$; Found: $m / z=1073.4885$.

Synthesis of 2: A Schlenk flask was charged with $400 \mathrm{mg}(0.39 \mathrm{mmol})$ of 3 and $\mathrm{Pt}\left(\mathrm{PEt}_{3}\right)_{2} \mathrm{I}_{2}(1.32 \mathrm{~g}, 1.92 \mathrm{mmol})$. The Schlenk flask was then evacuated and back-filled with $\mathrm{N}_{2}$ three times. Next, $6 \mathrm{~mL}$ of the mixed solvent of anhydrous and degassed $\mathrm{CHCl}_{3}$ and $i-\mathrm{Pr}_{2} \mathrm{NH}(\mathrm{v} / \mathrm{v}, 2: 1)$ was added via syringe. After stirring at $-35{ }^{\circ} \mathrm{C}$ for 24 hours, $\mathrm{CuI}(16 \mathrm{mg})$ was added to the mixture under an inert atmosphere and the mixture was stirred for 48 hours under $-35{ }^{\circ} \mathrm{C}$ continuously. Then the mixture was allowed to warm to room temperature and stirred for 48 hours. The solution was concentrated and the residue was purified by column chromatography $\left(\mathrm{SiO}_{2}\right.$; DCM/EA) and gel permeation chromatography (GPC). A pale yellow solid 2 (372 $\mathrm{mg}$, $60 \%$ yield) was obtained. ${ }^{1} \mathrm{H}$ NMR $\left(500 \mathrm{MHz}, \mathrm{CD}_{2} \mathrm{Cl}_{2}\right): \delta 7.30-7.31(\mathrm{~m}, 4 \mathrm{H})$, 7.22-7.24 (m, 4H), 6.96-7.01 (m, 12H), 6.85-6.90 (m, 10H), 3.74-3.84 (m, 78H), 2.72-2.74 (m, 2H), 2.22-2.27 (m, 24H), 1.91-2.26 (m, 4H), $1.53(\mathrm{~m}, 4 \mathrm{H}), 1.41(\mathrm{~m}, 4 \mathrm{H})$, 1.15-1.21 (m, 36H), $0.87(\mathrm{~m}, 4 \mathrm{H}), 0.59(\mathrm{~m}, 4 \mathrm{H}),-0.26(\mathrm{~m}, 4 \mathrm{H}),-2.00-(-1.89)(\mathrm{m}, 4 \mathrm{H})$, -2.25- (-2.18) (m, 4H). ${ }^{31} \mathrm{P}$ NMR (202 MHz, $\left.\mathrm{CD}_{2} \mathrm{Cl}_{2}\right): \delta 8.84 .{ }^{13} \mathrm{C} \mathrm{NMR} \mathrm{(126} \mathrm{MHz,}$ $\left.\mathrm{CD}_{2} \mathrm{Cl}_{2}\right): \delta 153.23,153.16,150.03,149.94,149.85,149.79,149.77,149.71,149.68$, $149.62,149.58,149.53,149.50,149.44,137.35,137.21,130.38,130.21,128.24$, $128.09,128.05,128.03,127.97,127.90,127.85,127.79,127.70,127.57,127.55$, $127.51,127.48,127.46,127.35,127.30,121.26,118.13,114.05,113.86,113.74$, $113.62,113.58,113.54,113.45,113.32,113.19,112.57,112.48,112.41,112.31$, $112.27,112.10,112.05,99.02,86.76,86.64,86.53,68.11,67.86,55.99,55.77,55.69$, $55.64,55.59,55.46,55.36,55.31,54.62,54.53,54.51,54.46,54.43,54.33,54.32$, $39.01,38.80,30.78,30.46,30.06,29.97,29.91,29.75,29.58,29.31,29.09,28.91$, $28.38,28.32,28.27,28.20,28.14,26.39,26.22,25.62,25.19,23.80,23.74,15.99$, 15.85, 15.71, 13.92, 13.78, 13.64, 7.32, 7.07. LRMS (MALDI-TOF-MS): Calculated for $[2+\mathrm{H}]^{+}: m / z=3184.2$; Found: $m / z=3184.1$. HRMS (ESI-TOF-MS): Calculated for $[2+2 \mathrm{H}]^{2+}: m / z=1593.0790$; Found: $m / z=1593.0790$. Anal. Calcd. for $\mathrm{C}_{150} \mathrm{H}_{206} \mathrm{I}_{2} \mathrm{~N}_{4} \mathrm{O}_{22} \mathrm{P}_{4} \mathrm{Pt}_{2}$ : C, 56.56; H, 6.52; N, 1.76, Found: C, 56.71; H, 6.67; N, 1.60. 


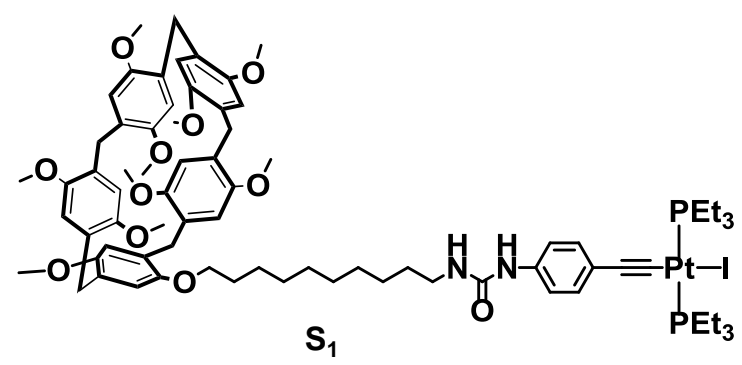

Figure S1: Chemical structure of the non-interlocked sole axle compound $\mathbf{S}_{\mathbf{1}}$.

In the procedure for the synthesis of $\mathbf{2}$, the non-interlocked compound $\mathbf{S}_{\mathbf{1}}$ was also isolated $(150 \mathrm{mg}) .{ }^{1} \mathrm{H}$ NMR $\left(500 \mathrm{MHz}, \mathrm{CD}_{2} \mathrm{Cl}_{2}\right): \delta 7.21-7.25(\mathrm{~m}, 4 \mathrm{H}), 6.89-6.93(\mathrm{~m}$, $10 \mathrm{H}), 6.27(\mathrm{~s}, 1 \mathrm{H}), 4.59(\mathrm{~m}, 1 \mathrm{H}), 3.90-3.92(\mathrm{t}, J=5.0 \mathrm{~Hz}, 2 \mathrm{H}), 3.74-3.79(\mathrm{~m}, 37 \mathrm{H})$, 3.17-3.21 (m, 2H), 2.23-2.26 (m, 12H), 1.84-1.90 (m, 2H), 1.57-1.60 (m, 4H), $1.39-1.46$ (m, 10H), 1.16-1.23 (m, 18H). ${ }^{31} \mathrm{P}$ NMR (202 MHz, $\left.\mathrm{CD}_{2} \mathrm{Cl}_{2}\right): \delta 8.94 .{ }^{13} \mathrm{C}$ NMR (126 MHz, $\left.\mathrm{CD}_{2} \mathrm{Cl}_{2}\right) \delta 156.70,151.92,151.91,151.80,151.29,138.17,132.76$, $129.92,129.85,129.80,129.77,129.73,129.71,129.68,129.64,125.09,121.63$, $115.67,114.88,114.83,114.80,114.71,90.05,69.84,57.05,56.99,56.98,56.90$, $41.96,33.50,31.64,31.41,31.26,31.22,31.05,31.02,31.00,30.93,30.90,30.79$, $30.71,30.68,30.55,30.46,28.42$, 27.82, 24.26, 18.29, 18.15, 18.01, 15.45, 9.61. HRMS (ESI-TOF-MS): Calculated for $\left[\mathbf{S}_{\mathbf{1}}+\mathrm{H}\right]^{+}: m / z=1593.5865$; Found: $m / z=$ 1593.5865 .

Synthesis of 1: A Schlenk flask was charge with $\mathbf{S} 3$ (262 $\mathrm{mg}, 0.56 \mathrm{mmol}$ ) and compound 2 ( $3.64 \mathrm{~g}, 1.14 \mathrm{mmol})$. The Schlenk flask was then evacuated via reduced pressure and backfilled with $\mathrm{N}_{2}$ for three times. Next, dichloromethane $(80 \mathrm{~mL})$ and diethylamine $(40 \mathrm{~mL})$ was added via syringe. The resultant solution was stirred for 2 hours at room temperature. Then $\mathrm{CuI}(24 \mathrm{mg})$ was added to the mixture under $\mathrm{N}_{2}$ atmosphere, and the mixture was allowed to stir for 12 hours. The solvent was then removed by reduced pressure, and the compound was purified by column chromatography $\left(\mathrm{SiO}_{2} ; \mathrm{DCM} / \mathrm{EA}\right)$ and gel permeation chromatography (GPC). A pale yellow solid 1 (1.31 g, 65\% yield) was obtained. ${ }^{1} \mathrm{H}$ NMR (500 $\left.\mathrm{MHz}, \mathrm{CD}_{2} \mathrm{Cl}_{2}\right): \delta$ 7.31-7.33 (m, 7H), 7.22-7.26 (m, 4H), 6.98-7.02 (m, 12H), 6.87-6.91 (m, 10H), 3.76-3.86 (m, 78H), $2.74(\mathrm{~m}, 2 \mathrm{H}), 2.18-2.27(\mathrm{~m}, 24 \mathrm{H}), 1.94-2.08(\mathrm{~m}, 4 \mathrm{H}), 1.55(\mathrm{~m}$, $4 \mathrm{H}), 1.41(\mathrm{~m}, 4 \mathrm{H}), 1.18-1.26(\mathrm{~m}, 36 \mathrm{H}), 1.15(\mathrm{~s}, 46 \mathrm{H}), 0.87(\mathrm{~m}, 4 \mathrm{H}), 0.61(\mathrm{~m}, 4 \mathrm{H})$, $-0.24(\mathrm{~m}, 4 \mathrm{H}),-1.98-(-1.88)(\mathrm{m}, 4 \mathrm{H}),-2.23-(-2.16)(\mathrm{m}, 4 \mathrm{H}) .{ }^{31} \mathrm{P}$ NMR $(202 \mathrm{MHz}$, $\left.\mathrm{CD}_{2} \mathrm{Cl}_{2}\right): \delta 11.54,8.66 .{ }^{13} \mathrm{C}$ NMR $\left(126 \mathrm{MHz}, \mathrm{CD}_{2} \mathrm{Cl}_{2}\right): \delta$ 154.10, 154.05, 153.98, $150.83,150.74,150.65,150.64,150.58,150.48,150.42,150.37,150.30,138.13$, $137.80,134.05,131.23,131.15,131.00,129.39,129.04,128.89,128.84,128.77$, $128.70,128.64,128.59,128.50,128.37,128.35,128.31,128.28,128.26,128.15$, 
$128.11,123.45,122.49,122.07,118.93,114.86,114.67,114.54,114.43,114.35$, $114.27,114.13,113.98,113.79,113.37,113.28,113.21,113.11,113.07,112.90$, $112.85,111.37,109.19,107.60,106.19,99.82,90.95,87.44,68.92,68.66,39.81$, $39.60,31.95,31.58,31.26,30.86,30.77,30.71,30.54,30.37,30.11,29.89,29.71$, $29.38,29.17,29.11,28.98,27.18,27.02,26.40,25.98,24.59,24.53,22.71,18.55$, $18.44,18.30,16.77,16.63,16.53,16.49,16.39,16.25,14.69,14.56,14.42,13.91$, $11.56,11.45,11.33,11.20,11.11,8.14,8.09,7.84$. LRMS (MALDI-TOF-MS): Calculated for $[\mathbf{1}+\mathrm{H}]^{+}: m / z=3521.1$; Found: $m / z=3521.4$. HRMS (ESI-TOF-MS): Calculated for $[1+2 \mathrm{H}]^{2+}: m / z=1760.7876$; Found: $m / z=1760.7876$. Anal. Calcd. for $\mathrm{C}_{180} \mathrm{H}_{251} \mathrm{IN}_{4} \mathrm{O}_{22} \mathrm{P}_{4} \mathrm{Pt}_{2} \mathrm{Si}_{2}:$ C, 61.42; H, 7.19; N, 1.59, Found: C, 61.25; H, 7.24; N, 1.51.

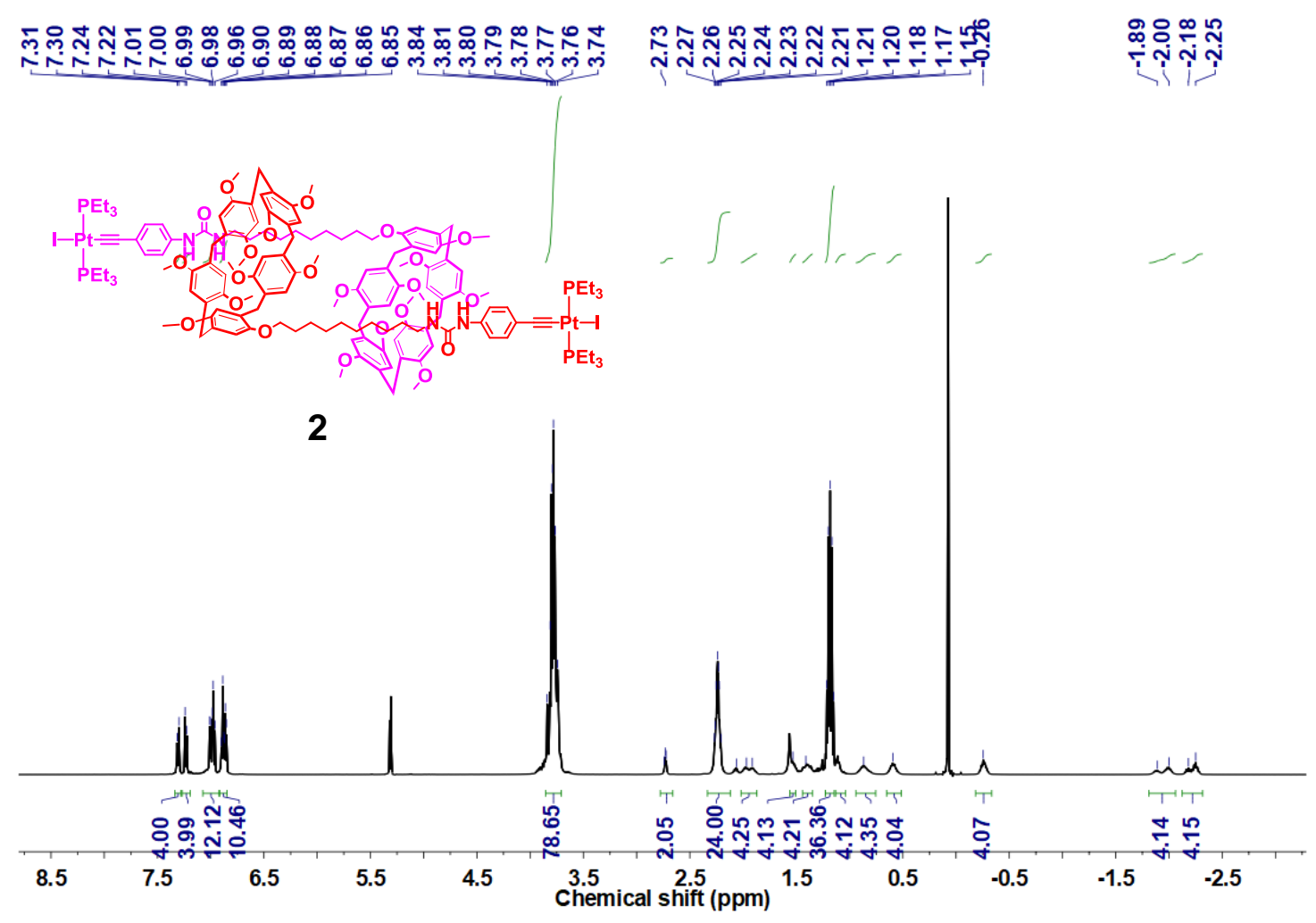

Figure S2: ${ }^{1} \mathrm{H}$ NMR spectrum $\left(\mathrm{CD}_{2} \mathrm{Cl}_{2}, 298 \mathrm{~K}, 500 \mathrm{MHz}\right)$ of the [c2]daisy chain rotaxane 2 . 


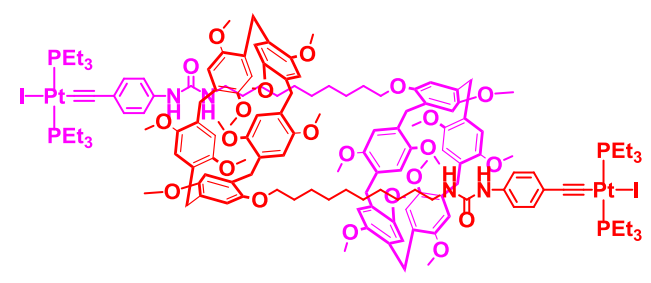

2

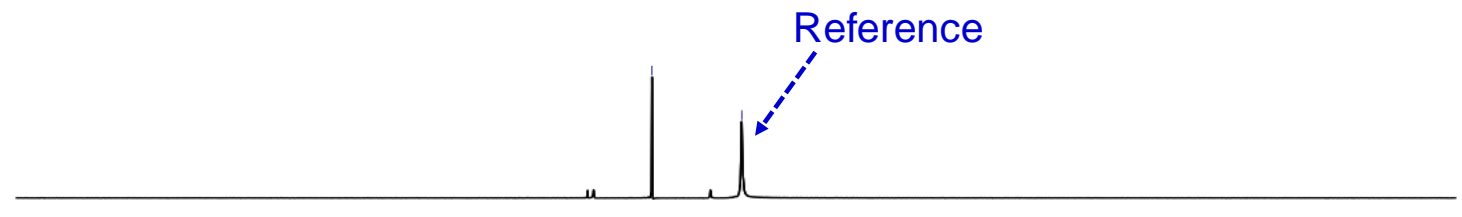

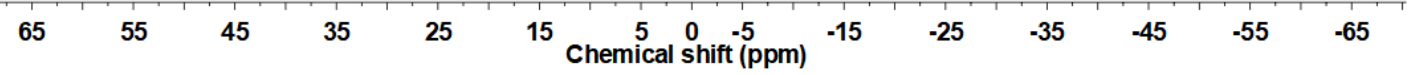

Figure S3: ${ }^{31} \mathrm{P}$ NMR spectrum $\left(\mathrm{CD}_{2} \mathrm{Cl}_{2}, 298 \mathrm{~K}, 202 \mathrm{MHz}\right)$ of the [c2]daisy chain rotaxane 2 .

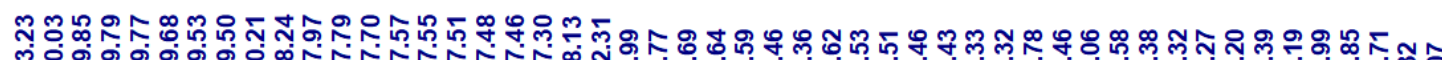

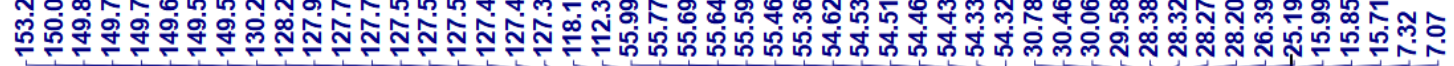

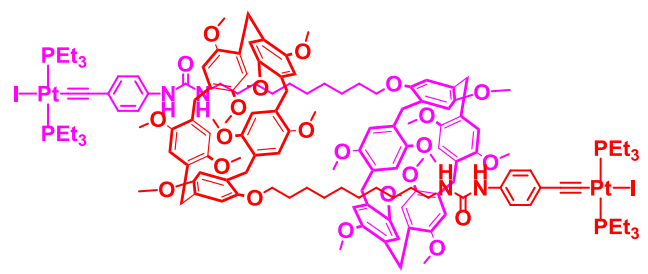

2
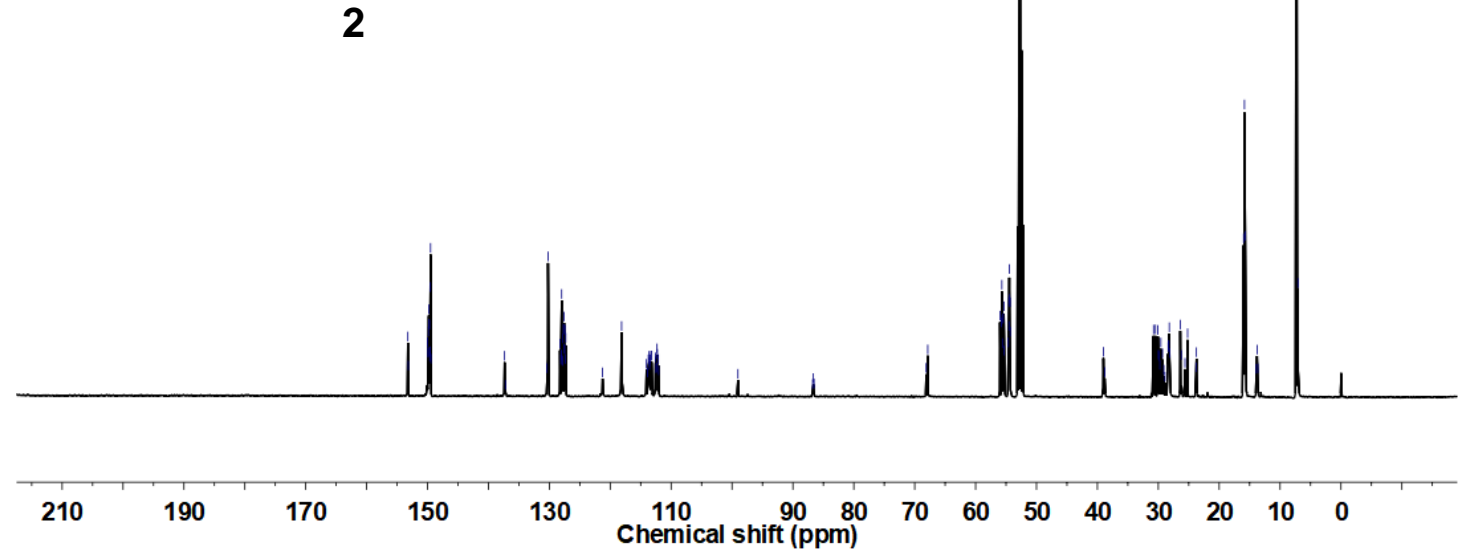

Figure S4: ${ }^{13} \mathrm{C}$ NMR spectrum $\left(\mathrm{CD}_{2} \mathrm{Cl}_{2}, 298 \mathrm{~K}, 126 \mathrm{MHz}\right)$ of the [c2]daisy chain rotaxane 2. 


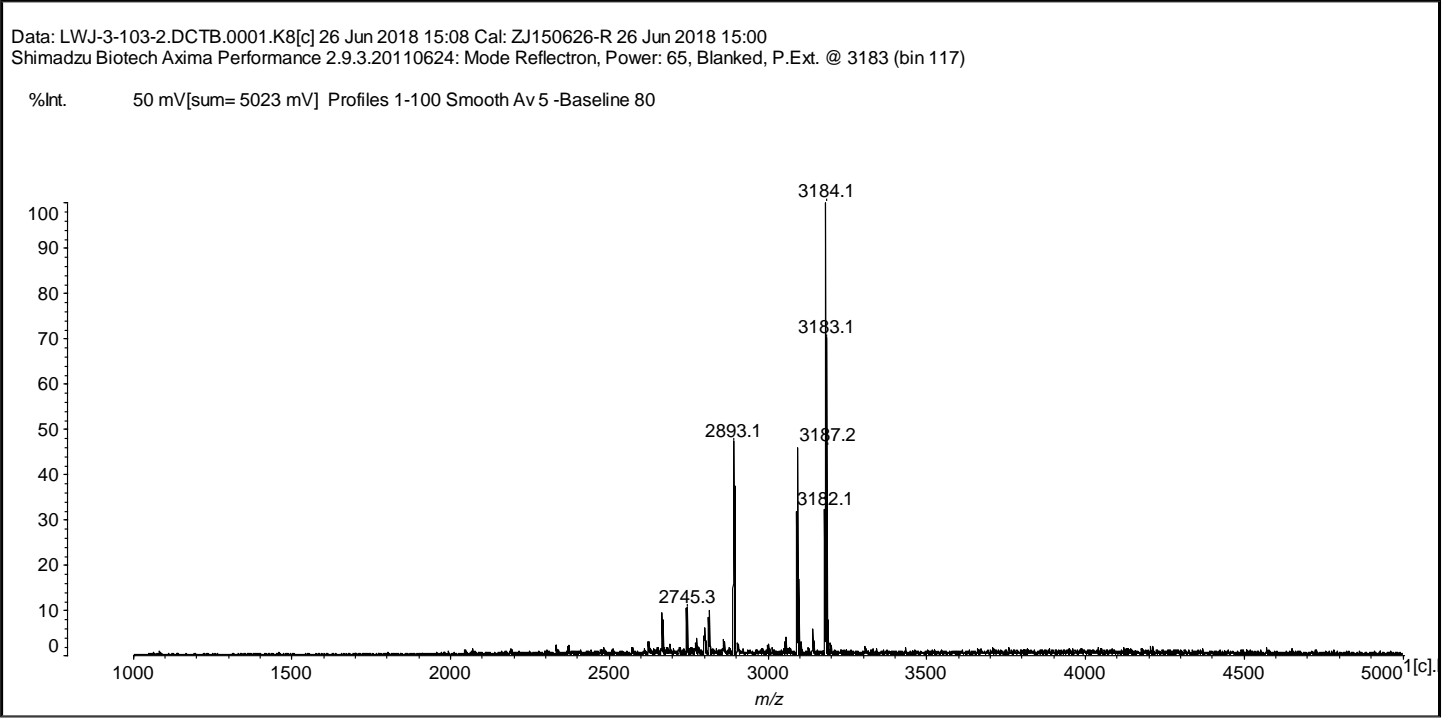

Figure S5: MALDI-TOF-MS spectrum of the [c2]daisy chain rotaxane 2.

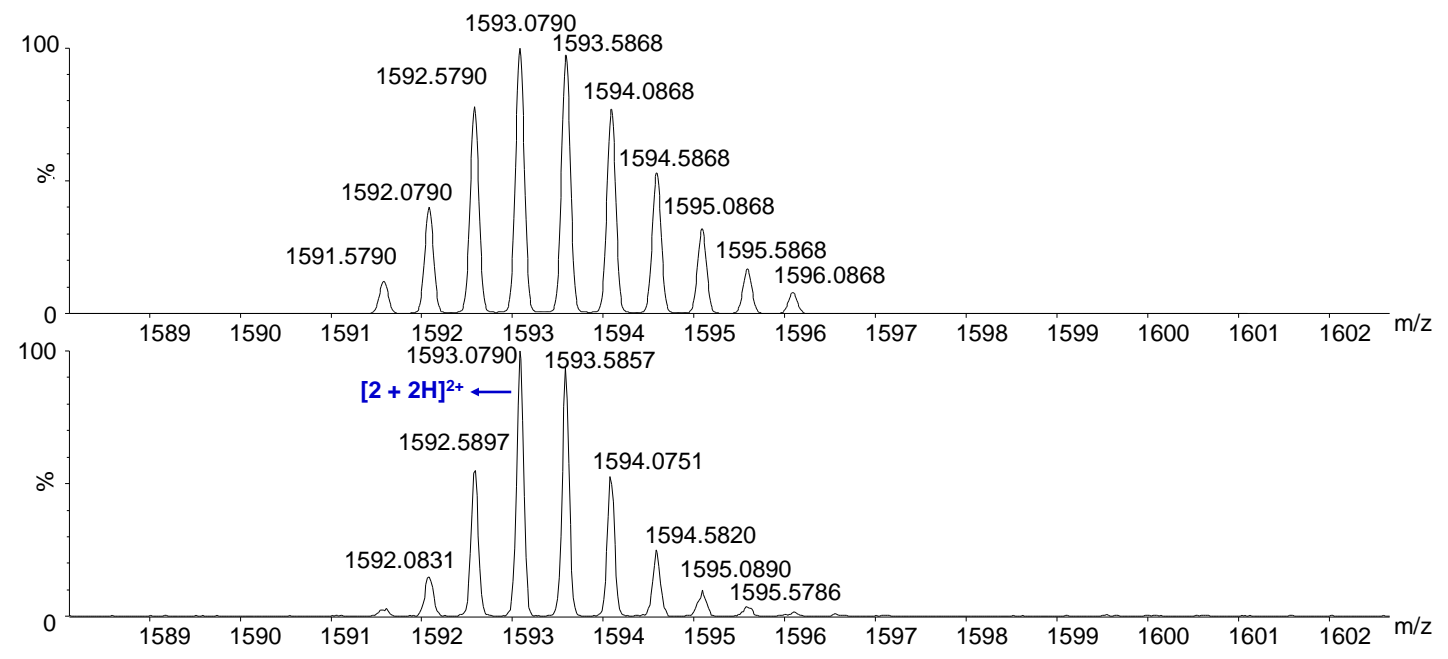

Figure S6: Theoretical (top) and experimental (bottom) ESI-TOF-MS spectra of the [c2]daisy chain rotaxane 2. 


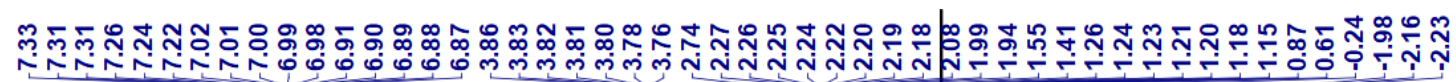

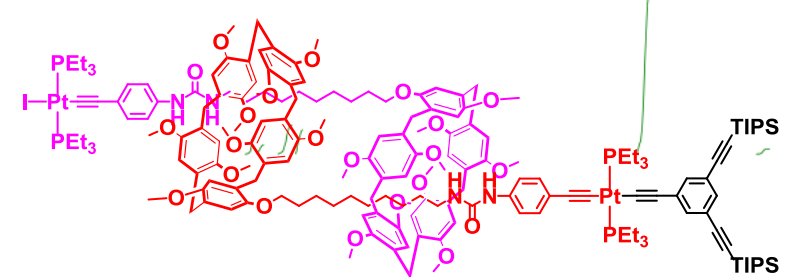

1

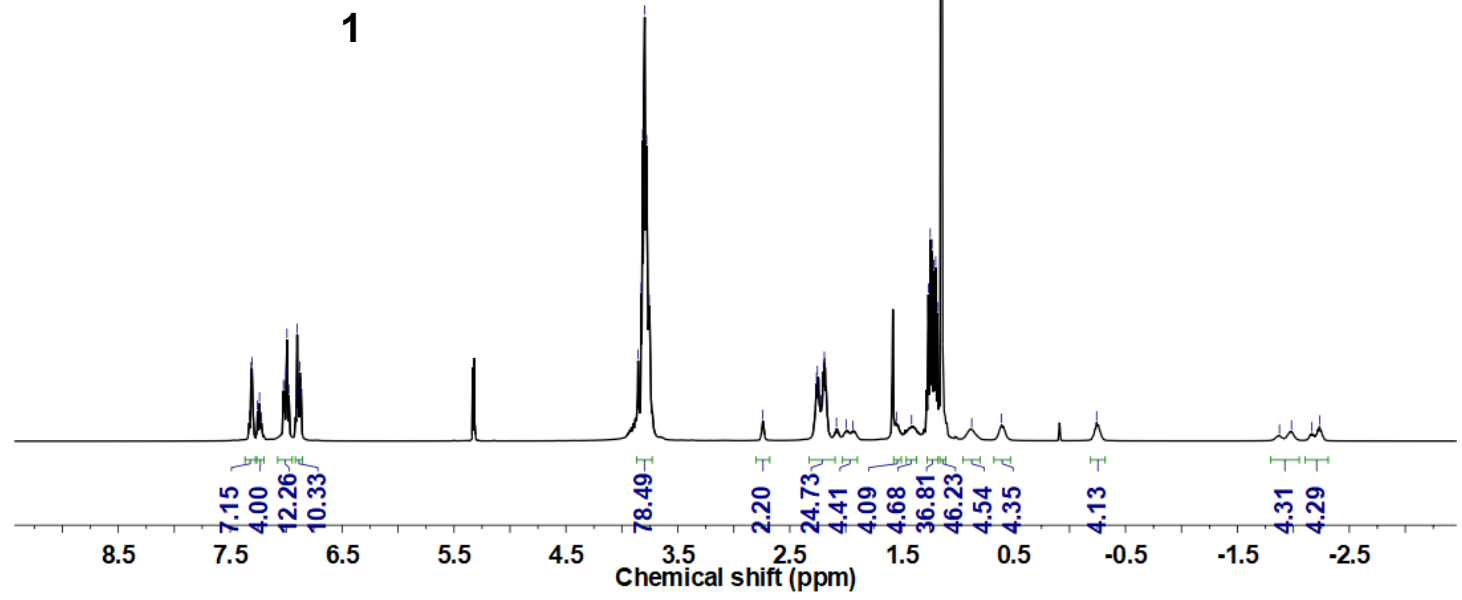

Figure S7: ${ }^{1} \mathrm{H}$ NMR spectrum $\left(\mathrm{CD}_{2} \mathrm{Cl}_{2}, 298 \mathrm{~K}, 500 \mathrm{MHz}\right)$ of the [c2]daisy chain rotaxane 1.

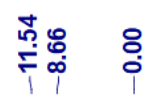

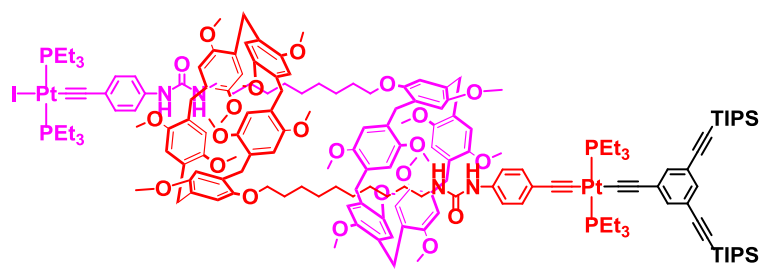

1

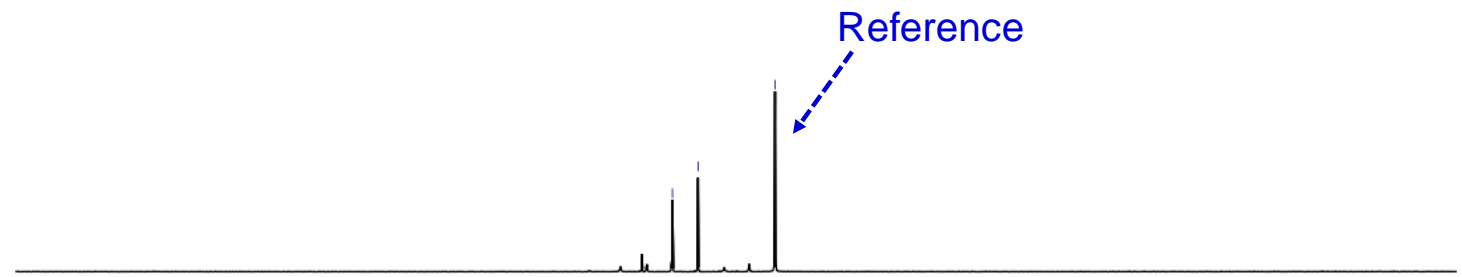

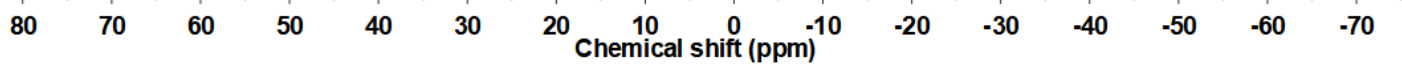

Figure S8: ${ }^{31} \mathrm{P}$ NMR spectrum $\left(\mathrm{CD}_{2} \mathrm{Cl}_{2}, 298 \mathrm{~K}, 202 \mathrm{MHz}\right)$ of the [c2]daisy chain rotaxane 1. 


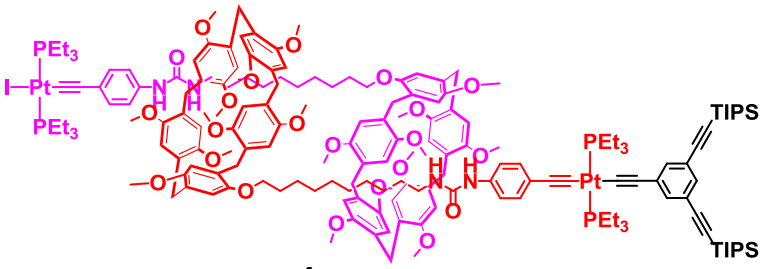

1

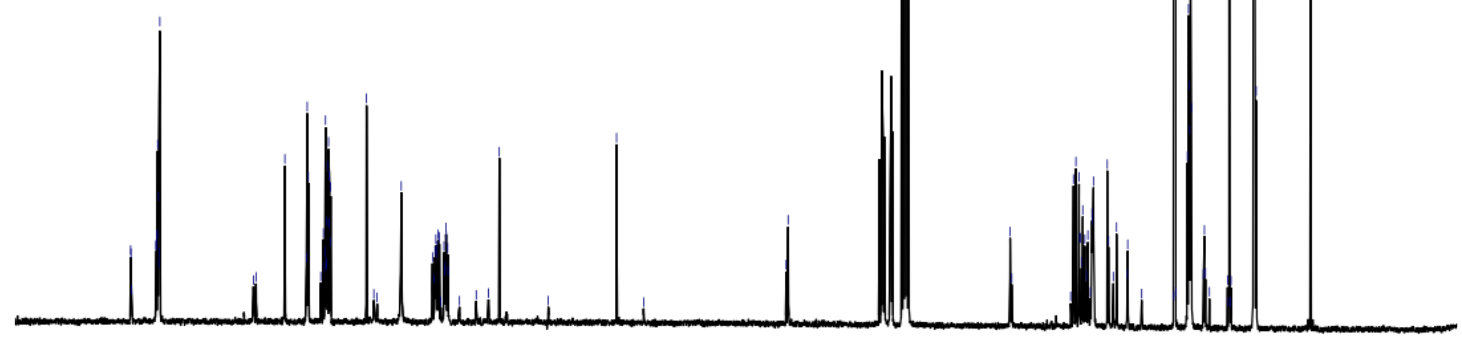

$\begin{array}{llllllllllllllllll}160 & 150 & 140 & 130 & 120 & 110 & 100 & 90 & 80 & 70 & 60 & 50 & 40 & 30 & 20 & 10 & 0 & -10\end{array}$

Figure S9: ${ }^{13} \mathrm{C}$ NMR spectrum $\left(\mathrm{CD}_{2} \mathrm{Cl}_{2}, 298 \mathrm{~K}, 126 \mathrm{MHz}\right)$ of the [c2]daisy chain rotaxane 1.

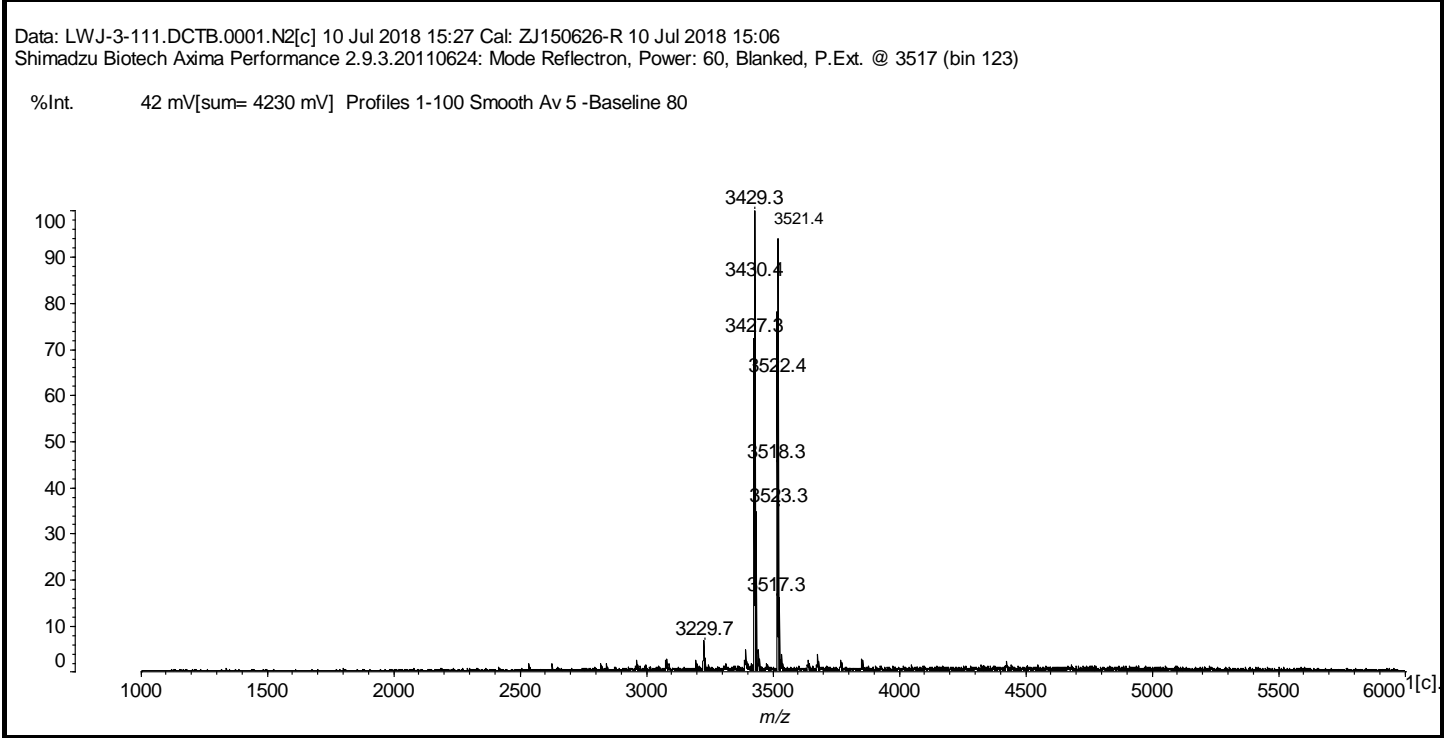

Figure S10: MALDI-TOF-MS spectrum of the [c2]daisy chain rotaxane 1. 


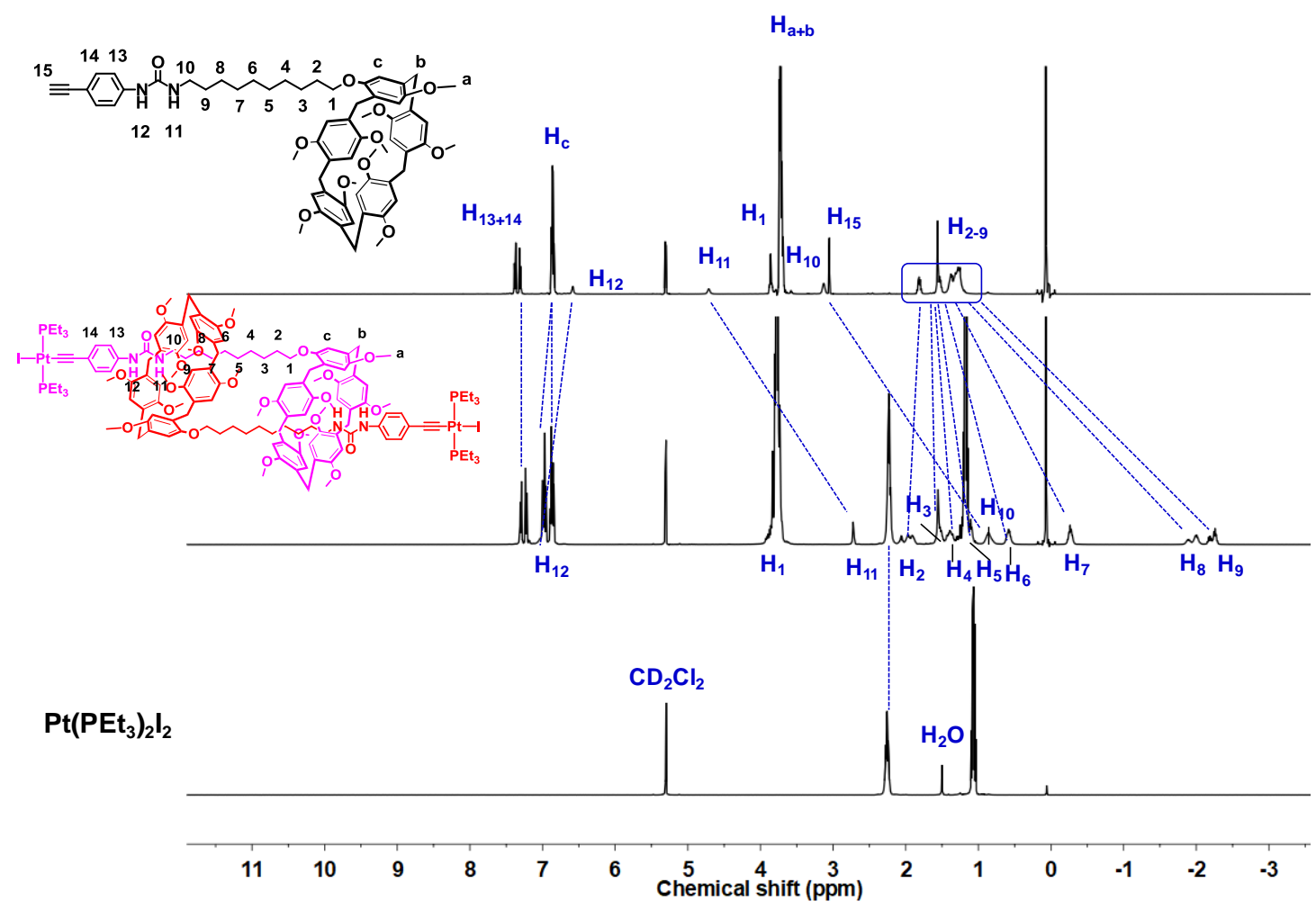

Figure S11: ${ }^{1} \mathrm{H}$ NMR spectra $\left(\mathrm{CD}_{2} \mathrm{Cl}_{2}, 298 \mathrm{~K}, 500 \mathrm{MHz}\right)$ of $\mathrm{Pt}\left(\mathrm{PEt}_{3}\right)_{2} \mathrm{I}_{2}$ (bottom); [c2] daisy chian rotaxane $\mathbf{2}$ (middle); and component $\mathbf{3}$ (top).

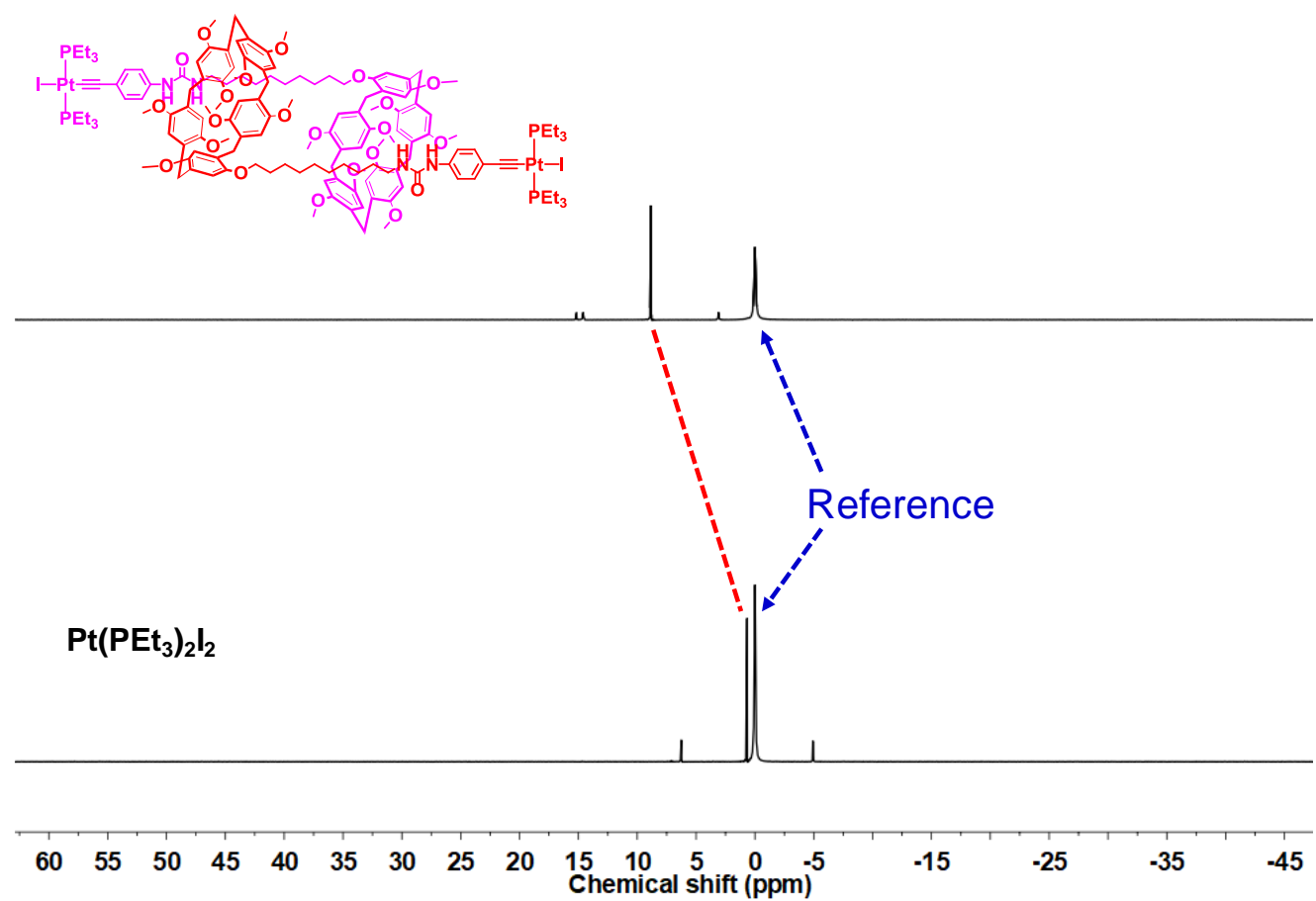

Figure S12: ${ }^{31} \mathrm{P}$ NMR spectra $\left(\mathrm{CD}_{2} \mathrm{Cl}_{2}, 298 \mathrm{~K}, 202 \mathrm{MHz}\right)$ of [c2]daisy chain rotaxane 2 (top) and $\mathrm{Pt}\left(\mathrm{PEt}_{3}\right)_{2} \mathrm{I}_{2}$ (bottom). 


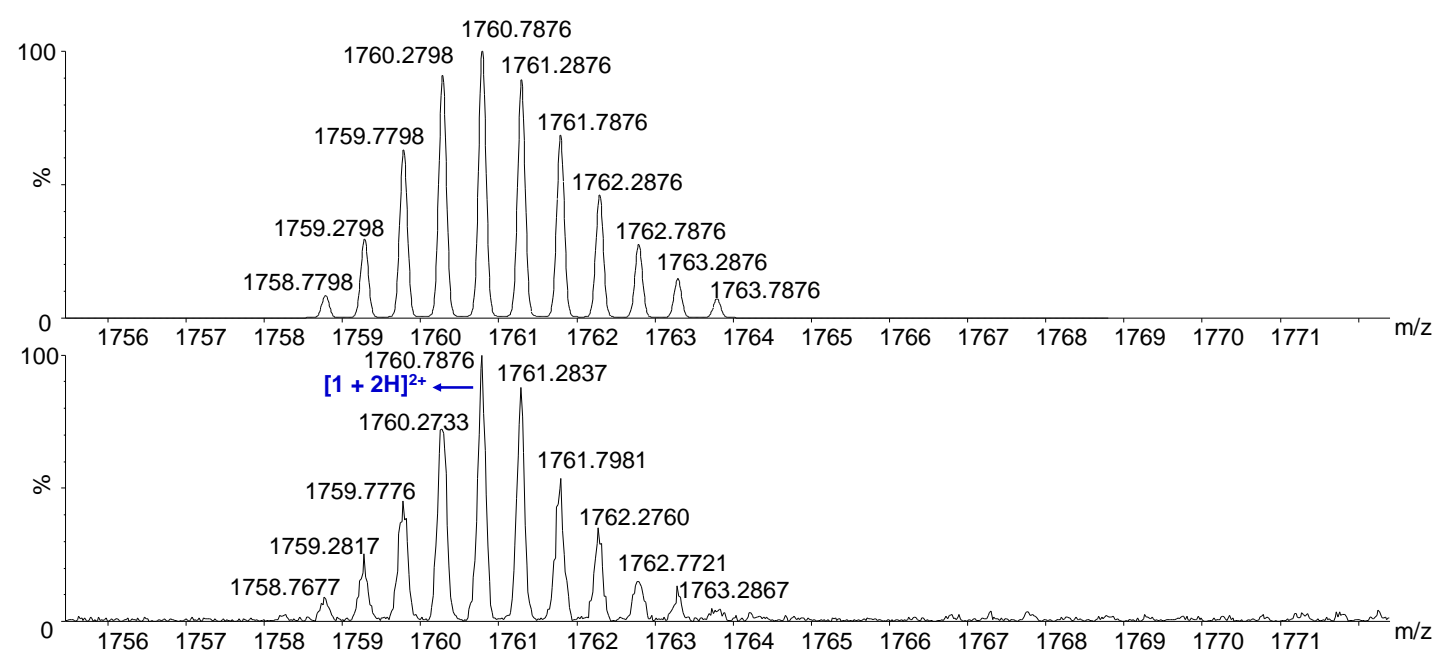

Figure S13: Theoretical (top) and experimental (bottom) ESI-TOF-MS spectra of the [c2]daisy chain rotaxane $\mathbf{1}$.

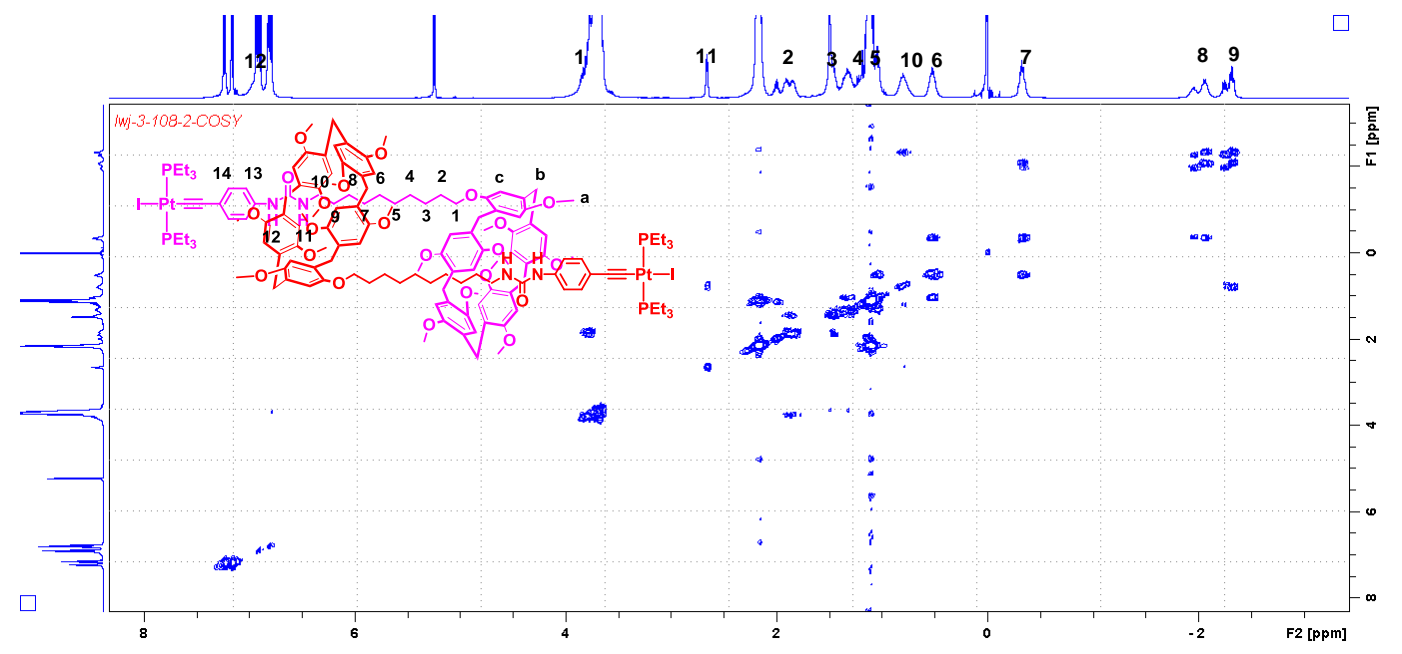

Figure S14: 2D ${ }^{1} \mathrm{H}-{ }^{1} \mathrm{H}$ COSY spectrum $\left(\mathrm{CD}_{2} \mathrm{Cl}_{2}, 298 \mathrm{~K}, 500 \mathrm{MHz}\right)$ of 2.

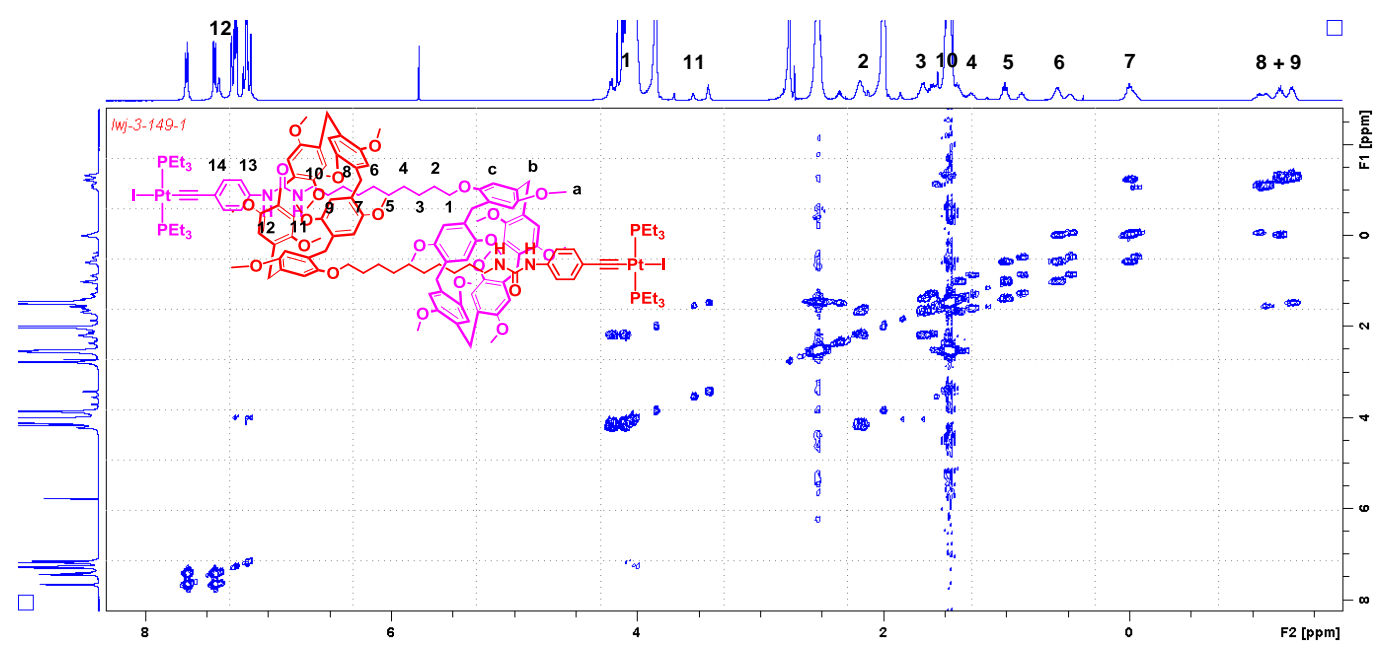

Figure S15: 2D ${ }^{1} \mathrm{H}-{ }^{1} \mathrm{H}$ COSY spectrum (THF- $\left.d_{8}, 298 \mathrm{~K}, 500 \mathrm{MHz}\right)$ of 2. 


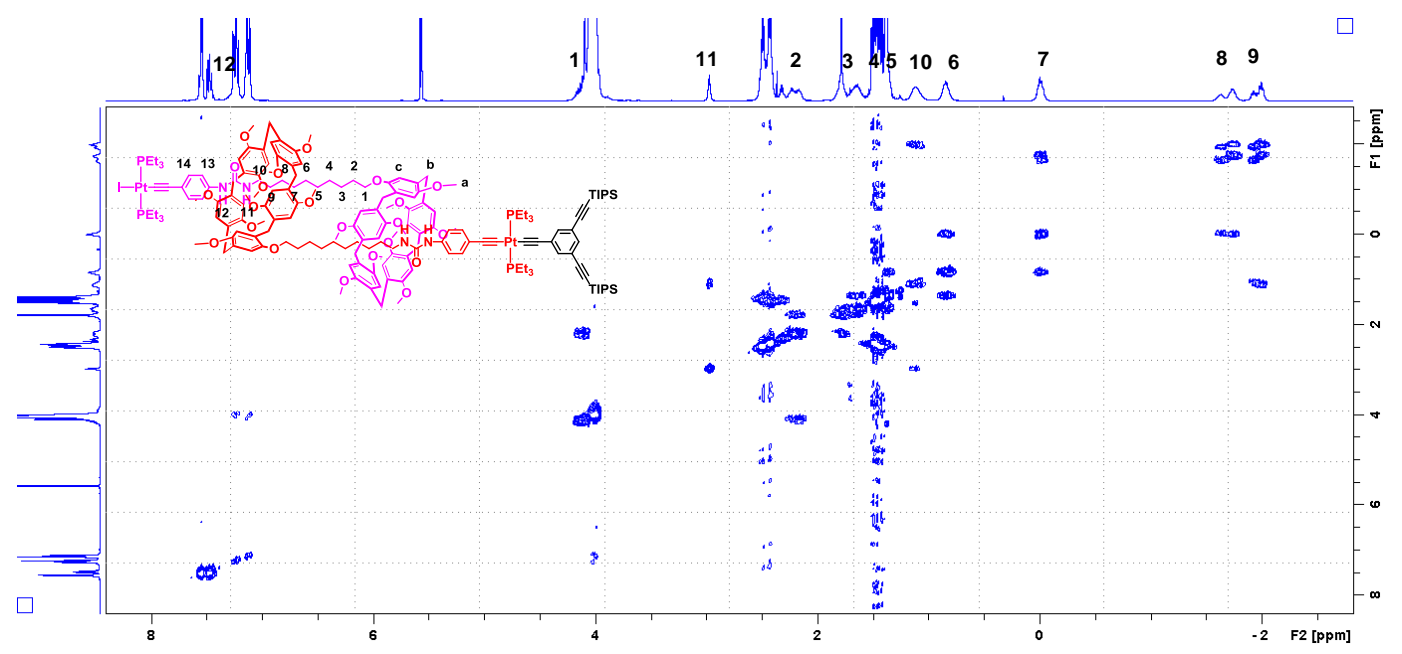

Figure S16: $2 \mathrm{D}{ }^{1} \mathrm{H}-{ }^{1} \mathrm{H}$ COSY spectrum $\left(\mathrm{CD}_{2} \mathrm{Cl}_{2}, 298 \mathrm{~K}, 500 \mathrm{MHz}\right)$ of $\mathbf{1}$.

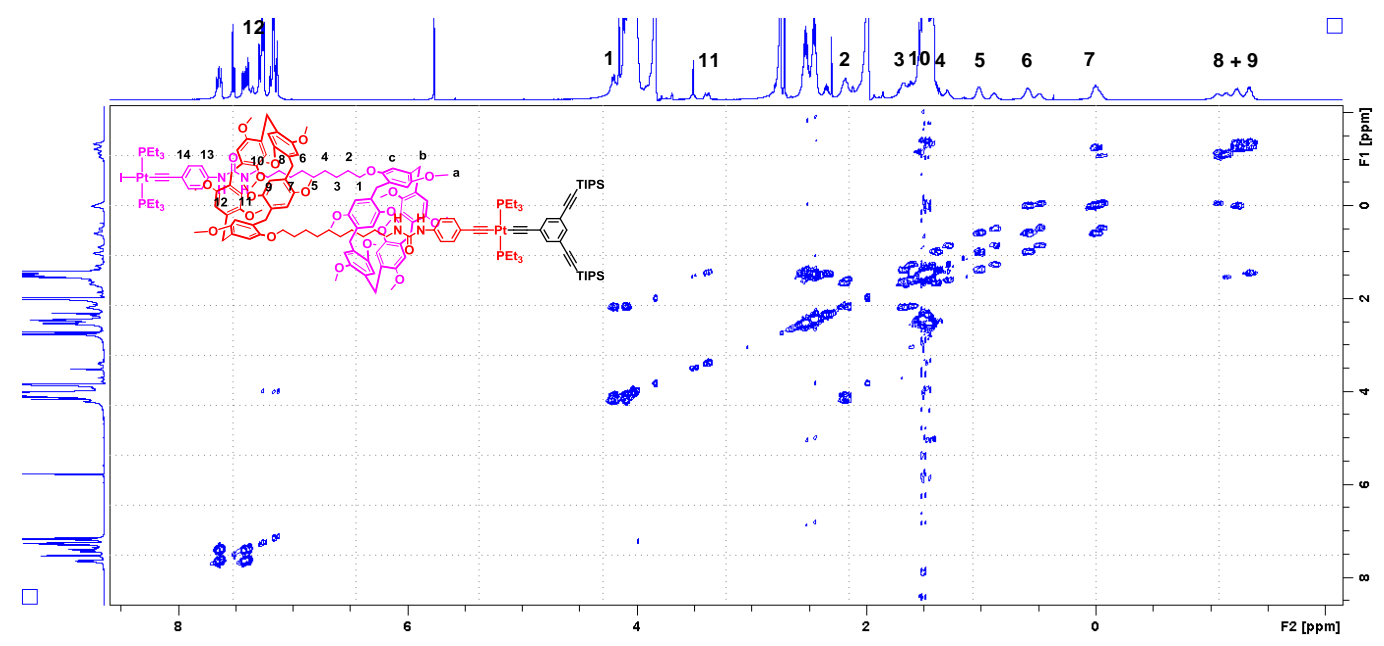

Figure S17: 2D ${ }^{1} \mathrm{H}-{ }^{1} \mathrm{H}$ COSY spectrum (THF- $d_{8}, 298 \mathrm{~K}, 500 \mathrm{MHz}$ ) of $\mathbf{1}$. 


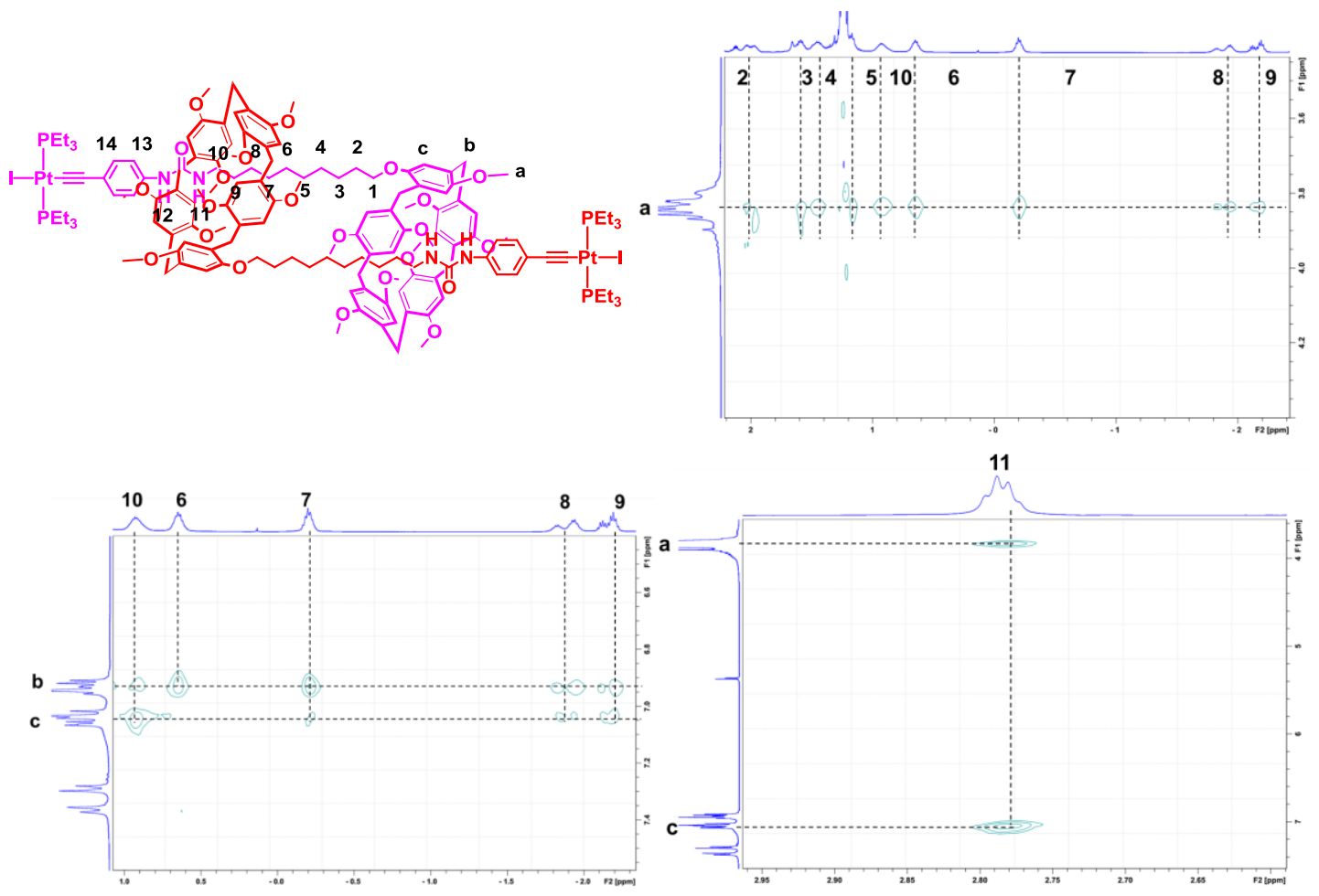

Figure S18: 2D ${ }^{1} \mathrm{H}-{ }^{1} \mathrm{H}$ ROESY spectra $\left(\mathrm{CD}_{2} \mathrm{Cl}_{2}, 298 \mathrm{~K}, 500 \mathrm{MHz}\right)$ of 2.
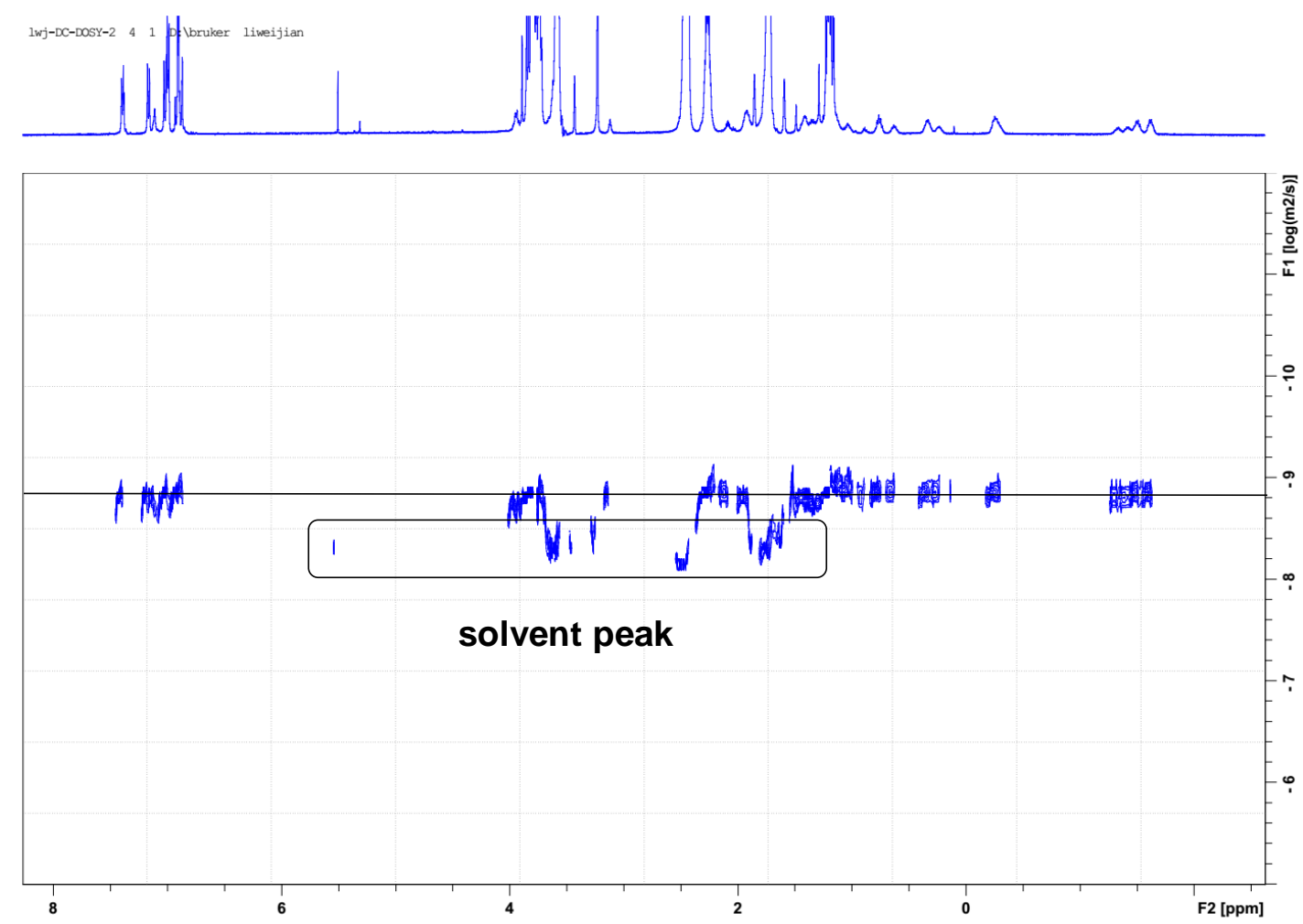

Figure S19: 2-D DOSY spectrum (THF-d8, $298 \mathrm{~K}, 500 \mathrm{MHz}$ ) of the [c2]daisy chain rotaxane 2. 


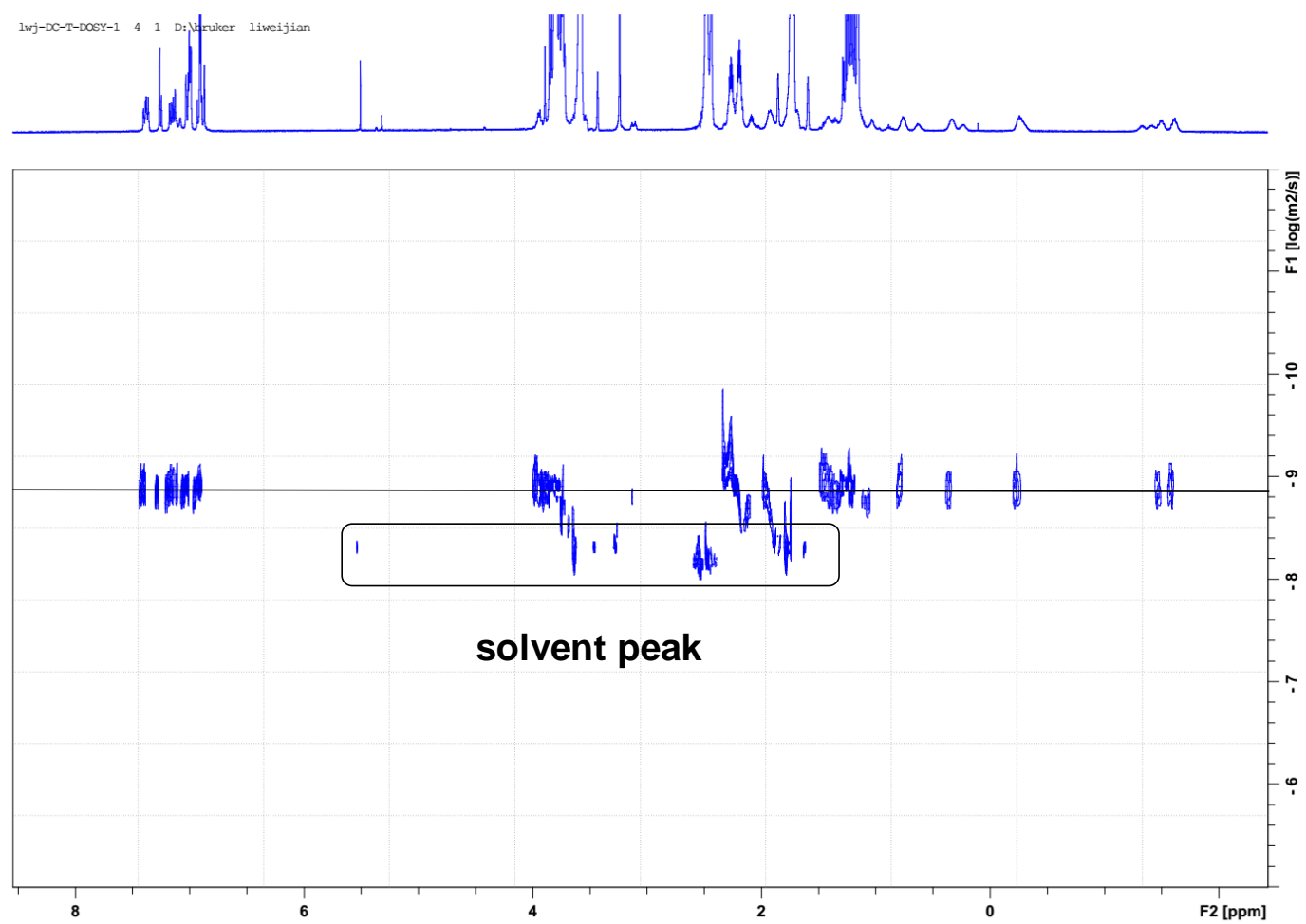

Figure S20: 2-D DOSY spectrum (THF- $d_{8}, 298 \mathrm{~K}, 500 \mathrm{MHz}$ ) of the [c2]daisy chain rotaxane 1 .

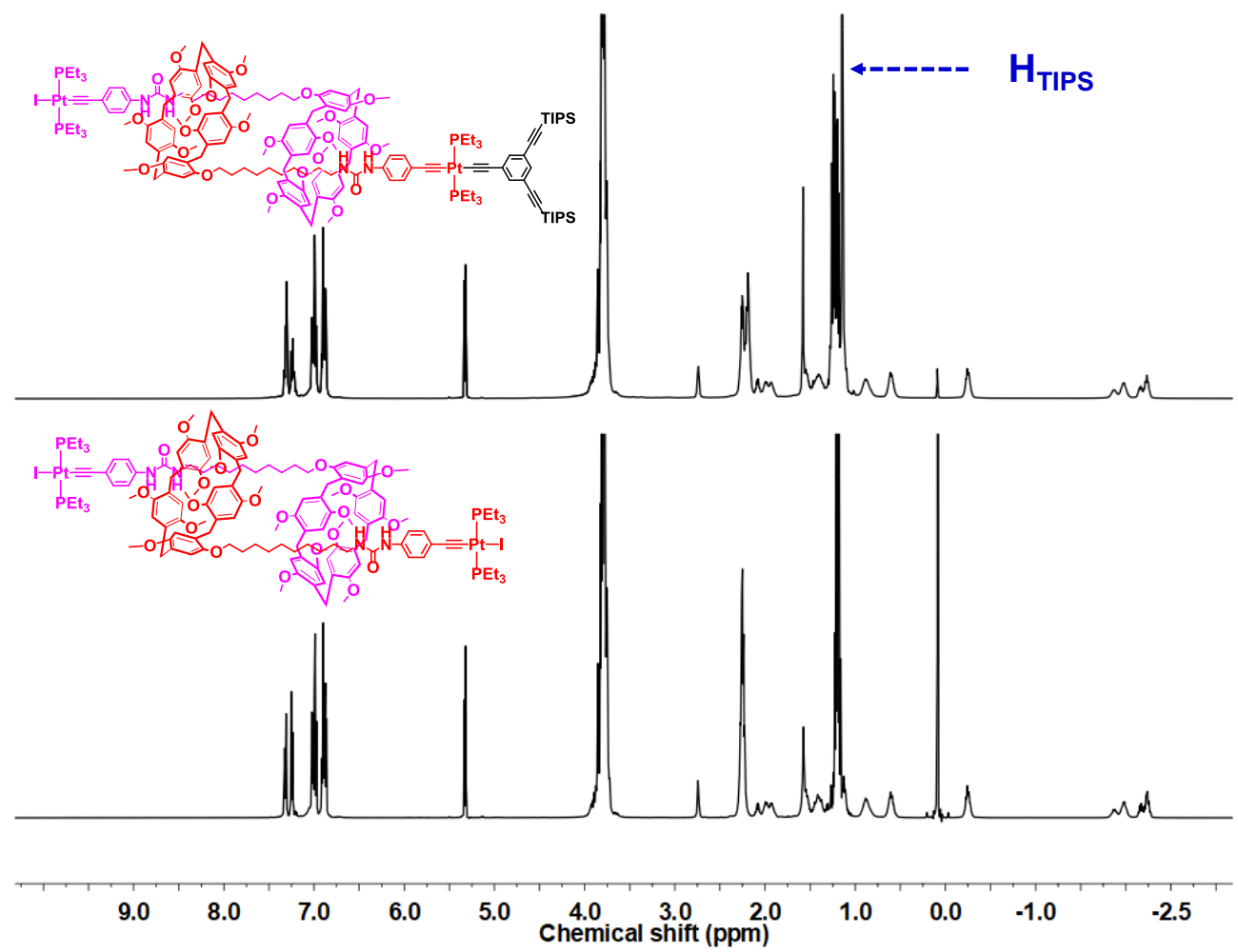

Figure S21: ${ }^{1} \mathrm{H}$ NMR spectra $\left(\mathrm{CD}_{2} \mathrm{Cl}_{2}, 298 \mathrm{~K}, 500 \mathrm{MHz}\right)$ of the [c2]daisy chain rotaxane 2 (bottom) and the [c2] daisy chain rotaxane $\mathbf{1}$ (top). 

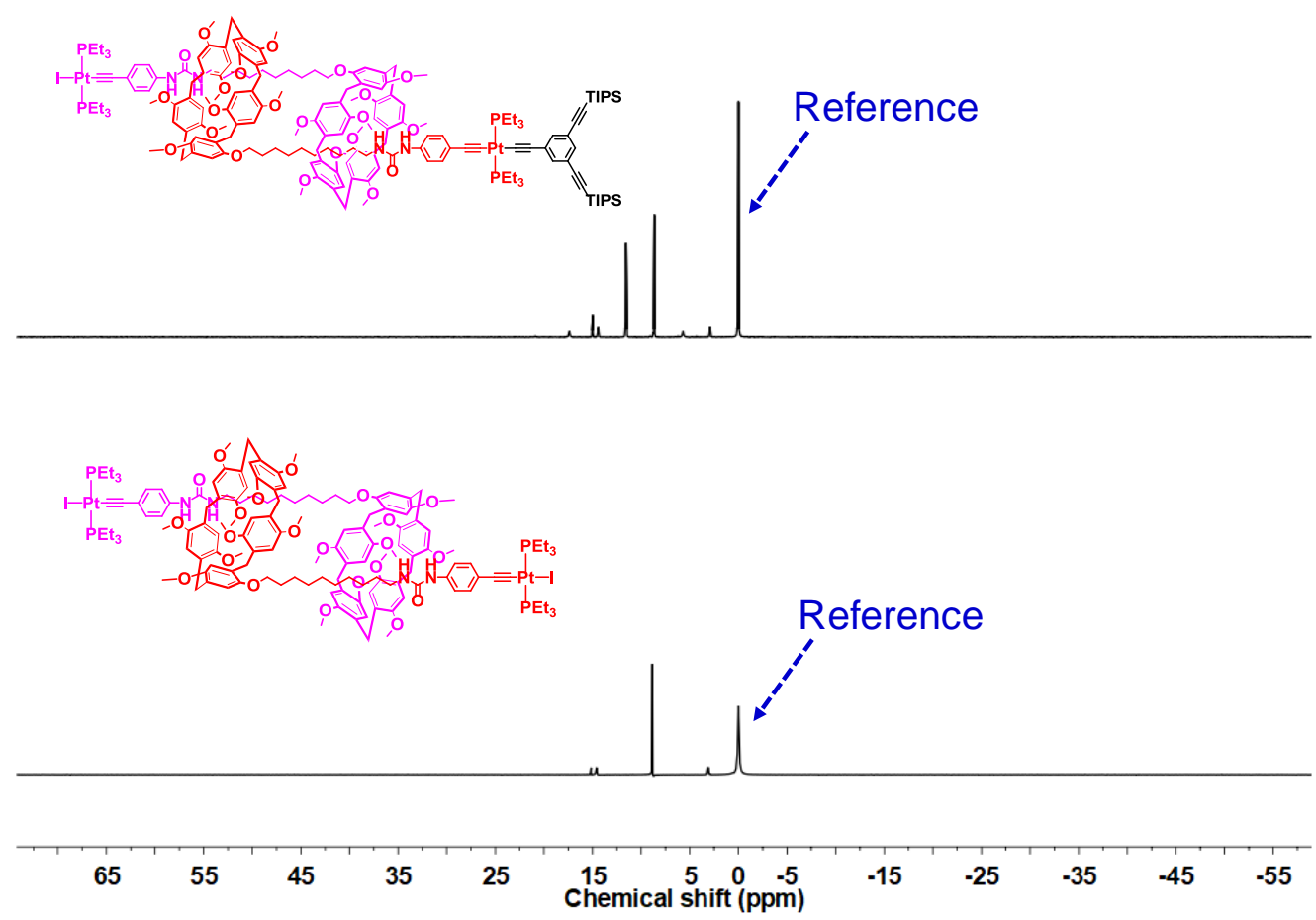

Figure S22: ${ }^{31} \mathrm{P}$ NMR spectra $\left(\mathrm{CD}_{2} \mathrm{Cl}_{2}, 298 \mathrm{~K}, 202 \mathrm{MHz}\right)$ of the [c2]daisy chain rotaxane $\mathbf{2}$ (bottom) and the [c2]daisy chain rotaxane $\mathbf{1}$ (top). 
Section 3. Synthesis and characterization of the daisy chain dendrimers

General procedure for the synthesis of daisy chain dendrimers DC-Gn: A mixture of multiyne complexes (for DC-G1, 1,3,5-tris(4-ethynylphenyl)benzene; for DC-G2, DC-G1-YNE; for DC-G3, DC-G2-YNE) and the [c2] daisy chain rotaxane 1 (for each terminal acetylene moiety, 1.1 equiv the [c2]daisy chain rotaxane $\mathbf{1}$ was added) in degassed dichloromethane/diethylamine (v/v, 1:1) was stirred for 12 hours at room temperature in the presence of a catalytic amount of $\mathrm{CuI}$. The solvent was evaporated and the residue was purified by column chromatography and gel permeation chromatography (GPC) to afford DC-Gn as pale-yellow solid.

General procedure for the synthesis of the deprotected daisy chain dendrimers DC-Gn-YNE: To a solution of DC-Gn in THF, a solution of tetrabutylammonium fluoride trihydrate in THF was added dropwise. The reaction mixture was stirred at room temperature for $4 \mathrm{~h}$. The obtained residue was washed by water, then dried over $\mathrm{Na}_{2} \mathrm{SO}_{4}$ and concentrated. The residue was further purified by column chromatography and gel permeation chromatography (GPC) to afford DC-Gn-YNE as pale-yellow solid.

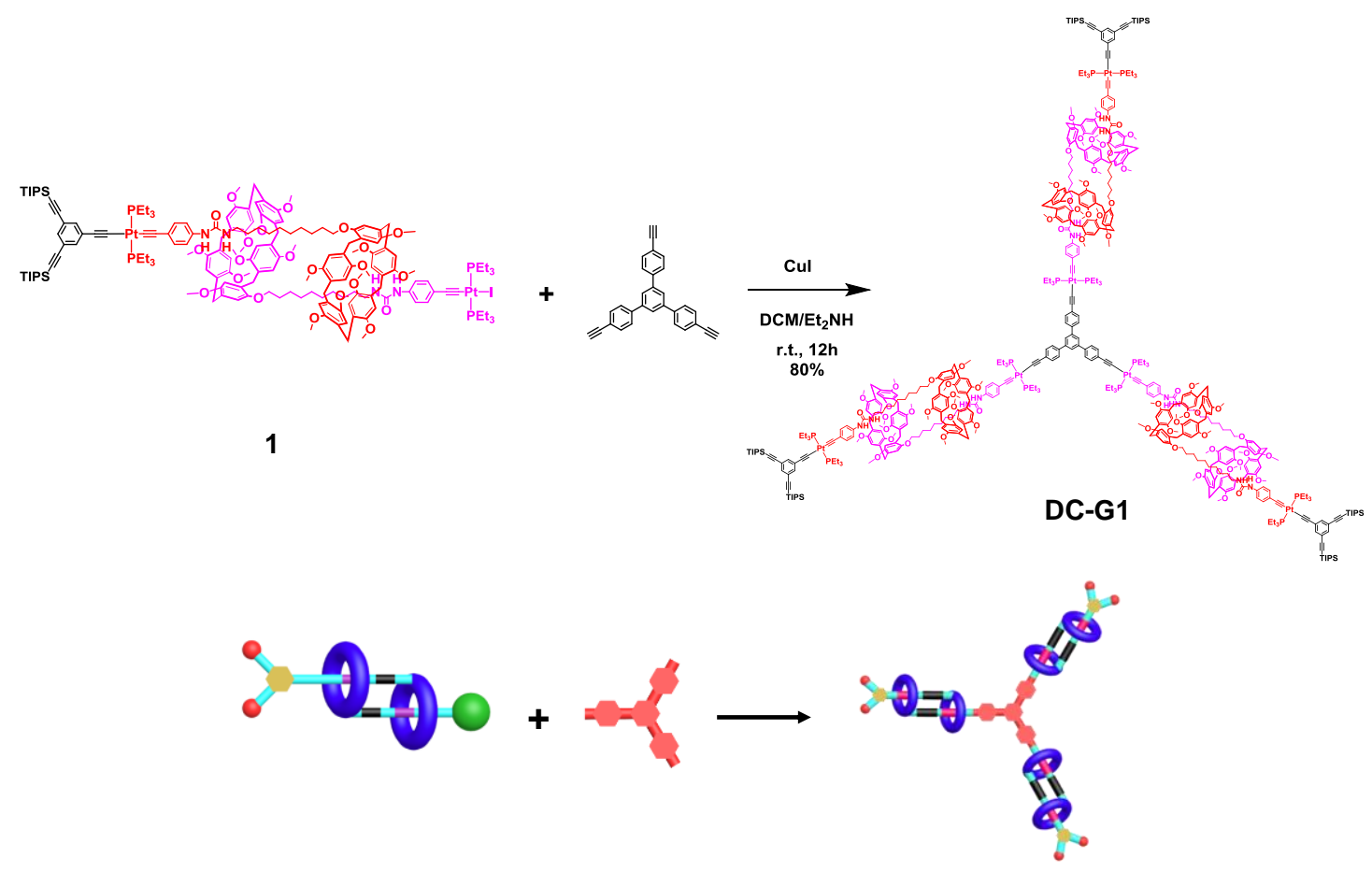

Scheme S2: Synthesis of the first-generation daisy chain dendrimer DC-G1. 
Synthesis of the first-generation daisy chain dendrimer DC-G1: A mixture of 1,3,5-tris(4-ethynylphenyl)benzene $(9.8 \mathrm{mg}, 0.026 \mathrm{mmol})$ and [c2]daisy chain monomer 1 (300 mg, $0.085 \mathrm{mmol}$ ) were added in a Schlenk flask, the Schlenk flask was then evacuated and back-filled with $\mathrm{N}_{2}$ three times. Next, degassed dichloromethane/diethylamine (v/v, 1:1) $(10 \mathrm{~mL})$ and a catalytic amount of $\mathrm{CuI}$ were added under an inert atmosphere. The reaction was stirred for 12 hours at room temperature. The solvent was evaporated and the residue was purified by column chromatography $\left(\mathrm{SiO}_{2} ; \mathrm{DCM} / \mathrm{EA}\right)$ and gel permeation chromatography (GPC) to yield a pale-yellow solid DC-G1 (218 mg, 80\%). ${ }^{1} \mathrm{H}$ NMR (500 MHz, $\left.\mathrm{CD}_{2} \mathrm{Cl}_{2}\right)$ : $\delta$ $7.78(\mathrm{~s}, 3 \mathrm{H}), 7.61-7.62(\mathrm{~m}, 6 \mathrm{H}), 7.40-7.42(\mathrm{~m}, 6 \mathrm{H}), 7.31-7.32(\mathrm{~m}, 21 \mathrm{H}), 7.24-7.25(\mathrm{~m}$, $12 \mathrm{H}), 6.98-7.03(\mathrm{~m}, 36 \mathrm{H}), 6.88-6.92(\mathrm{~m}, 30 \mathrm{H}), 3.77-3.86(\mathrm{~m}, 234 \mathrm{H}), 2.76(\mathrm{~m}, 6 \mathrm{H})$, 2.19-2.25 (m, 72H), 1.95-2.01 (m, 12H), $1.56(\mathrm{~m}, 12 \mathrm{H}), 1.42(\mathrm{~m}, 12 \mathrm{H}), 1.24-1.31(\mathrm{~m}$, $108 \mathrm{H}), 1.15(\mathrm{~s}, 138 \mathrm{H}), 0.89(\mathrm{~m}, 12 \mathrm{H}), 0.61(\mathrm{~m}, 12 \mathrm{H}),-0.23(\mathrm{~m}, 12 \mathrm{H}),-1.97-(-1.86)$ $(\mathrm{m}, 12 \mathrm{H}),-2.22-(-2.15)(\mathrm{m}, 12 \mathrm{H}) .{ }^{31} \mathrm{P}$ NMR $\left(202 \mathrm{MHz}, \mathrm{CD}_{2} \mathrm{Cl}_{2}\right): \delta 11.49,11.42 .{ }^{13} \mathrm{C}$ NMR (126 MHz, $\mathrm{CD}_{2} \mathrm{Cl}_{2}$ ): $\delta$ 154.06, 154.00, 150.81, 150.73, 150.64, 150.58, 150.52, $150.47,150.43,150.31,142.04,137.88,134.04,131.17,129.04,128.89,128.83$, $128.77,128.70,128.64,128.59,128.50,128.37,128.31,128.26,128.15,128.10$, $126.84,123.45,118.89,114.86,114.54,114.36,114.13,113.98,113.37,113.20$, 113.10, 112.90, 106.17, 90.97, 68.91, 68.65, 56.79, 56.58, 56.49, 56.44, 56.39, 56.27, $56.16,56.11,55.41,55.32,55.25,55.21,55.12,39.80,39.59,31.58,31.26,30.86$, $30.77,30.71,30.54,30.37,30.12,28.98,27.18,27.02,26.41,25.99,24.54,18.55$, $18.43,18.30,16.59,16.53,16.45,16.40,16.31,16.26,11.33,8.20,8.14$. LRMS (MALDI-TOF-MS): Calculated for [DC-G1 + Na] $]^{+}: \mathrm{m} / z=10570.0$; Found: $\mathrm{m} / z=$ 10568.9. Calculated for [DC-G1 $+\mathrm{Na}+\mathrm{H}]^{2+}: \mathrm{m} / z=5285.5$; Found: $m / z=5286.3$. 

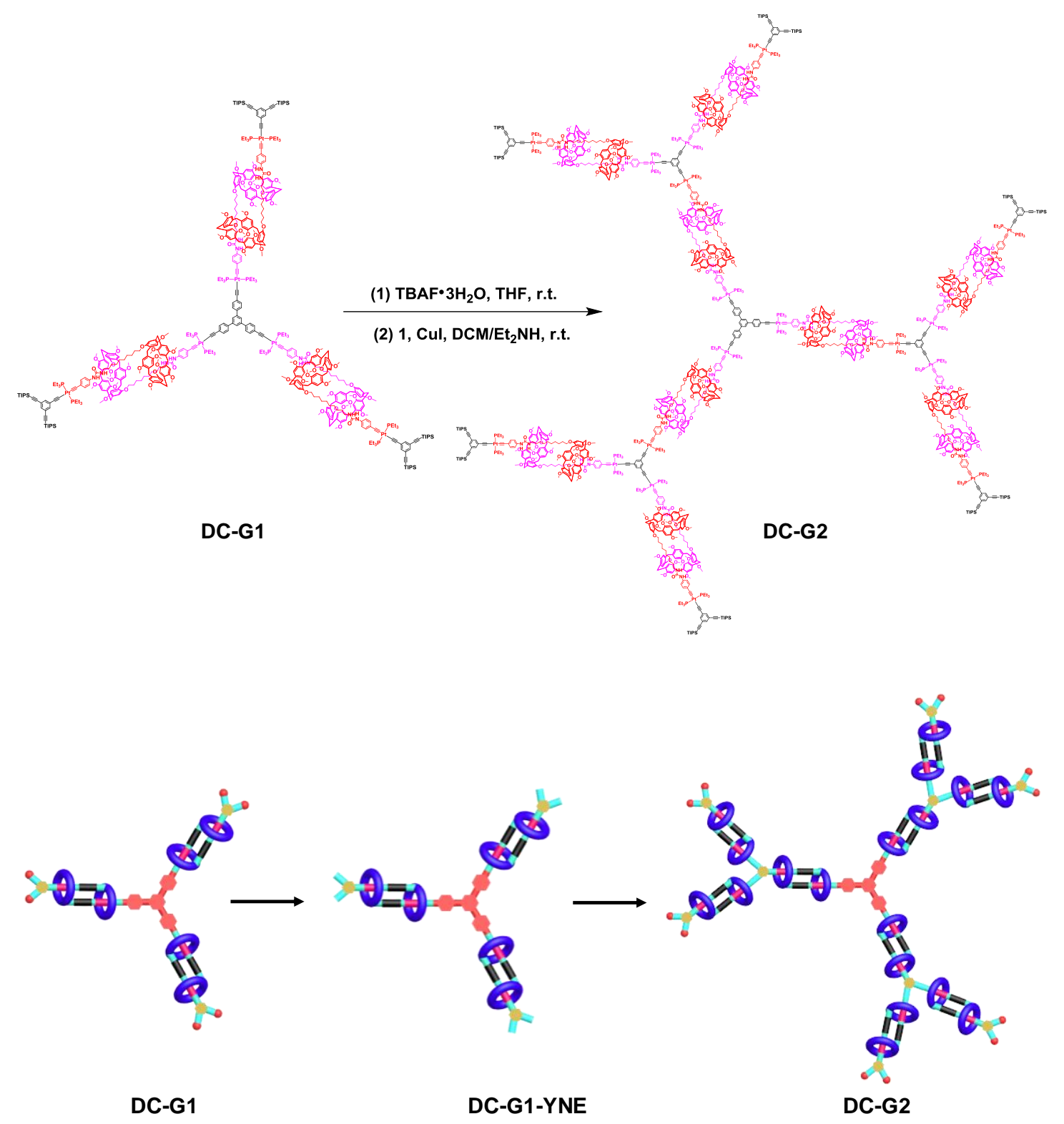

Scheme S3: Synthesis of the second-generation daisy chain dendrimer DC-G2.

Synthesis of the deprotected first-generation daisy chain dendrimer DC-G1-YNE: A solution of DC-G1 (107 mg, $0.010 \mathrm{mmol})$ in THF (30 mL) and then a solution of tetrabutylammonium fluoride trihydrate $(40 \mathrm{mg}, 0.127 \mathrm{mmol})$ in THF $(30 \mathrm{~mL})$ was added dropwise into the reaction flask. The reaction mixture was stirred at room temperature for $4 \mathrm{~h}$. The obtained residue was washed by water, then dried with $\mathrm{Na}_{2} \mathrm{SO}_{4}$ and concentrated. The residue was further purified by column chromatography $\left(\mathrm{SiO}_{2}\right.$; DCM/EA) to afford a pale-yellow solid $(74 \mathrm{mg}, 76 \%) .{ }^{1} \mathrm{H}$ NMR (500 MHz, $\left.\mathrm{CD}_{2} \mathrm{Cl}_{2}\right): \delta 7.78(\mathrm{~s}, 3 \mathrm{H}), 7.61-7.62(\mathrm{~m}, 6 \mathrm{H}), 7.39-7.41(\mathrm{~m}, 6 \mathrm{H}), 7.36$ (s, 9H), 7.30-7.31 (m, 12H), 7.22-7.25 (m, 12H), 6.98-7.03 (m, 36H), 6.87-6.92 (m, $30 \mathrm{H}), 3.87-3.86(\mathrm{~m}, 234 \mathrm{H}), 3.14(\mathrm{~s}, 6 \mathrm{H}), 2.74(\mathrm{~m}, 6 \mathrm{H}), 2.18-2.26(\mathrm{~m}, 72 \mathrm{H}), 1.93-2.01$ $(\mathrm{m}, 12 \mathrm{H}), 1.55(\mathrm{~m}, 12 \mathrm{H}), 1.41(\mathrm{~m}, 12 \mathrm{H}), 1.21-1.30(\mathrm{~m}, 108 \mathrm{H}), 1.13(\mathrm{~m}, 12 \mathrm{H}), 0.89$ (m, 
12H), $0.61(\mathrm{~m}, 12 \mathrm{H}),-0.24(\mathrm{~m}, 12 \mathrm{H}),-1.97-(-1.87)(\mathrm{m}, 12 \mathrm{H}),-2.23-(-2.16)(\mathrm{m}, 12 \mathrm{H})$. ${ }^{31} \mathrm{P}$ NMR $\left.\left(202 \mathrm{MHz}, \mathrm{CD}_{2} \mathrm{Cl}_{2}\right): \delta 11.88,11.78 .{ }^{13} \mathrm{C} \mathrm{NMR} \mathrm{(126} \mathrm{MHz,} \mathrm{CD}_{2} \mathrm{Cl}_{2}\right): \delta$ $154.07,154.00,150.80,150.72,150.63,150.57,150.51,150.46,150.29,142.04$, $137.90,137.52,134.54,131.69,131.21,131.15,129.66,129.02,128.87,128.81$, $128.76,128.68,128.63,128.58,128.49,128.36,128.30,128.25,128.14,128.10$, $126.83,122.22,118.87,114.83,114.52,114.40,114.11,113.98,113.36,113.10$, $112.89,109.35,107.19,82.49,77.43,68.91,68.66,59.11,56.78,56.56,56.47,56.43$, $56.37,56.26,56.15,56.10,55.41,55.32,55.25,55.22,55.11,39.80,39.59,31.56$, $31.24,30.85,30.75,30.71,30.52,30.36,30.10,29.88,29.69,28.98,27.17,27.00$, $26.45,26.02$, 24.56, 24.06, 19.76, 16.58, 16.53, 16.44, 16.39, 16.30, 16.25, 13.42, 8.19, 8.14. LRMS (MALDI-TOF-MS): Calculated for [DC-G1-YNE $+\mathrm{K}]^{+}: m / z=$ 9649.2; Found: $m / z=9696.6$. Calculated for [DC-G1-YNE $+2 \mathrm{Na}]^{2+}: \mathrm{m} / z=4828.1$; Found: $m / z=4828.3$.

Synthesis of the second-generation daisy chain dendrimer DC-G2: A mixture of DC-G1-YNE (74 mg, $0.008 \mathrm{mmol})$ and [c2]daisy chain monomer 1 (179 mg, 0.501 mmol) were added in a Schlenk flask, the Schlenk flask was then evacuated and back-filled with $\mathrm{N}_{2}$ three times. Next, degassed dichloromethane/diethylamine (v/v, 1:1) $(20 \mathrm{~mL})$ and a catalytic amount of CuI were added under an inert atmosphere. The reaction was stirred for 12 hours at room temperature. The solvent was evaporated and the residue was purified by column chromatography $\left(\mathrm{SiO}_{2} ; \mathrm{DCM} / \mathrm{EA}\right)$ and gel permeation chromatography (GPC) to yield a pale-yellow solid DC-G2 (194 mg, 84\%). ${ }^{1} \mathrm{H}$ NMR (500 MHz, $\mathrm{CD}_{2} \mathrm{Cl}_{2}$ ): $\delta 7.77$ (s), 7.60 (m), 7.39 (m), 7.30-7.31 (m), 7.21-7.24 (m), 6.97-7.03 (m), 6.87-6.91 (m), 3.78-3.85 (m), $2.73(\mathrm{~m}), 2.17-2.24(\mathrm{~m})$, 1.93-1.99 (m), $1.54(\mathrm{~m}), 1.40(\mathrm{~m}), 1.22-1.29(\mathrm{~m}), 1.14(\mathrm{~s}), 0.88(\mathrm{~m}), 0.60(\mathrm{~m}),-0.25$ (m), -1.97- (-1.87) (m), -2.23- (-2.16) (m). ${ }^{31} \mathrm{P}$ NMR (202 MHz, $\left.\mathrm{CD}_{2} \mathrm{Cl}_{2}\right): \delta 11.54$, 11.37. ${ }^{13} \mathrm{C}$ NMR (126 MHz, $\left.\mathrm{CD}_{2} \mathrm{Cl}_{2}\right): \delta 154.09,150.82,150.74,150.64,150.58$, $150.52,150.47,150.31,137.85,134.05,131.16,129.36,129.04,128.89,128.83$, $128.77,128.70,128.64,128.59,128.50,128.37,128.31,128.26,128.15,128.11$, $126.84,123.45,118.92,114.86,114.54,114.36,114.13,113.98,113.37,113.21$, $113.11,112.88,106.18,90.97,68.92,68.66,56.79,56.58,56.49,56.45,56.40,56.28$, $56.17,56.12,55.42,55.32,55.25,55.22,55.12,39.81,39.60,31.58,31.26,30.86$, $30.77,30.71,30.54,30.37,30.11,28.99,27.18,27.02,25.99,24.54,18.43,16.56$, $16.53,16.42,16.39,16.28,16.25,11.33,8.19,8.14$. LRMS (MALDI-TOF-MS): Calculated for $M_{\mathrm{r}}=29970.4 \mathrm{Da}$; Found: $m / z=30684.2$. 

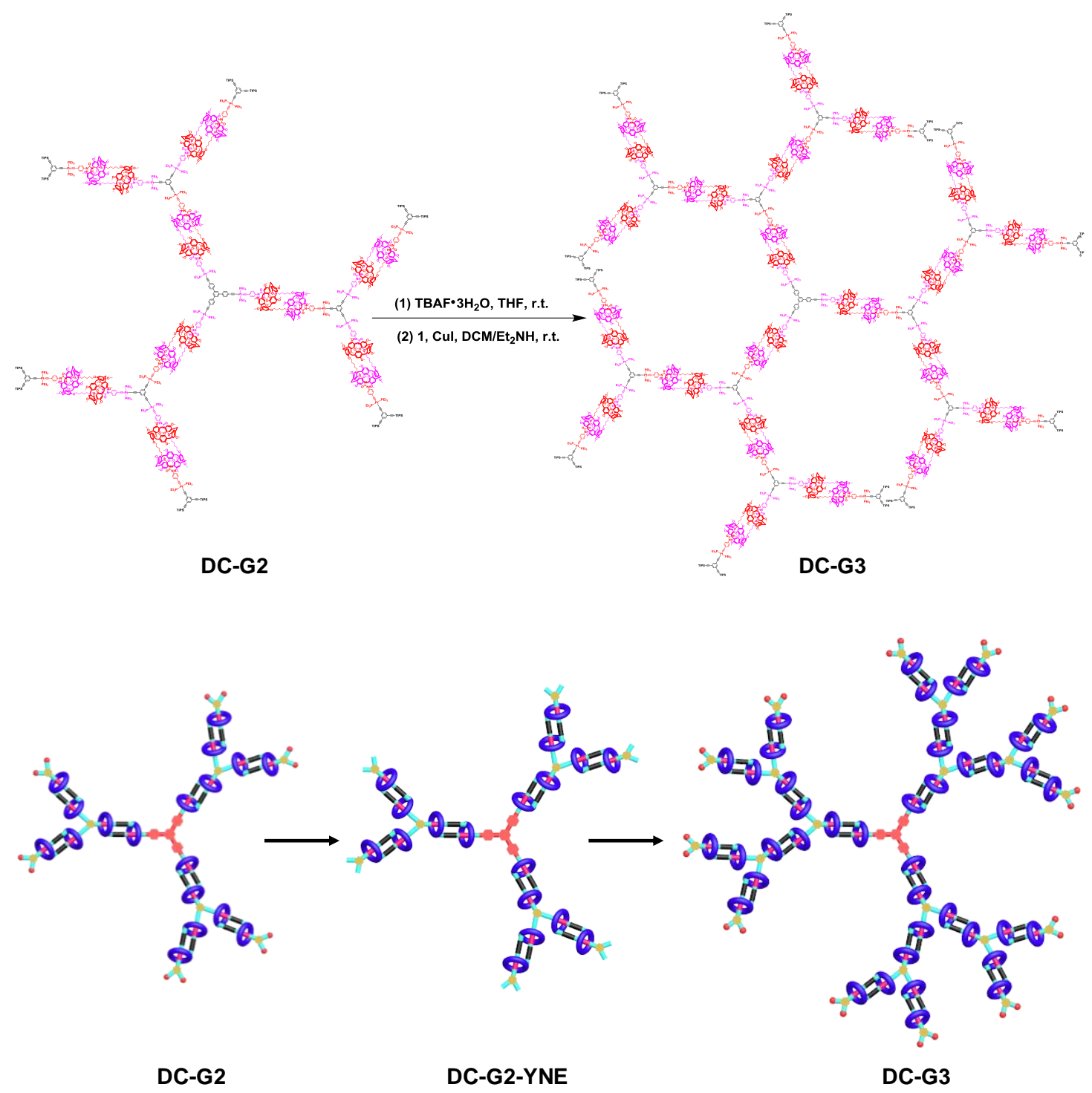

Scheme S4: Synthesis of the third-generation daisy chain dendrimer DC-G3.

Synthesis of the deprotected second-generation daisy chain dendrimer DC-G2-YNE: A solution of DC-G2 (100 mg, $0.0033 \mathrm{mmol})$ in THF (30.0 mL) and then a solution of tetrabutylammonium fluoride trihydrate $(25 \mathrm{mg}, 0.079 \mathrm{mmol})$ in THF (30 mL) was added dropwise into the reaction flask. The reaction mixture was stirred at room temperature for $4 \mathrm{~h}$. The obtained residue was washed by water, then dried with $\mathrm{Na}_{2} \mathrm{SO}_{4}$ and concentrated. The residue was further purified by column chromatography $\left(\mathrm{SiO}_{2}\right.$; DCM/EA) to afford a pale-yellow solid $(76 \mathrm{mg}, 81 \%) .{ }^{1} \mathrm{H}$ NMR (500 MHz, $\mathrm{CD}_{2} \mathrm{Cl}_{2}$ ): $\delta 7.78(\mathrm{~s}), 7.61(\mathrm{~m}), 7.39(\mathrm{~m}, 6 \mathrm{H}), 7.36(\mathrm{~s}), 7.30-7.32(\mathrm{~m})$, 7.22-7.25 (m), 6.98-7.03 (m), 6.87-6.92 (m), 3.76-3.86 (m), $3.14(\mathrm{~s}), 2.74(\mathrm{~m})$, 2.16-2.26 (m), 1.93-2.01 (m), $1.56(\mathrm{~m}), 1.42(\mathrm{~m}), 1.23-1.31(\mathrm{~m}), 1.13(\mathrm{~m}), 0.88(\mathrm{~m})$, $0.61(\mathrm{~m}),-0.25(\mathrm{~m}),-1.98-(-1.87)(\mathrm{m}),-2.23-(-2.16)(\mathrm{m}) .{ }^{31} \mathrm{P}$ NMR $(202 \mathrm{MHz}$, $\left.\mathrm{CD}_{2} \mathrm{Cl}_{2}\right): \delta 11.88,11.70 .{ }^{13} \mathrm{C} \mathrm{NMR}\left(126 \mathrm{MHz}, \mathrm{CD}_{2} \mathrm{Cl}_{2}\right): \delta 154.09,150.81,150.73$, $150.63,150.58,150.46,150.31,137.88,134.54,131.70,131.16,129.67,129.03$, 
$128.76,128.69,128.64,128.58,128.49,128.37,128.30,128.26,128.14,128.10$, $126.84,122.22,118.90,114.85,114.52,114.42,114.11,113.98,113.37,113.10$, 107.21, 82.49, 77.44, 68.92, 68.66, 59.32, 56.78, 56.57, 56.48, 56.44, 56.27, 56.16, $55.42,55.32,55.25,55.12,39.80,39.60,31.56,31.25,30.86,30.71,30.52,30.36$, $30.10,29.88,29.70,28.99,27.17,27.01,26.45,26.01,24.55,24.18,19.85,16.55$, 16.41, 16.27, 13.50, 8.19, 8.15. LRMS (MALDI-TOF-MS): Calculated for $[\mathrm{M}]^{+}: \mathrm{m} / \mathrm{z}$ $=28074.4$; Found: $m / z=28074.0$.

Synthesis of the third-generation daisy chain dendrimer DC-G3: A mixture of DC-G2-YNE (76 mg, $0.0027 \mathrm{mmol}$ ) and [c2]daisy chain monomer 1 (126 mg, 0.036 mmol) were added in a Schlenk flask, the Schlenk flask was then evacuated and back-filled with $\mathrm{N}_{2}$ three times. Next, degassed dichloromethane/diethylamine (v/v, 1:1) $(20 \mathrm{~mL})$ and a catalytic amount of $\mathrm{CuI}$ were added under an inert atmosphere. The reaction was stirred for 12 hours at room temperature. The solvent was evaporated and the residue was purified by column chromatography $\left(\mathrm{SiO}_{2} ; \mathrm{DCM} / \mathrm{EA}\right)$ and gel permeation chromatography (GPC) to yield a pale-yellow solid DC-G3 (121 $\mathrm{mg}, 65 \%) .{ }^{1} \mathrm{H}$ NMR (500 MHz, $\mathrm{CD}_{2} \mathrm{Cl}_{2}$ ): $\delta 7.77(\mathrm{~s}), 7.61(\mathrm{~m}), 7.40(\mathrm{~m}), 7.31(\mathrm{~m})$, $7.24(\mathrm{~m}), 7.00(\mathrm{~m}), 6.88-6.91(\mathrm{~m}), 3.80(\mathrm{~m}), 2.75(\mathrm{~m}), 2.22(\mathrm{~m}), 1.94-2.00(\mathrm{~m}), 1.55$ (m), $1.41(\mathrm{~m}), 1.25-1.27(\mathrm{~m}), 1.15(\mathrm{~s}), 0.89(\mathrm{~m}), 0.61(\mathrm{~m}),-0.24(\mathrm{~m}),-1.97-(-1.87)$ (m), -2.22- (-2.16) (m). ${ }^{31} \mathrm{P}$ NMR (202 MHz, $\left.\mathrm{CD}_{2} \mathrm{Cl}_{2}\right): \delta 11.87,11.70 .{ }^{13} \mathrm{C}$ NMR $(126$ $\left.\mathrm{MHz}, \mathrm{CD}_{2} \mathrm{Cl}_{2}\right): \delta 154.06,150.81,150.72,150.63,150.57,150.46,150.31,137.82$, $134.05,131.14,129.03,128.88,128.82,128.76,128.69,128.63,128.58,128.49$, $128.36,128.30,128.25,128.14,128.10,123.44,118.89,114.42,114.11,113.98$, 113.36, 113.10, 106.17, 90.95, 68.66, 56.78, 56.56, 56.48, 56.43, 56.38, 56.26, 56.15, $55.41,55.32,55.25,55.21,55.12,42.80,39.80,39.59,31.94,31.57,31.26,30.86$, $30.77,30.71,30.53,30.37,30.07,29.90,29.70,29.37,28.99,27.18,27.01,26.43$, $26.00,24.55,22.71,18.43,16.55,16.53,16.41,16.39,16.27,11.33,8.19,8.13$. 


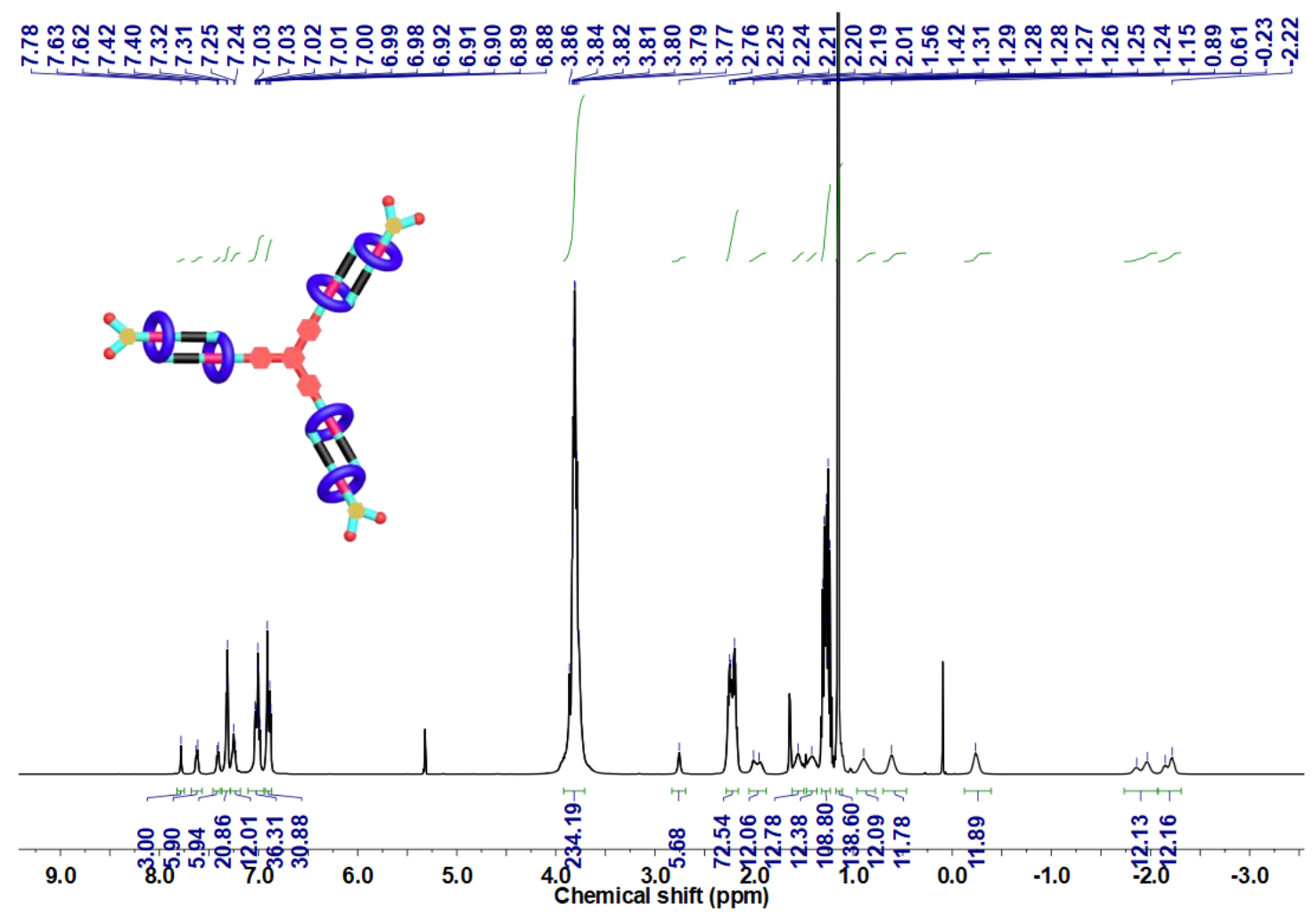

Figure S23: ${ }^{1} \mathrm{H}$ NMR spectrum $\left(\mathrm{CD}_{2} \mathrm{Cl}_{2}, 298 \mathrm{~K}, 500 \mathrm{MHz}\right)$ of the daisy chain dendrimer DC-G1.
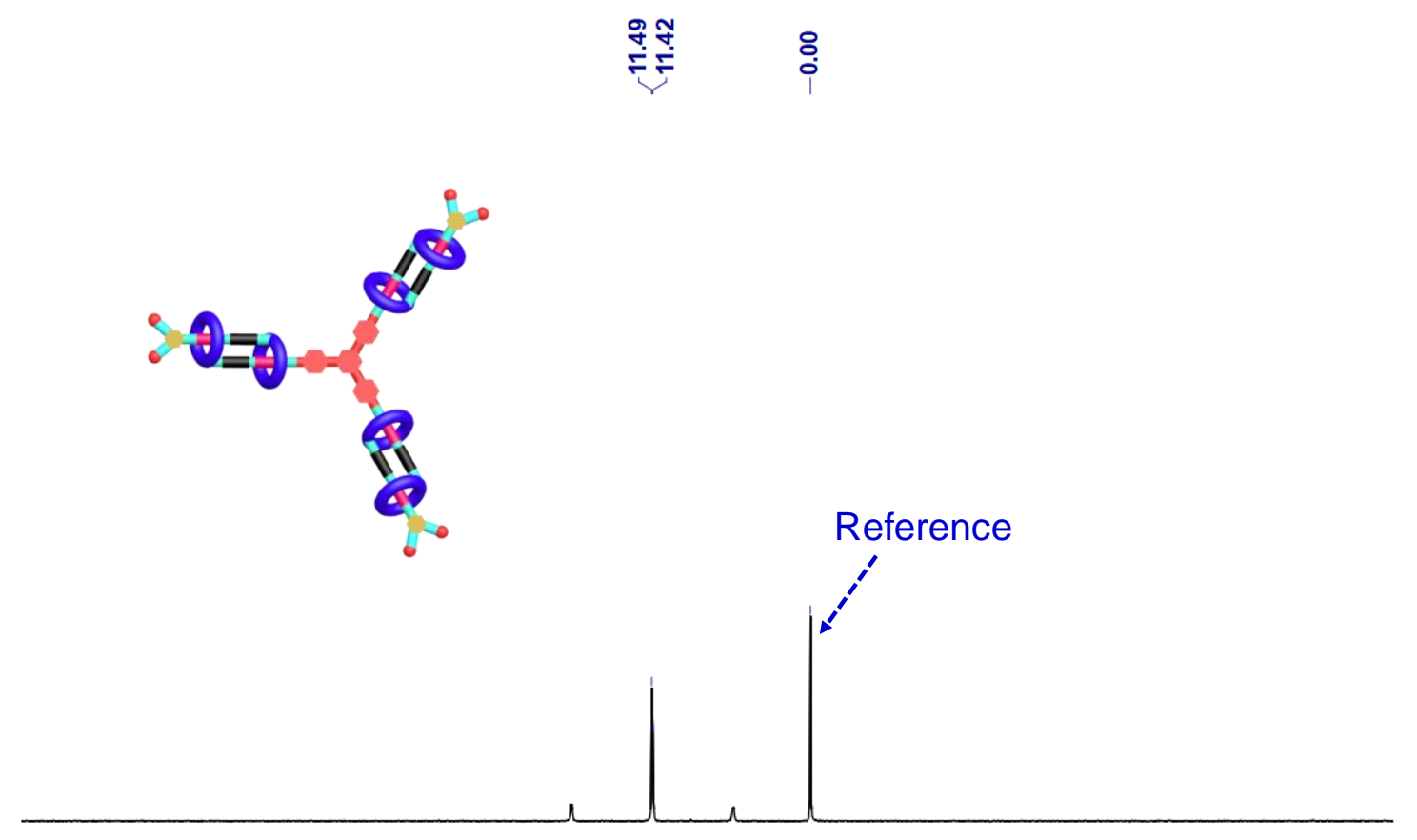

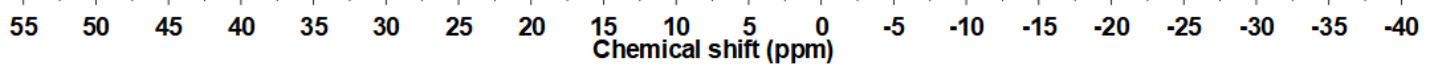

Figure S24: ${ }^{31} \mathrm{P}$ NMR spectrum $\left(\mathrm{CD}_{2} \mathrm{Cl}_{2}, 298 \mathrm{~K}, 202 \mathrm{MHz}\right)$ of the daisy chain dendrimer DC-G1. 


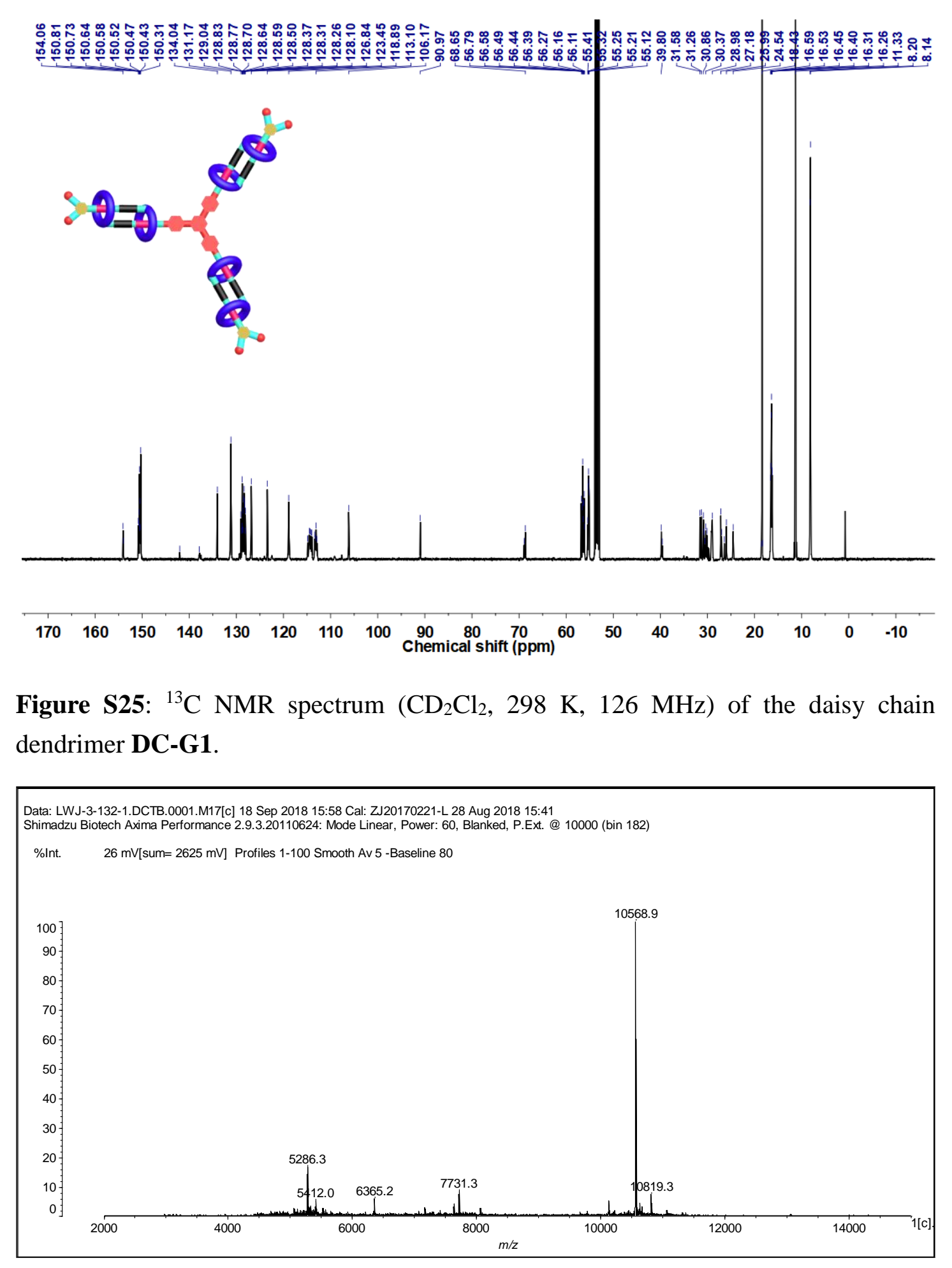

Figure S26: MALDI-TOF-MS spectrum of the daisy chain dendrimer DC-G1. 


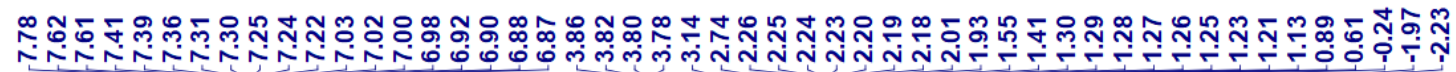

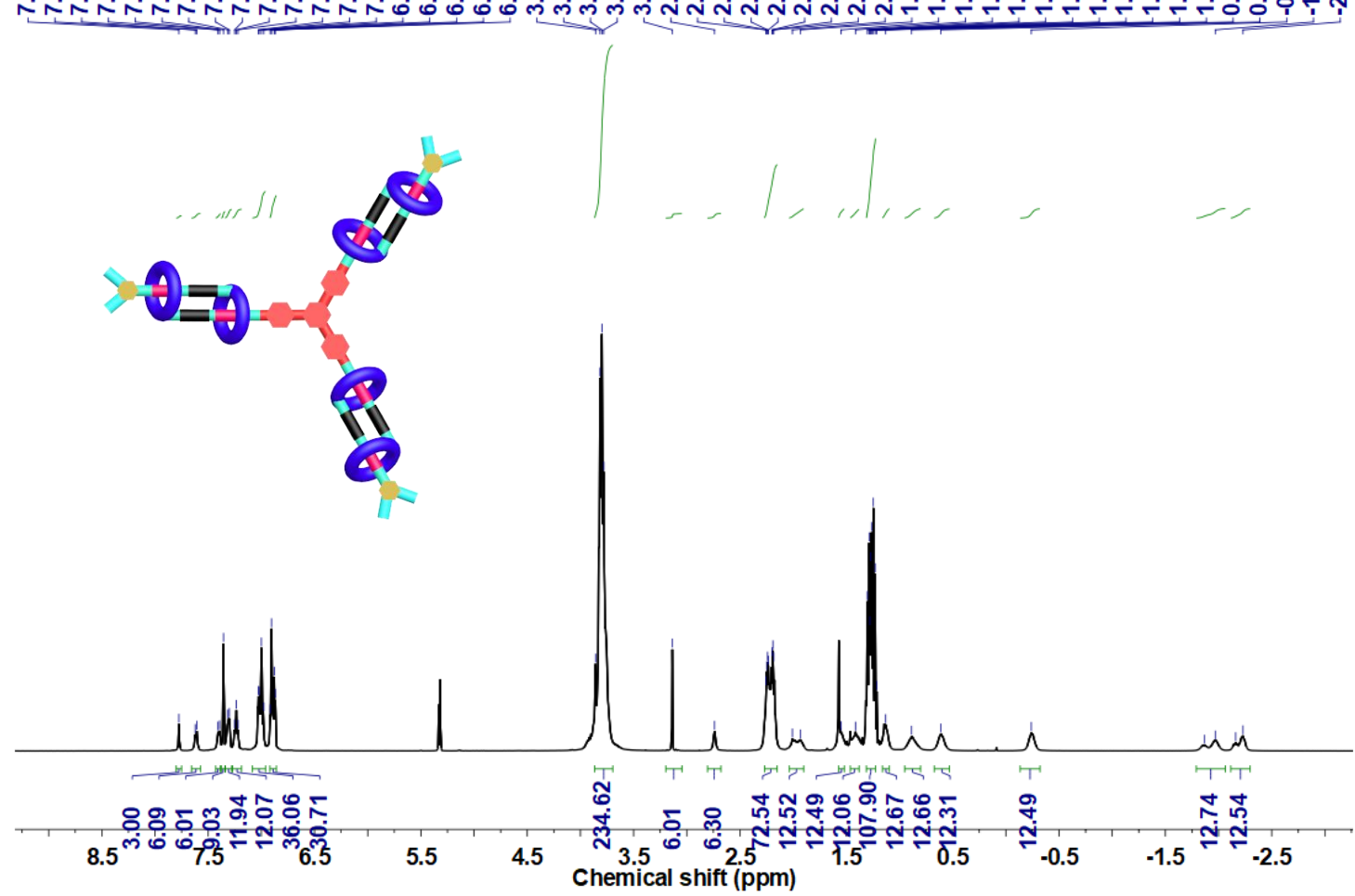

Figure S27: ${ }^{1} \mathrm{H}$ NMR spectrum $\left(\mathrm{CD}_{2} \mathrm{Cl}_{2}, 298 \mathrm{~K}, 500 \mathrm{MHz}\right)$ of the daisy chain dendrimer DC-G1-YNE.
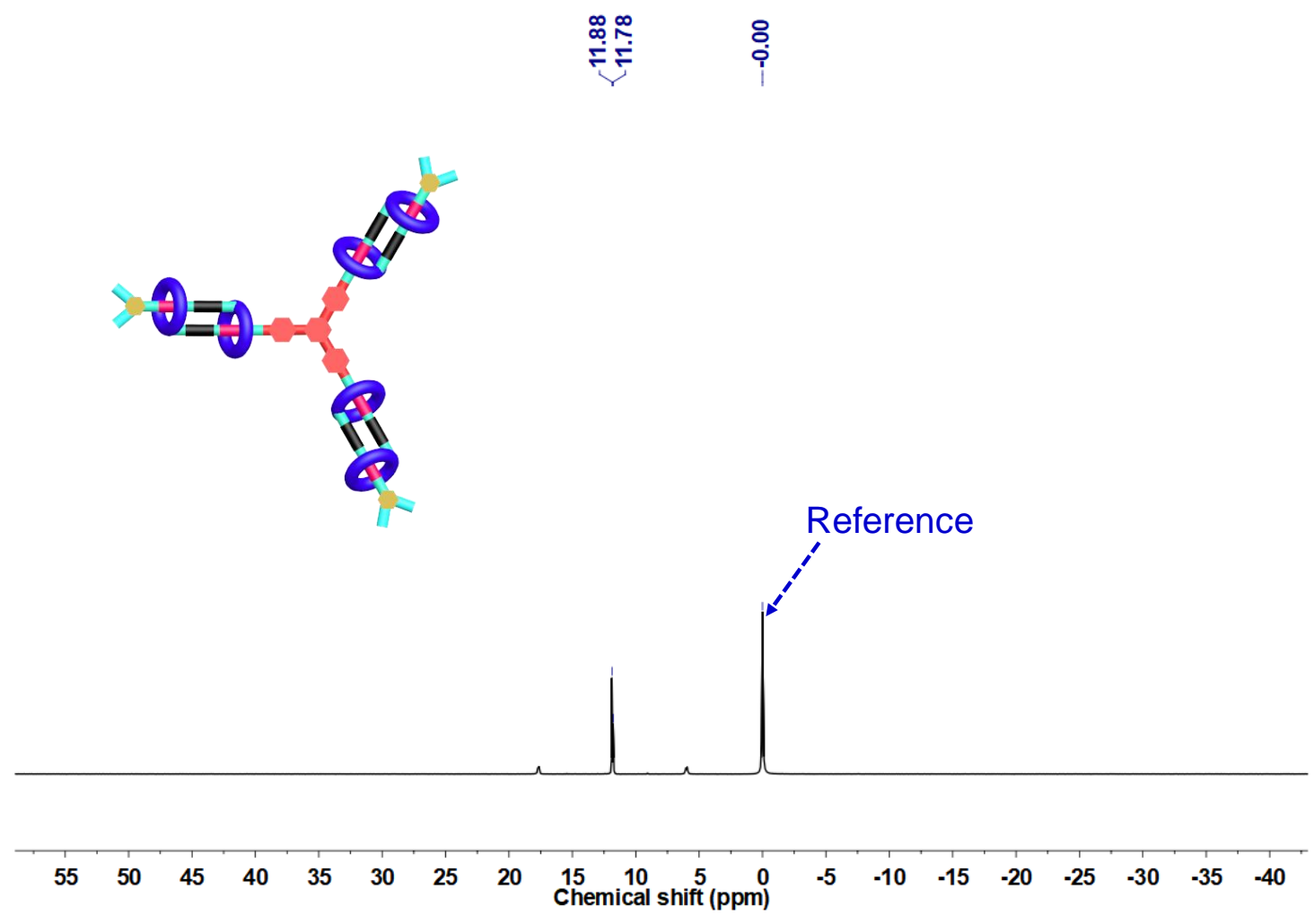

Figure S28: ${ }^{31} \mathrm{P}$ NMR spectrum $\left(\mathrm{CD}_{2} \mathrm{Cl}_{2}, 298 \mathrm{~K}, 202 \mathrm{MHz}\right)$ of the daisy chain dendrimer DC-G1-YNE. 


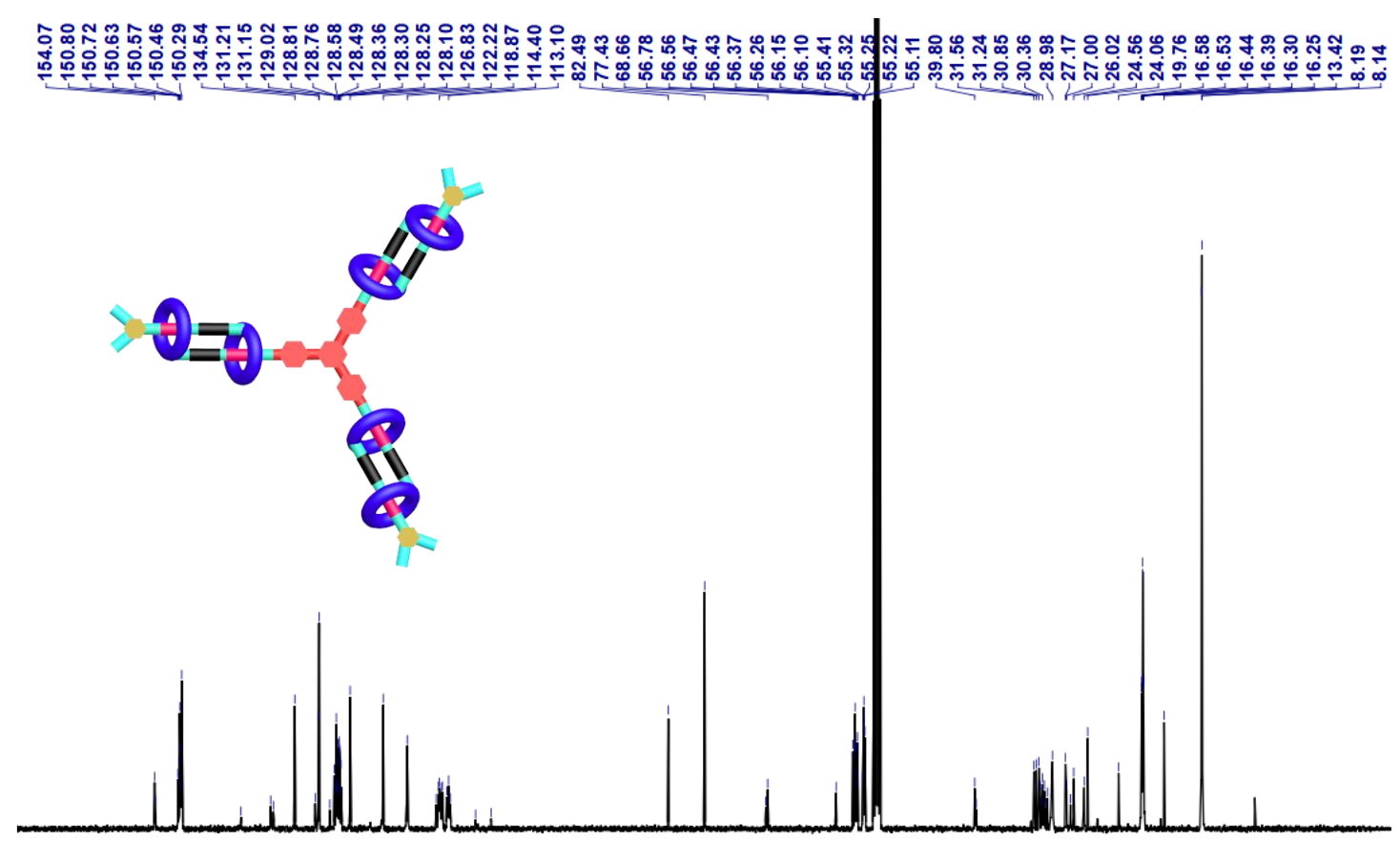

$\begin{array}{lllllllllllllllll}170 & 160 & 150 & 140 & 130 & 120 & 110 & 100 & \begin{array}{c}90 \\ \text { Chemical shift (ppm) }\end{array} & 50 & 40 & 30 & 20 & 10 & 0 & -10\end{array}$

Figure S29: ${ }^{13} \mathrm{C}$ NMR spectrum $\left(\mathrm{CD}_{2} \mathrm{Cl}_{2}, 298 \mathrm{~K}, 126 \mathrm{MHz}\right)$ of the daisy chain dendrimer DC-G1-YNE.

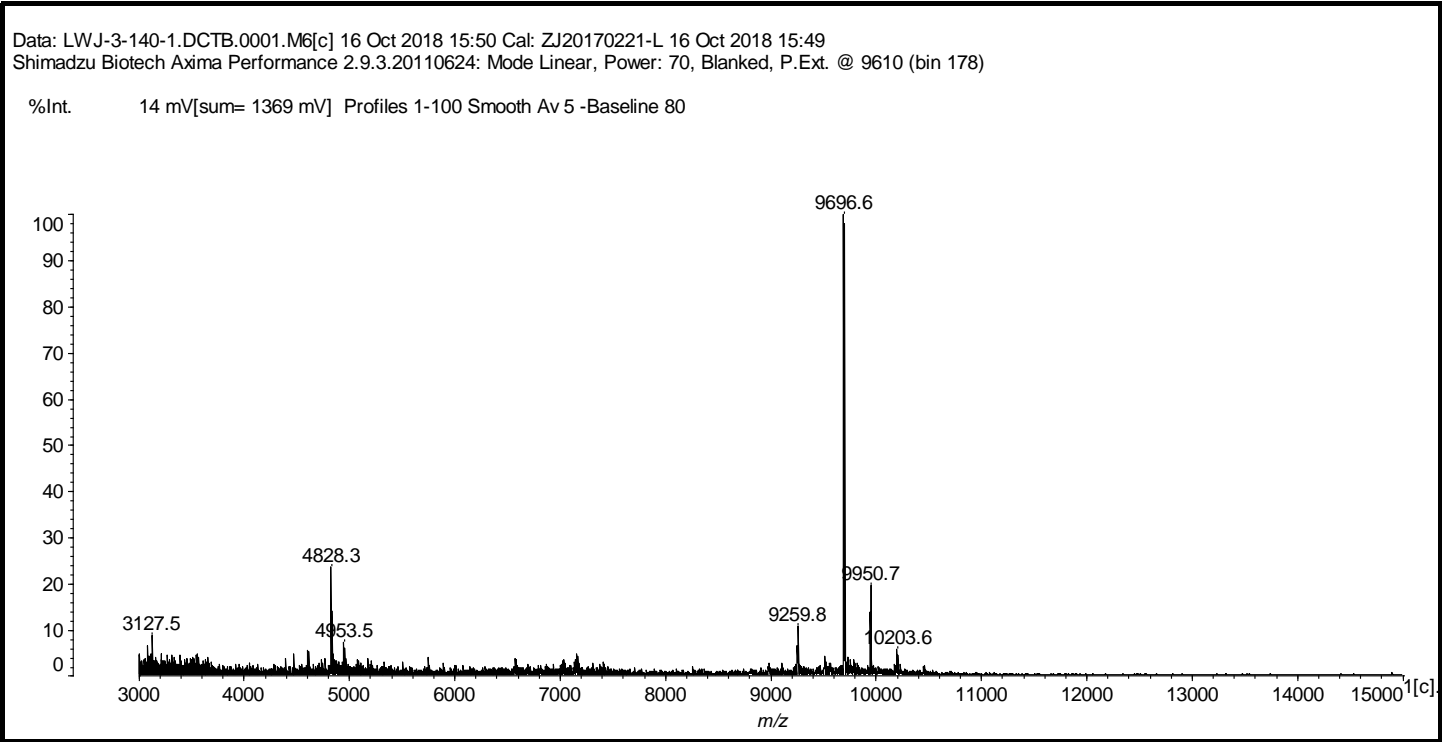

Figure S30: MALDI-TOF-MS spectrum of the daisy chain dendrimer DC-G1-YNE. 


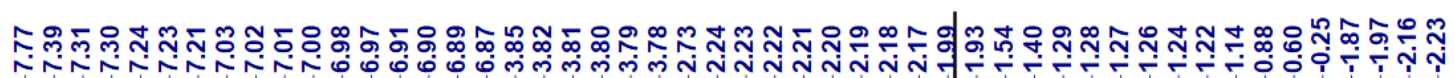
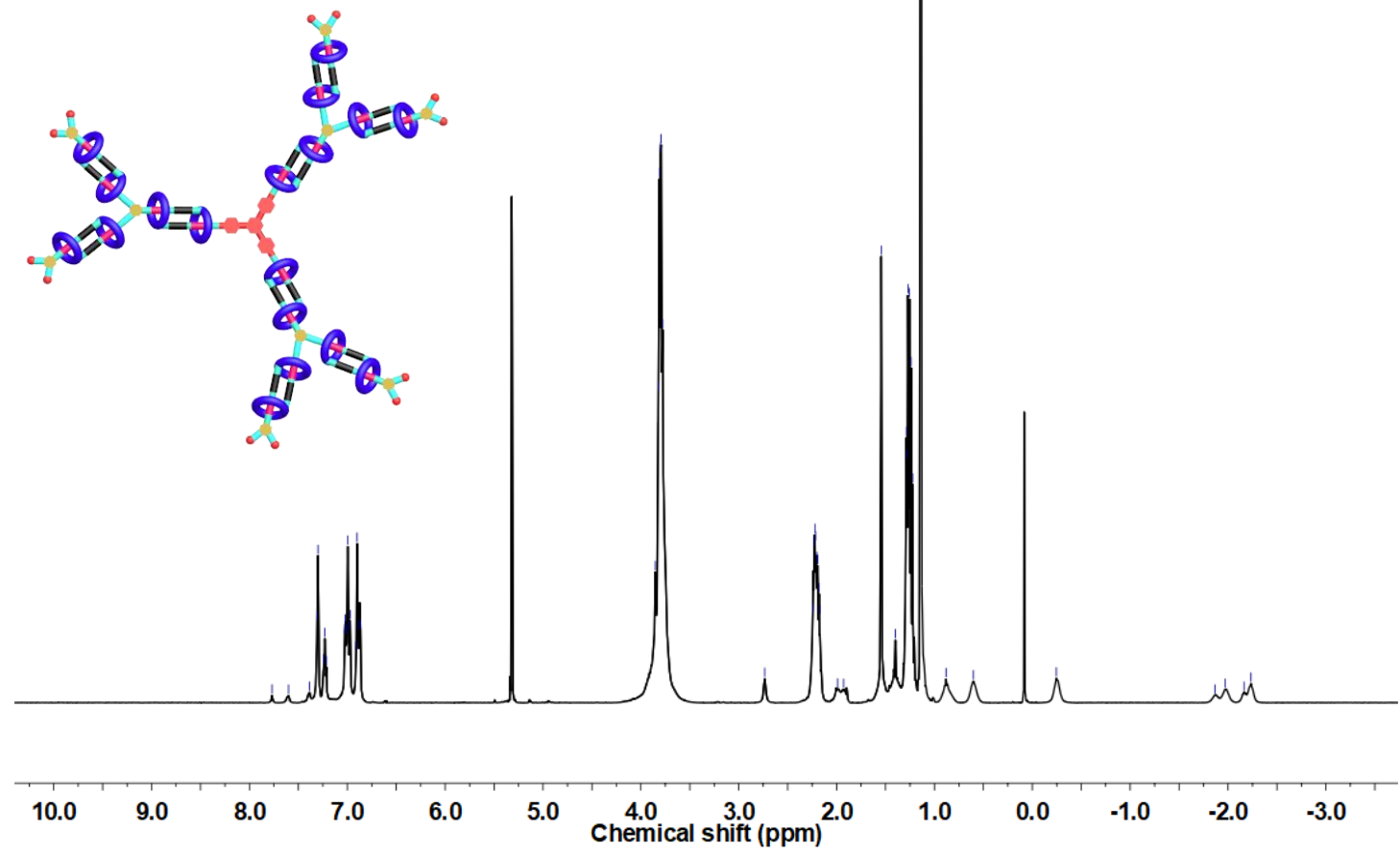

Figure S31: ${ }^{1} \mathrm{H}$ NMR spectrum $\left(\mathrm{CD}_{2} \mathrm{Cl}_{2}, 298 \mathrm{~K}, 500 \mathrm{MHz}\right)$ of the daisy chain dendrimer DC-G2.
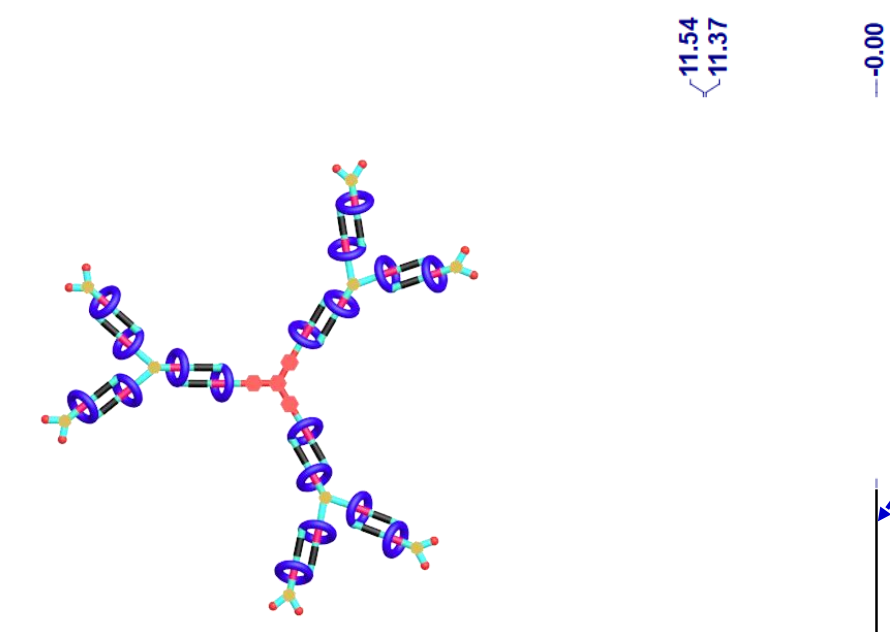

Reference

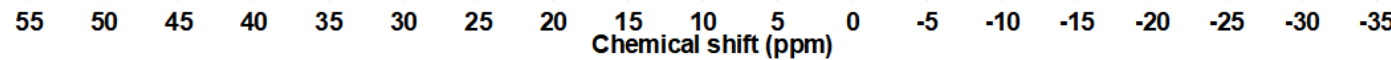

Figure S32: ${ }^{31} \mathrm{P}$ NMR spectrum $\left(\mathrm{CD}_{2} \mathrm{Cl}_{2}, 298 \mathrm{~K}, 202 \mathrm{MHz}\right)$ of the daisy chain dendrimer DC-G2. 


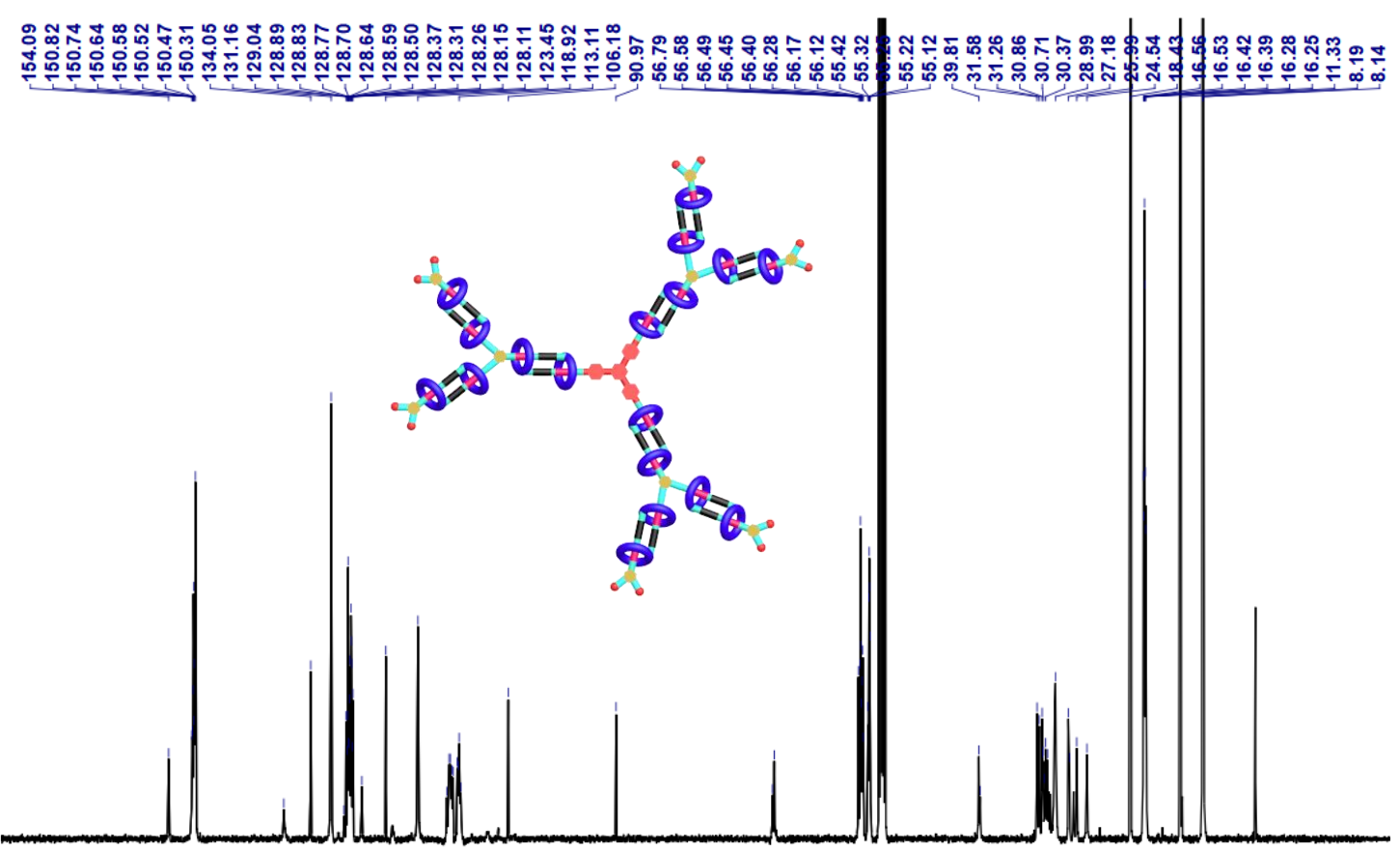

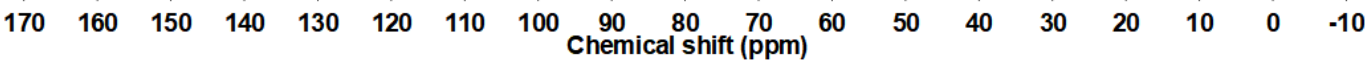

Figure S33: ${ }^{13} \mathrm{C}$ NMR spectrum $\left(\mathrm{CD}_{2} \mathrm{Cl}_{2}, 298 \mathrm{~K}, 126 \mathrm{MHz}\right)$ of the daisy chain dendrimer DC-G2.

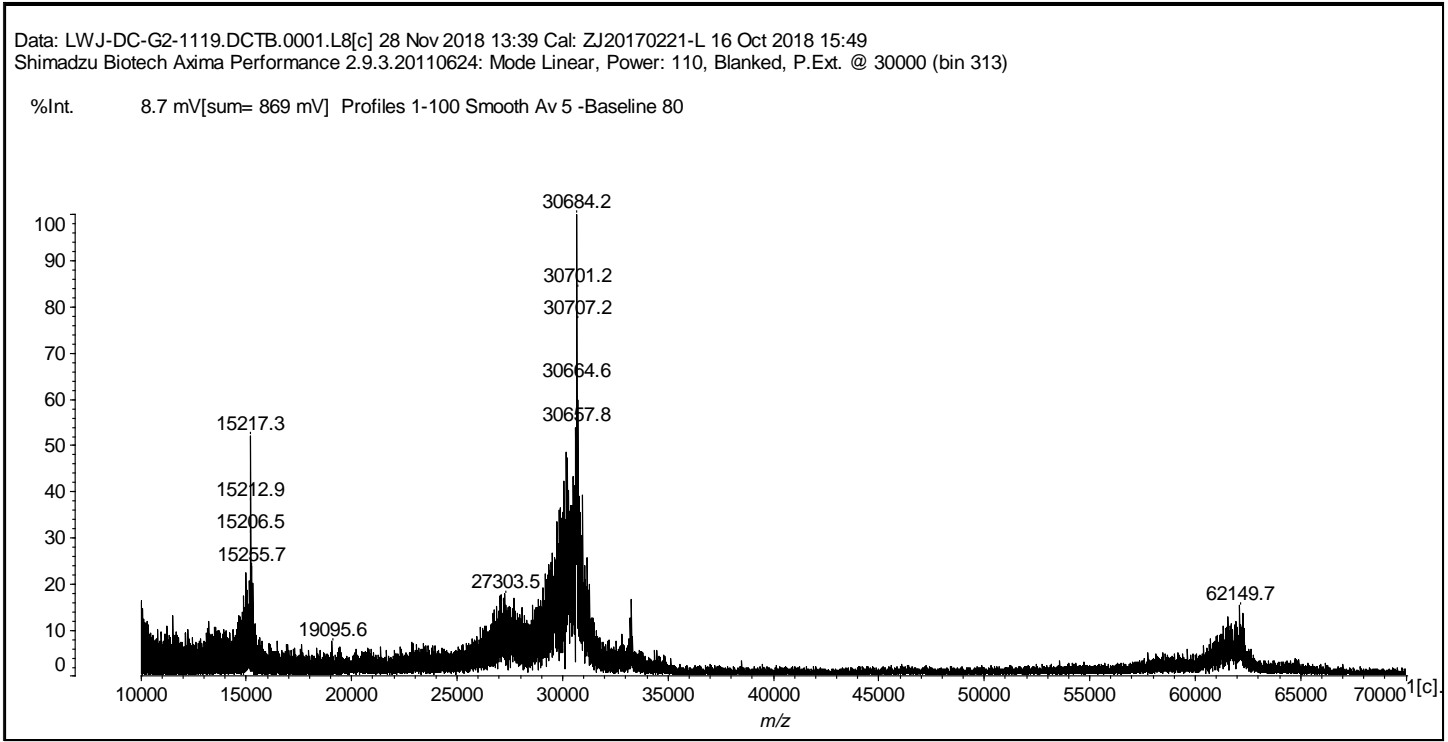

Figure S34: MALDI-TOF-MS spectrum of the daisy chain dendrimer DC-G2. 

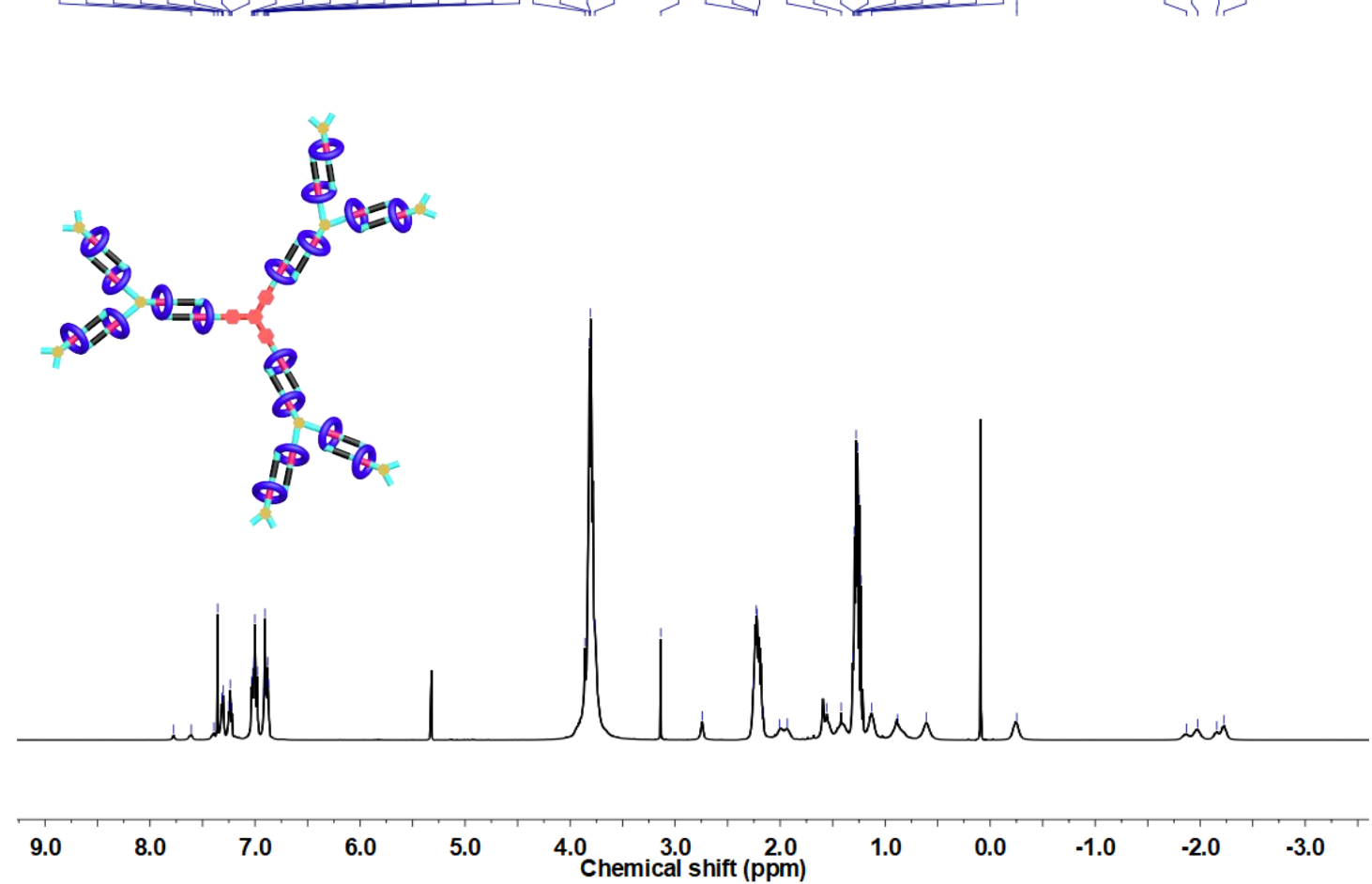

Figure S35: ${ }^{1} \mathrm{H}$ NMR spectrum $\left(\mathrm{CD}_{2} \mathrm{Cl}_{2}, 298 \mathrm{~K}, 500 \mathrm{MHz}\right)$ of the daisy chain dendrimer DC-G2-YNE.
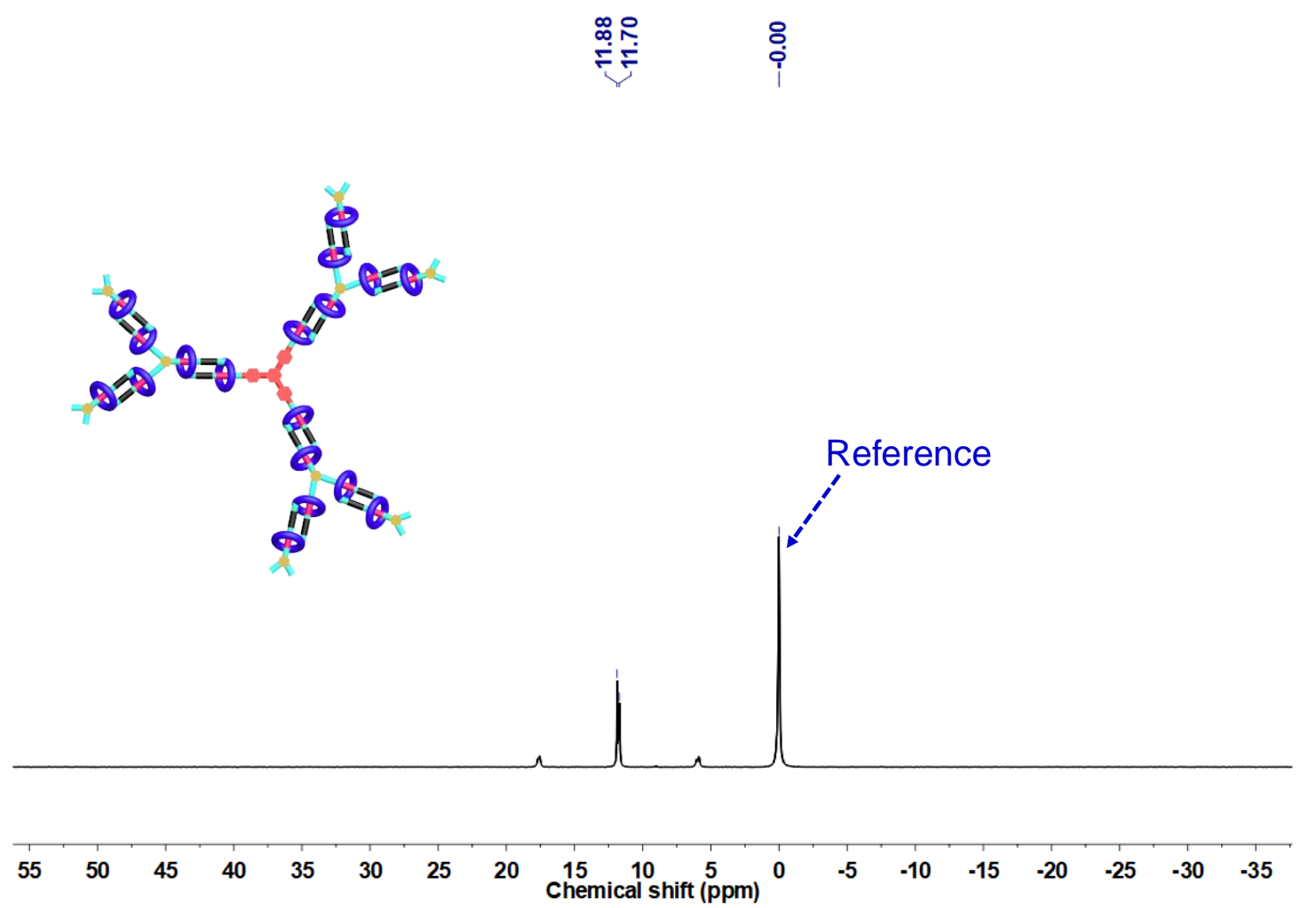

Figure S36: ${ }^{31} \mathrm{P}$ NMR spectrum $\left(\mathrm{CD}_{2} \mathrm{Cl}_{2}, 298 \mathrm{~K}, 202 \mathrm{MHz}\right)$ of the daisy chain dendrimer DC-G2-YNE. 


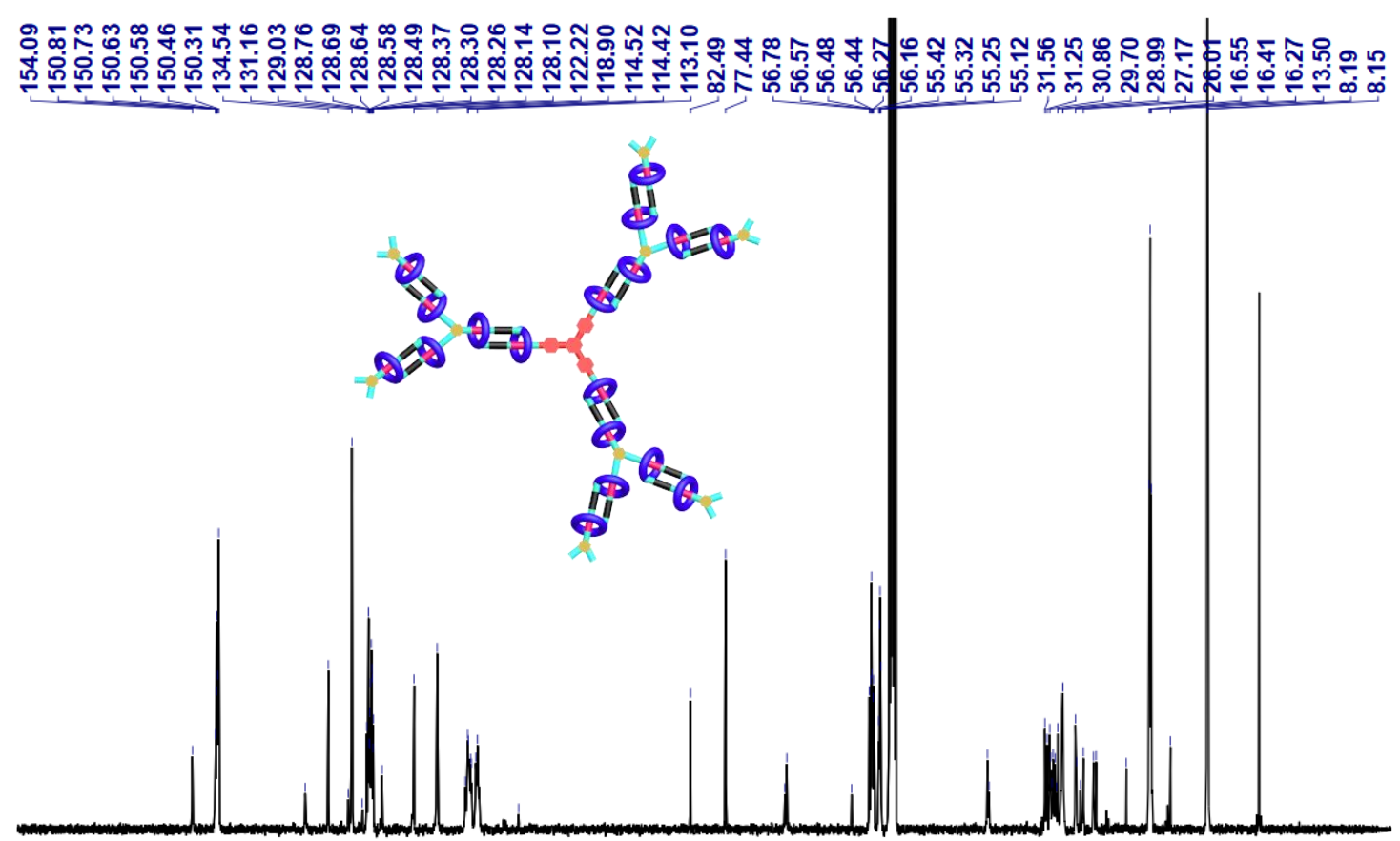

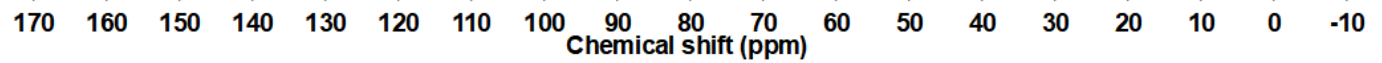

Figure S37: ${ }^{13} \mathrm{C}$ NMR spectrum $\left(\mathrm{CD}_{2} \mathrm{Cl}_{2}, 298 \mathrm{~K}, 126 \mathrm{MHz}\right)$ of the daisy chain dendrimer DC-G2-YNE.

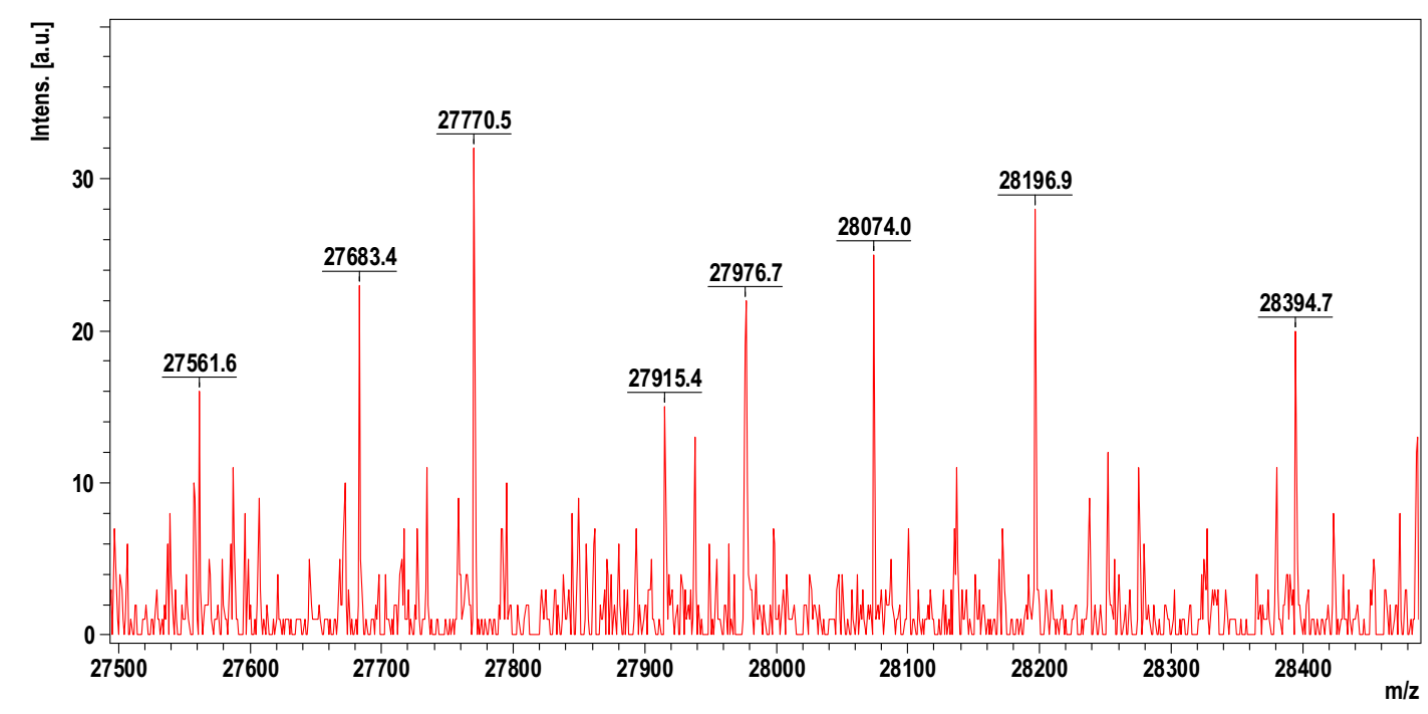

Figure S38: MALDI-TOF-MS spectrum of the daisy chain dendrimer DC-G2-YNE. 


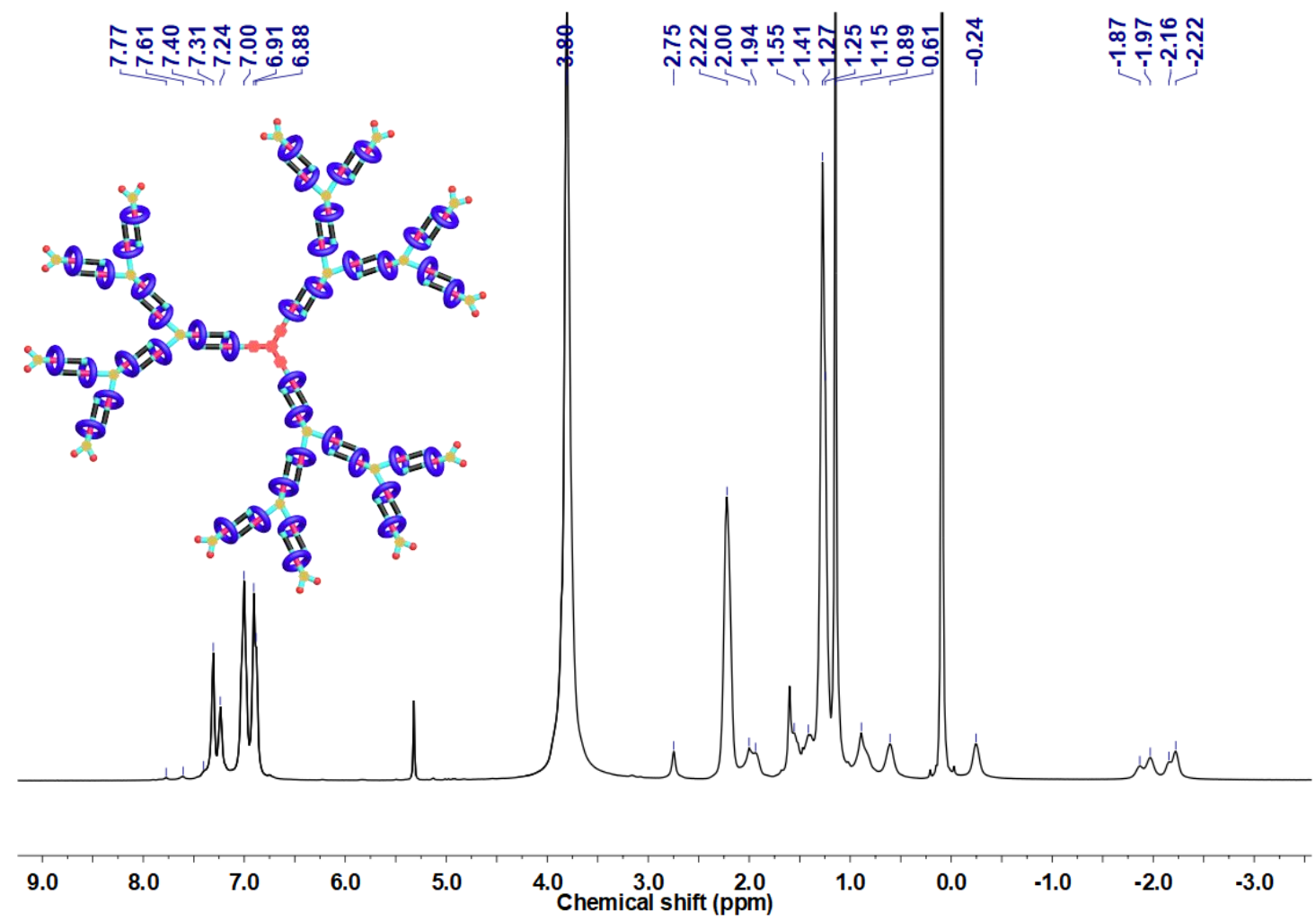

Figure S39: ${ }^{1} \mathrm{H}$ NMR spectrum $\left(\mathrm{CD}_{2} \mathrm{Cl}_{2}, 298 \mathrm{~K}, 500 \mathrm{MHz}\right)$ of the daisy chain dendrimer DC-G3.

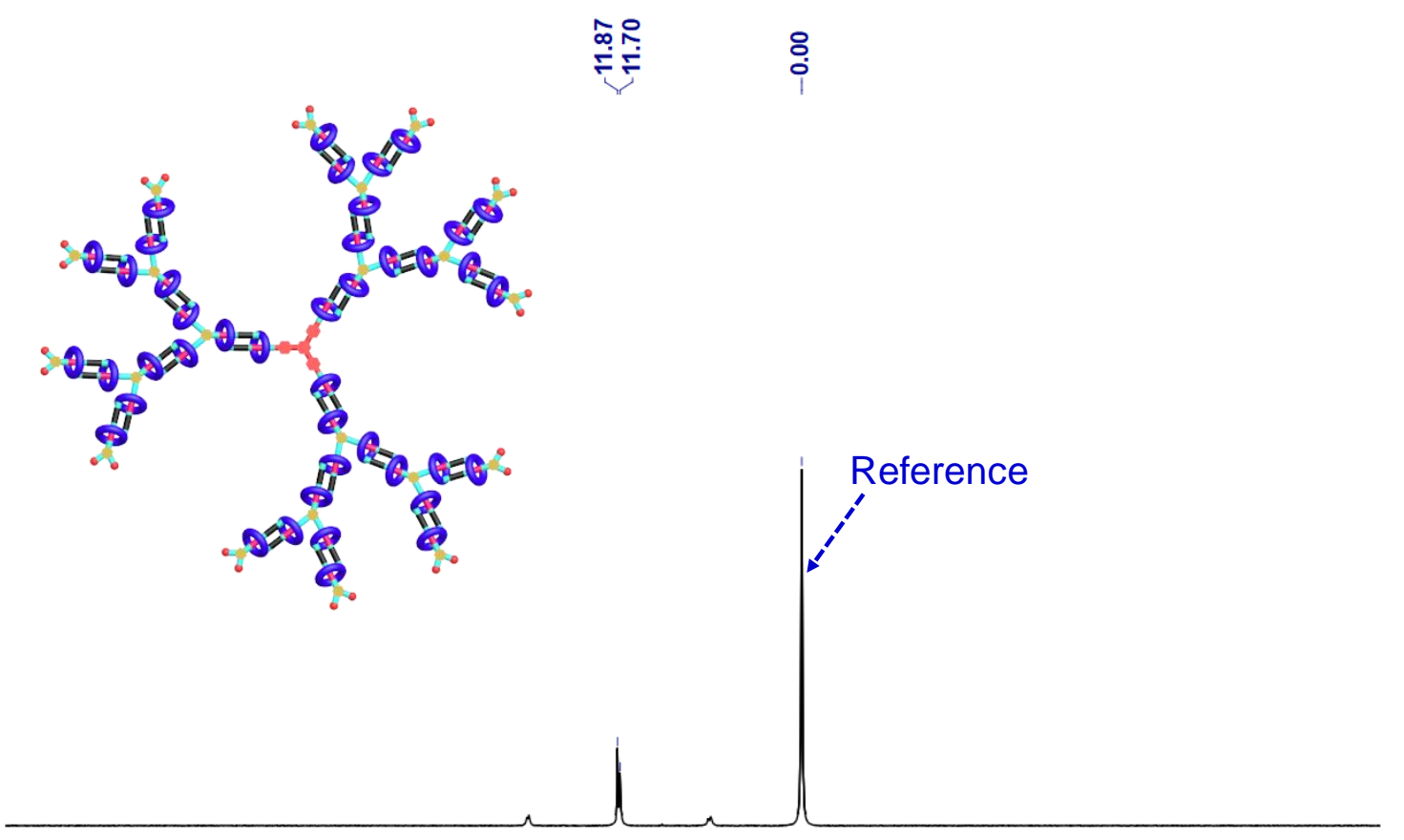

$\begin{array}{lllllllllllllllll}50 & 45 & 40 & 35 & 30 & 25 & 20 & 15 & \begin{array}{c}10 \\ \text { Chemical shift (ppm) }\end{array} & -5 & -10 & -15 & -20 & -25 & -30 & -35\end{array}$

Figure S40: ${ }^{31} \mathrm{P}$ NMR spectrum $\left(\mathrm{CD}_{2} \mathrm{Cl}_{2}, 298 \mathrm{~K}, 202 \mathrm{MHz}\right)$ of the daisy chain dendrimer DC-G3. 


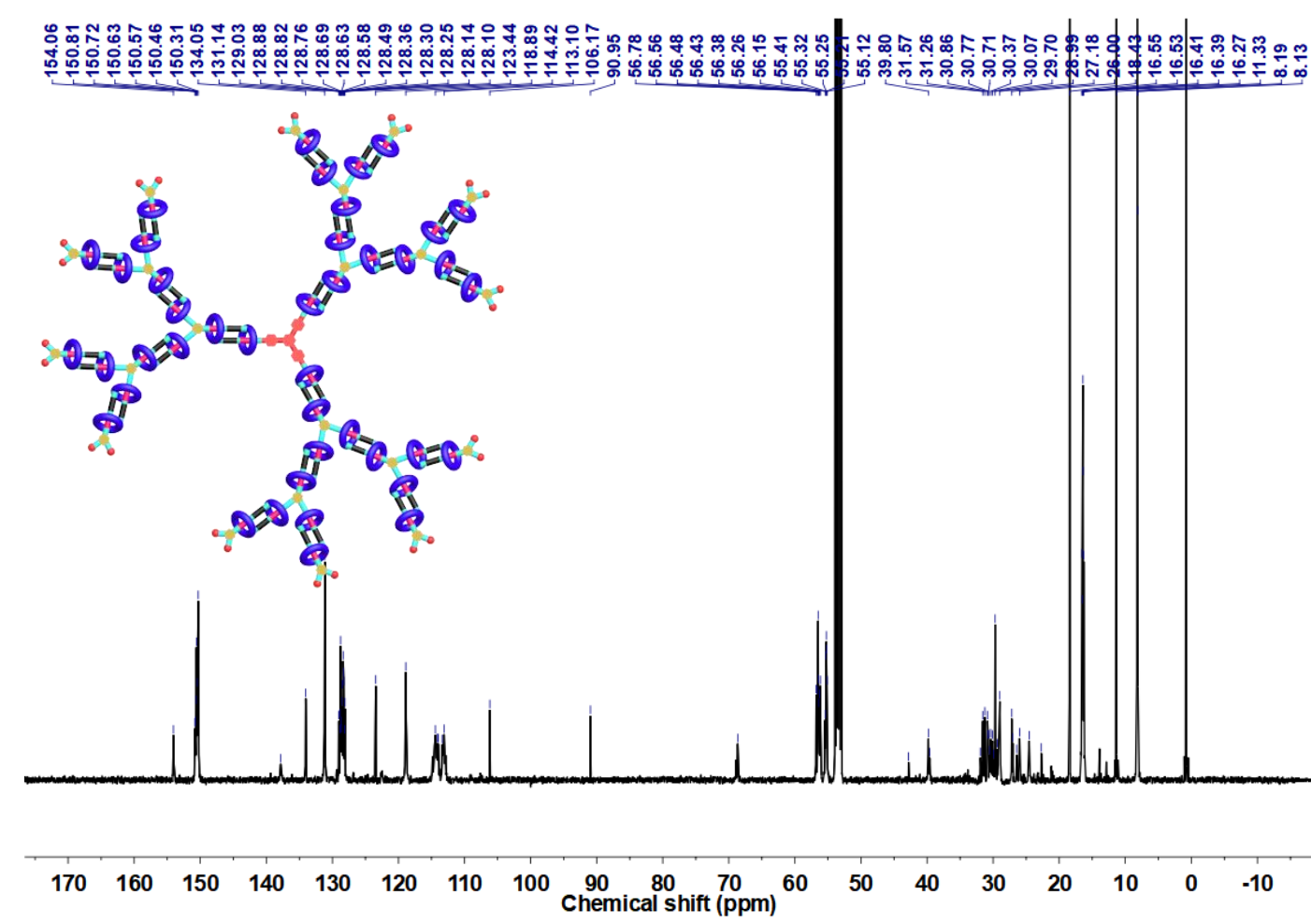

Figure S41: ${ }^{13} \mathrm{C}$ NMR spectrum $\left(\mathrm{CD}_{2} \mathrm{Cl}_{2}, 298 \mathrm{~K}, 126 \mathrm{MHz}\right)$ of the daisy chain dendrimer DC-G3.

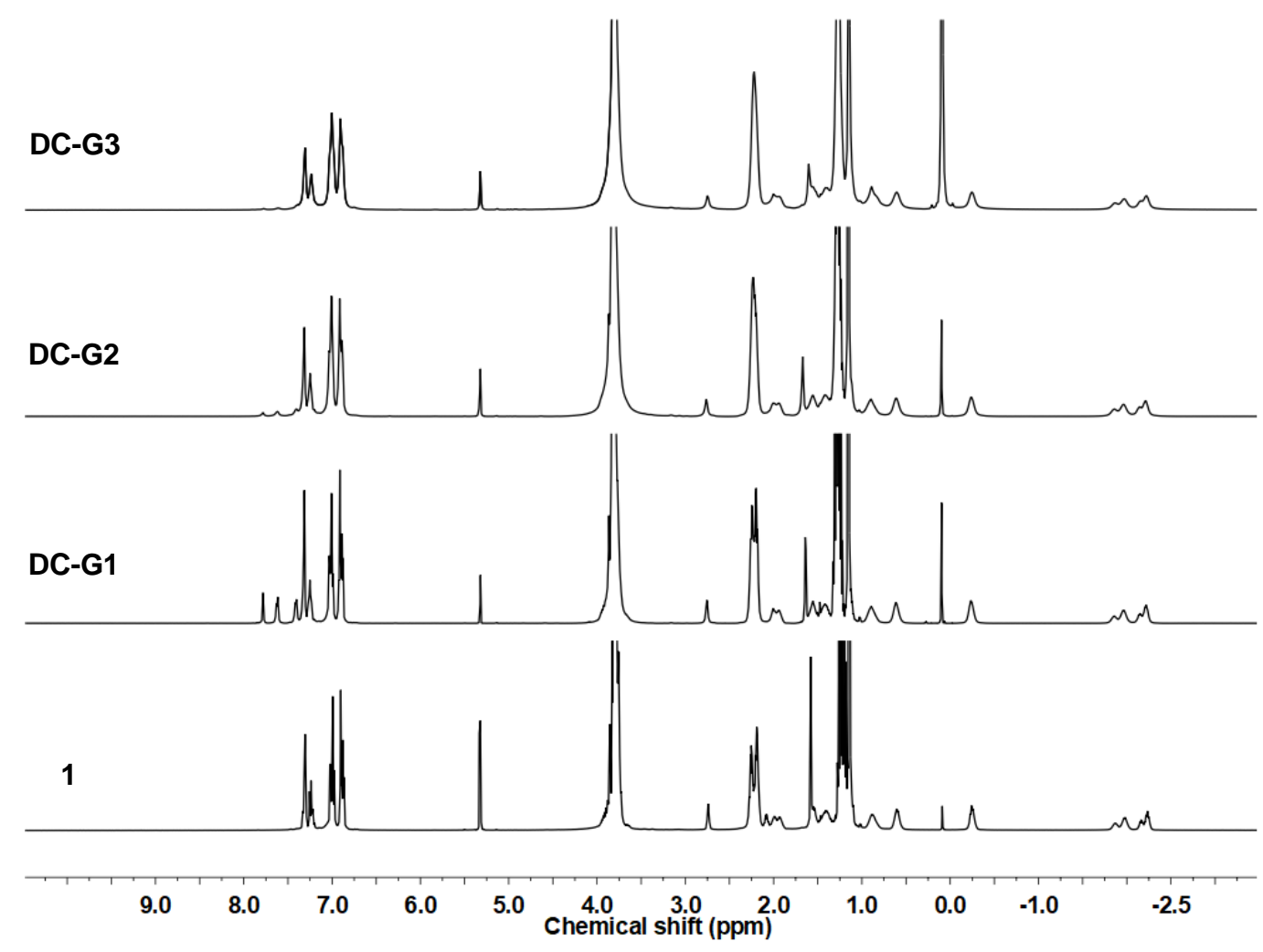

Figure S42: ${ }^{1} \mathrm{H}$ NMR spectra $\left(\mathrm{CD}_{2} \mathrm{Cl}_{2}, 298 \mathrm{~K}, 500 \mathrm{MHz}\right)$ of the daisy chain dendrimers and the [c2]daisy chain rotaxane $\mathbf{1}$. 


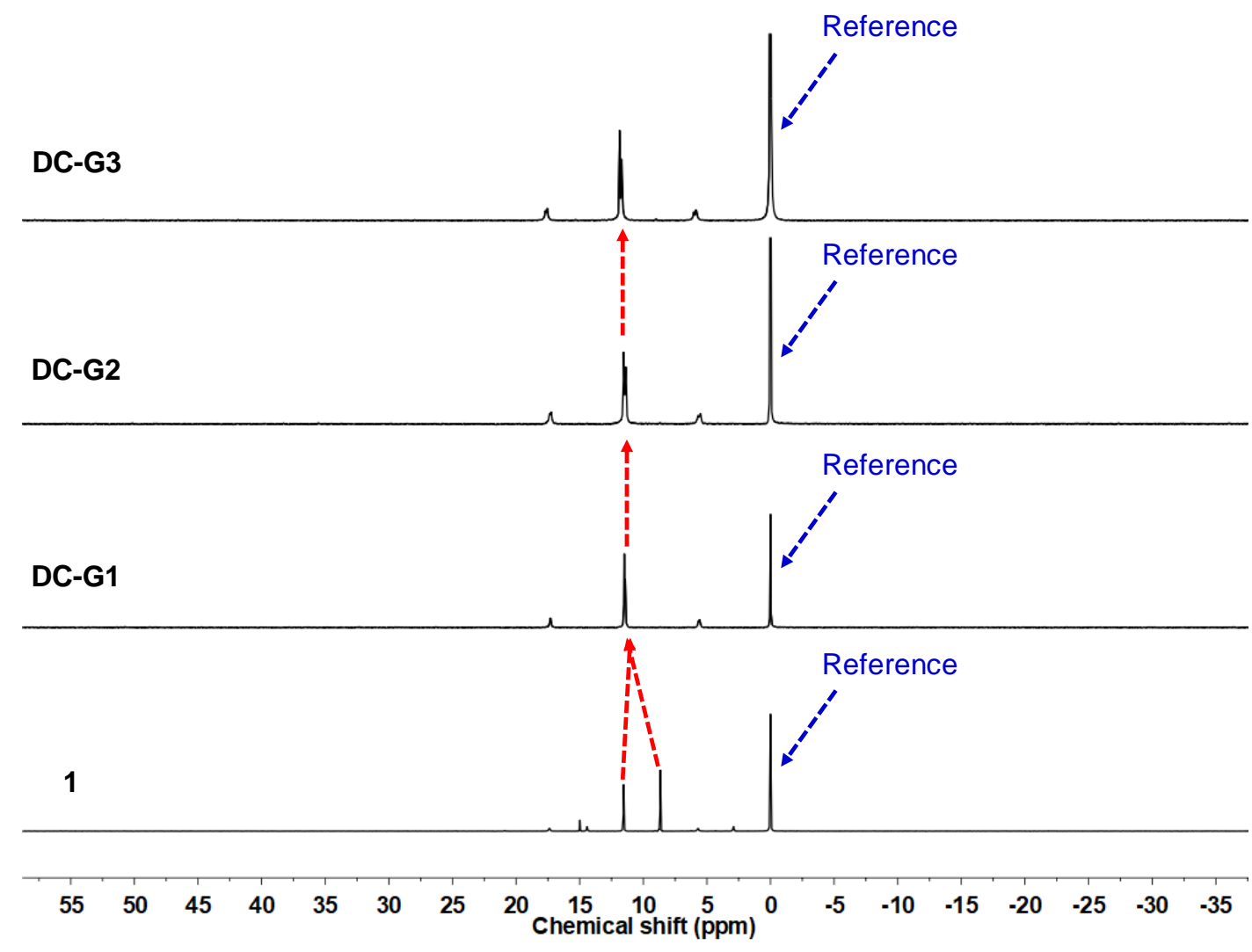

Figure S43: ${ }^{31} \mathrm{P}$ NMR spectra $\left(\mathrm{CD}_{2} \mathrm{Cl}_{2}, 298 \mathrm{~K}, 202 \mathrm{MHz}\right)$ of the daisy chain dendrimers and the [c2]daisy chain rotaxane $\mathbf{1}$.

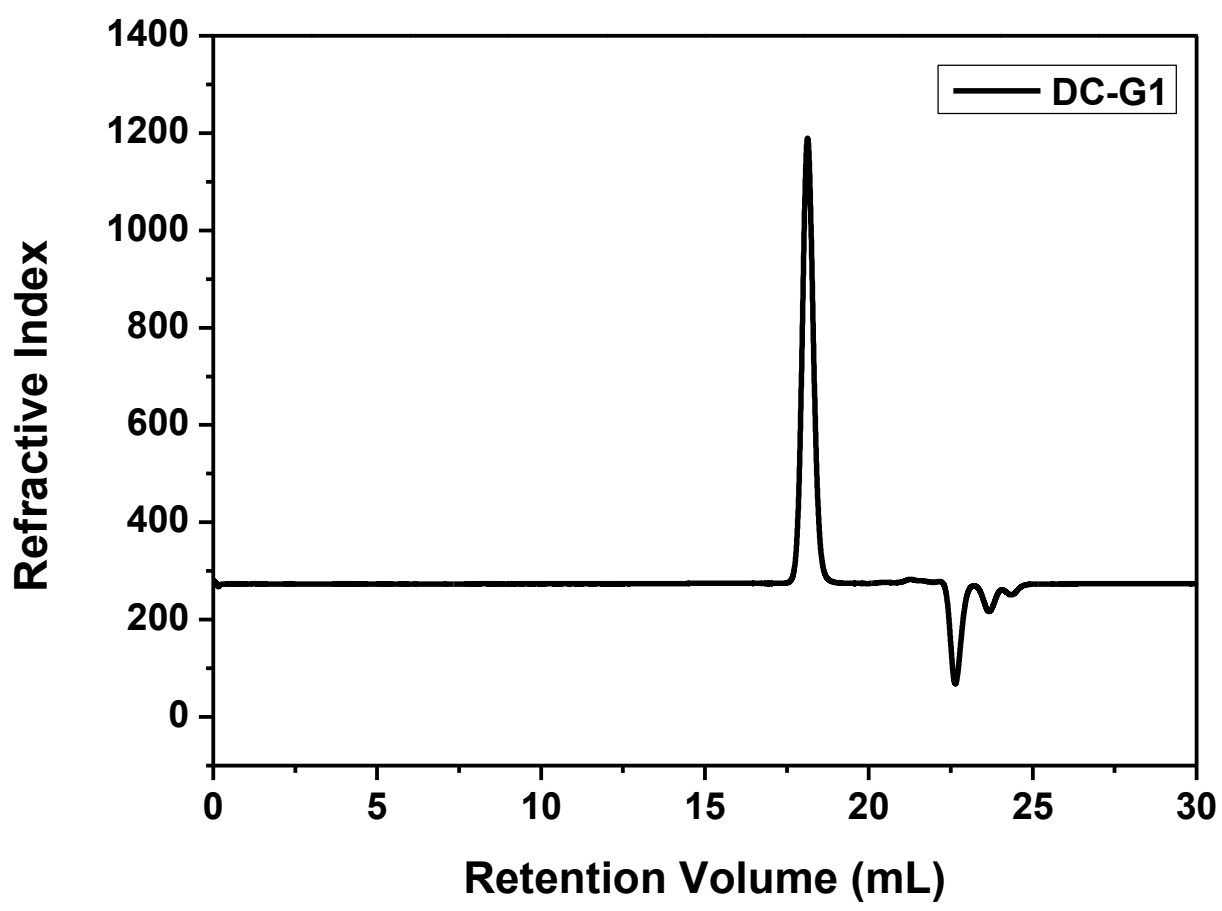

Figure S44: GPC spectrum of the daisy chain dendrimer DC-G1. 


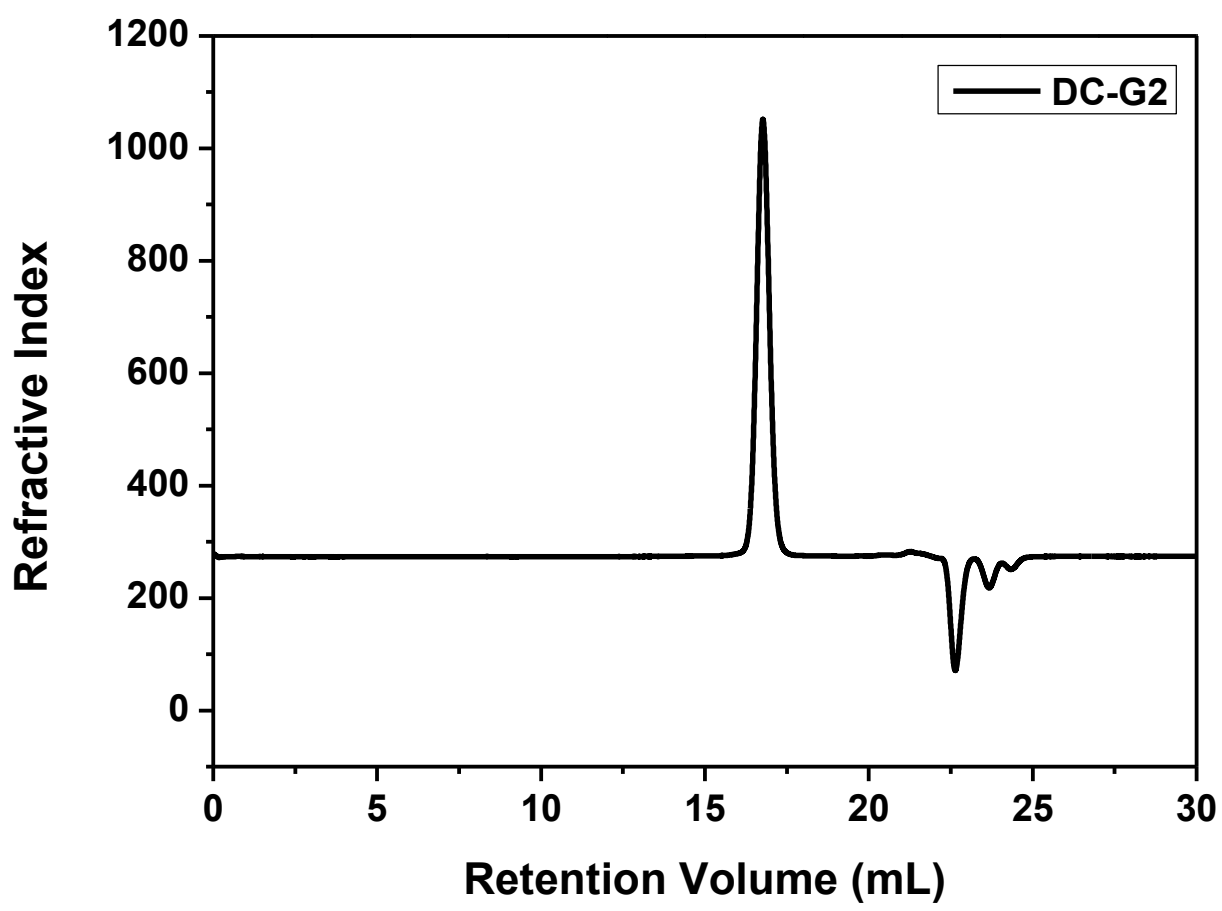

Figure S45: GPC spectrum of the daisy chain dendrimer DC-G2.

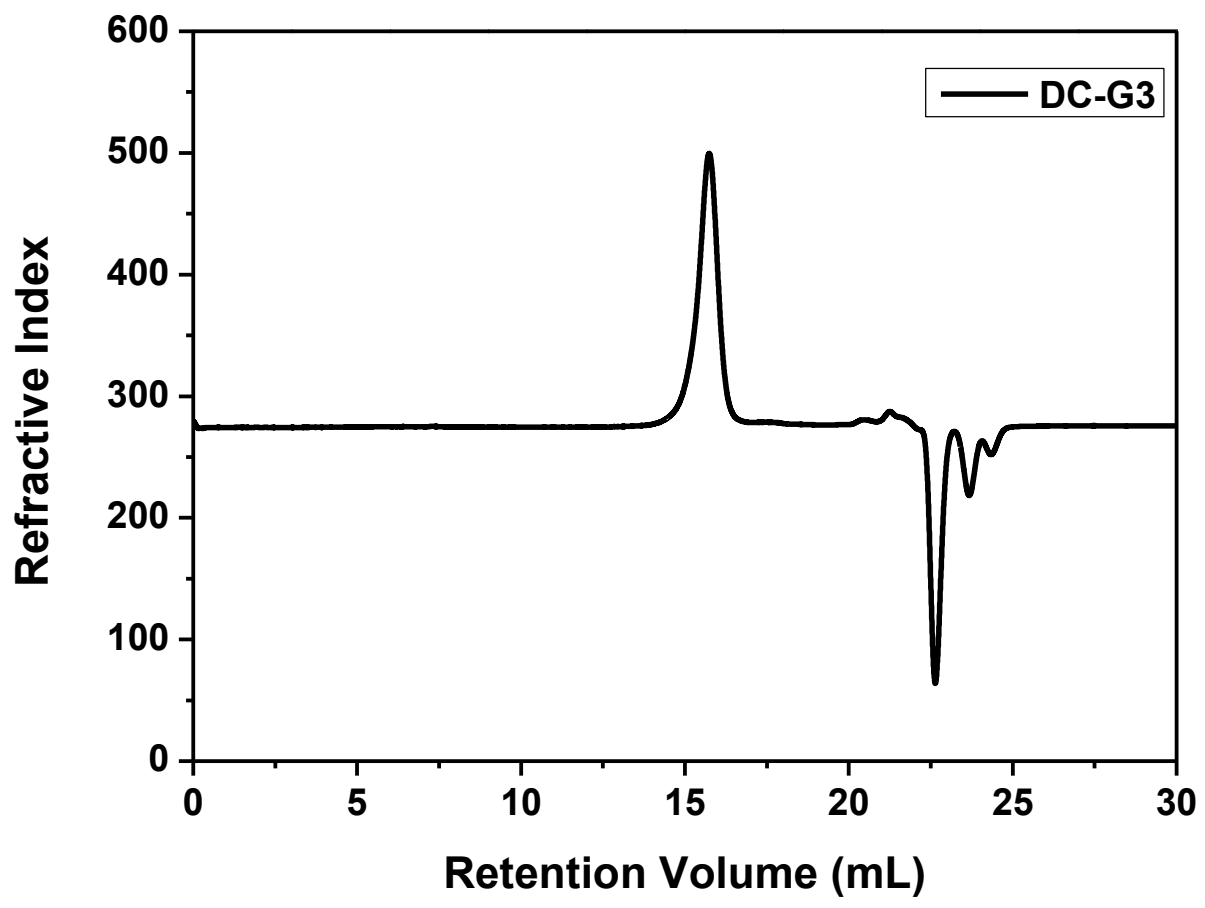

Figure S46: GPC spectrum of the daisy chain dendrimer DC-G3. 


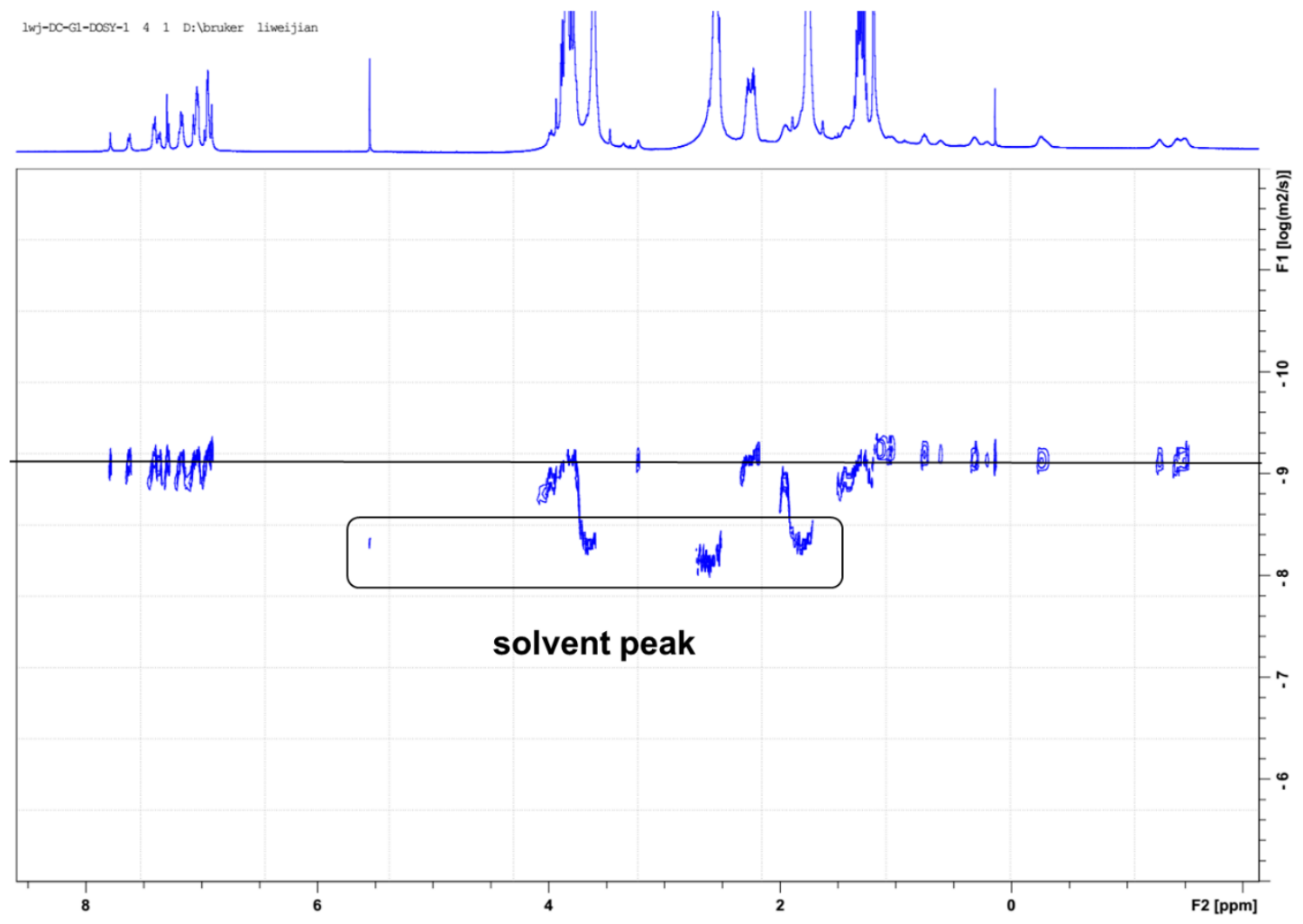

Figure S47: 2-D DOSY spectrum (THF- $d_{8}, 298 \mathrm{~K}, 500 \mathrm{MHz}$ ) of the daisy chain dendrimer DC-G1.

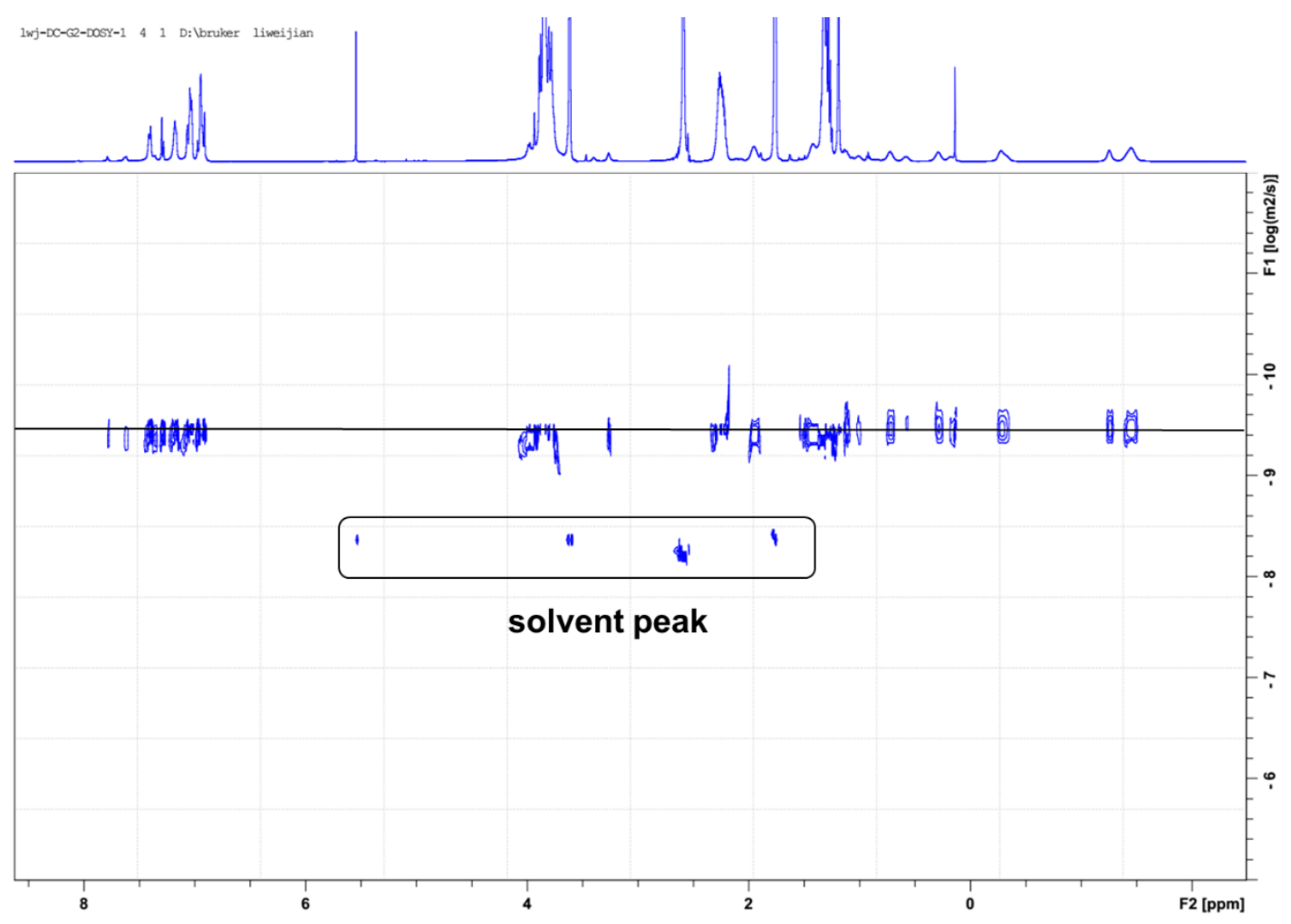

Figure S48: 2-D DOSY spectrum (THF- $d_{8}, 298 \mathrm{~K}, 500 \mathrm{MHz}$ ) of the daisy chain dendrimer DC-G2. 


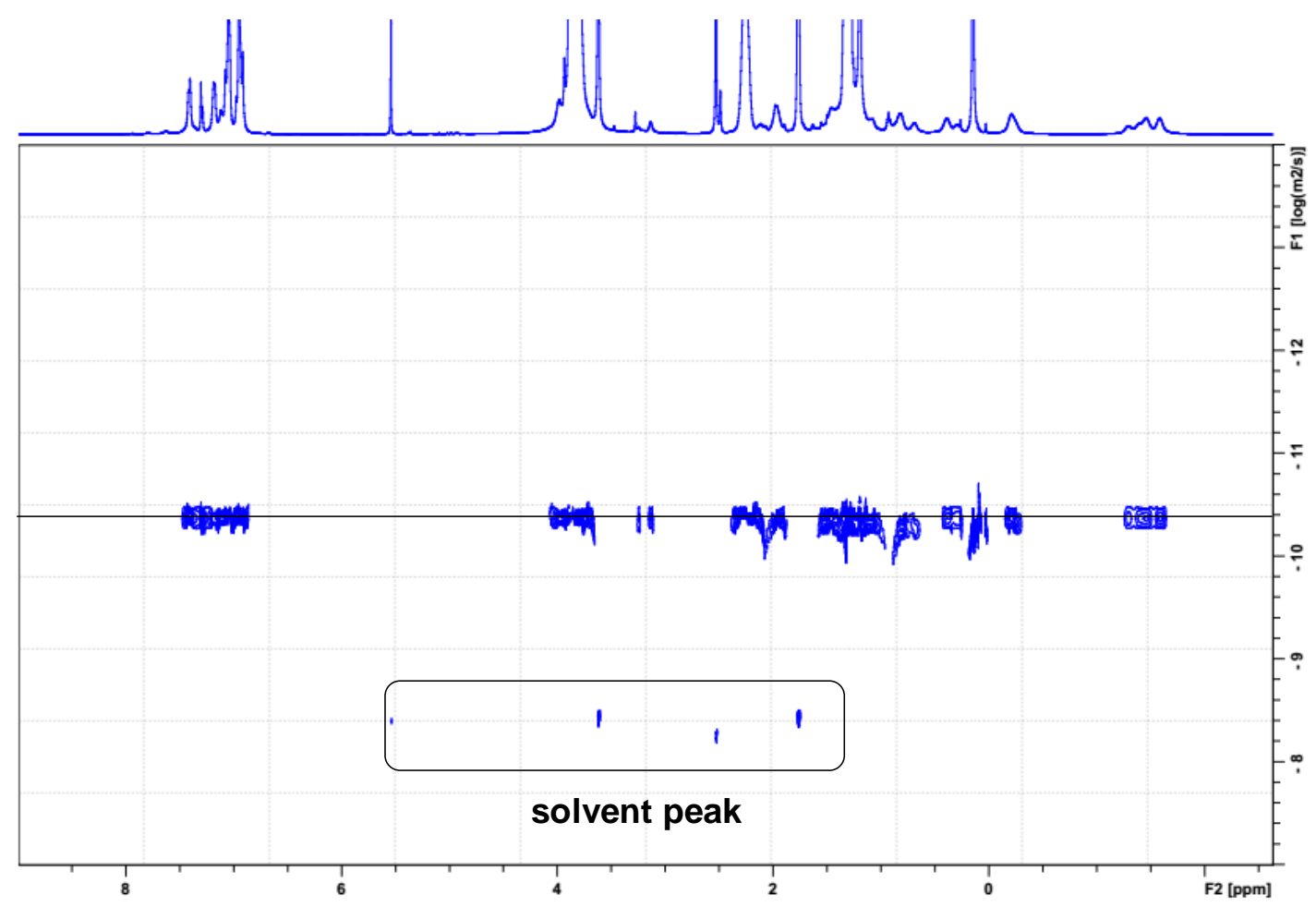

Figure S49: 2-D DOSY spectrum (THF- $d_{8}, 298 \mathrm{~K}, 500 \mathrm{MHz}$ ) of the daisy chain dendrimer DC-G3.

(a)

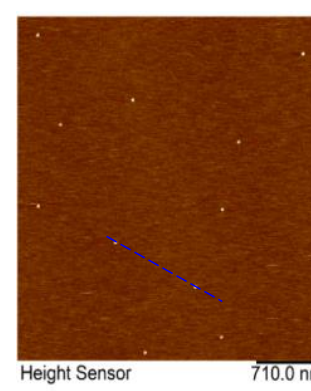

(b)

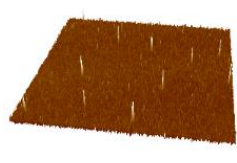

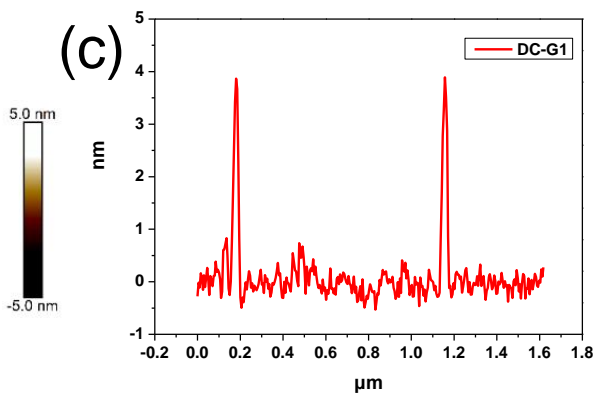

Figure S50: (a-b) AFM image and (c) height distributions of the daisy chain dendrimer DC-G1. The height range is $3.9 \pm 0.1 \mathrm{~nm}$.

(a)

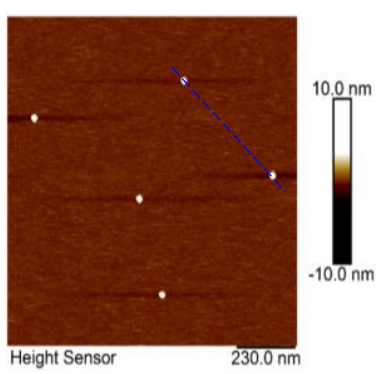

(b)

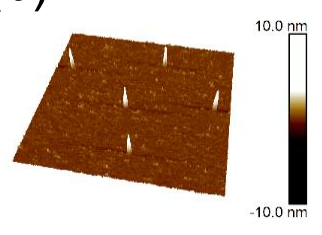

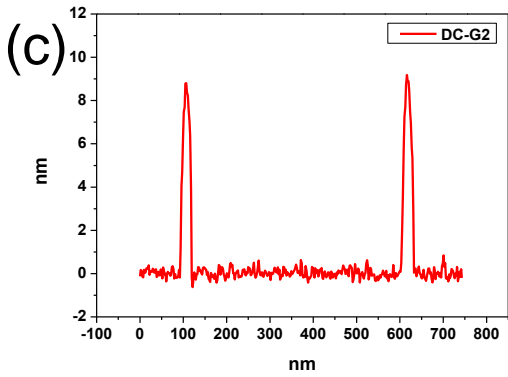

Figure S51: (a-b) AFM image and (c) height distributions of the daisy chain dendrimer DC-G2. The height range is $8.9 \pm 0.2 \mathrm{~nm}$. 
(a)

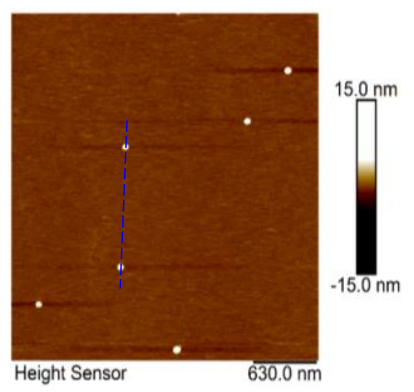

(b)

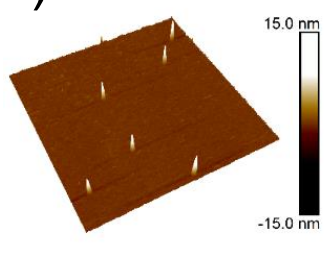

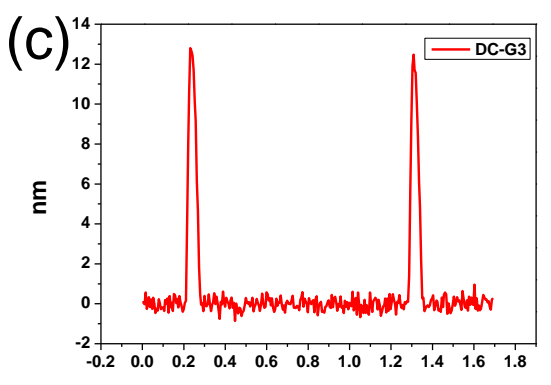

$\mu \mathrm{m}$

Figure S52: (a-b) AFM image and (c) height distributions of the daisy chain dendrimer DC-G3. The height range is $12.8 \pm 0.1 \mathrm{~nm}$.

(a)

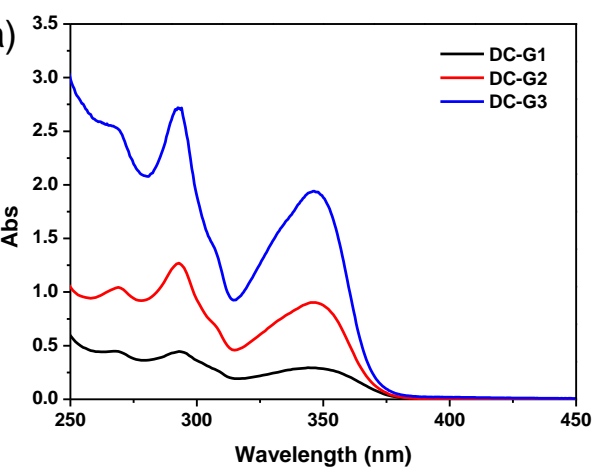

(b)

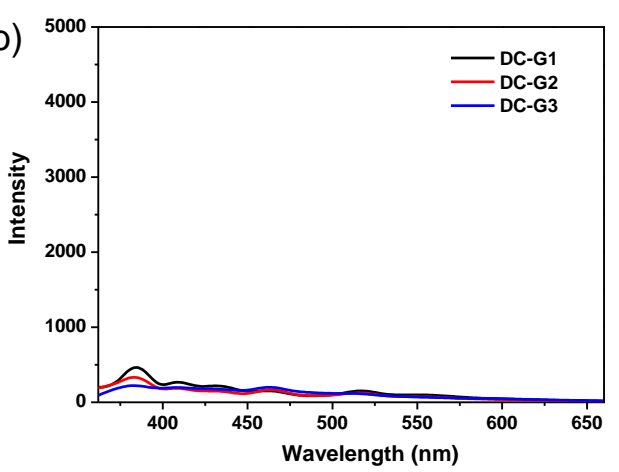

Figure S53: (a) UV-Vis spectra and (b) Emission spectra of the daisy chain dendrimers in THF $\left(10^{-6} \mathrm{M}, \lambda_{\mathrm{ex}}=346 \mathrm{~nm}\right)$. (Since the platinum-acetylide units in the resultant daisy chain dendrimers were well separated, no significant shifts of the $\lambda_{\max }$ values were observed, which indicated that the interaction among the inserted platinum-acetylide moieties was relatively weak. In addition, according to the intensity in the fluorescence spectra, all the daisy chain dendrimers only exhibited very weak luminescence. This finding was not surprising since even the conjugated organometallic dendrimers based on the same linear neutral platinum-acetylide moieties usually displayed weak luminescence.). 
Table S1: Photophysical properties of the daisy chain dendrimers.

\begin{tabular}{|c|c|c|}
\hline Compounds & $\boldsymbol{\lambda}_{\text {abs }}(\mathbf{n m})$ & $\boldsymbol{\varepsilon}\left(\mathbf{M}^{\mathbf{- 1}} \cdot \mathbf{c m}^{-1} \mathbf{)}\right.$ \\
\hline \multirow{2}{*}{ DC-G1 } & 293 & 446000 \\
\cline { 2 - 3 } & 346 & 293000 \\
\hline \multirow{2}{*}{ DC-G2 } & 293 & 1270000 \\
\cline { 2 - 3 } & 346 & 902000 \\
\hline \multirow{2}{*}{ DC-G3 } & 293 & 2716000 \\
\cline { 2 - 3 } & 346 & 1940000 \\
\hline
\end{tabular}

It was found that the growth of the dendrimer generation led to the progression of the molar absorptivity (for $346 \mathrm{~nm}, \varepsilon$ increased from 293000 (DC-G1) to 1940000 (DC-G3); for $293 \mathrm{~nm}, \varepsilon$ increased from 446000 (DC-G1) to 2716000 (DC-G3), respectively) of these absorptions caused by the number increase of platinum-acetylide bonds in the daisy chain dendrimers. 
rotaxane 2

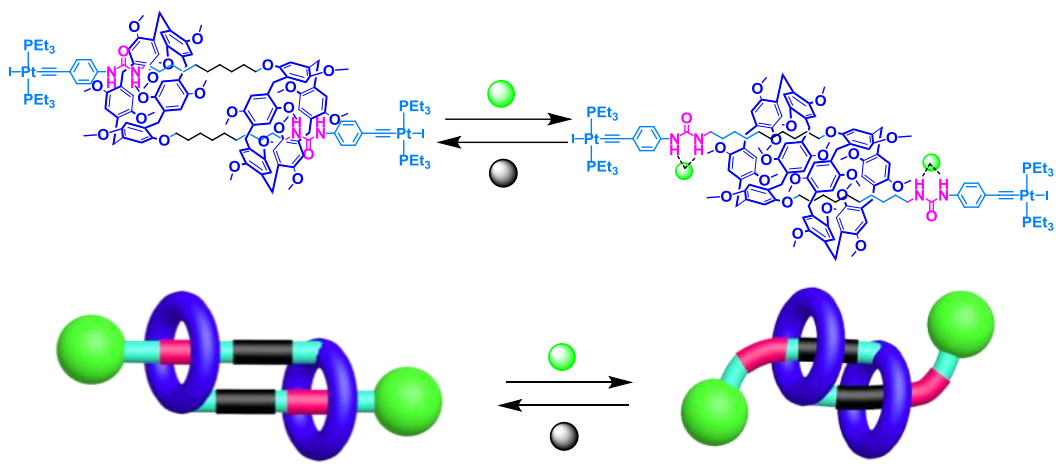

Figure S54: Anion- and solvent-induced switching behavior of the [c2]daisy chain rotaxane 2.

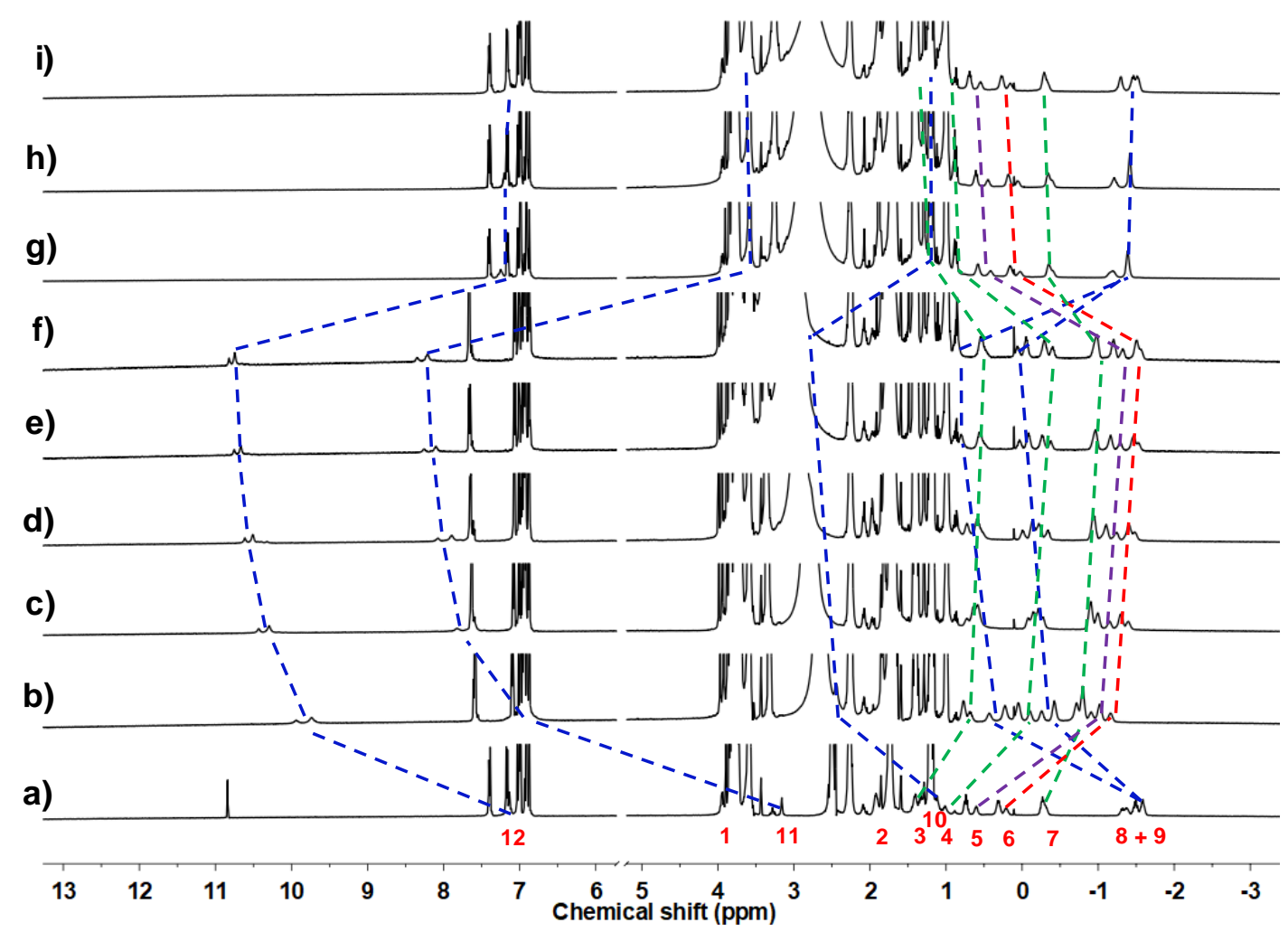

Figure S55: ${ }^{1} \mathrm{H}$ NMR spectra (THF- $d_{8}, 298 \mathrm{~K}, 500 \mathrm{MHz}$ ) of anion-induced switching motion of [c2]daisy chain building block 2. a) 2; the mixture of 2 and TBAA: b) TBAA ( 2 equiv); (c) TBAA (4 equiv); d) TBAA (6 equiv); e) TBAA ( 8 equiv); f) TBAA (10 equiv); and the mixture obtained after adding $\mathrm{NaPF}_{6}$ to the solution in $\mathrm{f}$ ): $\mathrm{g}$ ) $\mathrm{NaPF}_{6}$ (10 equiv); h) $\mathrm{NaPF}_{6}$ (14 equiv); i) $\mathrm{NaPF}_{6}$ (16 equiv). 


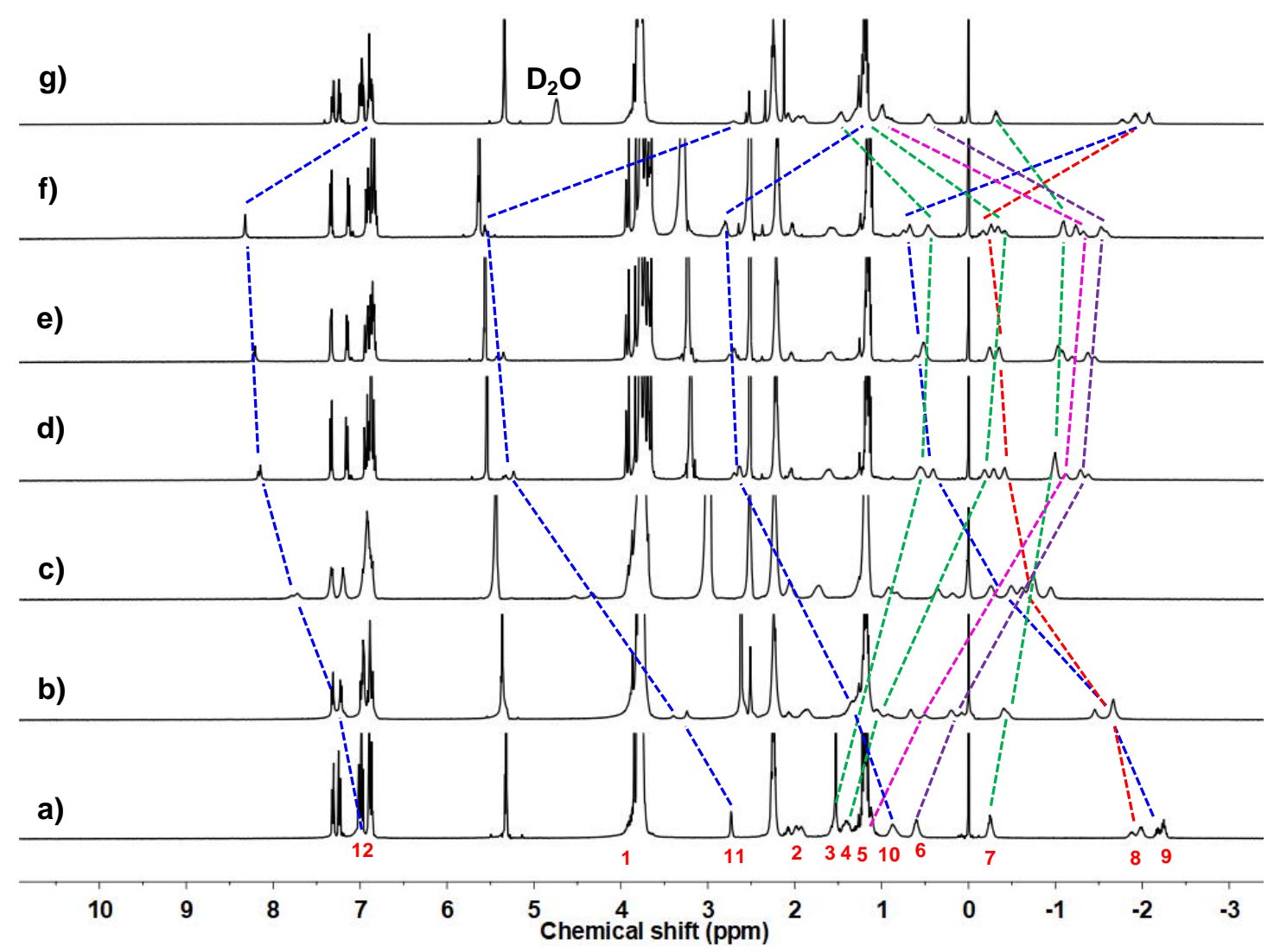

Figure S56: ${ }^{1} \mathrm{H}$ NMR spectra $\left(\mathrm{CD}_{2} \mathrm{Cl}_{2}, 298 \mathrm{~K}, 500 \mathrm{MHz}\right)$ of solvent-induced switching motion of [c2] daisy chain rotaxane 2. a) 2 in $\mathrm{CD}_{2} \mathrm{Cl}_{2}(400 \mu \mathrm{L})$; the addition of DMSO- $d_{6}$ in a): b) $40 \mu \mathrm{L}$; c) $120 \mu \mathrm{L}$; d) $320 \mu \mathrm{L}$; e) $400 \mu \mathrm{L}$; f) $800 \mu \mathrm{L}$; g) the addition of $\mathrm{D}_{2} \mathrm{O}(800 \mu \mathrm{L})$ in $\left.\mathrm{f}\right)$. 


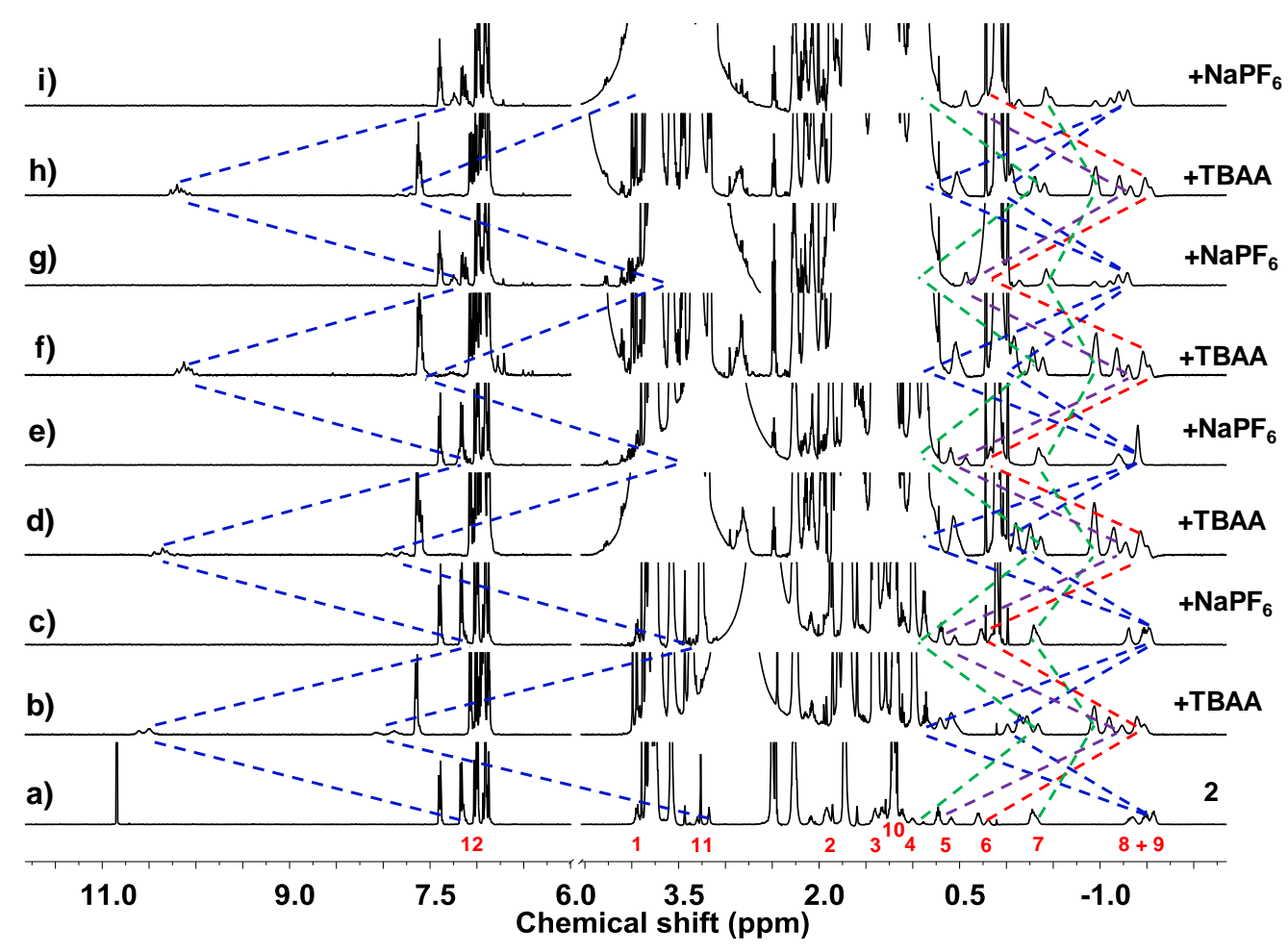

Figure S57: ${ }^{1} \mathrm{H}$ NMR spectra (THF- $d_{8}, 298 \mathrm{~K}, 500 \mathrm{MHz}$ ) of anion-induced switching motion of [c2]daisy chain building block 2 for 4 cycles.

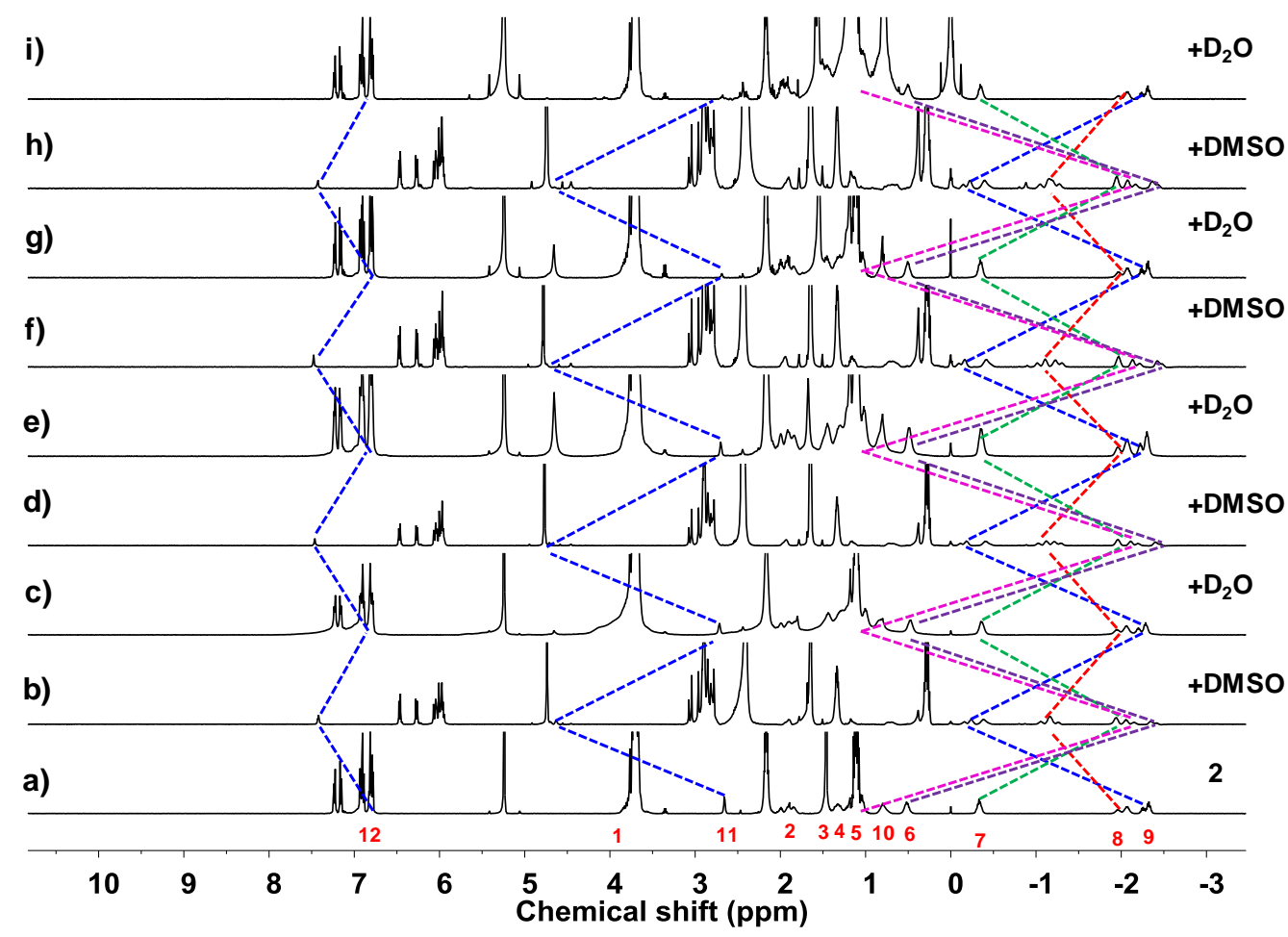

Figure S58: ${ }^{1} \mathrm{H}$ NMR spectra $\left(\mathrm{CD}_{2} \mathrm{Cl}_{2}, 298 \mathrm{~K}, 500 \mathrm{MHz}\right)$ of solvent-induced switching motion of [c2]daisy chain building block 2 for 4 cycles. 


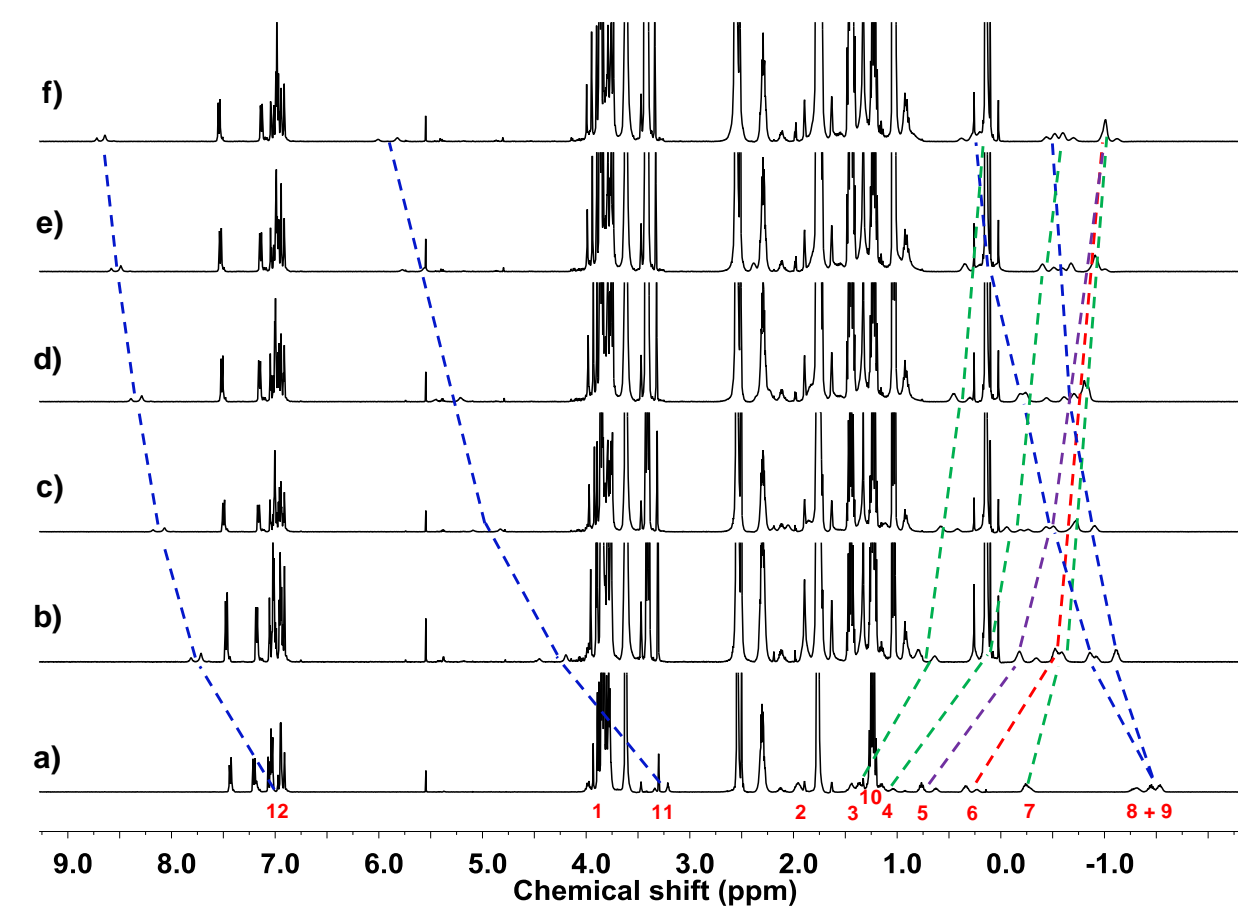

Figure S59: ${ }^{1} \mathrm{H}$ NMR spectra (THF- $d_{8}, 298 \mathrm{~K}, 500 \mathrm{MHz}$ ) of anion-induced switching motion of [c2]daisy chain building block 2 . a) $\mathbf{2}$; the mixture of $\mathbf{2}$ and $\mathrm{TBANO}_{3}$ : b) $\mathrm{TBANO}_{3}$ (2 equiv.); (c) $\mathrm{TBANO}_{3}$ (4 equiv.); d) $\mathrm{TBANO}_{3}$ (6 equiv.); e) $\mathrm{TBANO}_{3}$ (8 equiv.); f) $\mathrm{TBANO}_{3}$ (10 equiv.).

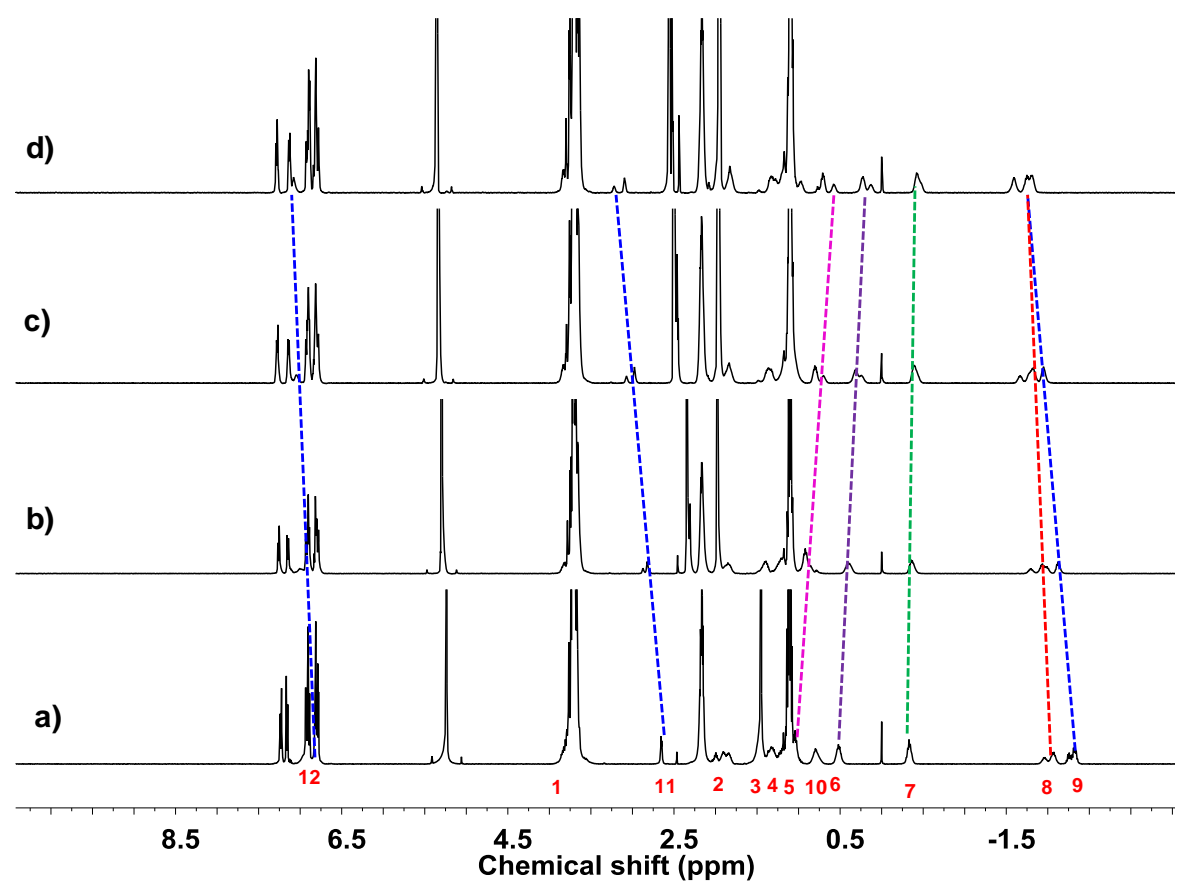

Figure S60: ${ }^{1} \mathrm{H}$ NMR spectra $\left(\mathrm{CD}_{2} \mathrm{Cl}_{2}, 298 \mathrm{~K}, 500 \mathrm{MHz}\right)$ of solvent-induced switching motion of [c2] daisy chain rotaxane 2. a) 2 in $\mathrm{CD}_{2} \mathrm{Cl}_{2}(500 \mu \mathrm{L})$; the addition of acetone- $d_{6}$ in a): b) $200 \mu \mathrm{L}$; c) $400 \mu \mathrm{L}$; d) $600 \mu \mathrm{L}$. 


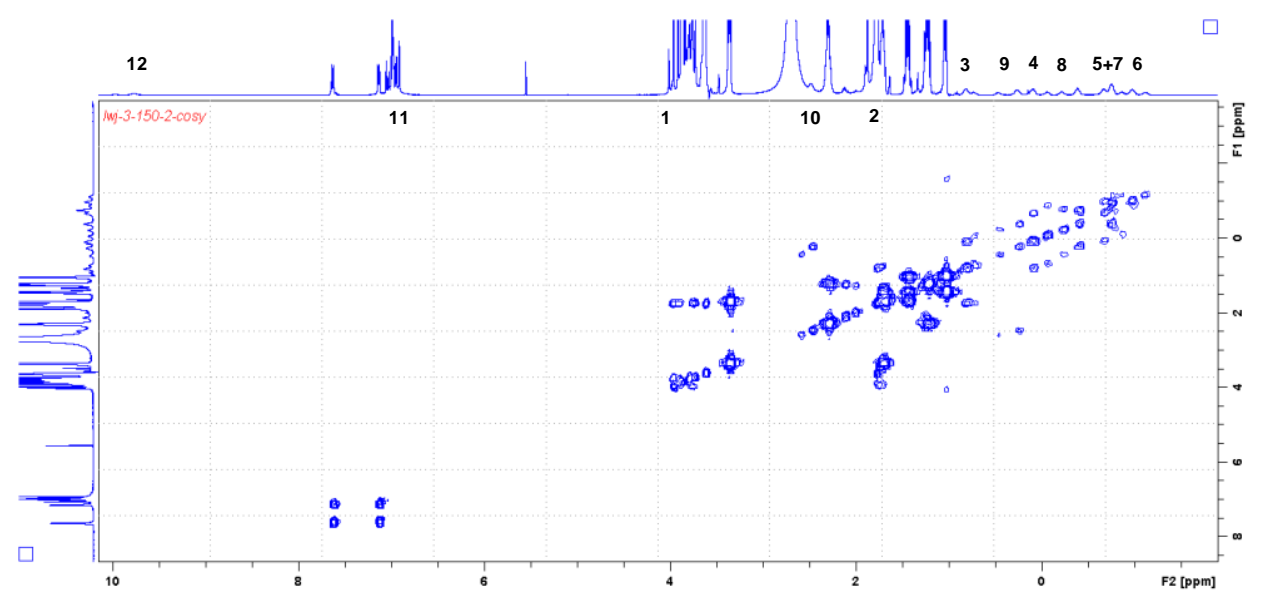

Figure S61: 2D ${ }^{1} \mathrm{H}-{ }^{1} \mathrm{H}$ COSY spectrum (THF- $d_{8}, 298 \mathrm{~K}, 500 \mathrm{MHz}$ ) of [c2] daisy chain rotaxane 2 with the addition of 2 eq. of TBAA.

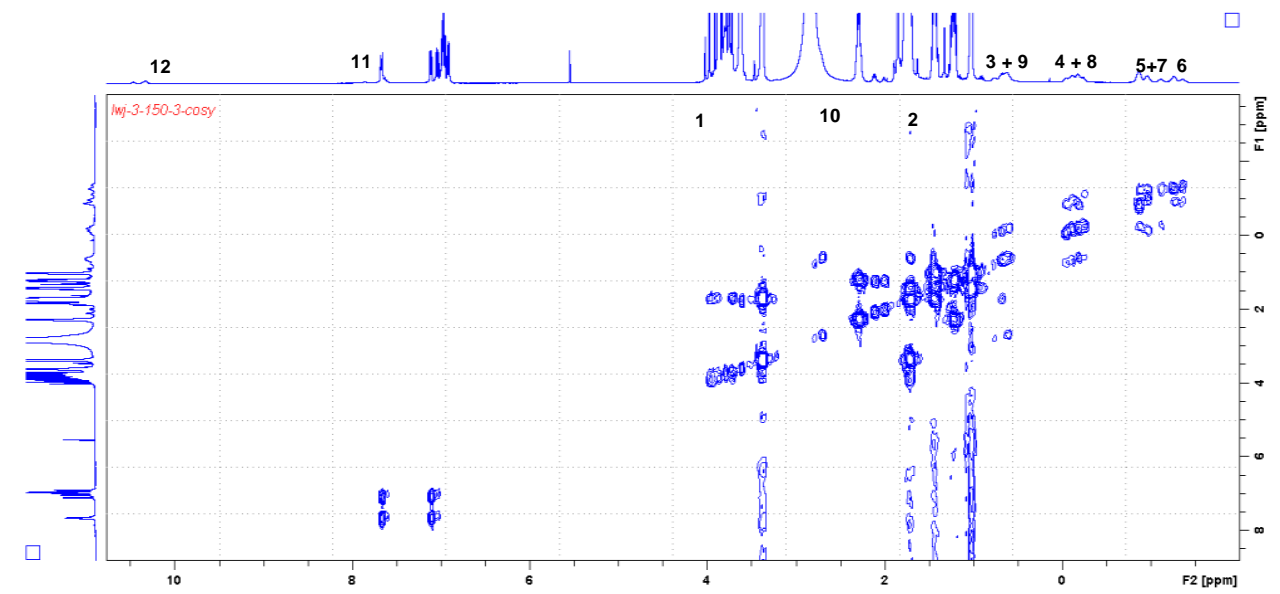

Figure S62: 2D ${ }^{1} \mathrm{H}-{ }^{1} \mathrm{H}$ COSY spectrum (THF- $d_{8}, 298 \mathrm{~K}, 500 \mathrm{MHz}$ ) of [c2]daisy chain rotaxane 2 with the addition of 4 eq. of TBAA.

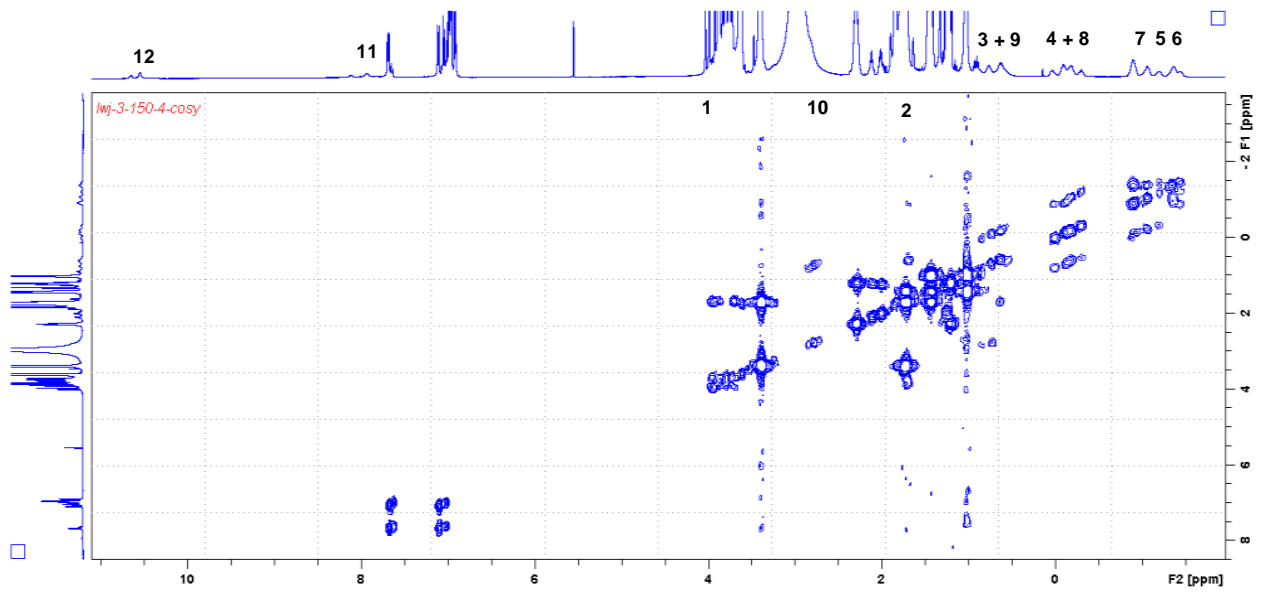

Figure S63: 2D ${ }^{1} \mathrm{H}-{ }^{1} \mathrm{H}$ COSY spectrum (THF- $d_{8}, 298 \mathrm{~K}, 500 \mathrm{MHz}$ ) of [c2]daisy chain rotaxane 2 with the addition of 6 eq. of TBAA. 


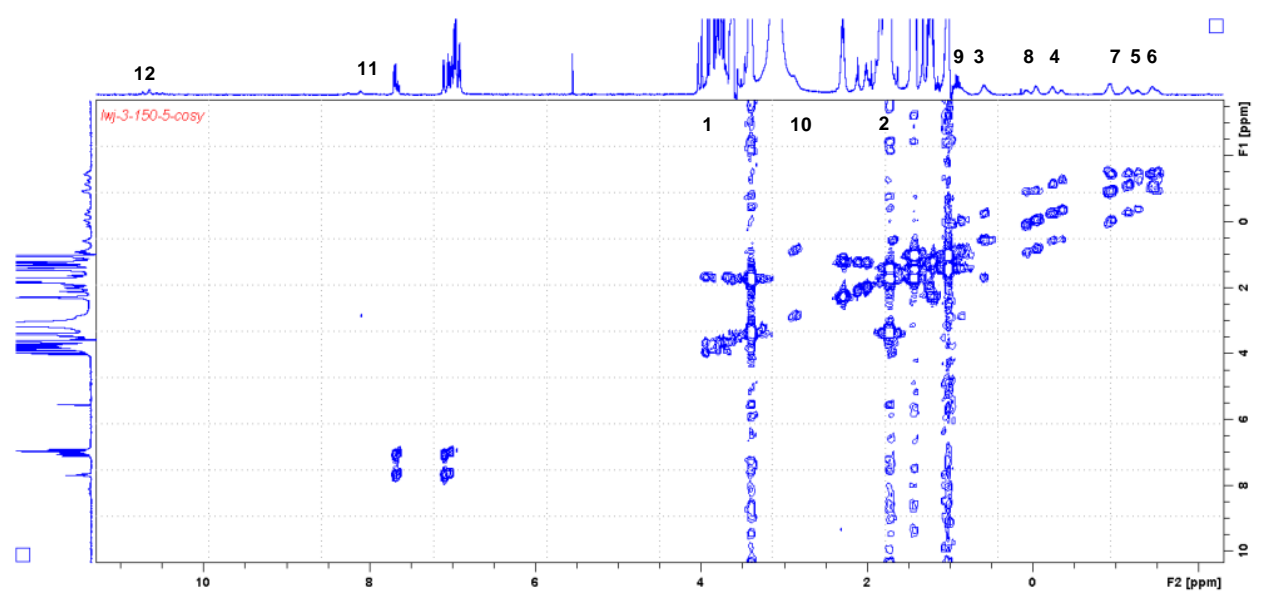

Figure S64: 2D ${ }^{1} \mathrm{H}-{ }^{1} \mathrm{H}$ COSY spectrum (THF- $d_{8}, 298 \mathrm{~K}, 500 \mathrm{MHz}$ ) of [c2]daisy chain rotaxane 2 with the addition of 8 eq. of TBAA.

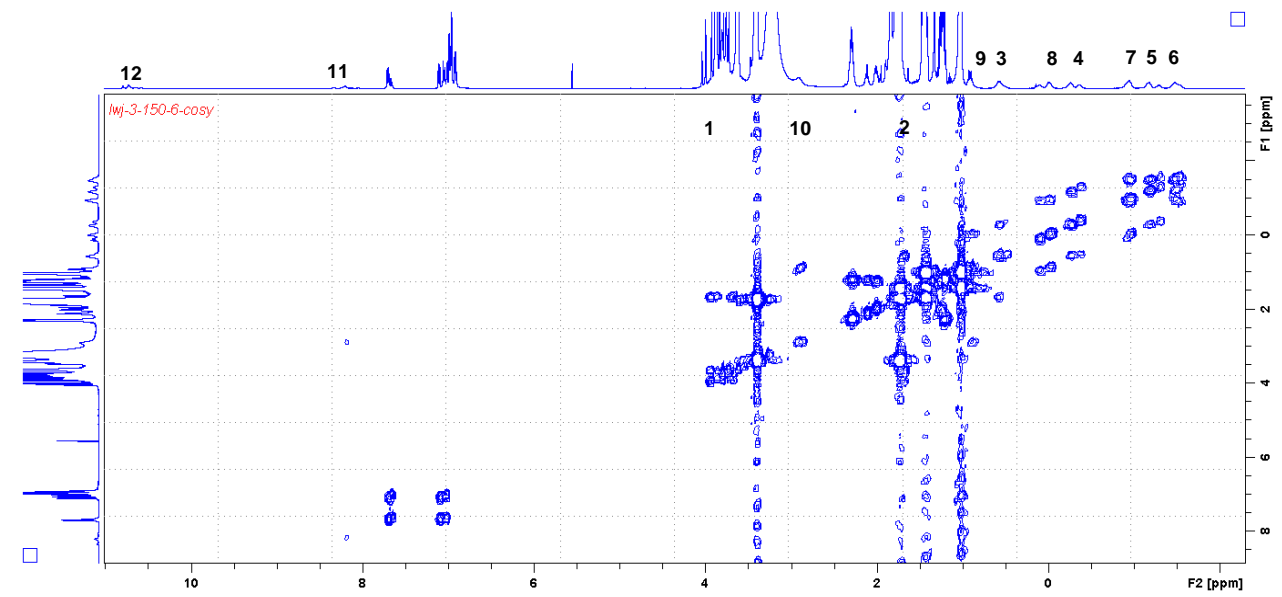

Figure S65: 2D ${ }^{1} \mathrm{H}-{ }^{1} \mathrm{H}$ COSY spectrum (THF- $d_{8}, 298 \mathrm{~K}, 500 \mathrm{MHz}$ ) of [c2]daisy chain rotaxane 2 with the addition of 10 eq. of TBAA.

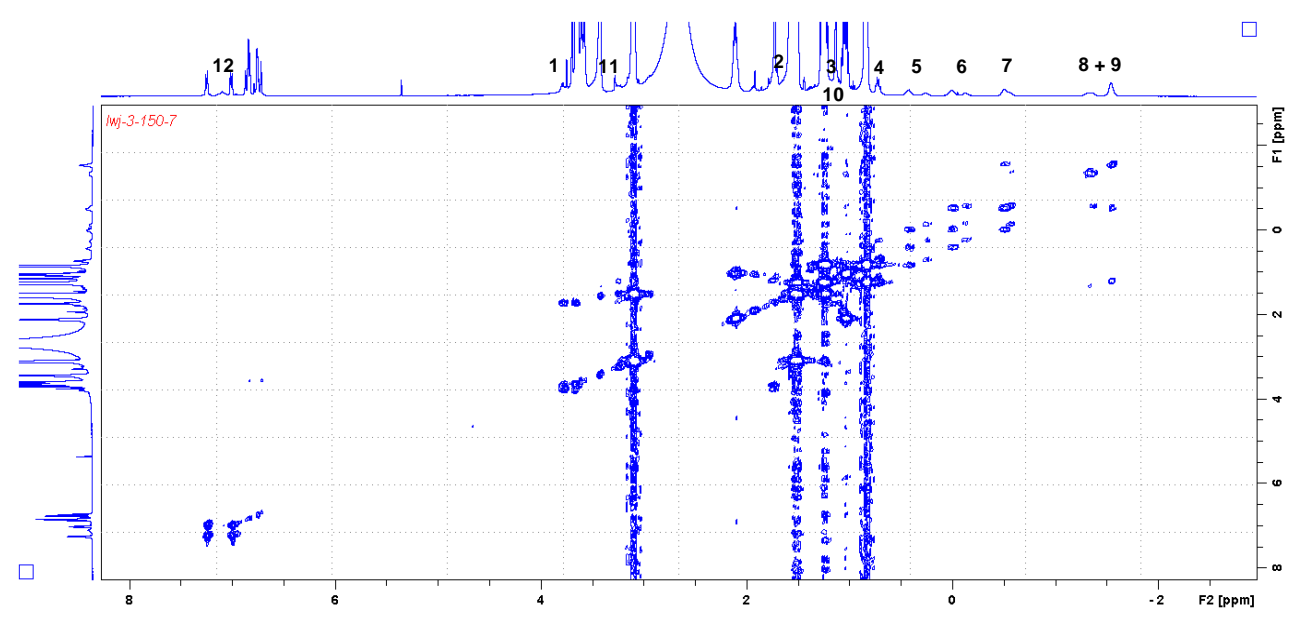

Figure S66: 2D ${ }^{1} \mathrm{H}-{ }^{1} \mathrm{H}$ COSY spectrum $\left(\mathrm{THF}-d_{8}, 298 \mathrm{~K}, 500 \mathrm{MHz}\right)$ of [c2]daisy chain rotaxane 2 with the addition of 10 eq. of $\mathrm{NaPF}_{6}$. 


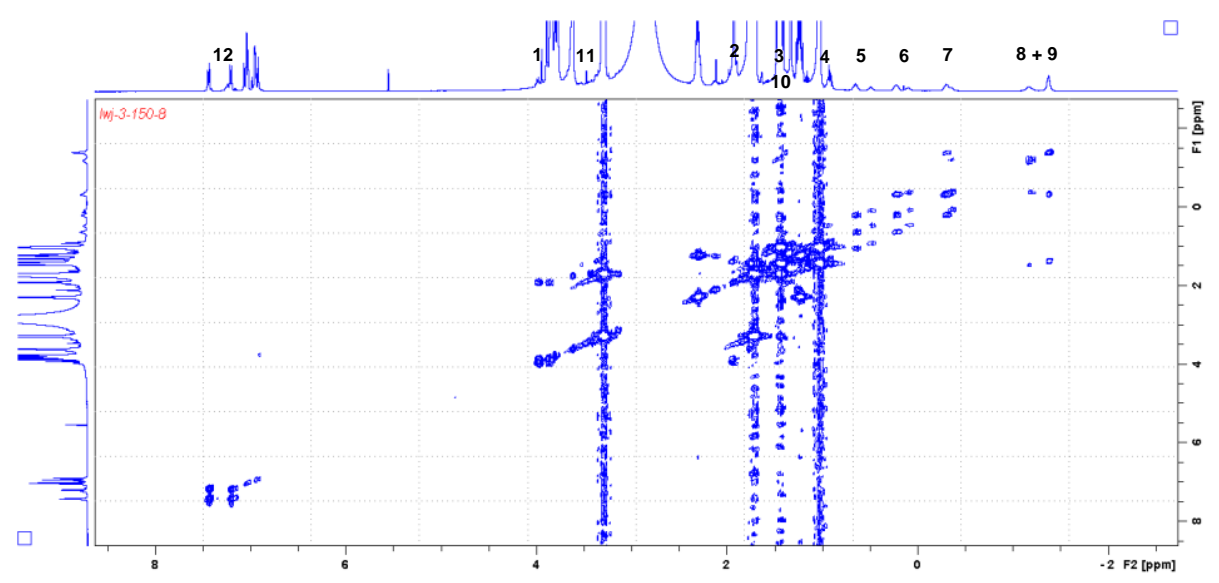

Figure S67: 2D ${ }^{1} \mathrm{H}_{-}{ }^{1} \mathrm{H}$ COSY spectrum (THF- $d_{8}, 298 \mathrm{~K}, 500 \mathrm{MHz}$ ) of [c2]daisy chain rotaxane 2 with the addition of 14 eq. of $\mathrm{NaPF}_{6}$.

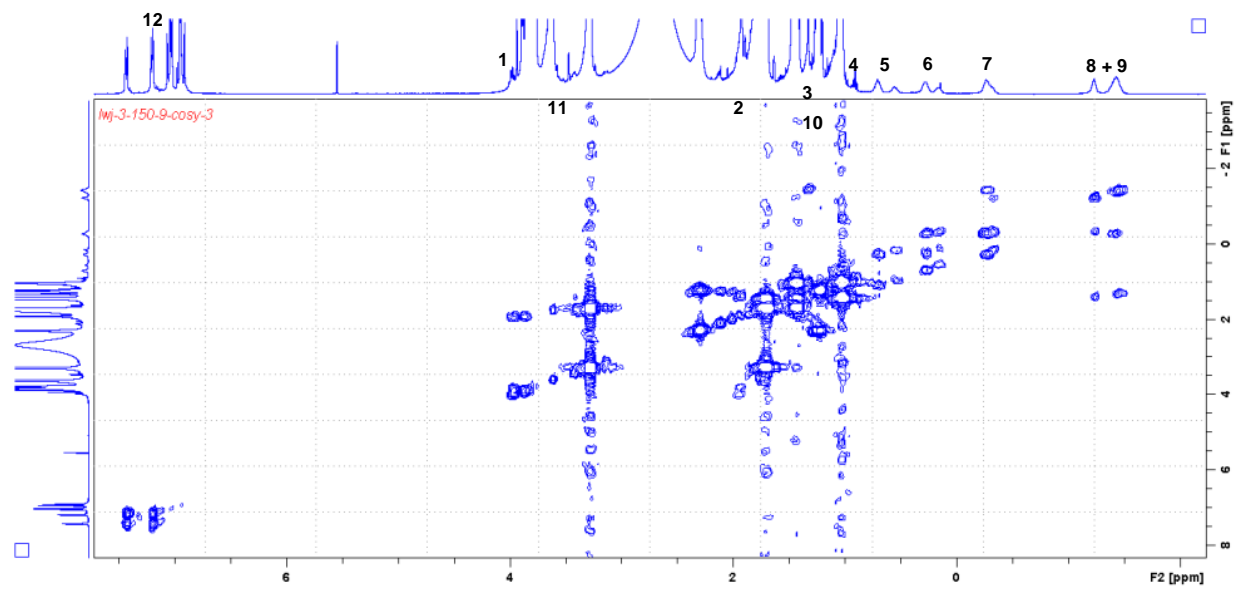

Figure S68: 2D ${ }^{1} \mathrm{H}-{ }^{1} \mathrm{H}$ COSY spectrum (THF- $d_{8}, 298 \mathrm{~K}, 500 \mathrm{MHz}$ ) of [c2]daisy chain rotaxane 2 with the addition of 16 eq. of $\mathrm{NaPF}_{6}$.

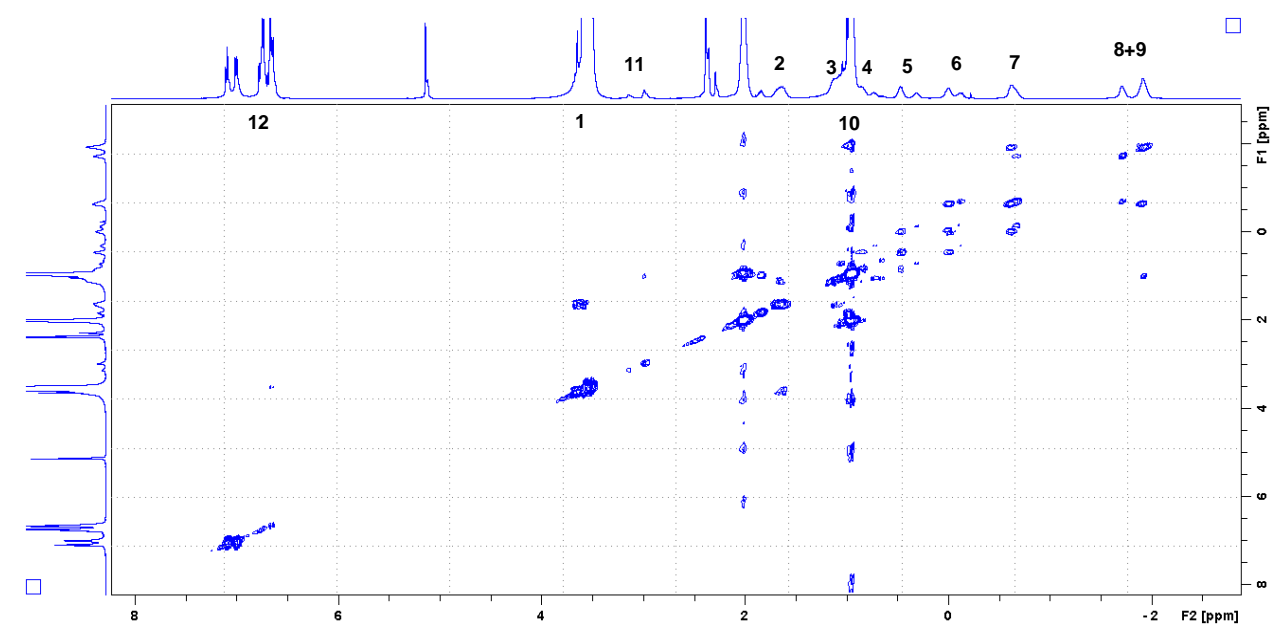

Figure S69: $2 \mathrm{D}{ }^{1} \mathrm{H}-{ }^{1} \mathrm{H}$ COSY spectrum $\left(\mathrm{CD}_{2} \mathrm{Cl}_{2}(400 \mu \mathrm{L}), 298 \mathrm{~K}, 500 \mathrm{MHz}\right)$ of [c2] daisy chain rotaxane 2 with the addition of $40 \mu \mathrm{L}$ DMSO- $d_{6}$. 


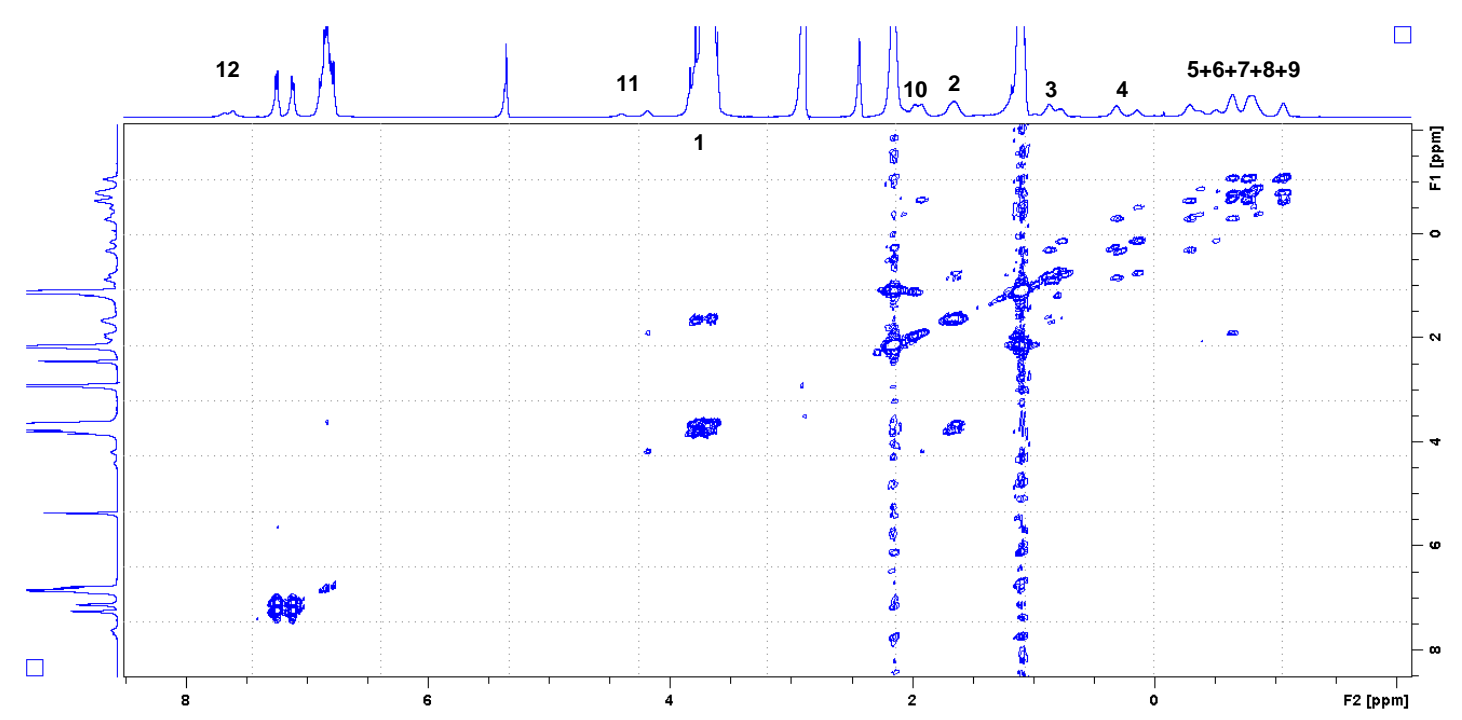

Figure S70: 2D ${ }^{1} \mathrm{H}-{ }^{1} \mathrm{H}$ COSY spectrum $\left(\mathrm{CD}_{2} \mathrm{Cl}_{2}(400 \mu \mathrm{L}), 298 \mathrm{~K}, 500 \mathrm{MHz}\right)$ of [c2]daisy chain rotaxane 2 with the addition of $120 \mu \mathrm{L}$ DMSO- $d_{6}$.

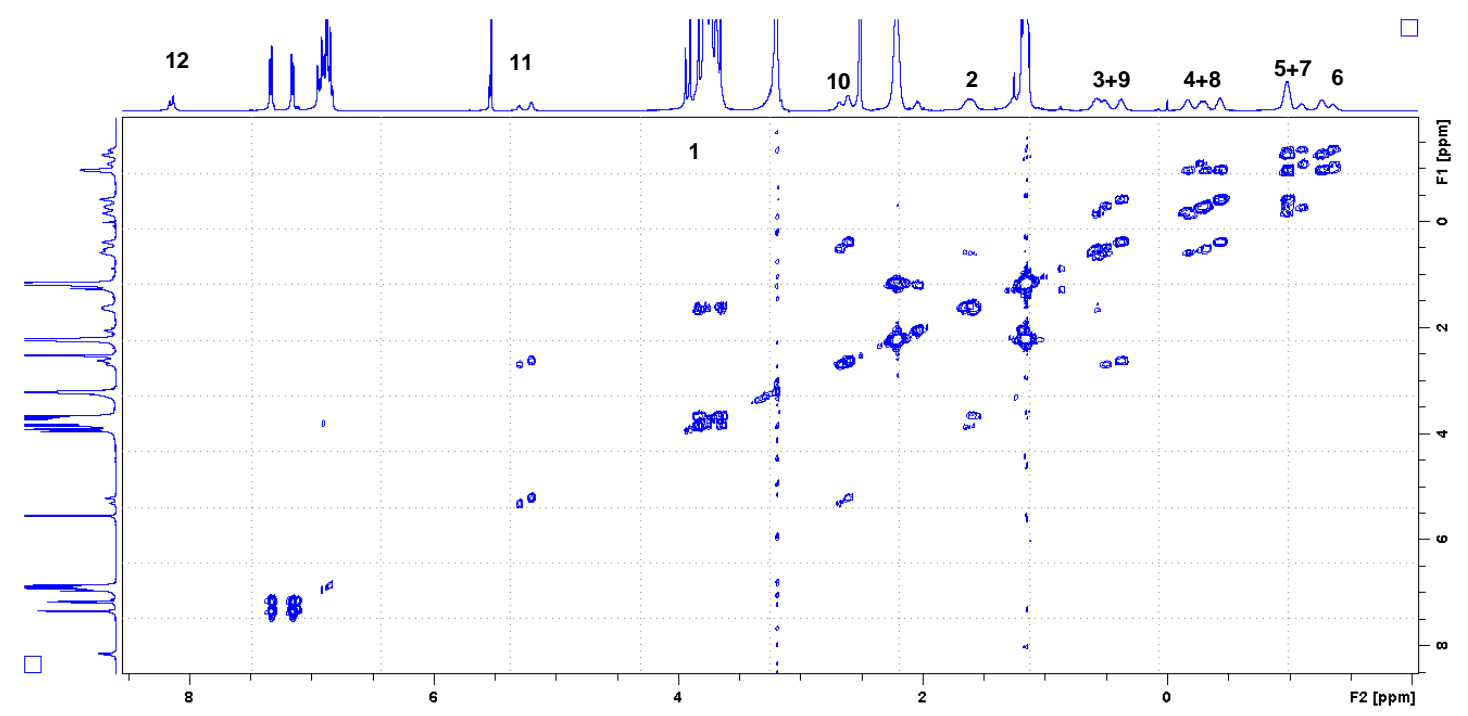

Figure S71: 2D ${ }^{1} \mathrm{H}-{ }^{1} \mathrm{H}$ COSY spectrum $\left(\mathrm{CD}_{2} \mathrm{Cl}_{2}(400 \mu \mathrm{L}), 298 \mathrm{~K}, 500 \mathrm{MHz}\right)$ of [c2]daisy chain rotaxane 2 with the addition of $320 \mu \mathrm{L}$ DMSO- $d_{6}$. 


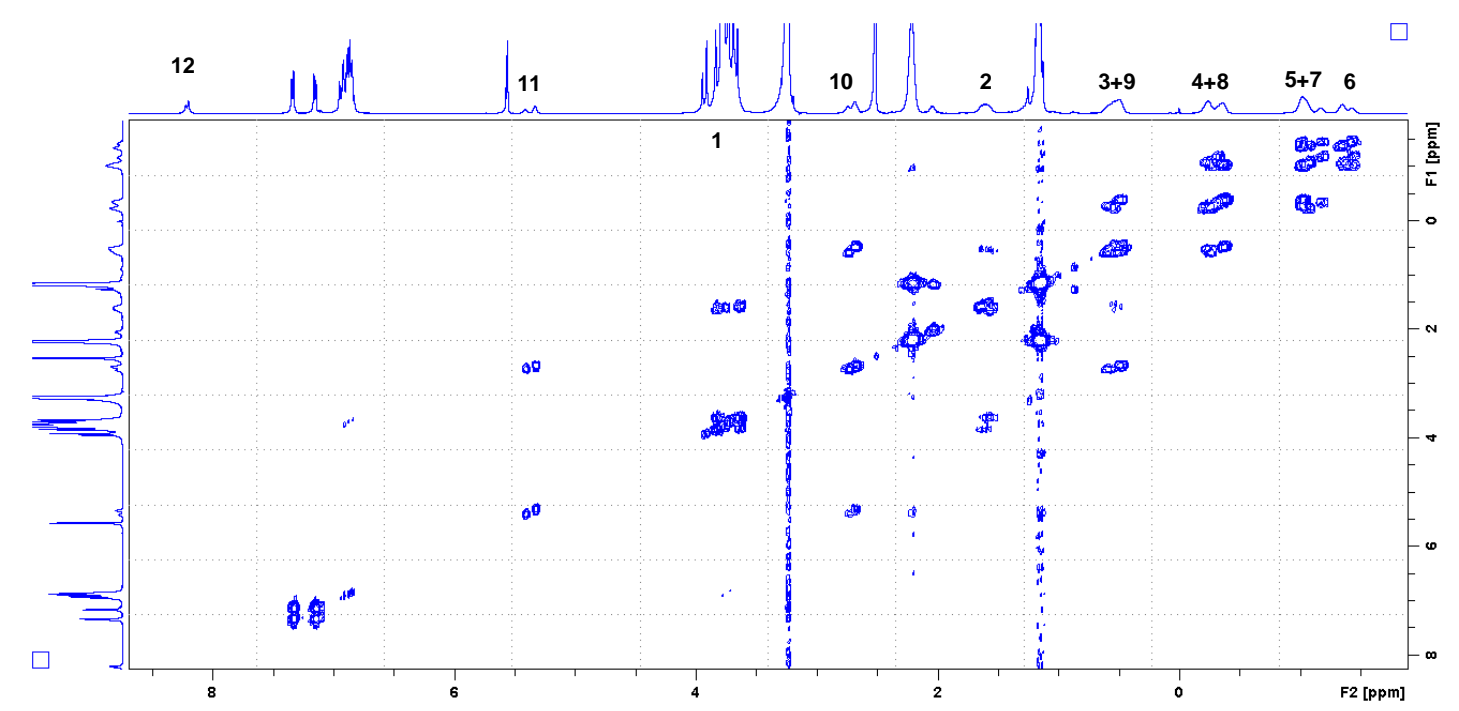

Figure S72: 2D ${ }^{1} \mathrm{H}-{ }^{1} \mathrm{H}$ COSY spectrum $\left(\mathrm{CD}_{2} \mathrm{Cl}_{2}(400 \mu \mathrm{L}), 298 \mathrm{~K}, 500 \mathrm{MHz}\right)$ of [c2]daisy chain rotaxane 2 with the addition of $400 \mu \mathrm{L}$ DMSO- $d_{6}$.

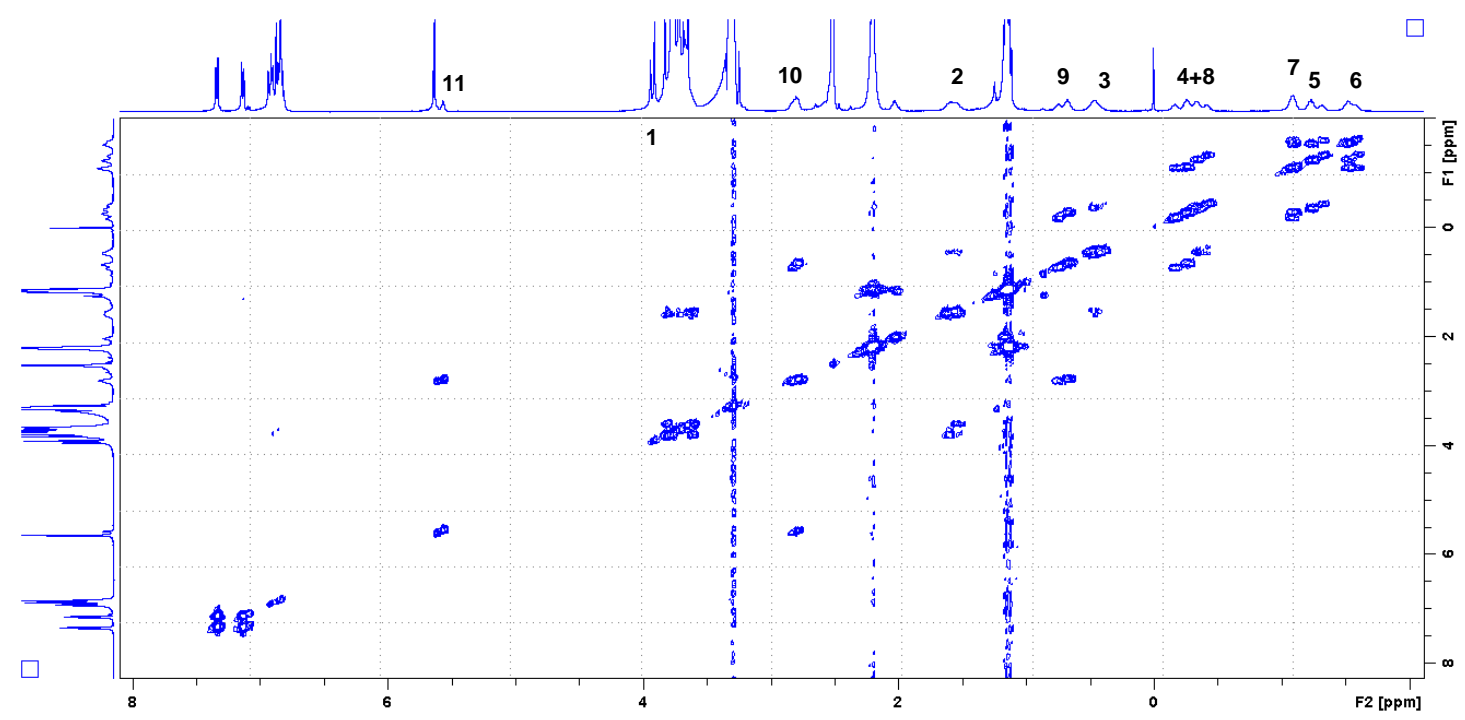

Figure S73: 2D ${ }^{1} \mathrm{H}-{ }^{1} \mathrm{H}$ COSY spectrum $\left(\mathrm{CD}_{2} \mathrm{Cl}_{2}(400 \mu \mathrm{L}), 298 \mathrm{~K}, 500 \mathrm{MHz}\right)$ of [c2]daisy chain rotaxane 2 with the addition of $800 \mu \mathrm{L}$ DMSO- $d_{6}$. 
(a)

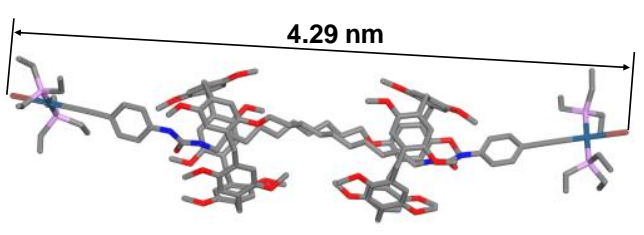

(c)

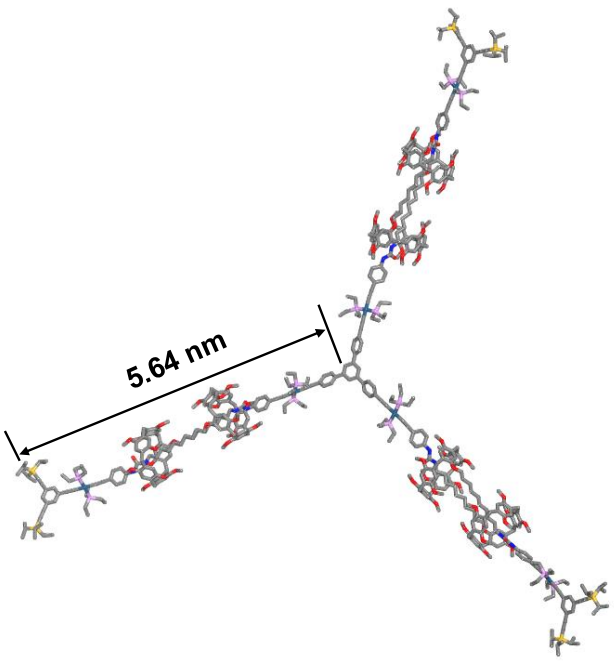

(b)

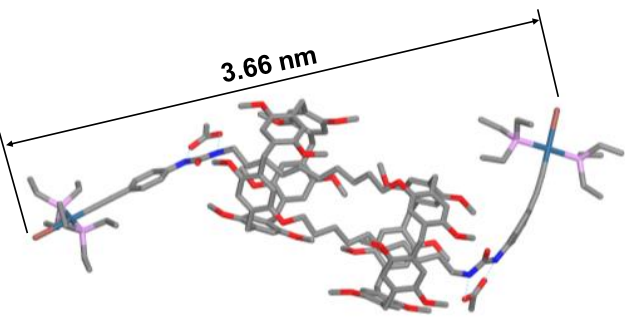

(d)

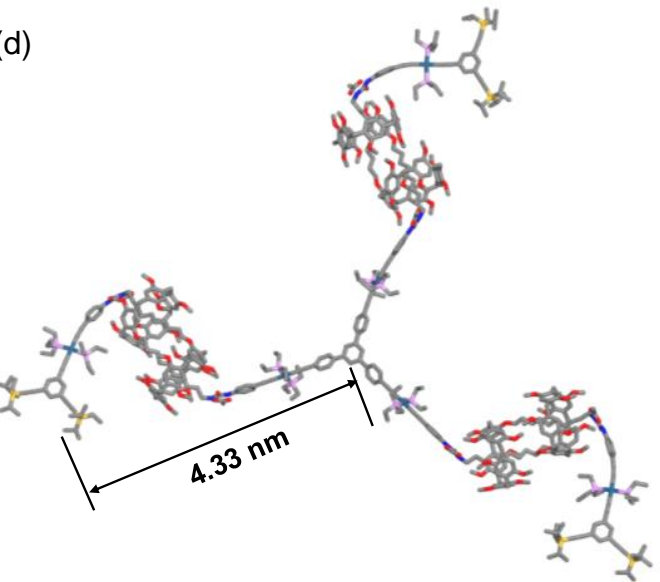

Figure S74: Theoretically calculated structures of (a) [c2]daisy chain rotaxane 2; (b) folded [c2] daisy chain rotaxane 2 upon binding with acetate anion; (c) DC-G1; (d) folded DC-G1 upon binding with acetate anion. (The monomer 2 with and without acetate anions was optimized at a semi-empirical method PM7 using the program MOPAC2016. PM7 optimized structures were further optimized at BP86/def2-SVP level with Grimme's dispersion correction by TURBOMOLE 7.1 program packages ${ }^{\mathrm{S} 5-\mathrm{S} 8}$. Unfortunately, the optimization of the monomer 2 with the inclusion of acetate anions did not converge at the DFT level. We only obtained the DFT optimized monomer without the anions. The first-generation daisy chain dendrimer DC-G1 was optimized by the semi-empirical quantum mechanical method GFN2-xTB5 in the xtb code in version 6.2.2 ${ }^{\mathrm{S} 9}$.) 
Section 5. Anion- and solvent-induced dimension modulation of the daisy chain dendrimers

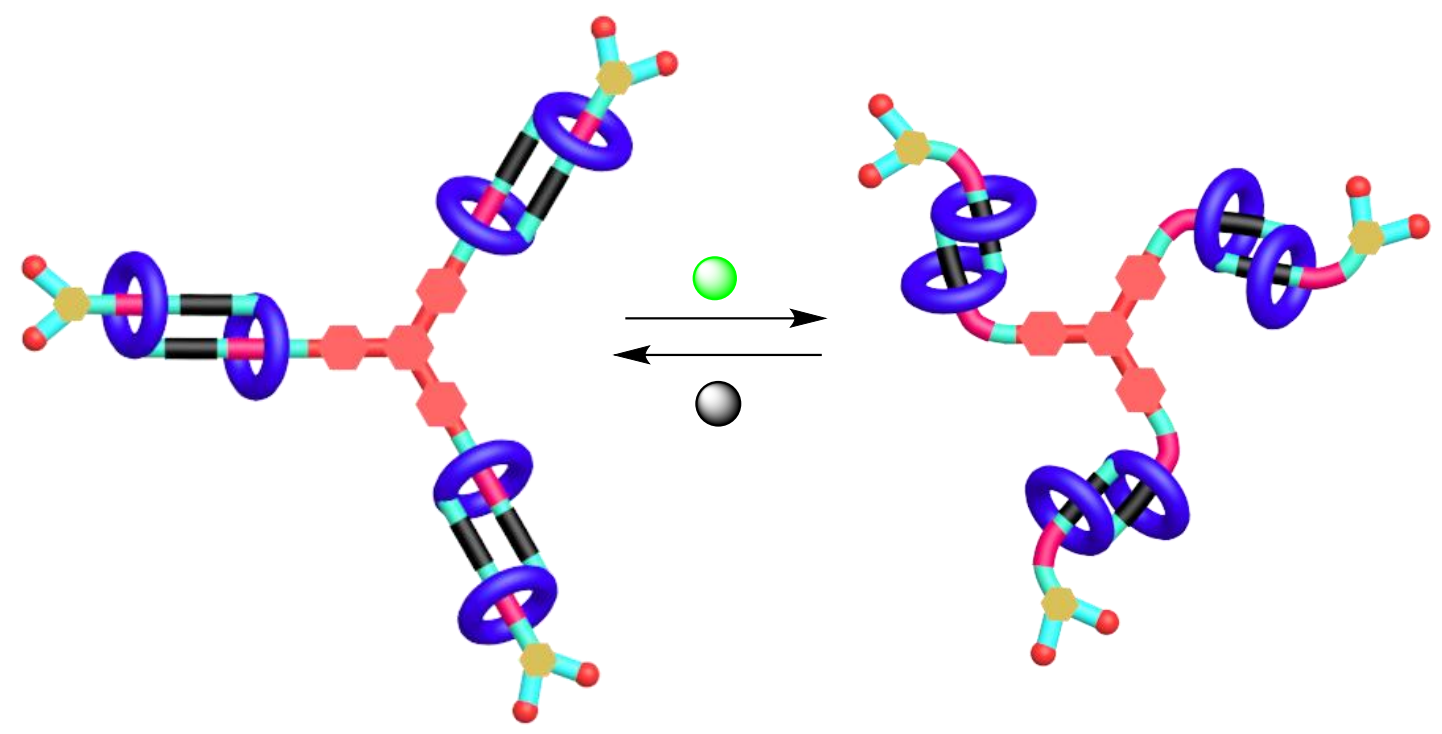

Figure S75: Anion- and solvent-induced dimension modulation of the daisy chain dendrimer DC-G1.

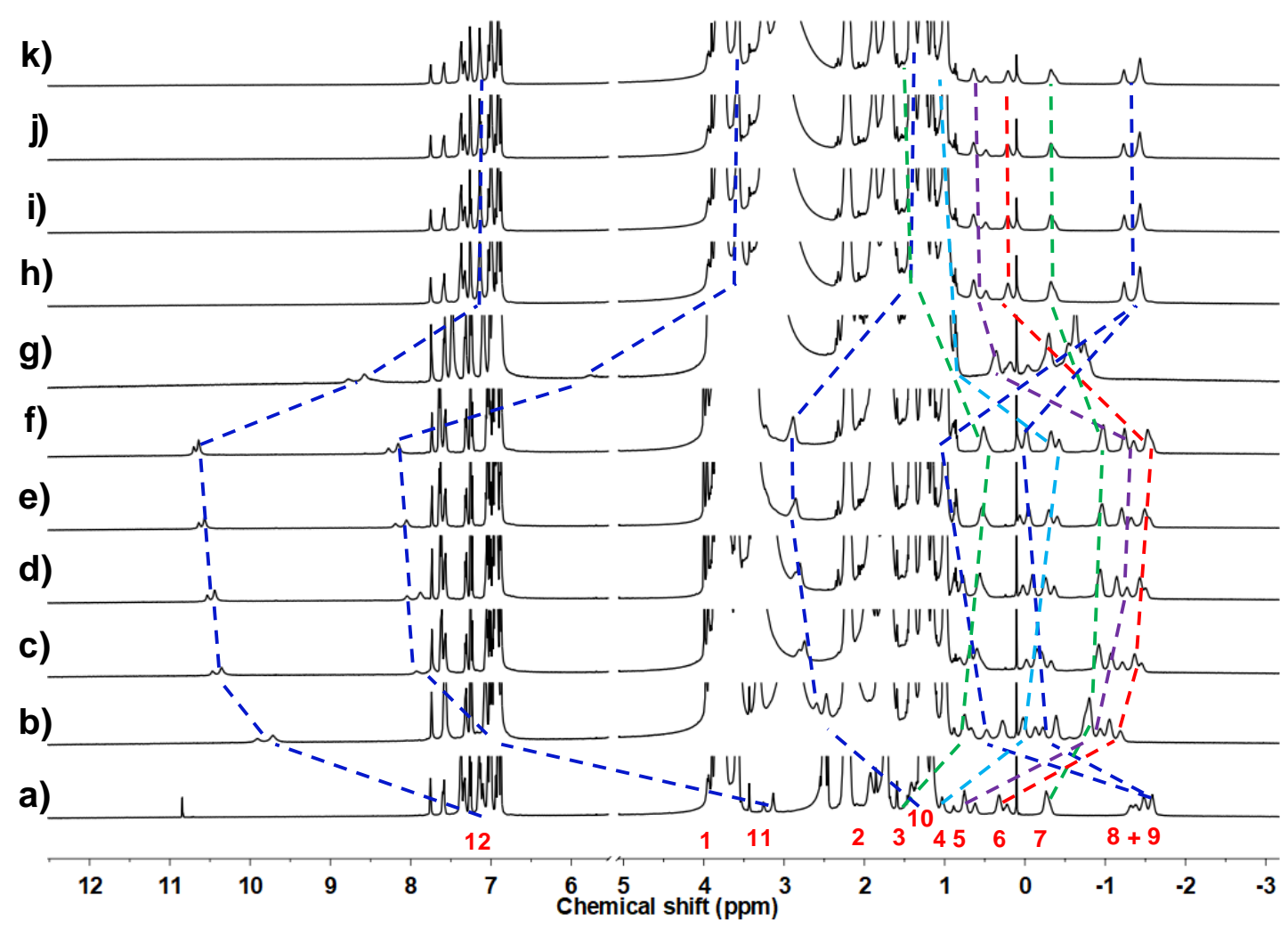

Figure S76: ${ }^{1} \mathrm{H}$ NMR spectra (THF- $d_{8}, 298 \mathrm{~K}, 500 \mathrm{MHz}$ ) of anion-induced switching motion of the daisy chain dendrimer DC-G1. a) DC-G1; the mixture of DC-G1 and 
TBAA, for each [c2]daisy chain unit: b) TBAA (2 equiv); (c) TBAA (4 equiv); d) TBAA (6 equiv); e) TBAA ( 8 equiv); f) TBAA (10 equiv); and the mixture obtained after adding $\mathrm{NaPF}_{6}$ to the solution in $\mathrm{f}$ ): $\left.\mathrm{g}\right) \mathrm{NaPF}_{6}$ (10 equiv); $\left.\mathrm{h}\right) \mathrm{NaPF}_{6}$ (14 equiv); i) $\mathrm{NaPF}_{6}$ (16 equiv); j) $\mathrm{NaPF}_{6}$ (20 equiv); k) $\mathrm{NaPF}_{6}$ (26 equiv).

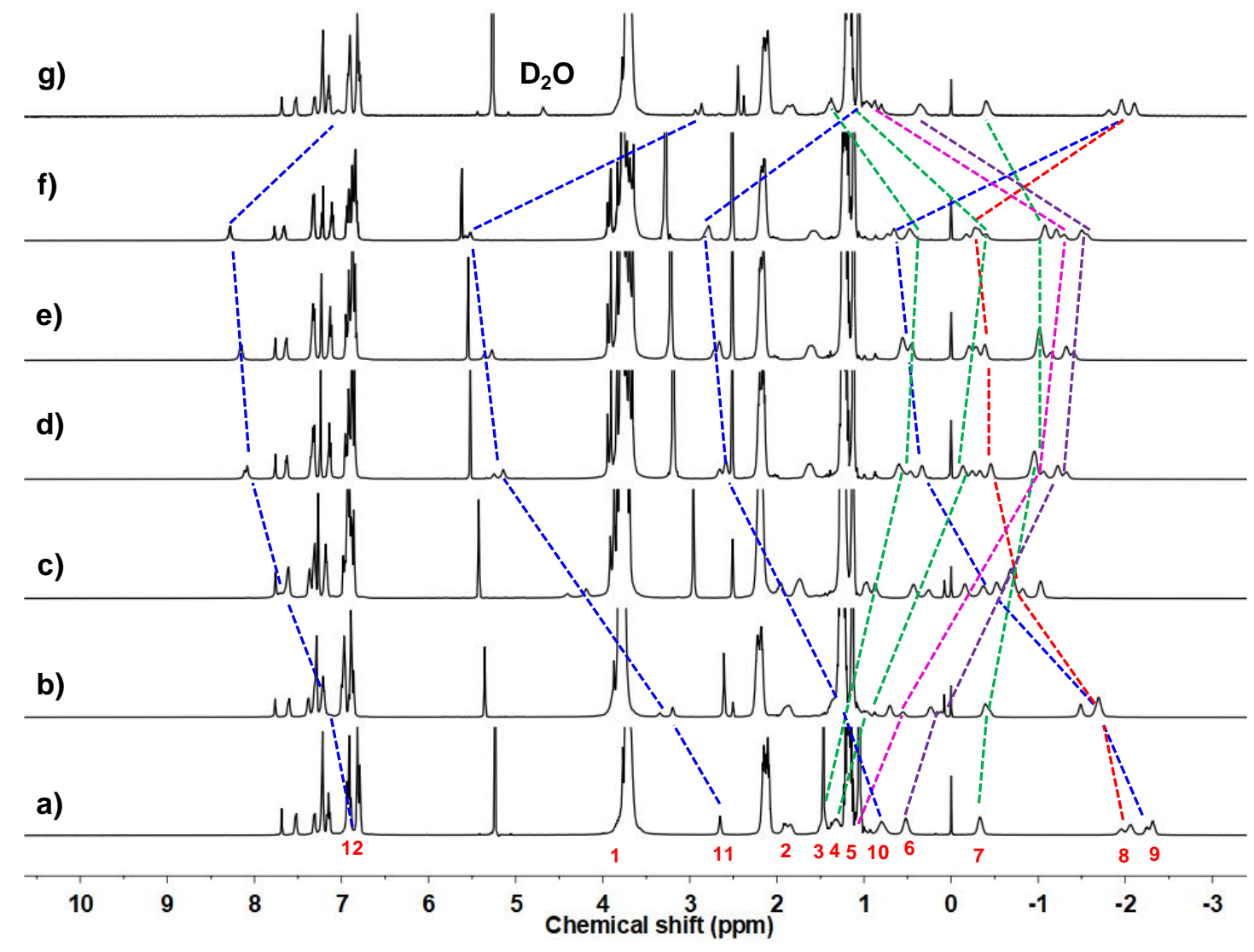

Figure S77: ${ }^{1} \mathrm{H}$ NMR spectra $\left(\mathrm{CD}_{2} \mathrm{Cl}_{2}, 298 \mathrm{~K}, 500 \mathrm{MHz}\right)$ of solvent-induced dimension modulation of the daisy chain dendrimer DC-G1. a) DC-G1 in $\mathrm{CD}_{2} \mathrm{Cl}_{2}$ (400 $\mu \mathrm{L})$; the addition of DMSO- $d_{6}$ in a): b) $40 \mu \mathrm{L}$; c) $120 \mu \mathrm{L}$; d) $320 \mu \mathrm{L}$; e) $400 \mu \mathrm{L}$; f) $800 \mu \mathrm{L} ; \mathrm{g})$ the addition of $\mathrm{D}_{2} \mathrm{O}(800 \mu \mathrm{L})$ in $\left.\mathrm{f}\right)$. 


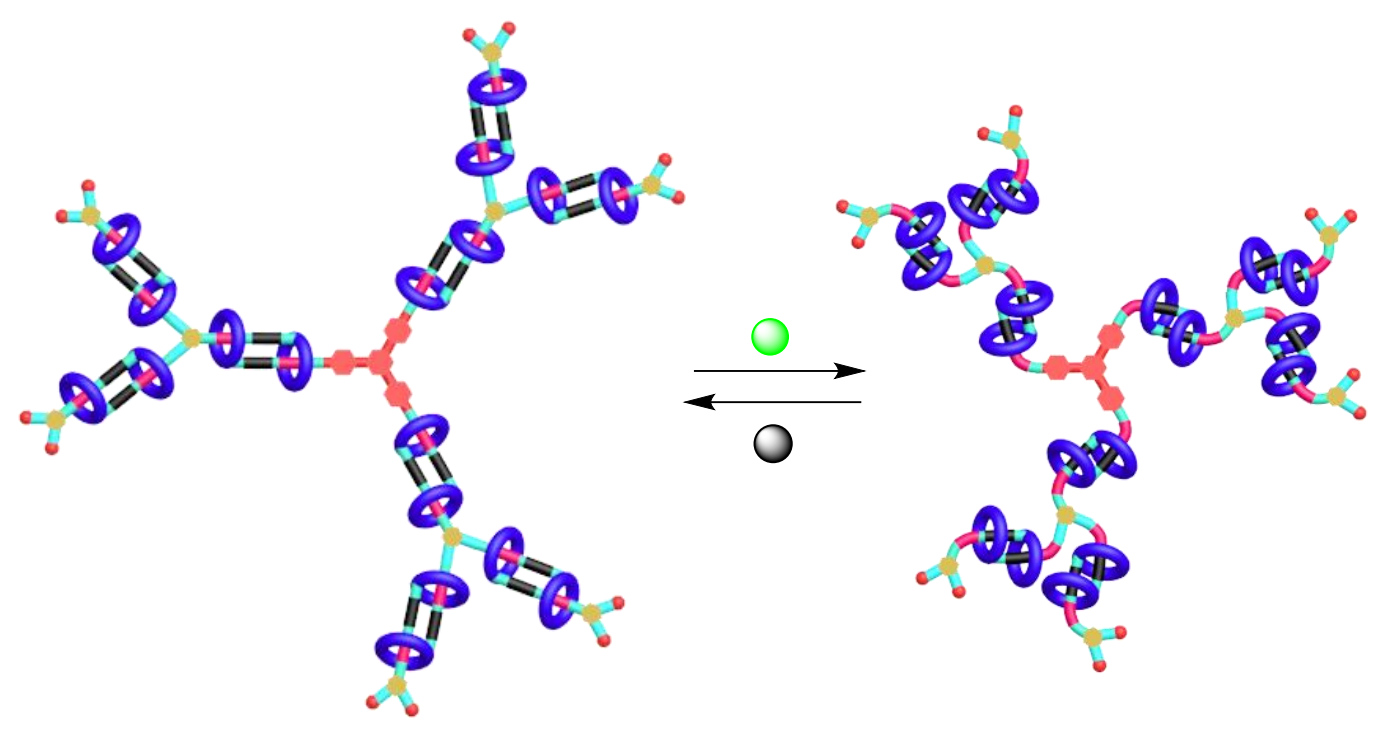

Figure 78: Anion- and solvent-induced dimension modulation of daisy chain dendrimer DC-G2.

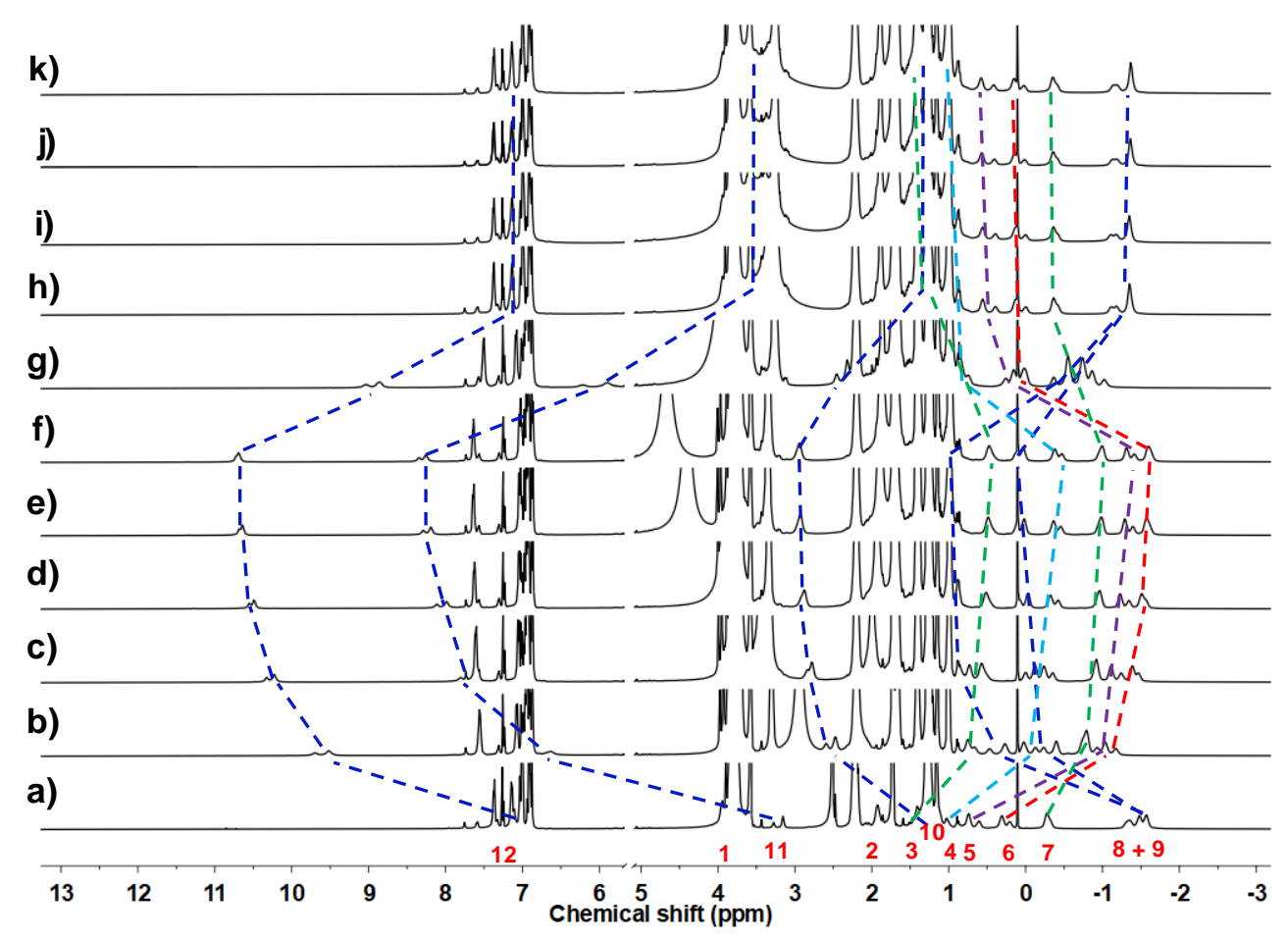

Figure S79: ${ }^{1} \mathrm{H}$ NMR spectra (THF- $d_{8}, 298 \mathrm{~K}, 500 \mathrm{MHz}$ ) of anion-induced dimension modulation of the daisy chain dendrimer DC-G2. a) DC-G2; the mixture of DC-G2 and TBAA, for each [c2]daisy chain unit: b) TBAA (2 equiv); (c) TBAA (4 equiv); d) TBAA (6 equiv); e) TBAA ( 8 equiv); f) TBAA (10 equiv); and the mixture obtained after adding $\mathrm{NaPF}_{6}$ to the solution in $\left.\left.\mathrm{f}\right): \mathrm{g}\right) \mathrm{NaPF}_{6}$ (10 equiv); h) $\mathrm{NaPF}_{6}$ (14 equiv); i) $\mathrm{NaPF}_{6}$ (16 equiv); j) $\mathrm{NaPF}_{6}(20$ equiv); $\mathrm{k}) \mathrm{NaPF}_{6}$ (30 equiv). 


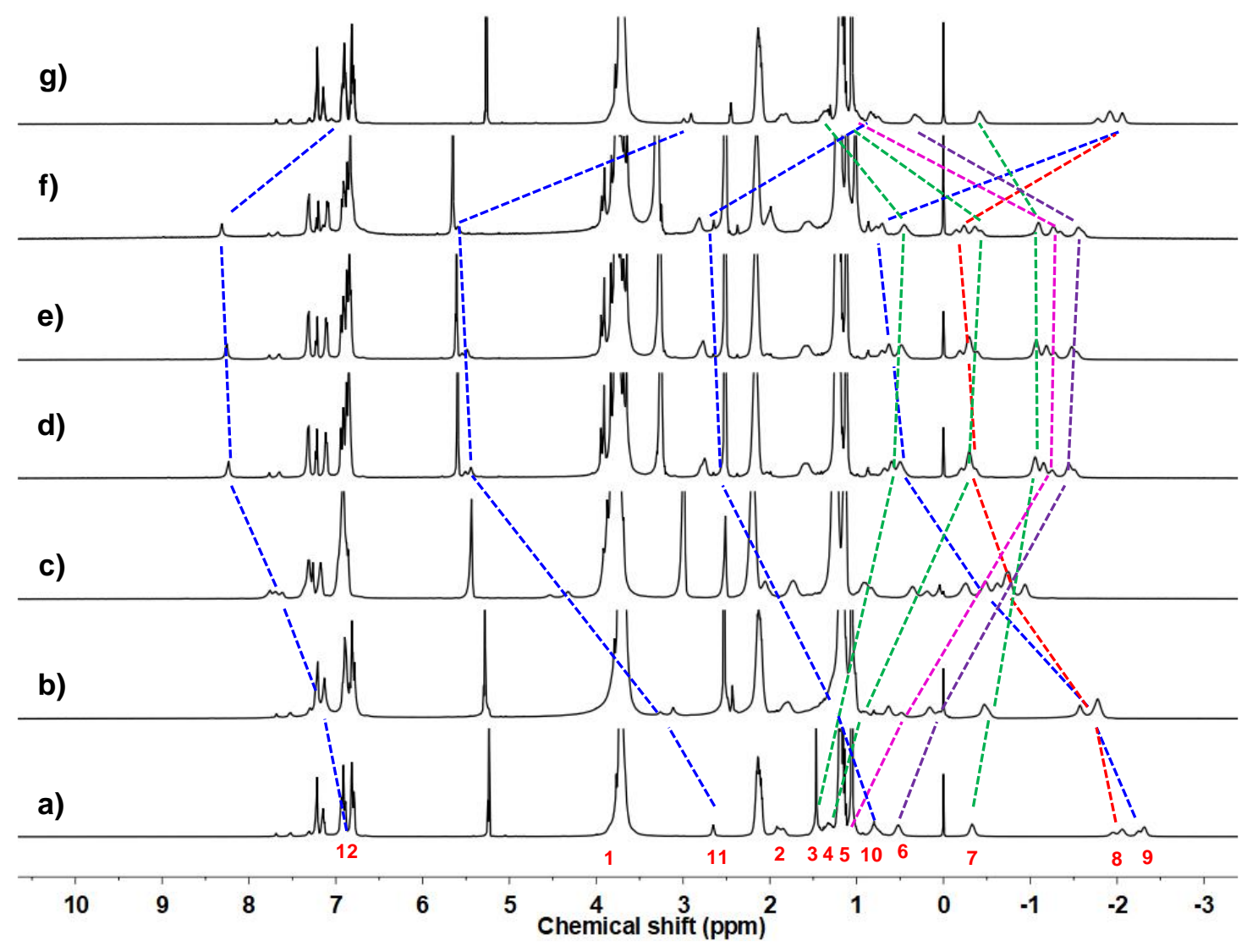

Figure S80: ${ }^{1} \mathrm{H}$ NMR spectra $\left(\mathrm{CD}_{2} \mathrm{Cl}_{2}, 298 \mathrm{~K}, 500 \mathrm{MHz}\right)$ of solvent-induced dimension modulation of the daisy chain dendrimer DC-G2. a) DC-G2 in $\mathrm{CD}_{2} \mathrm{Cl}_{2}$ ( $400 \mu \mathrm{L})$; the addition of DMSO- $d_{6}$ in a): b) $40 \mu \mathrm{L}$; c) $120 \mu \mathrm{L}$; d) $320 \mu \mathrm{L}$; e) $400 \mu \mathrm{L}$; f) $800 \mu \mathrm{L} ; \mathrm{g})$ the addition of $\mathrm{D}_{2} \mathrm{O}(800 \mu \mathrm{L})$ in $\left.\mathrm{f}\right)$.

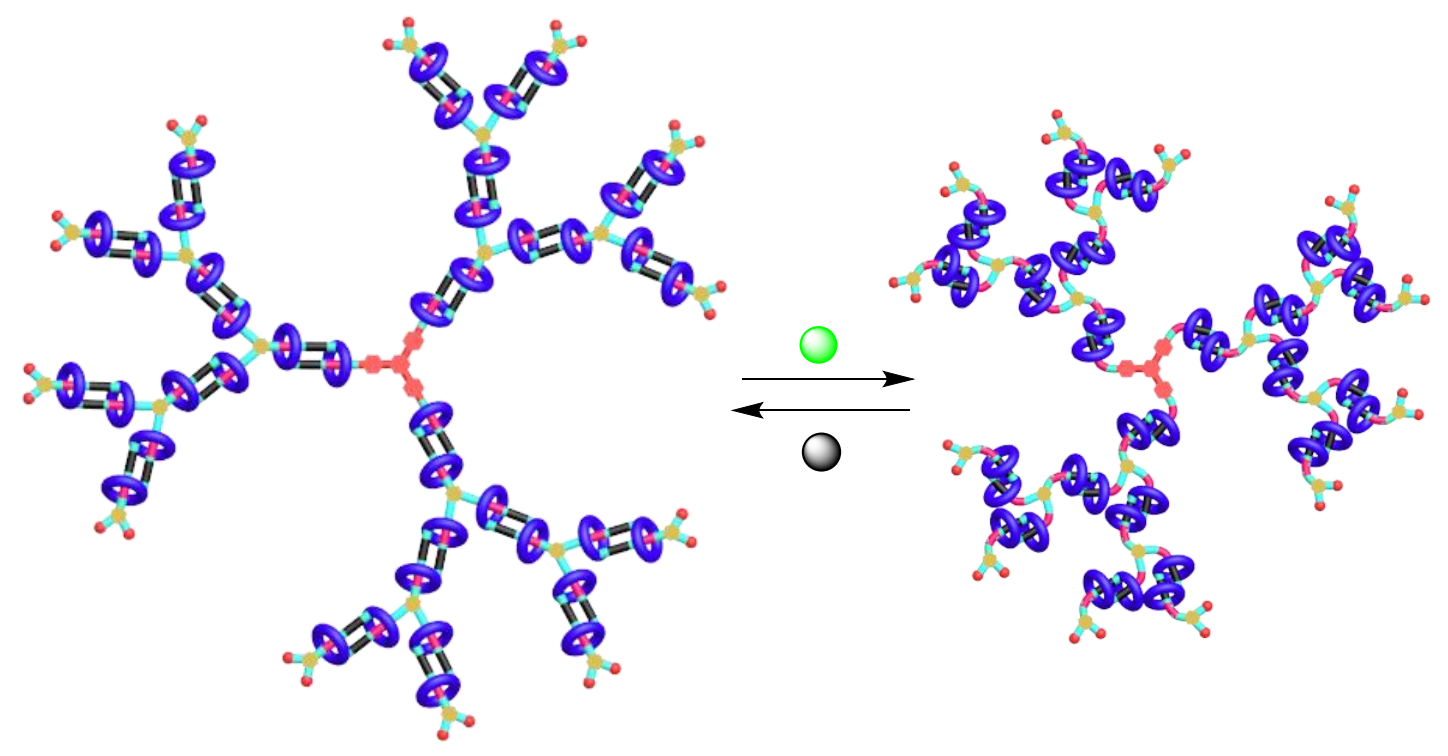

Figure S81: Anion- and solvent-induced dimension modulation of daisy chain dendrimer DC-G3. 


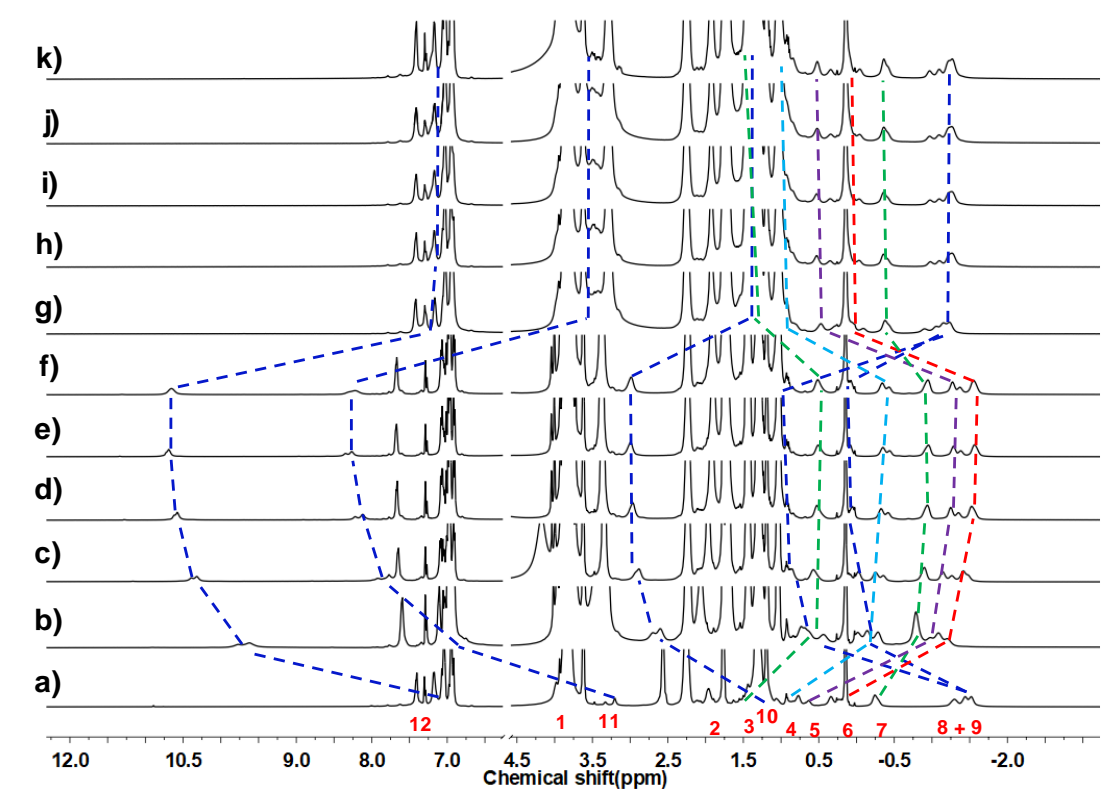

Figure S82: ${ }^{1} \mathrm{H}$ NMR spectra (THF- $d_{8}, 298 \mathrm{~K}, 500 \mathrm{MHz}$ ) of anion-induced dimension modulation of the daisy chain dendrimer DC-G3. a) DC-G3; the mixture of DC-G3 and TBAA, for each [c2]daisy chain unit: b) TBAA (2 equiv); (c) TBAA (4 equiv); d) TBAA (6 equiv); e) TBAA ( 8 equiv); f) TBAA (10 equiv); and the mixture obtained after adding $\mathrm{NaPF}_{6}$ to the solution in $\mathrm{f}$ ): $\left.\mathrm{g}\right) \mathrm{NaPF}_{6}$ (10 equiv); h) $\mathrm{NaPF}_{6}$ (14 equiv); i) $\mathrm{NaPF}_{6}$ (16 equiv); $\left.\mathrm{j}\right) \mathrm{NaPF}_{6}$ (20 equiv); $\left.\mathrm{k}\right) \mathrm{NaPF}_{6}$ (30 equiv).

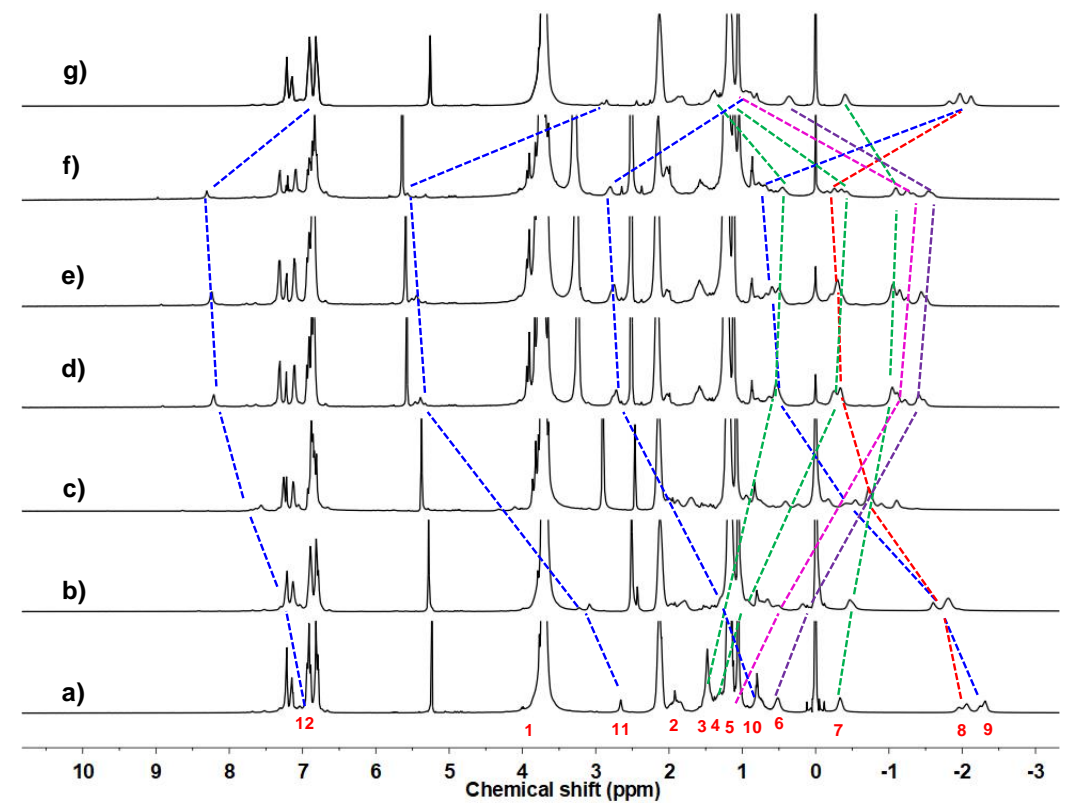

Figure S83: ${ }^{1} \mathrm{H}$ NMR spectra $\left(\mathrm{CD}_{2} \mathrm{Cl}_{2}, 298 \mathrm{~K}, 500 \mathrm{MHz}\right)$ of solvent-induced dimension modulation of the daisy chain dendrimer DC-G3. a) DC-G3 in $\mathrm{CD}_{2} \mathrm{Cl}_{2}$ $(400 \mu \mathrm{L})$; the addition of DMSO- $d_{6}$ in a): b) $40 \mu \mathrm{L}$; c) $120 \mu \mathrm{L}$; d) $320 \mu \mathrm{L}$; e) $400 \mu \mathrm{L}$; f) $800 \mu \mathrm{L} ; \mathrm{g})$ the addition of $\mathrm{D}_{2} \mathrm{O}(800 \mu \mathrm{L})$ in $\left.\mathrm{f}\right)$. 


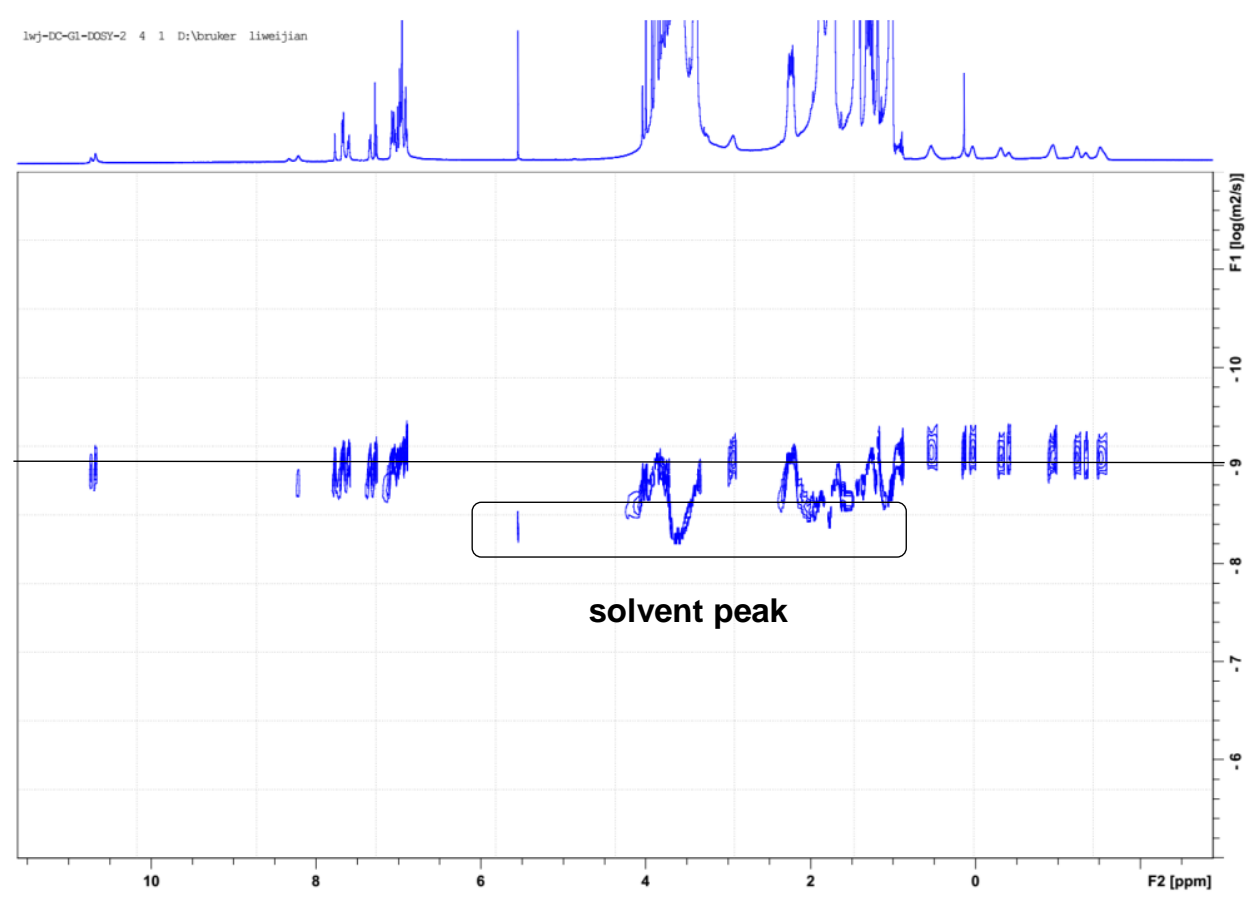

Figure S84: 2-D DOSY spectrum (THF- $d_{8}, 298 \mathrm{~K}, 500 \mathrm{MHz}$ ) of the daisy chain dendrimer DC-G1 with the addition of TBAA (10 equiv. for each [c2]daisy chain unit).

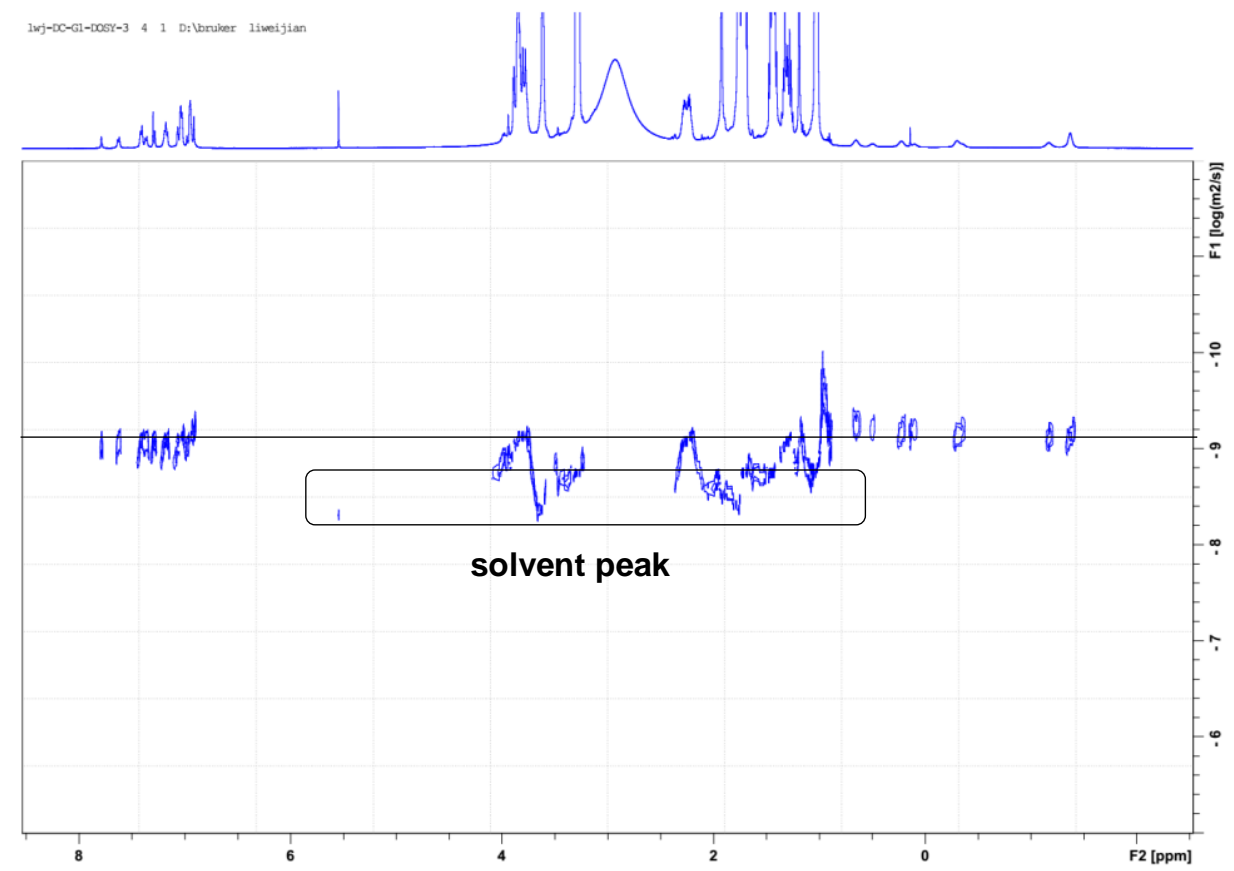

Figure S85: 2-D DOSY spectrum (THF- $\left.d_{8}, 298 \mathrm{~K}, 500 \mathrm{MHz}\right)$ of the mixture after adding $\mathrm{NaPF}_{6}$ into the solution of the daisy chain dendrimer DC-G1 and TBAA. 


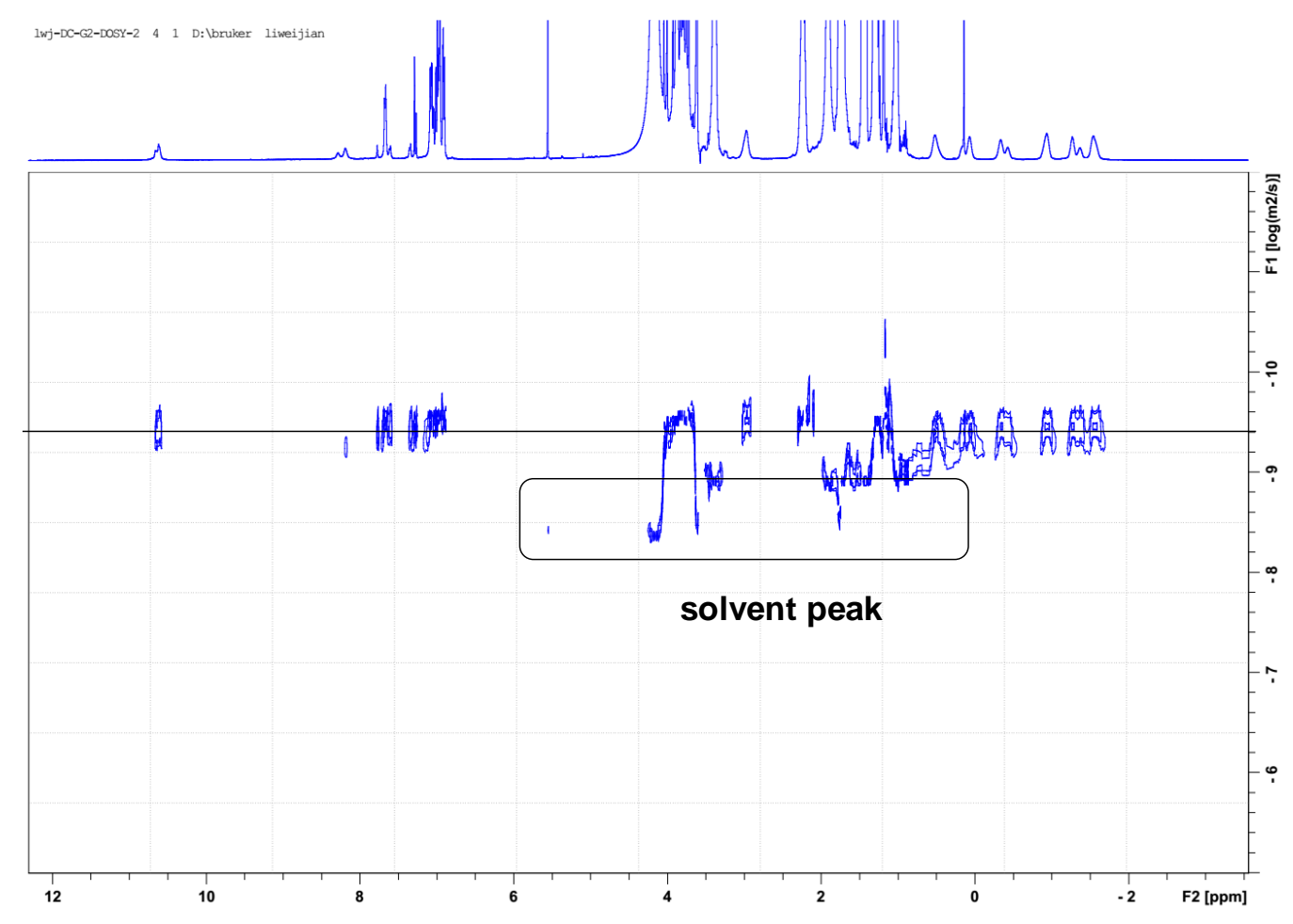

Figure S86: 2-D DOSY spectrum (THF- $d_{8}, 298 \mathrm{~K}, 500 \mathrm{MHz}$ ) of the daisy chain dendrimer DC-G2 with the addition of TBAA (10 equiv. for each [c2]daisy chain unit).

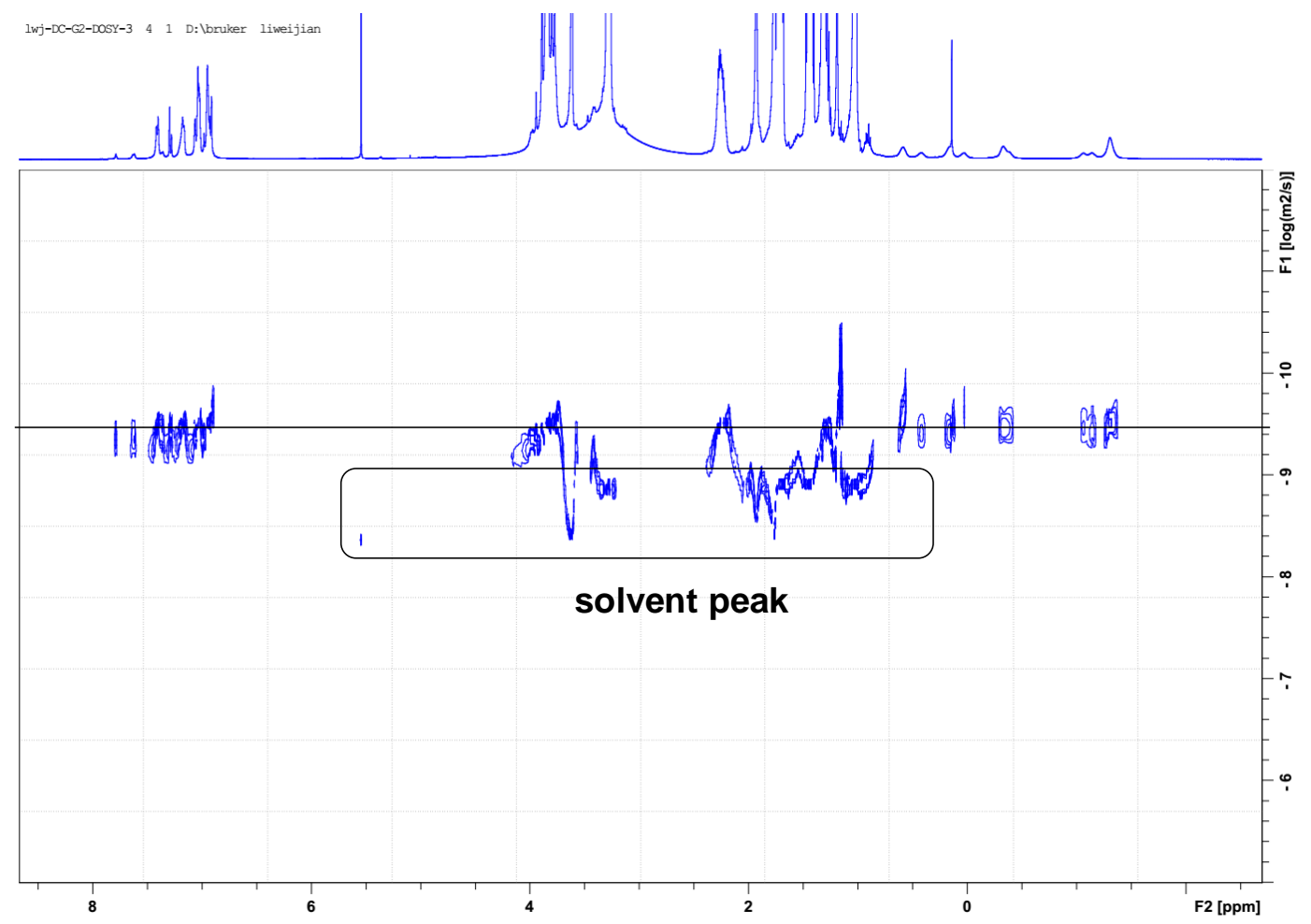

Figure S87: 2-D DOSY spectrum (THF- $d_{8}, 298 \mathrm{~K}, 500 \mathrm{MHz}$ ) of the mixture after adding $\mathrm{NaPF}_{6}$ into the solution of the daisy chain dendrimer DC-G2 and TBAA. 


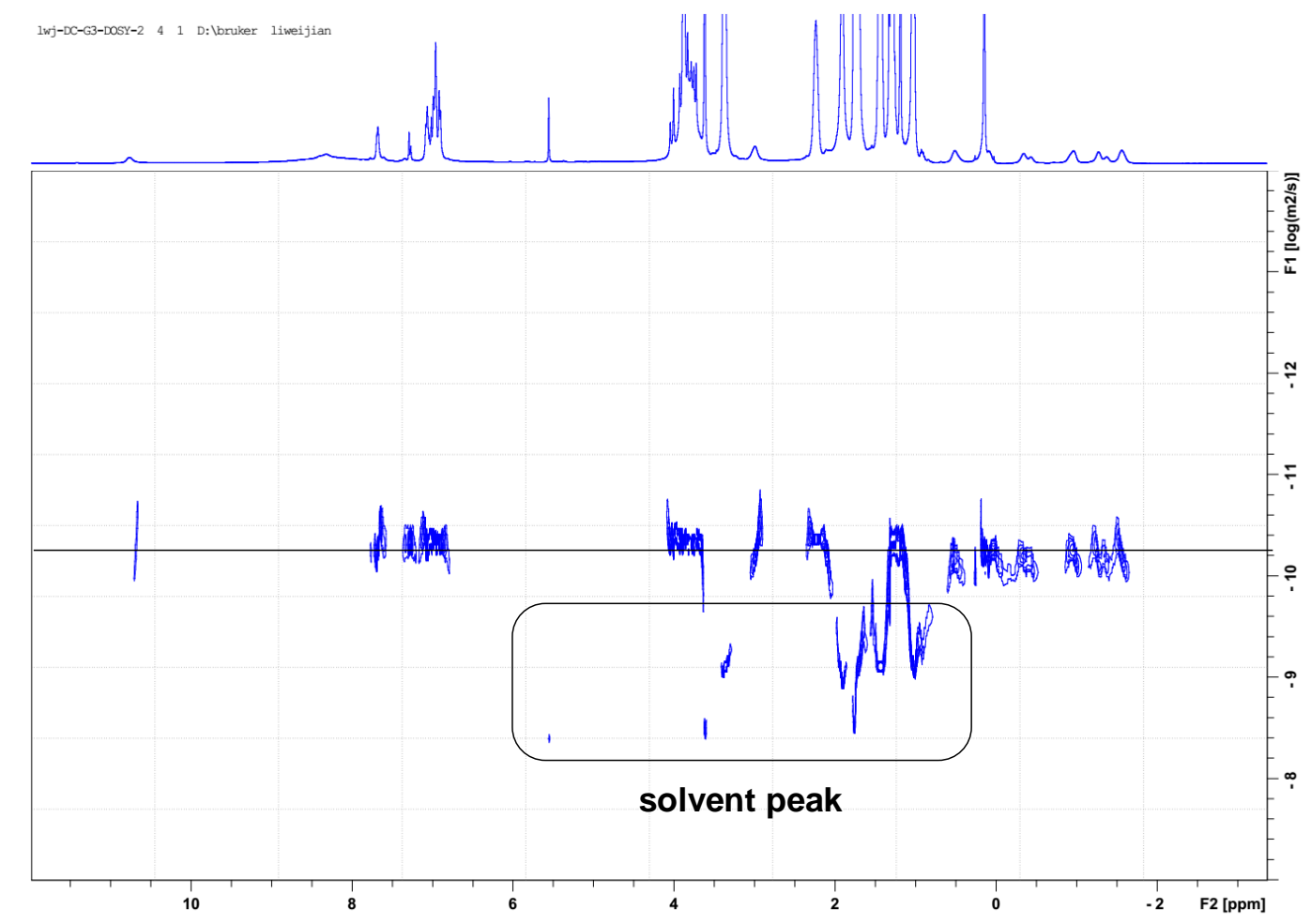

Figure S88: 2-D DOSY spectrum (THF- $d_{8}, 298 \mathrm{~K}, 500 \mathrm{MHz}$ ) of the daisy chain dendrimer DC-G3 with the addition of TBAA (10 equiv. for each [c2]daisy chain unit).

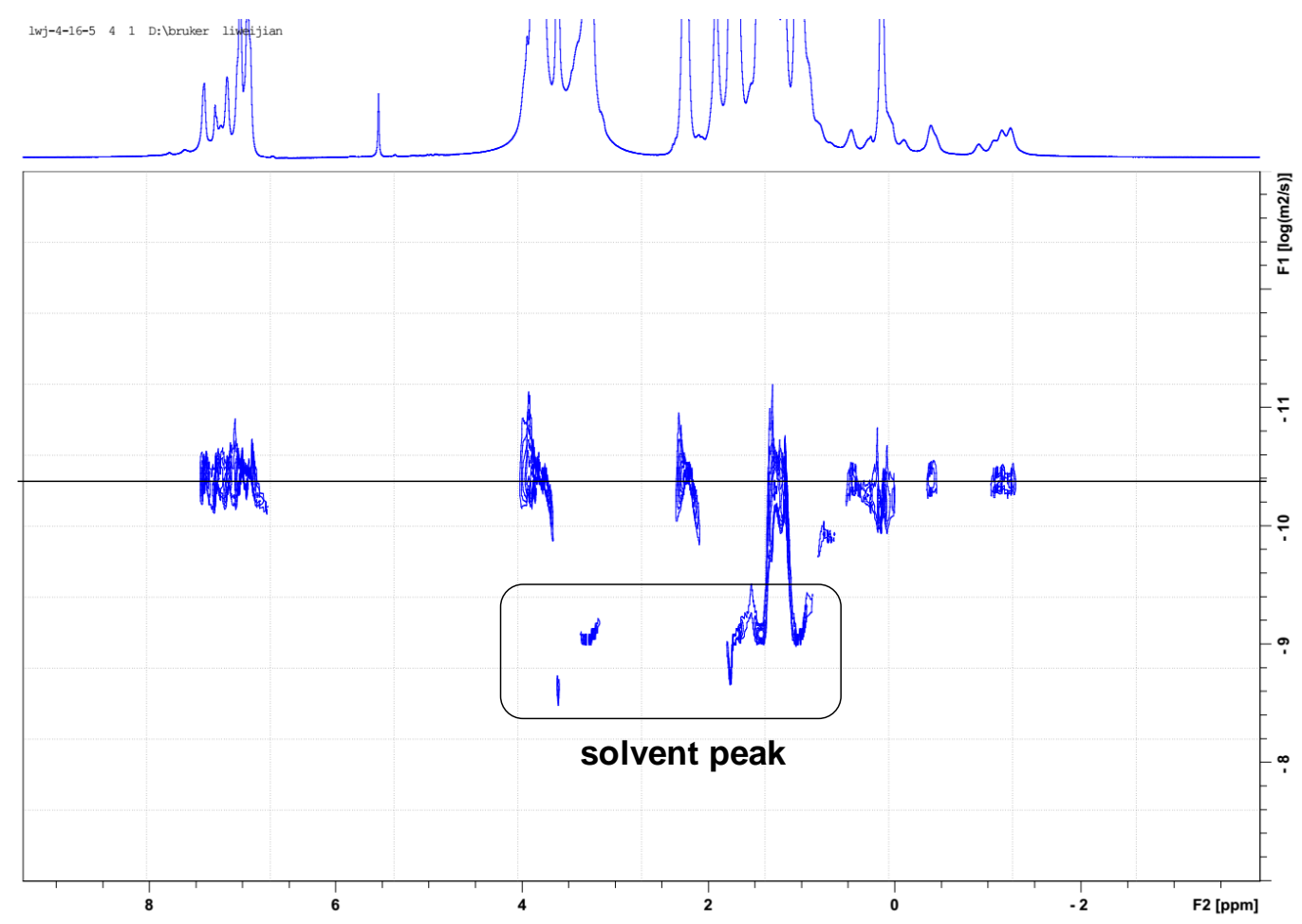

Figure S89: 2-D DOSY spectrum (THF- $\left.d_{8}, 298 \mathrm{~K}, 500 \mathrm{MHz}\right)$ of the mixture after adding $\mathrm{NaPF}_{6}$ into the solution of the daisy chain dendrimer DC-G3 and TBAA. 


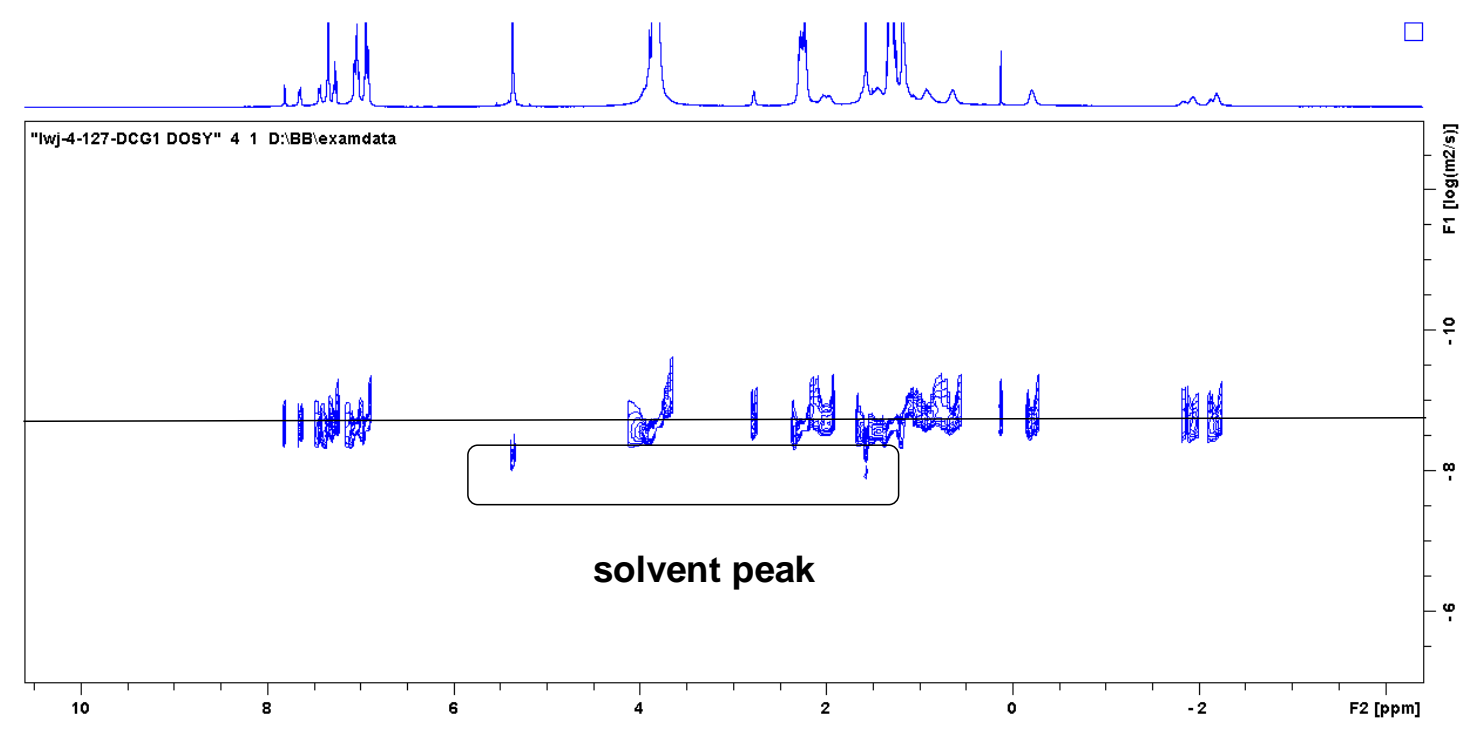

Figure S90: 2-D DOSY spectrum $\left(\mathrm{CD}_{2} \mathrm{Cl}_{2}, 298 \mathrm{~K}, 500 \mathrm{MHz}\right)$ of the daisy chain dendrimer DC-G1.

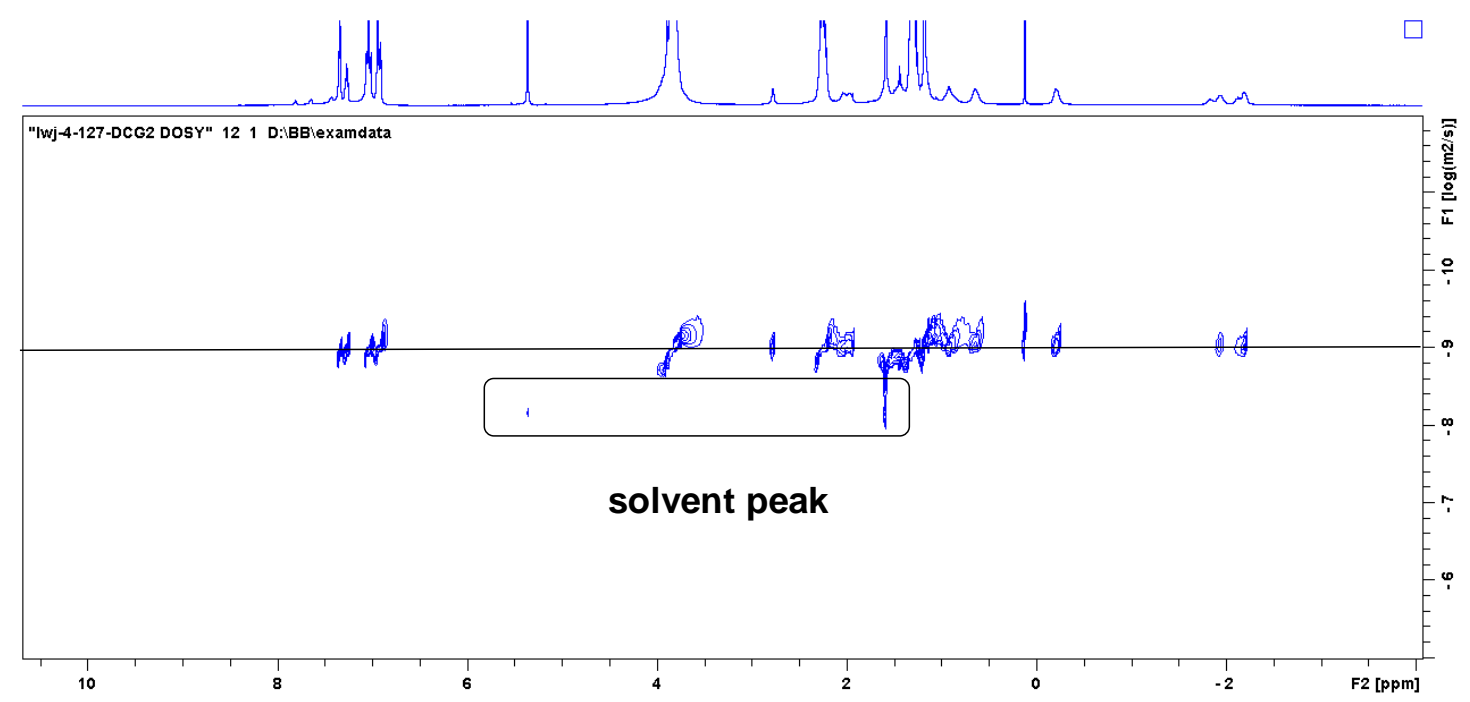

Figure S91: 2-D DOSY spectrum $\left(\mathrm{CD}_{2} \mathrm{Cl}_{2}, 298 \mathrm{~K}, 500 \mathrm{MHz}\right)$ of the daisy chain dendrimer DC-G2. 


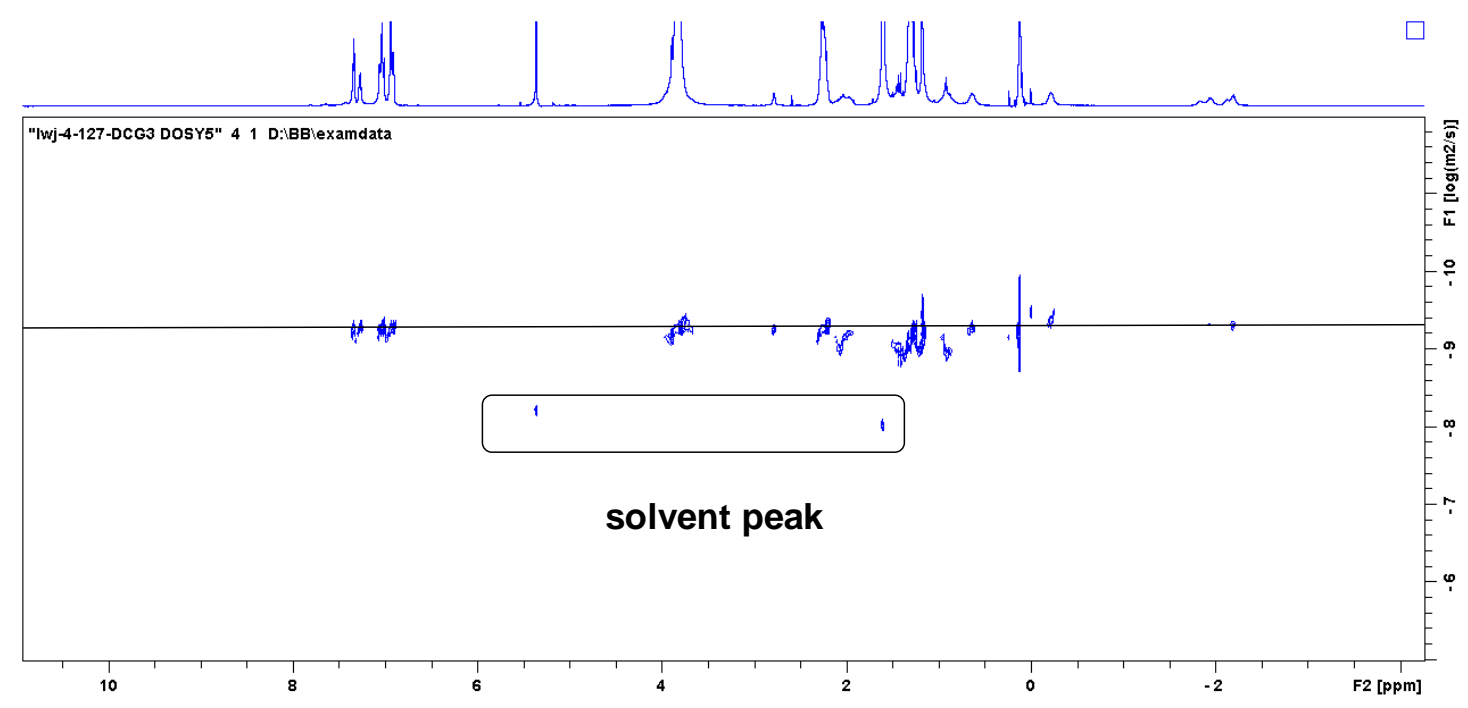

Figure S92: 2-D DOSY spectrum $\left(\mathrm{CD}_{2} \mathrm{Cl}_{2}, 298 \mathrm{~K}, 500 \mathrm{MHz}\right)$ of the daisy chain dendrimer DC-G3.

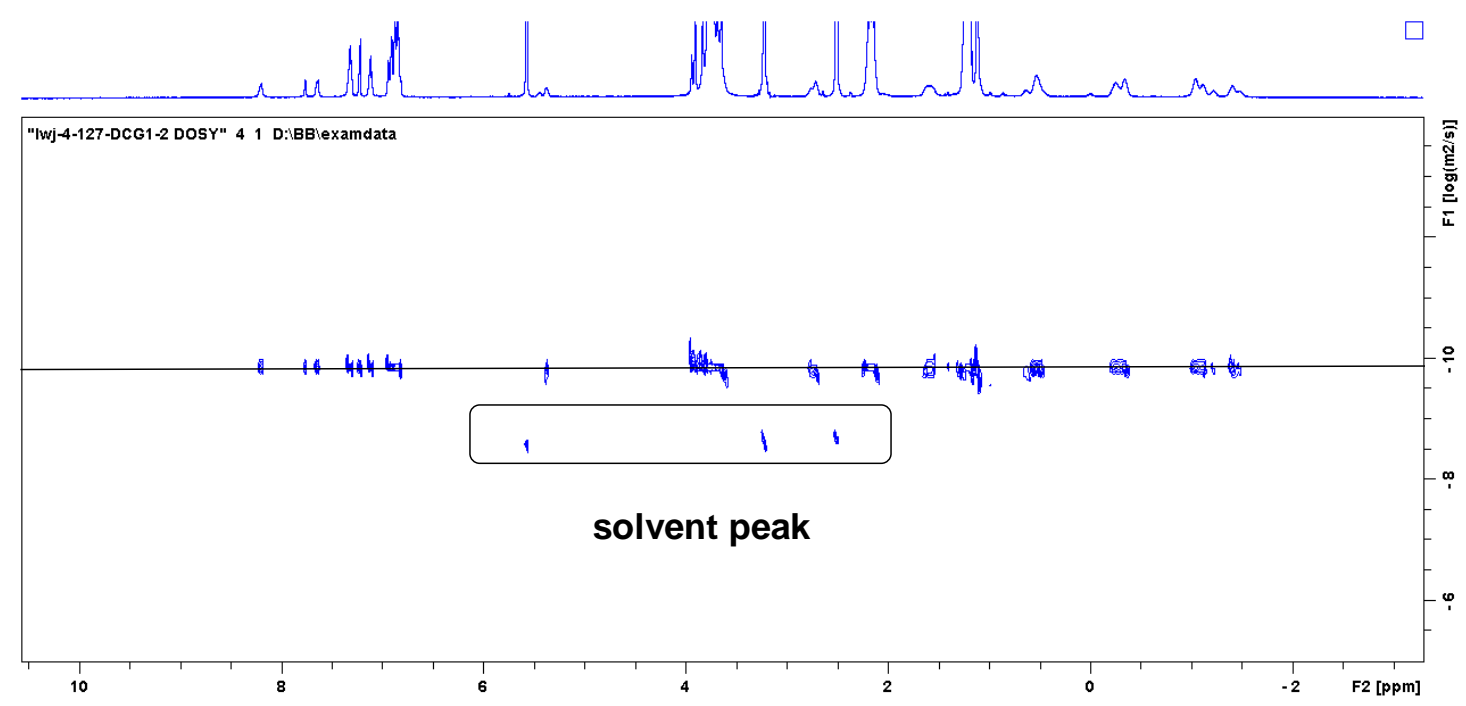

Figure S93: 2-D DOSY spectrum $\left(\mathrm{CD}_{2} \mathrm{Cl}_{2}, 298 \mathrm{~K}, 500 \mathrm{MHz}\right)$ of the daisy chain dendrimer DC-G1 with the addition of DMSO- $d_{6}(\mathrm{DMSO} / \mathrm{DCM}=2 / 1(\mathrm{v} / \mathrm{v}))$. 


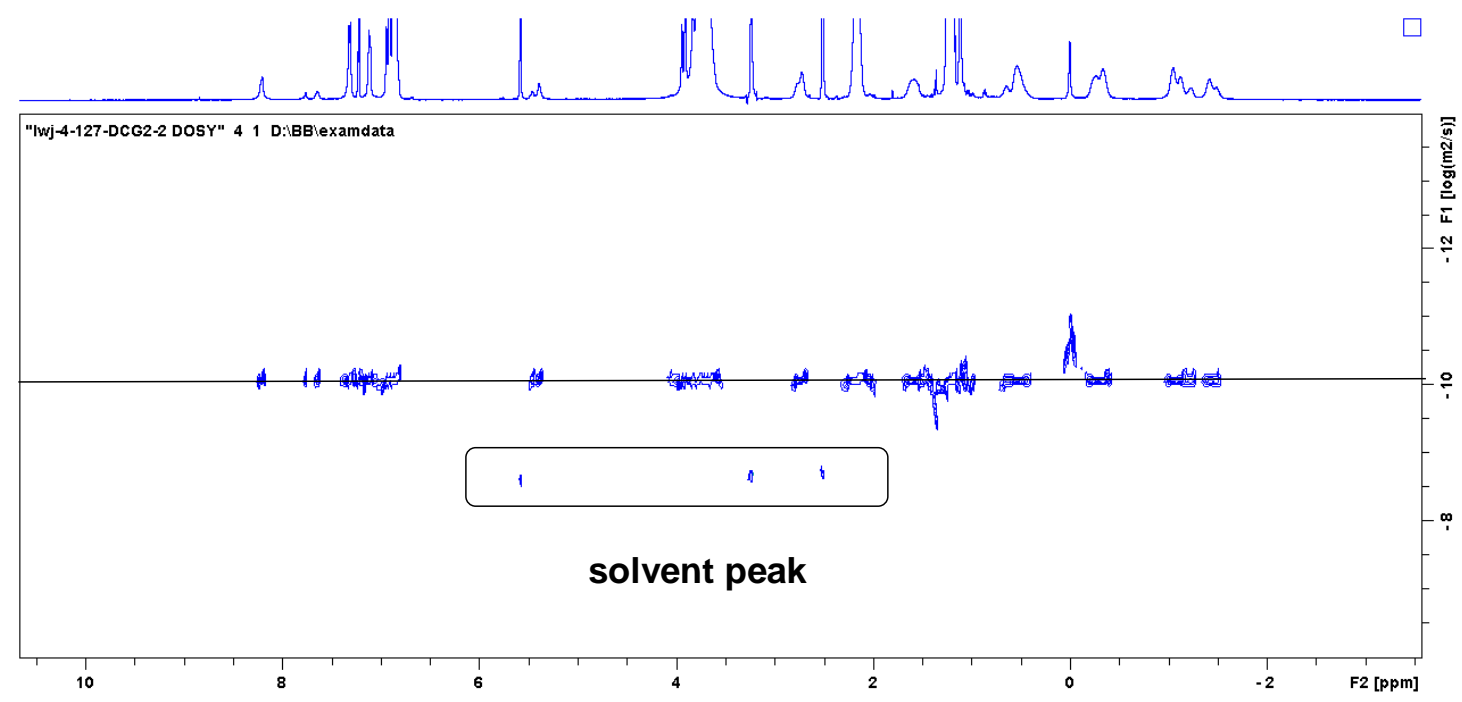

Figure S94: 2-D DOSY spectrum $\left(\mathrm{CD}_{2} \mathrm{Cl}_{2}, 298 \mathrm{~K}, 500 \mathrm{MHz}\right)$ of the daisy chain dendrimer DC-G2 with the addition of DMSO- $d_{6}(\mathrm{DMSO} / \mathrm{DCM}=2 / 1(\mathrm{v} / \mathrm{v}))$.

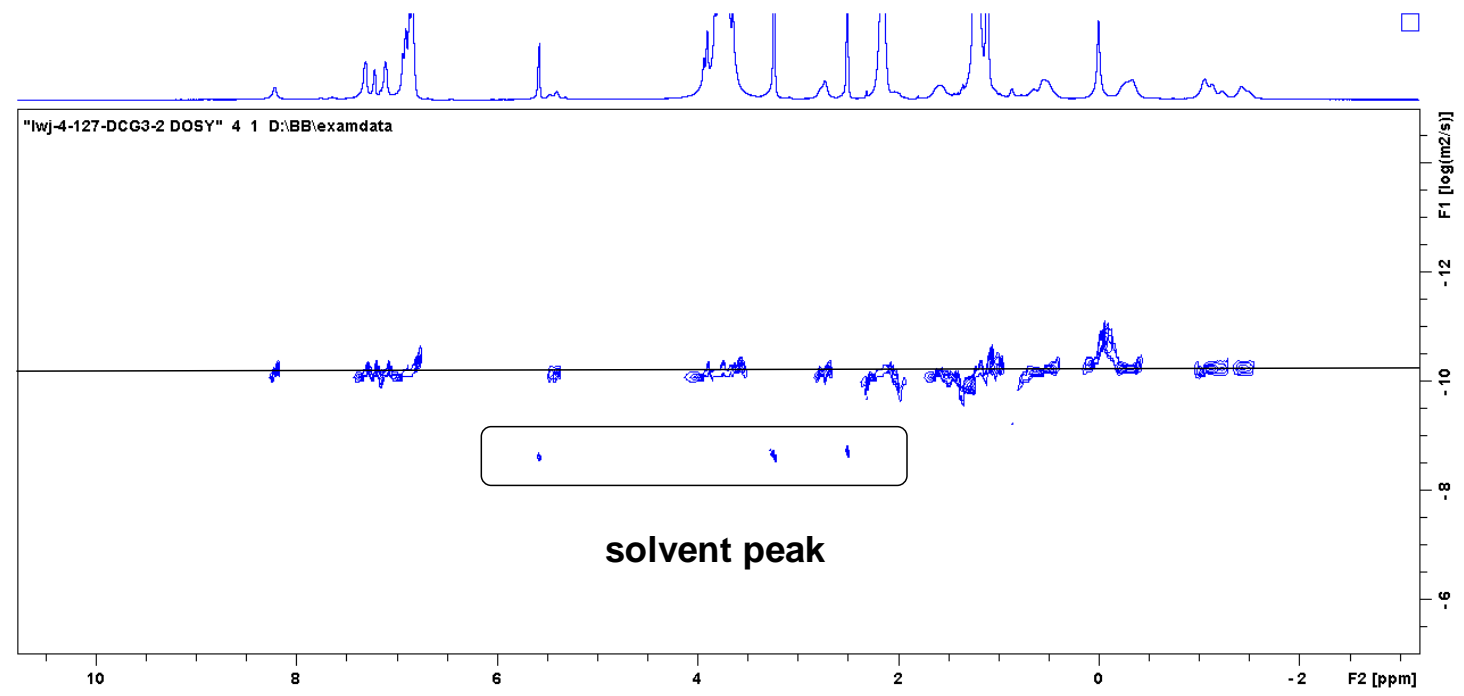

Figure S95: 2-D DOSY spectrum $\left(\mathrm{CD}_{2} \mathrm{Cl}_{2}, 298 \mathrm{~K}, 500 \mathrm{MHz}\right)$ of the daisy chain dendrimer DC-G3 with the addition of DMSO- $d_{6}(\mathrm{DMSO} / \mathrm{DCM}=2 / 1(\mathrm{v} / \mathrm{v}))$. 


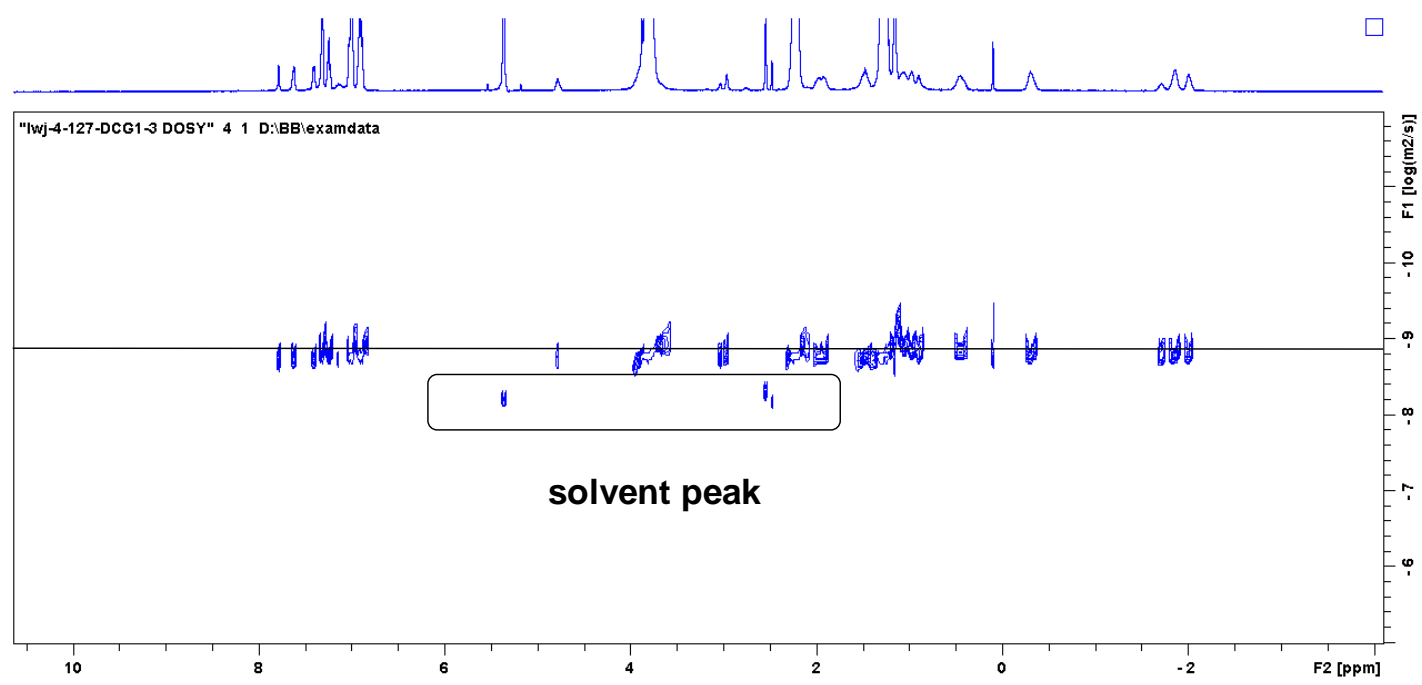

Figure S96: 2-D DOSY spectrum $\left(\mathrm{CD}_{2} \mathrm{Cl}_{2}, 298 \mathrm{~K}, 500 \mathrm{MHz}\right)$ of the mixture after adding $\mathrm{D}_{2} \mathrm{O}$ into the solution of the daisy chain dendrimer DC-G1 and DMSO- $d_{6}$.

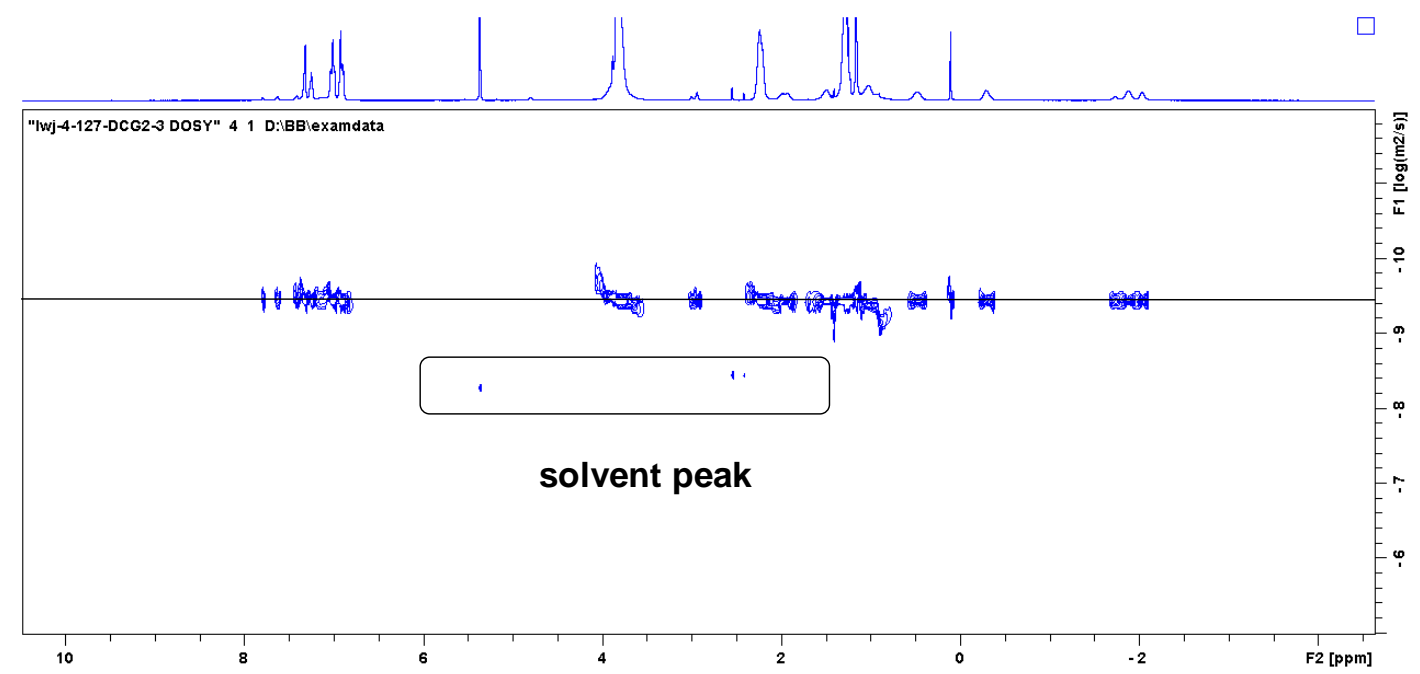

Figure S97: 2-D DOSY spectrum $\left(\mathrm{CD}_{2} \mathrm{Cl}_{2}, 298 \mathrm{~K}, 500 \mathrm{MHz}\right)$ of the mixture after adding $\mathrm{D}_{2} \mathrm{O}$ into the solution of the daisy chain dendrimer DC-G2 and DMSO- $d_{6}$.

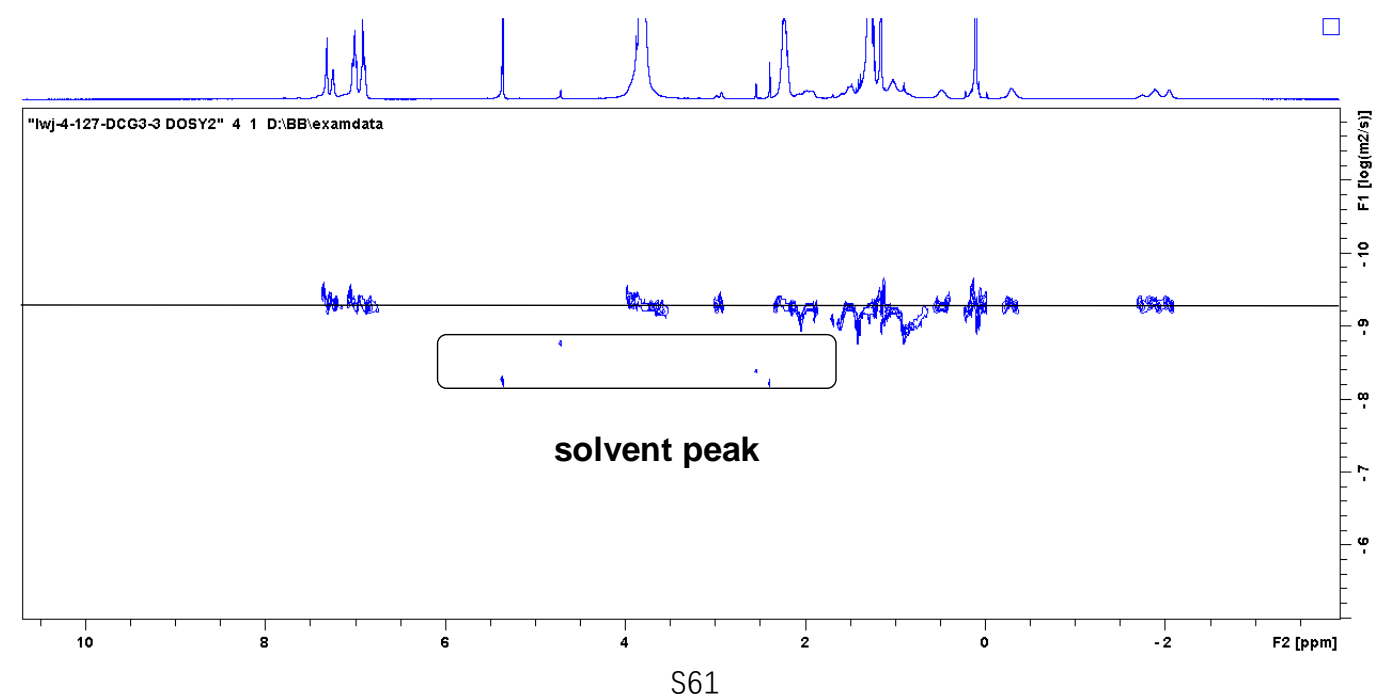


Figure S98: 2-D DOSY spectrum $\left(\mathrm{CD}_{2} \mathrm{Cl}_{2}, 298 \mathrm{~K}, 500 \mathrm{MHz}\right)$ of the mixture after adding $\mathrm{D}_{2} \mathrm{O}$ into the solution of the daisy chain dendrimer DC-G3 and DMSO- $d_{6}$.

Table S2: DLS data of anion-induced dimension modulation of the daisy chain dendrimers DC-G1, DC-G2 and DC-G3.

\begin{tabular}{|c|c|c|c|}
\hline & DC-G1 & DC-G1 + TBAA & $\mathrm{DC}-\mathrm{G} 1+\mathrm{TBAA}+\mathrm{Na}^{+}$ \\
\hline \multirow[t]{2}{*}{ Size (d.nm) } & $3.8 \pm 0.1$ & $2.8 \pm 0.1$ & $3.7 \pm 0.2$ \\
\hline & DC-G2 & DC-G2 + TBAA & $\mathrm{DC}-\mathrm{G} 2+\mathrm{TBAA}+\mathrm{Na}^{+}$ \\
\hline \multirow[t]{2}{*}{ Size (d.nm) } & $7.4 \pm 0.1$ & $5.1 \pm 0.2$ & $7.0 \pm 0.1$ \\
\hline & DC-G3 & DC-G3 + TBAA & $\mathrm{DC}-\mathrm{G} 3+\mathrm{TBAA}+\mathrm{Na}^{+}$ \\
\hline Size (d.nm) & $13.5 \pm 0.2$ & $7.2 \pm 0.1$ & $13.0 \pm 0.2$ \\
\hline
\end{tabular}

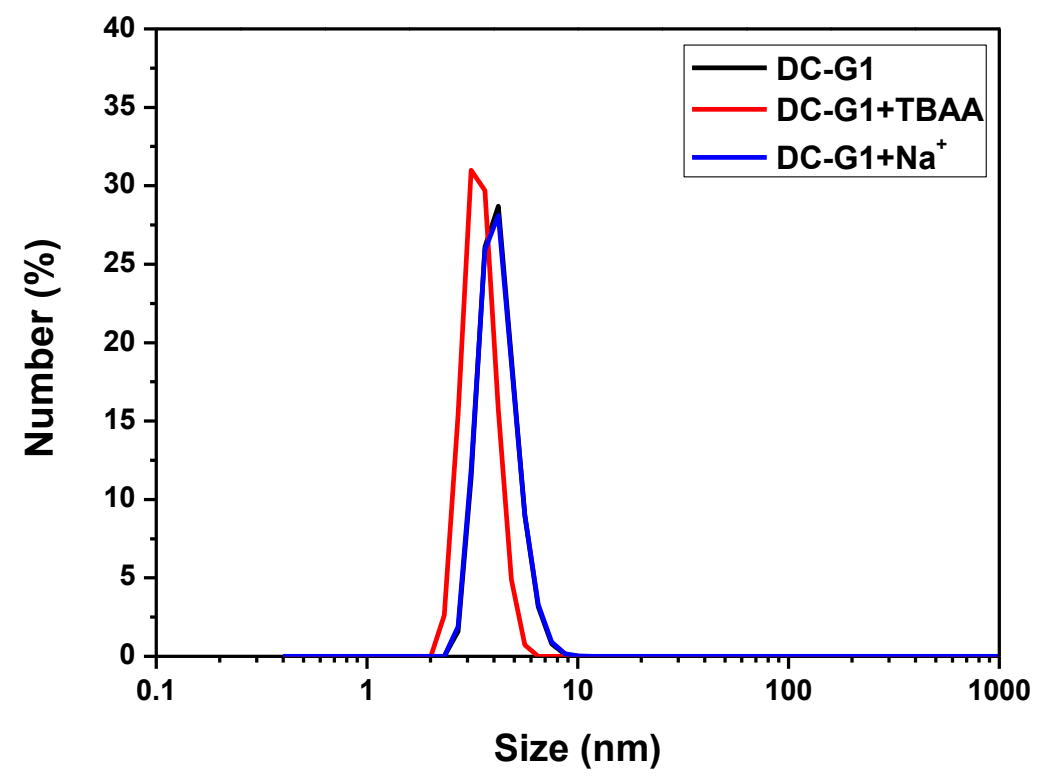

Figure S99: DLS spectra of anion-induced dimension modulation of daisy chain dendrimer DC-G1. 


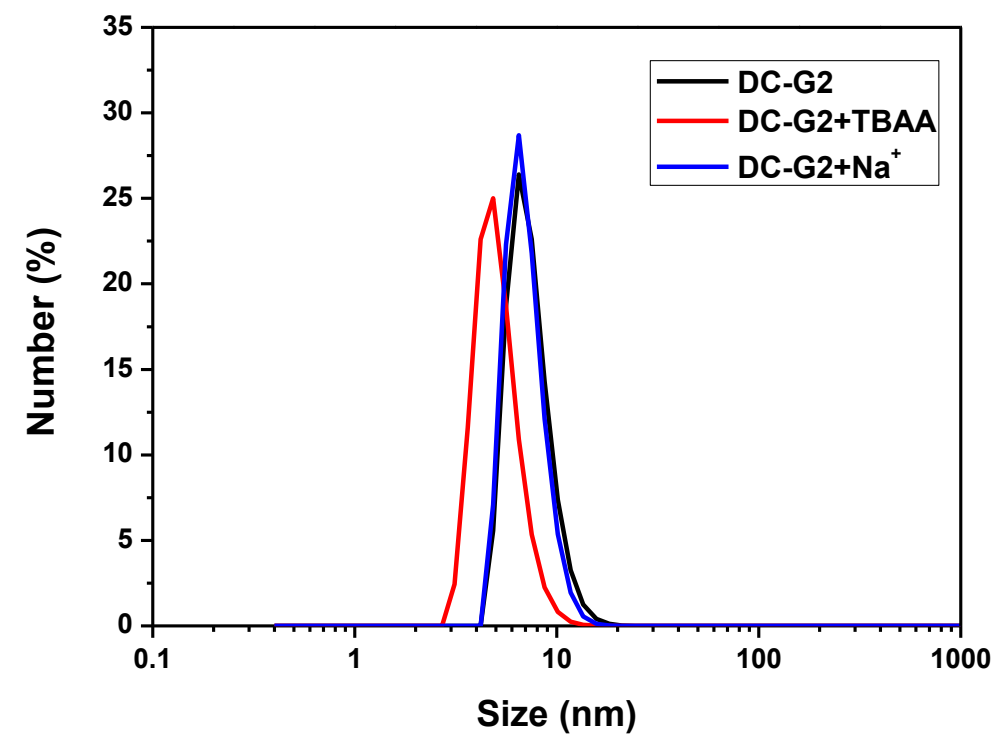

Figure S100: DLS spectra of anion-induced dimension modulation of daisy chain dendrimer DC-G2.

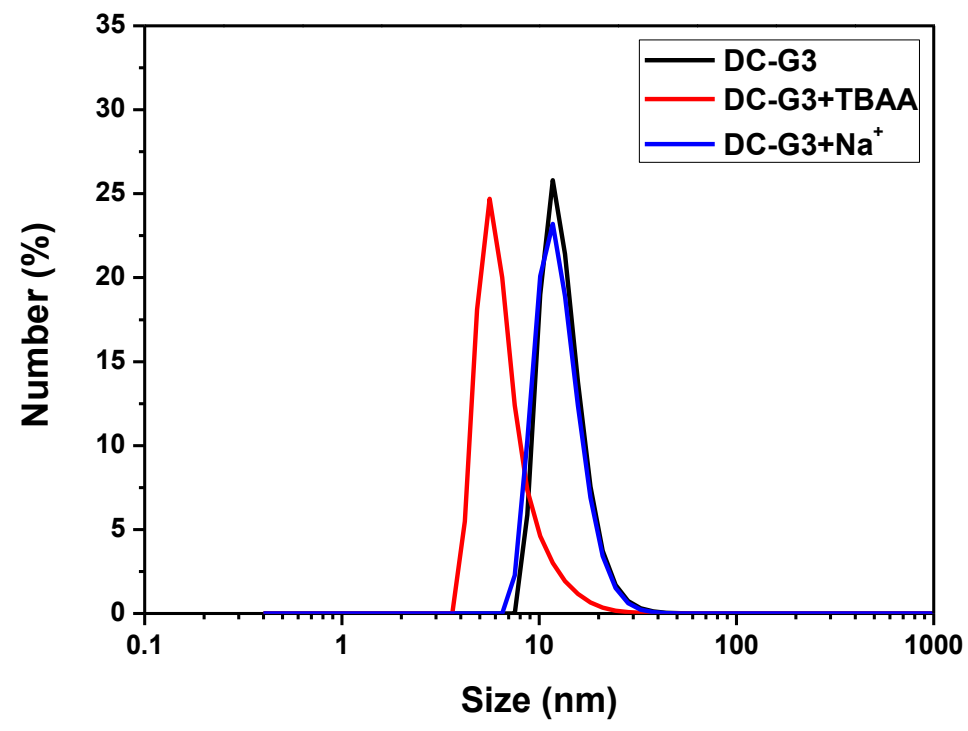

Figure S101: DLS spectra of anion-induced dimension modulation of daisy chain dendrimer DC-G3. 
Table S3: DLS data of solvent-induced dimension modulation of the daisy chain dendrimers DC-G1, DC-G2 and DC-G3.

\begin{tabular}{|c|c|c|c|}
\hline & DCM & DMSO/DCM 2/1 & Removing DMSO \\
\hline $\begin{array}{c}\text { DC-G1 } \\
\text { Size (d.nm) }\end{array}$ & $3.3 \pm 0.1$ & $2.2 \pm 0.1$ & $3.1 \pm 0.2$ \\
\hline $\begin{array}{c}\text { DC-G2 } \\
\text { Size (d.nm) }\end{array}$ & $7.5 \pm 0.2$ & $4.8 \pm 0.1$ & $6.9 \pm 0.2$ \\
\hline $\begin{array}{c}\text { DC-G3 } \\
\text { Size (d.nm) }\end{array}$ & $13.1 \pm 0.1$ & $6.9 \pm 0.1$ & $12.6 \pm 0.3$ \\
\hline
\end{tabular}

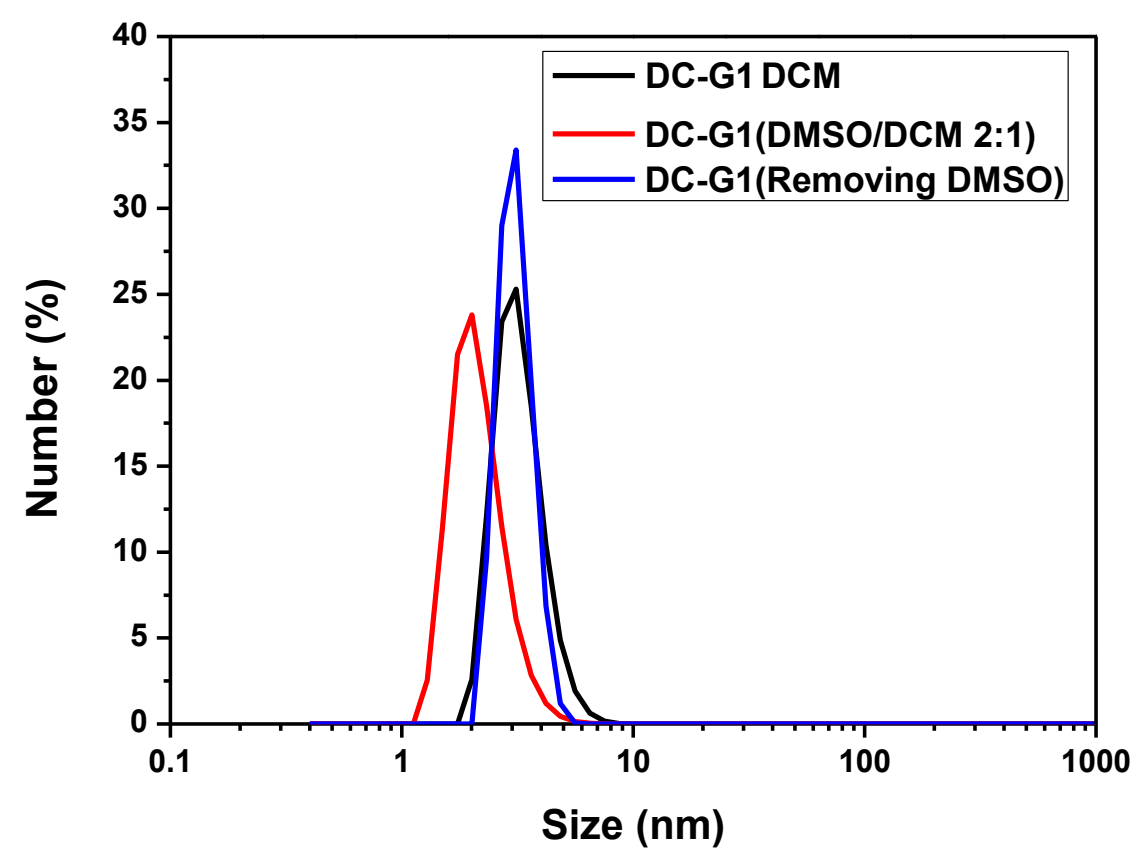

Figure S102: DLS spectra of solvent-induced dimension modulation of daisy chain dendrimer DC-G1. 


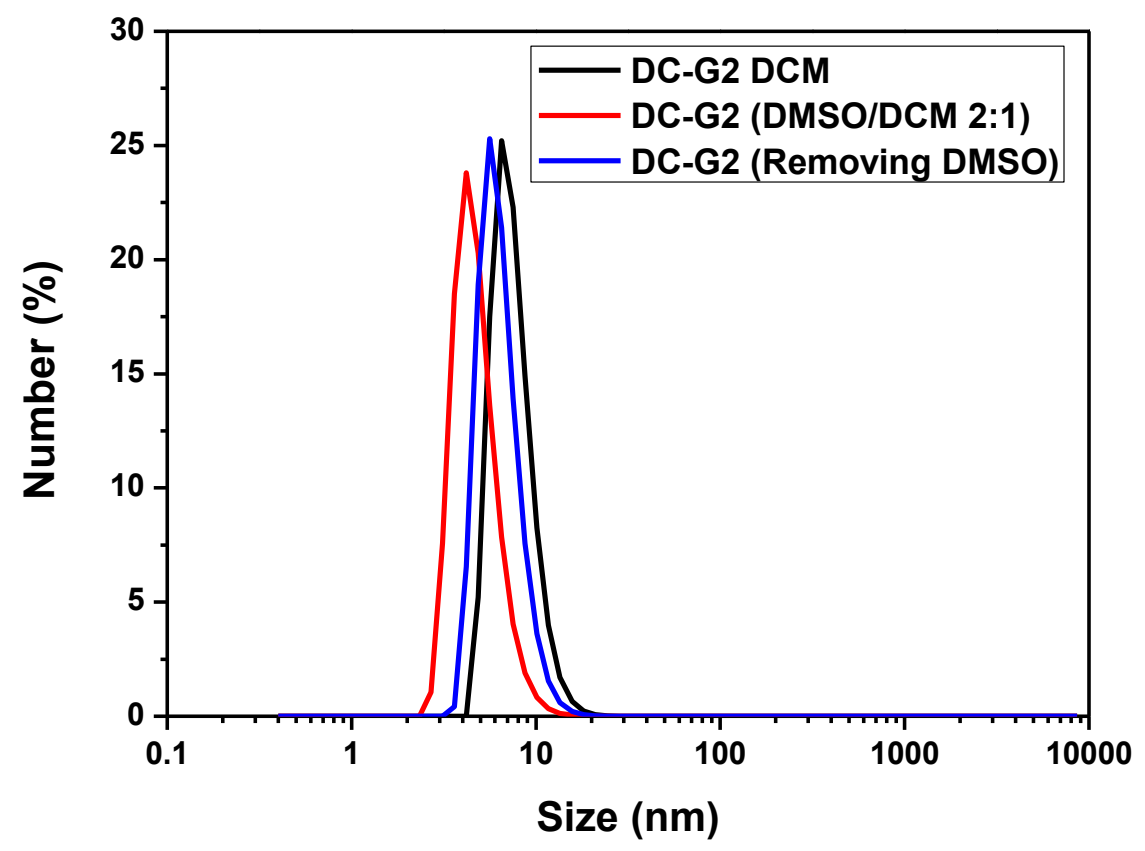

Figure S103: DLS spectra of solvent-induced dimension modulation of daisy chain dendrimer DC-G2.

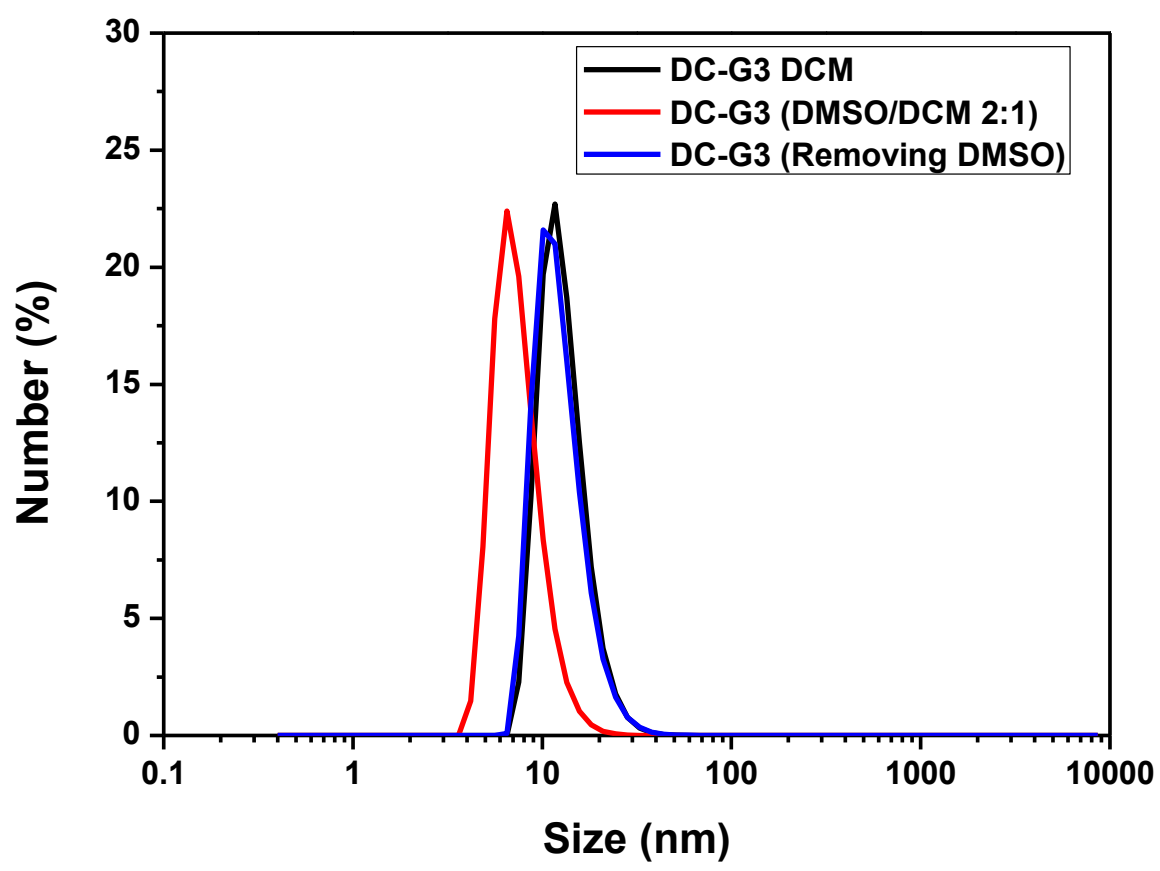

Figure S104: DLS spectra of solvent-induced dimension modulation of daisy chain dendrimer DC-G3. 
Section 6. Synthesis and characterization of the model dendrimer
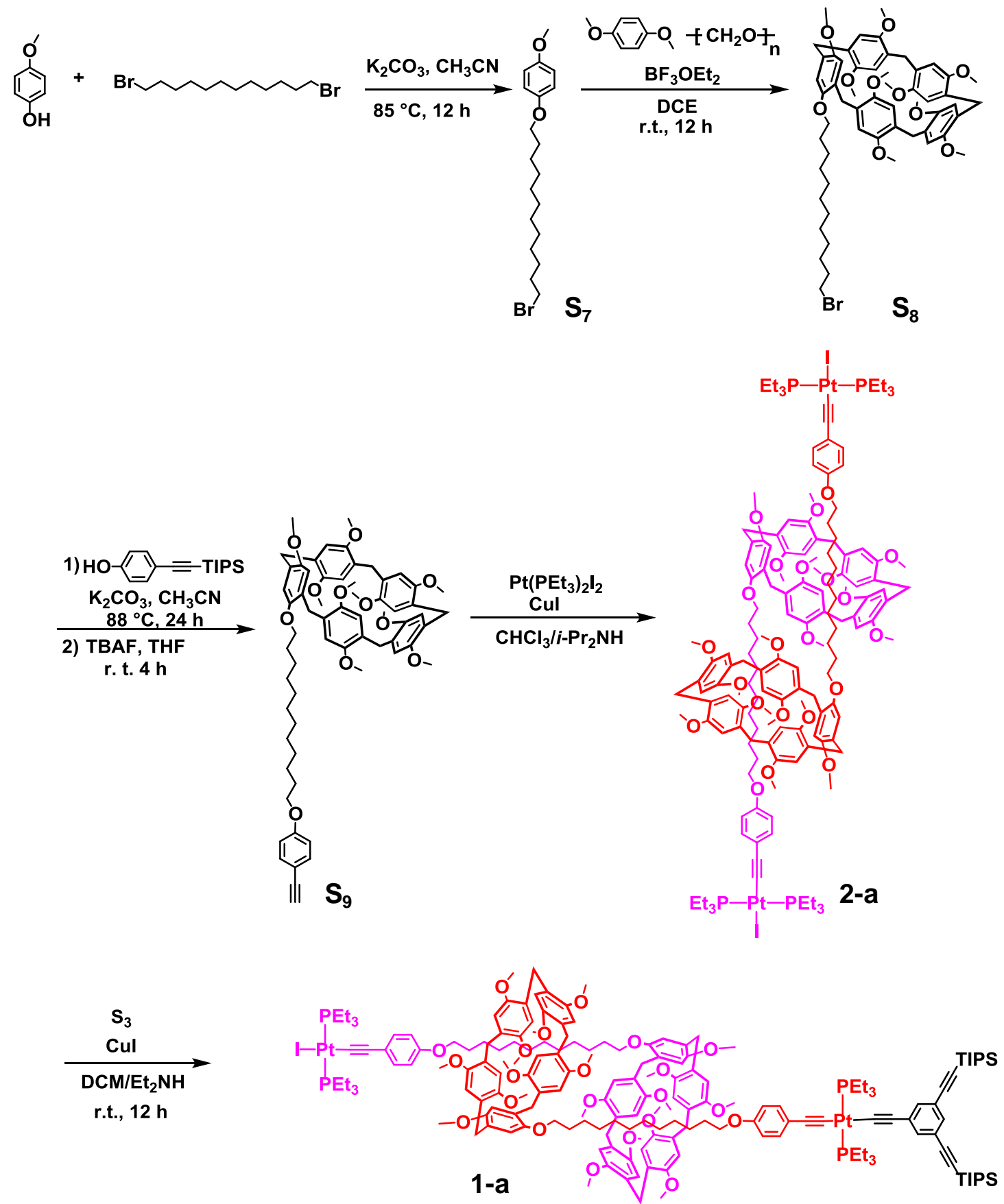

Scheme S5: The synthesis route of the model [c2]daisy chain rotaxane 1-a without urea moiety as the stimuli-responsive site. 


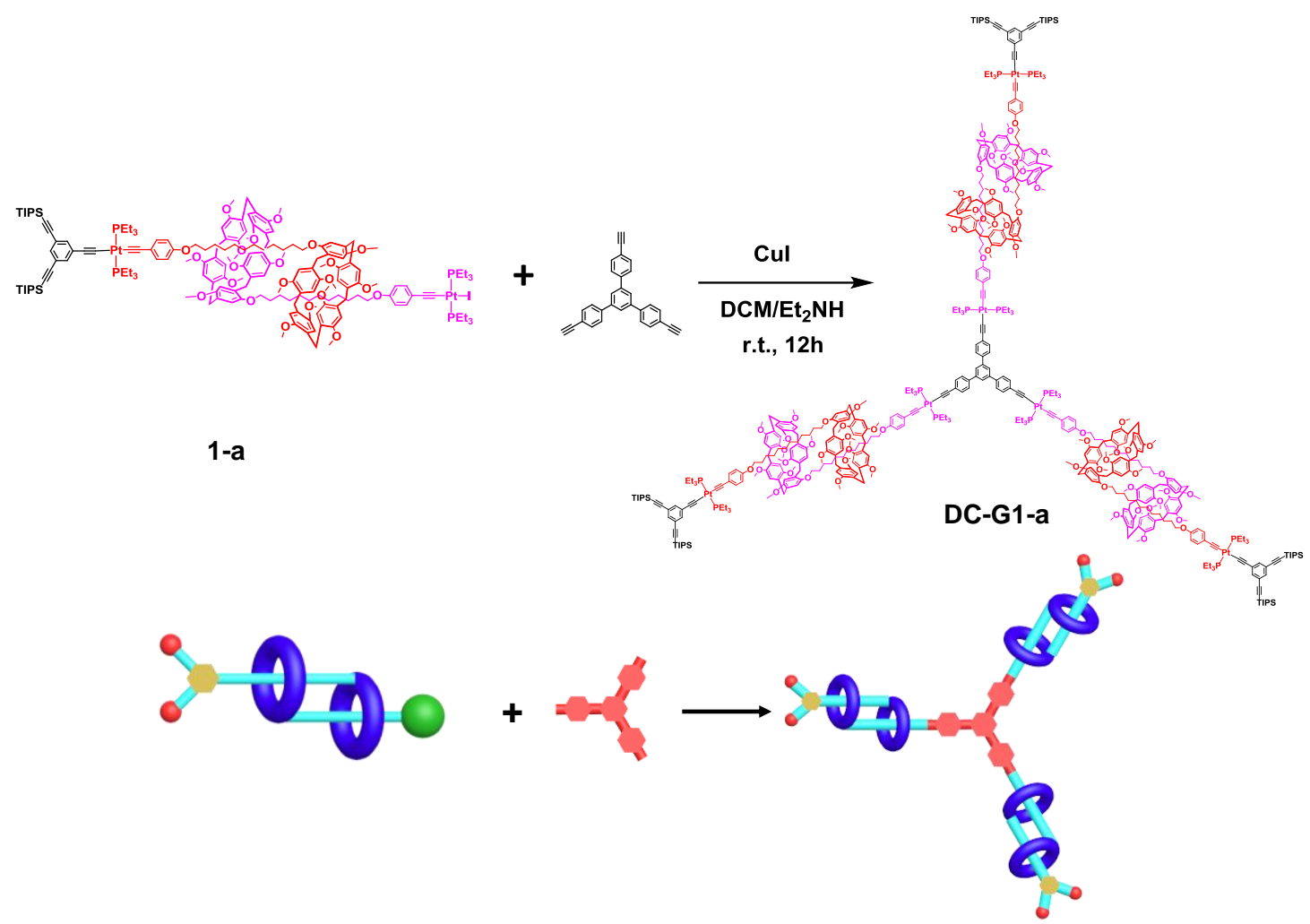

Scheme S6: The synthesis route of the model first-generation model daisy chain dendrimer DC-G1-a from corresponding building block 1-a.

Synthesis of S9: To a stirred mixture of compound $\mathbf{S}_{8}(5.00 \mathrm{~g}, 5.08 \mathrm{mmol})$ and 4-[(triisopropylsilyl)ethynyl]phenol $(2.79 \mathrm{~g}, 10.16 \mathrm{mmol})$ in acetonitrile (pre-dried by $\left.\mathrm{Na}_{2} \mathrm{SO}_{4}, 250 \mathrm{~mL}\right), \mathrm{K}_{2} \mathrm{CO}_{3}(2.81 \mathrm{~g}, 20.32 \mathrm{mmol}$ ) was added. The resultant suspension was refluxed for $24 \mathrm{~h}$. After cooling to room temperature, the reaction mixture was filtered and the filtrate was concentrated in vacuo to give a yellow solid residue. The resulting crude mixture was used directly as the starting materials during the next step. A solution of the crude mixture from the previous step in THF $(100 \mathrm{~mL})$ and then a solution of $\mathrm{Bu}_{4} \mathrm{NF}(1.0 \mathrm{M}, 3.5 \mathrm{~mL})$ in THF was added dropwise into the reaction flask. The reaction mixture was stirred at room temperature for $4 \mathrm{~h}$. The obtained residue was washed by water, then dried with $\mathrm{Na}_{2} \mathrm{SO}_{4}$ and concentrated. The residue was further purified by column chromatography ( $\mathrm{SiO} 2 ; \mathrm{PE} / \mathrm{DCM})$ to afford a white solid (3.32 g, 64\% over 2 steps). ${ }^{1} \mathrm{H}$ NMR $\left(500 \mathrm{MHz}, \mathrm{CD}_{2} \mathrm{Cl}_{2}\right) \delta 7.49-7.52(\mathrm{~m}, 2 \mathrm{H})$, 6.97-7.00 (m, 10H), 6.92-6.95 (m, 2H), 4.02-4.05 (t, $J=7.5 \mathrm{~Hz}, 2 \mathrm{H}), 3.96-3.99(\mathrm{t}, J=$ $7.5 \mathrm{~Hz}, 2 \mathrm{H}), 3.82-3.86(\mathrm{~m}, 37 \mathrm{H}), 3.14(\mathrm{~s}, 1 \mathrm{H}), 1.91-1.97(\mathrm{~m}, 2 \mathrm{H}), 1.82-1.86(\mathrm{~m}, 2 \mathrm{H})$, $1.62-1.68(\mathrm{~m}, 2 \mathrm{H}), 1.42-1.52(\mathrm{~m}, 2 \mathrm{H}) .{ }^{13} \mathrm{C} \mathrm{NMR}\left(126 \mathrm{MHz}, \mathrm{CD}_{2} \mathrm{Cl}_{2}\right) \delta 161.19,151.82$, 151.79 , 151.78, 151.70, 151.20, 135.01, 129.80, 129.76, 129.68, 129.66, 129.61, $129.60,129.52$, 115.94, 115.54, 115.20, 114.72, 114.69, 114.66, 114.55, 85.09, 77.10, 
69.79, 69.63, 56.94, 56.92, 56.91, 56.88, 56.80, 31.43, 31.20, 31.19, 31.17, 31.15, $31.14, \quad 30.94, \quad 30.73, \quad 30.69, \quad 30.64, \quad 30.51, \quad 30.41, \quad 27.91, \quad 27.51$. HRMS (MALDI-TOF-MS): Calculated for [S9] $]^{+}: m / z=1020.5388$; Found: $m / z=1020.5407$. Calculated for $[\mathbf{S 9}+\mathrm{Na}]^{+}: m / z=1043.5285$; Found: $m / z=1043.5326$.

Synthesis of 2-a: A Schlenk flask was charged with $400 \mathrm{mg}(0.39 \mathrm{mmol})$ of $\mathbf{S} 9$ and $\mathrm{Pt}\left(\mathrm{PEt}_{3}\right)_{2} \mathrm{I}_{2}(1.34 \mathrm{~g}, 1.96 \mathrm{mmol})$. The Schlenk flask was then evacuated and back-filled with $\mathrm{N}_{2}$ three times. Next, $6 \mathrm{~mL}$ of the mixture solvent of anhydrous and degassed $\mathrm{CHCl}_{3}$ and $i-\mathrm{Pr}_{2} \mathrm{NH}(\mathrm{v} / \mathrm{v}, 2: 1)$ was added via syringe. After stirring at $-35{ }^{\circ} \mathrm{C}$ for 17 hours, $\mathrm{CuI}(15 \mathrm{mg})$ was added to the mixture under an inert atmosphere and the mixture was stirred for 48 hours under $-35{ }^{\circ} \mathrm{C}$ continuously. Then the mixture was allowed to warm to room temperature and stirred for 48 hours. The solution was concentrated and the residue was purified by column chromatography $\left(\mathrm{SiO}_{2}\right.$; DCM/EA) and gel permeation chromatography (GPC). A pale yellow solid 2-a (408 $\mathrm{mg}, 66 \%$ yield) was obtained. ${ }^{1} \mathrm{H}$ NMR $\left(500 \mathrm{MHz}, \mathrm{CD}_{2} \mathrm{Cl}_{2}\right) \delta$ 7.27-7.28 (m, 4H), 6.91-6.99 (m, 20H), 6.78-6.79 (m, 4H), 3.74-3.96 (m, 78H), 2.82-2.90 (m, 4H), 2.28-2.31 (m, 24H), $1.94(\mathrm{~m}, 4 \mathrm{H}), 1.49(\mathrm{~m}, 4 \mathrm{H}), 1.20-1.31(\mathrm{~m}, 40 \mathrm{H}), 1.01(\mathrm{~m}, 4 \mathrm{H})$, $0.72(\mathrm{~m}, 4 \mathrm{H}), 0.33(\mathrm{~m}, 4 \mathrm{H}),-0.30-(-0.26)(\mathrm{m}, 4 \mathrm{H}),-1.25(\mathrm{~m}, 4 \mathrm{H}),-1.65(\mathrm{~m}, 4 \mathrm{H}) .{ }^{31} \mathrm{P}$ NMR (202 MHz, $\left.\mathrm{CD}_{2} \mathrm{Cl}_{2}\right) \delta$ 8.94. ${ }^{13} \mathrm{C} \mathrm{NMR}\left(126 \mathrm{MHz}, \mathrm{CD}_{2} \mathrm{Cl}_{2}\right) \delta$ 159.21, 159.11, $152.02,151.94,151.88,151.85,151.79,151.44,133.28,133.11,129.75,129.72$, $129.67,129.64,129.59,129.53,129.49,129.45,121.71,115.68,115.58,115.47$, $115.07,114.98,114.85,114.77,114.71,114.55,114.48,114.43,114.36,114.30$, 101.16, 88.00, 70.26, 70.20, 69.99, 57.11, 57.04, 57.03, 56.88, 56.84, 56.78, 56.76, $56.67,56.66,56.62,56.56,32.42,32.35,32.27,32.24,32.17,32.14,31.62,30.57$, $30.49,30.34,30.09$, 30.05, 28.36, 28.30, 24.63, 18.32, 18.18, 18.04, 16.24, 16.11, 15.97, 9.65, 9.39. LRMS (MALDI-TOF-MS): Calculated for $[2-\mathbf{a}+\mathrm{H}]^{+}: m / z=3156.2$; Found: $m / z=3156.5$.

Synthesis of 1-a: A Schlenk flask was charge with $\mathbf{S} 3$ (67 $\mathrm{mg}, 0.14 \mathrm{mmol})$ and compound 2-a (920 mg, $0.29 \mathrm{mmol})$. The Schlenk flask was then evacuated via reduced pressure and backfilled with $\mathrm{N}_{2}$ for three times. Next, dichloromethane (20 $\mathrm{mL})$ and diethylamine $(10 \mathrm{~mL})$ was added via syringe. The resultant solution was stirred for 2 hours at room temperature. Then $\mathrm{CuI}(3 \mathrm{mg})$ was added to the mixture under $\mathrm{N}_{2}$ atmosphere, and the mixture was allowed to stir for 12 hours. The solvent was then removed by reduced pressure, and the compound was purified by column chromatography $\left(\mathrm{SiO}_{2} ; \mathrm{DCM} / \mathrm{EA}\right)$ and gel permeation chromatography (GPC). A pale yellow solid 1-a (290 mg, 57\% yield) was obtained. ${ }^{1} \mathrm{H}$ NMR $\left(500 \mathrm{MHz}, \mathrm{CD}_{2} \mathrm{Cl}_{2}\right) \delta$ 7.31-7.32 (m, 3H), 7.22-7.26 (m, 4H), 6.88-6.96 (m, 20H), 6.73-6.76 (m, 4H), $3.72-3.93(\mathrm{~m}, 78 \mathrm{H}), 2.80-2.85(\mathrm{~m}, 4 \mathrm{H}), 2.19-2.28(\mathrm{~m}, 24 \mathrm{H}), 1.94(\mathrm{~m}, 4 \mathrm{H}), 1.48(\mathrm{~m}$, $4 \mathrm{H}), 1.19-1.27(\mathrm{~m}, 40 \mathrm{H}), 1.15(\mathrm{~s}, 42 \mathrm{H}), 0.97(\mathrm{~m}, 4 \mathrm{H}), 0.70(\mathrm{~m}, 4 \mathrm{H}), 0.31(\mathrm{~m}, 4 \mathrm{H})$, 
$-0.31(\mathrm{~m}, 4 \mathrm{H}),-1.28(\mathrm{~m}, 4 \mathrm{H}),-1.68(\mathrm{~m}, 4 \mathrm{H}) .{ }^{31} \mathrm{P} \mathrm{NMR}\left(202 \mathrm{MHz}, \mathrm{CD}_{2} \mathrm{Cl}_{2}\right) \delta 11.85$, 8.94. ${ }^{13} \mathrm{C}$ NMR $\left(126 \mathrm{MHz}, \mathrm{CD}_{2} \mathrm{Cl}_{2}\right) \delta 159.23,158.98,152.03,151.94,151.91,151.89$, $151.87,151.79,151.45,151.44,135.61,133.28,133.12,132.80,129.73,129.72$, $129.65,129.61,129.53,129.50,129.46,125.01,122.10,115.69,115.59,115.46$, $115.08,114.99,114.87,114.77,114.72,114.56,114.49,114.44,114.37,114.31$, $107.74,92.52,88.01,70.27,70.21,70.01,69.96,57.16,57.11,57.05$, 57.03, 56.89, $56.85,56.79,56.77,56.68,56.66,56.63,56.57,32.43,32.38,32.36,32.31,32.28$, $32.18,32.15,31.63,31.27,30.58,30.51,30.35,30.09$, 28.37, 28.31, 24.62, 19.99,

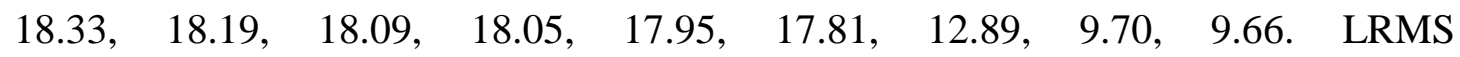
(MALDI-TOF-MS): Calculated for $[\mathbf{1 - a}+\mathrm{H}]^{+}: m / z=3493.2$; Found: $m / z=3493.2$.

Synthesis of the model first-generation daisy chain dendrimer DC-G1-a: A mixture of 1,3,5-tris(4-ethynylphenyl)benzene (3.28 mg, $0.0087 \mathrm{mmol}$ ) and daisy chain monomer 1-a (100 mg, $0.0286 \mathrm{mmol}$ ) were added in a Schlenk flask, the Schlenk flask was then evacuated and back-filled with $\mathrm{N}_{2}$ three times. Next, degassed dichloromethane/diethylamine (v/v, 1:1) $(10 \mathrm{~mL})$ and a catalytic amount of $\mathrm{CuI}$ were added under an inert atmosphere. The reaction was stirred for 12 hours at room temperature. The solvent was evaporated and the residue was purified by column chromatography $\left(\mathrm{SiO}_{2} ; \mathrm{DCM} / \mathrm{EA}\right)$ and gel permeation chromatography (GPC) to yield a pale-yellow solid DC-G1-a (45 mg, 49\%). ${ }^{1} \mathrm{H}$ NMR $\left(500 \mathrm{MHz}, \mathrm{CD}_{2} \mathrm{Cl}_{2}\right) \delta$ $7.78(\mathrm{~s}, 3 \mathrm{H}), 7.61-7.62(\mathrm{~m}, 6 \mathrm{H}), 7.40-7.41(\mathrm{~m}, 6 \mathrm{H}), 7.31-7.32(\mathrm{~m}, 9 \mathrm{H}), 7.24(\mathrm{~m}, 12 \mathrm{H})$, 6.88-6.96 (m, 60H), 6.73-6.76 (m, 12H), 3.71-3.93 (m, 234H), 2.78-2.83 (m, 12H), 2.18-2.26 (m, 72H), $1.92(\mathrm{~m}, 12 \mathrm{H}), 1.47(\mathrm{~m}, 12 \mathrm{H}), 1.23-1.31(\mathrm{~m}, 120 \mathrm{H}), 1.15(\mathrm{~s}, 126 \mathrm{H})$, $0.98(\mathrm{~m}, 12 \mathrm{H}), 0.71(\mathrm{~m}, 12 \mathrm{H}), 0.31(\mathrm{~m}, 12 \mathrm{H}),-0.32(\mathrm{~m}, 24 \mathrm{H}),-1.28(\mathrm{~m}, 12 \mathrm{H}),-1.68$ $(\mathrm{m}, 12 \mathrm{H}) .{ }^{31} \mathrm{P}$ NMR $\left(202 \mathrm{MHz}, \mathrm{CD}_{2} \mathrm{Cl}_{2}\right) \delta 11.84,11.77 .{ }^{13} \mathrm{C} \mathrm{NMR}\left(126 \mathrm{MHz}, \mathrm{CD}_{2} \mathrm{Cl}_{2}\right)$ $\delta$ 159.02, 152.04, 151.95, 151.91, 151.87, 151.79, 151.44, 143.61, 140.93, 135.60, $133.31,132.78,129.75,129.72,129.67,129.60,129.54,129.50,129.47,128.41$, $125.02,122.09,115.69,115.59,115.49,115.34,115.07,114.99,114.87,114.77$, $114.72,114.56,114.48,114.31,107.72,92.54,70.27,70.21,69.97,57.16,57.12$, $57.04,56.99,56.90,56.79,56.77,56.66,56.63,56.57,35.38,33.50,32.80,32.44$, $32.39,32.28,32.18,31.62,31.26,30.55,30.50,30.36,30.11,28.38,28.31,24.61$, 24.27, 19.98, 18.16, 18.10, 18.02, 17.96, 17.88, 17.82, 15.45, 12.89, 9.77, 9.71, 2.33. LRMS (MALDI-TOF-MS): Calculated for [DC-G1-a + Na $]^{+}: \mathrm{m} / z=10486.1$; Found: $m / z=10485.1$. 


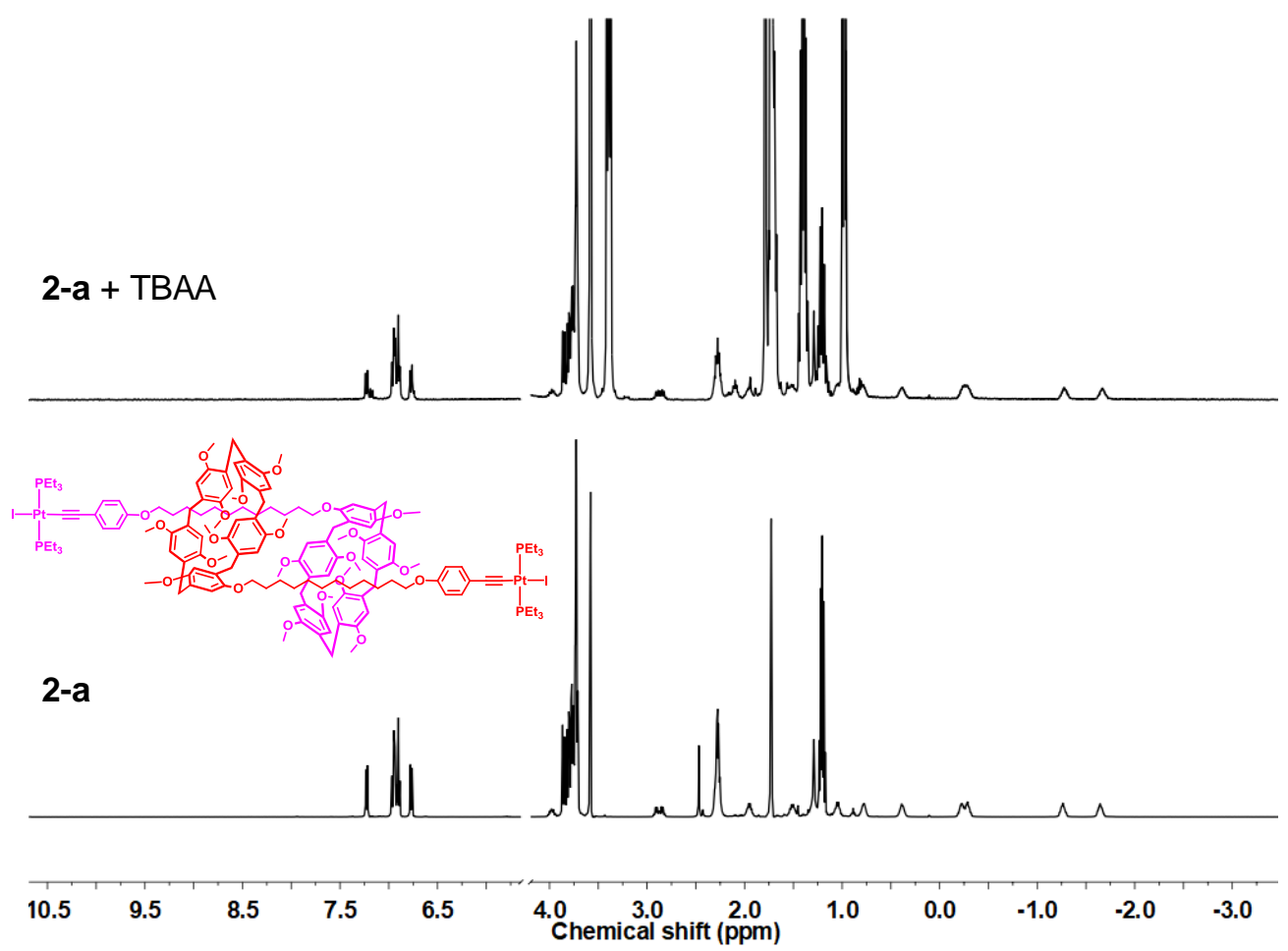

Figure S105: ${ }^{1} \mathrm{H}$ NMR spectra (THF- $d_{8}, 298 \mathrm{~K}, 500 \mathrm{MHz}$ ) of model complex 2-a (bottom); 2-a with the addition of TBAA (10 eq.) (top).

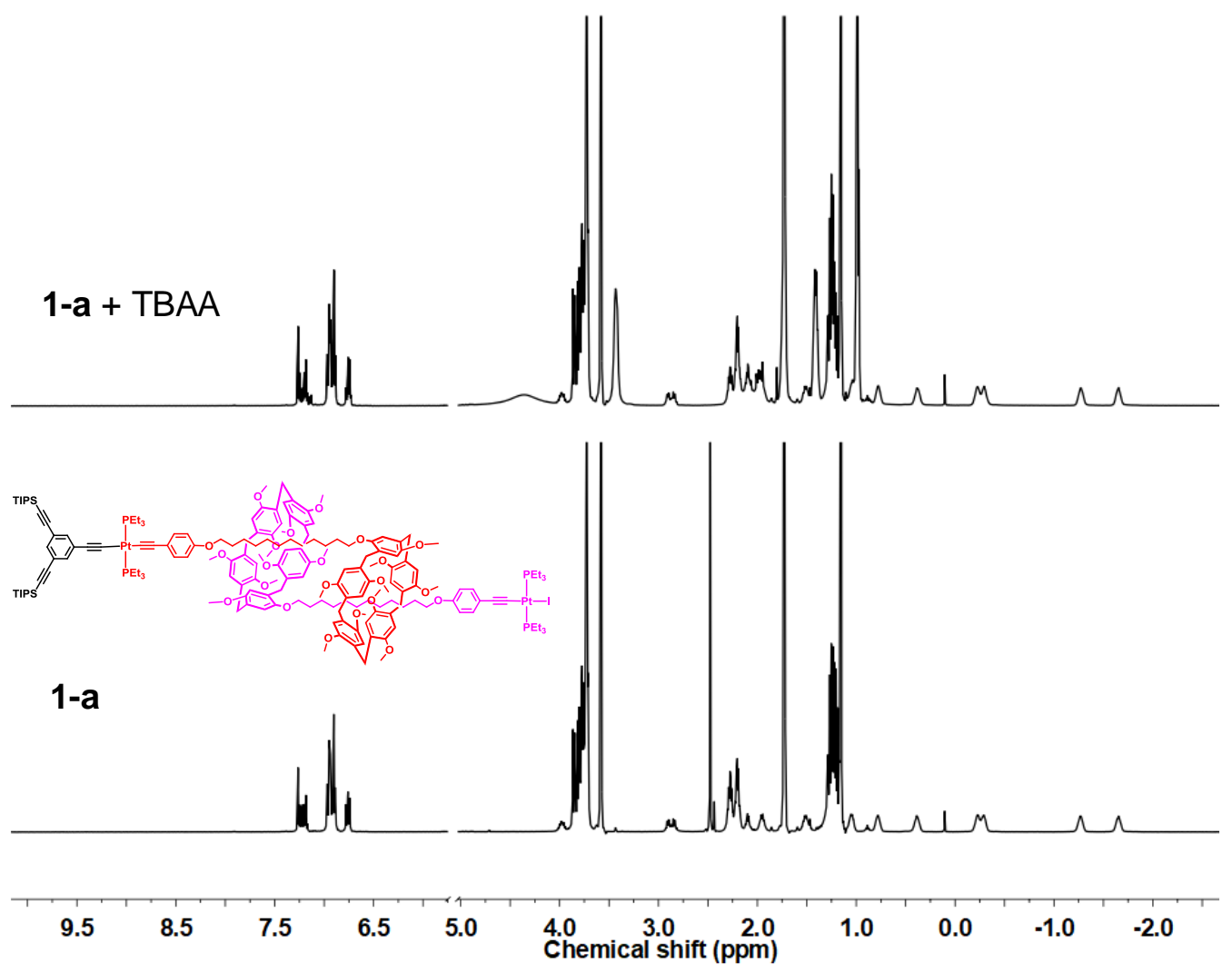

Figure S106: ${ }^{1} \mathrm{H}$ NMR spectra (THF- $d_{8}, 298 \mathrm{~K}, 500 \mathrm{MHz}$ ) of model complex 1-a (bottom); 1-a with the addition of TBAA (10 eq.) (top). 


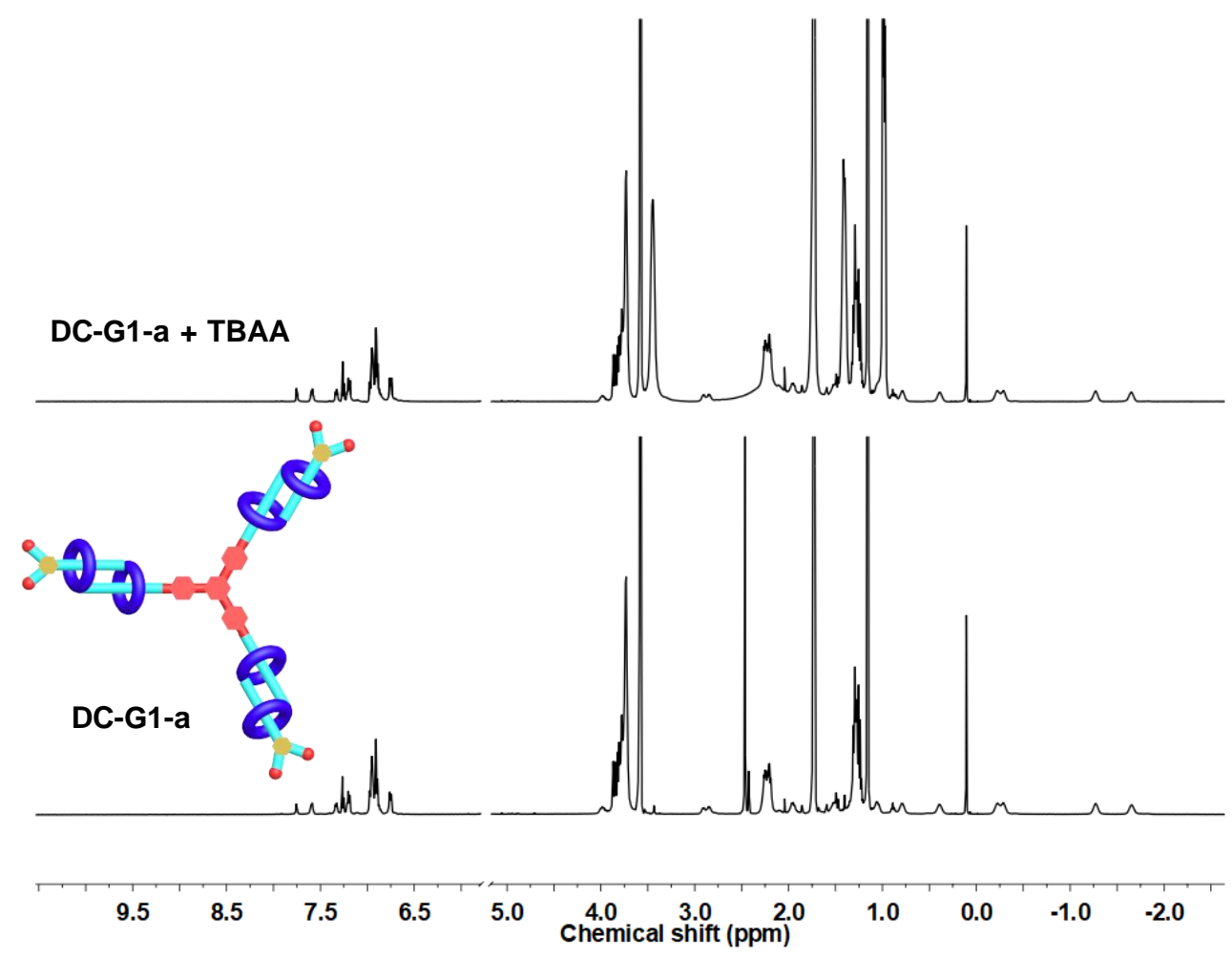

Figure S107: ${ }^{1} \mathrm{H}$ NMR spectra (THF- $d_{8}, 298 \mathrm{~K}, 500 \mathrm{MHz}$ ) of model dendrimer DC-G1-a with the addition of 30 eq. of TBAA (top) and model dendrimer DC-G1-a (bottom).

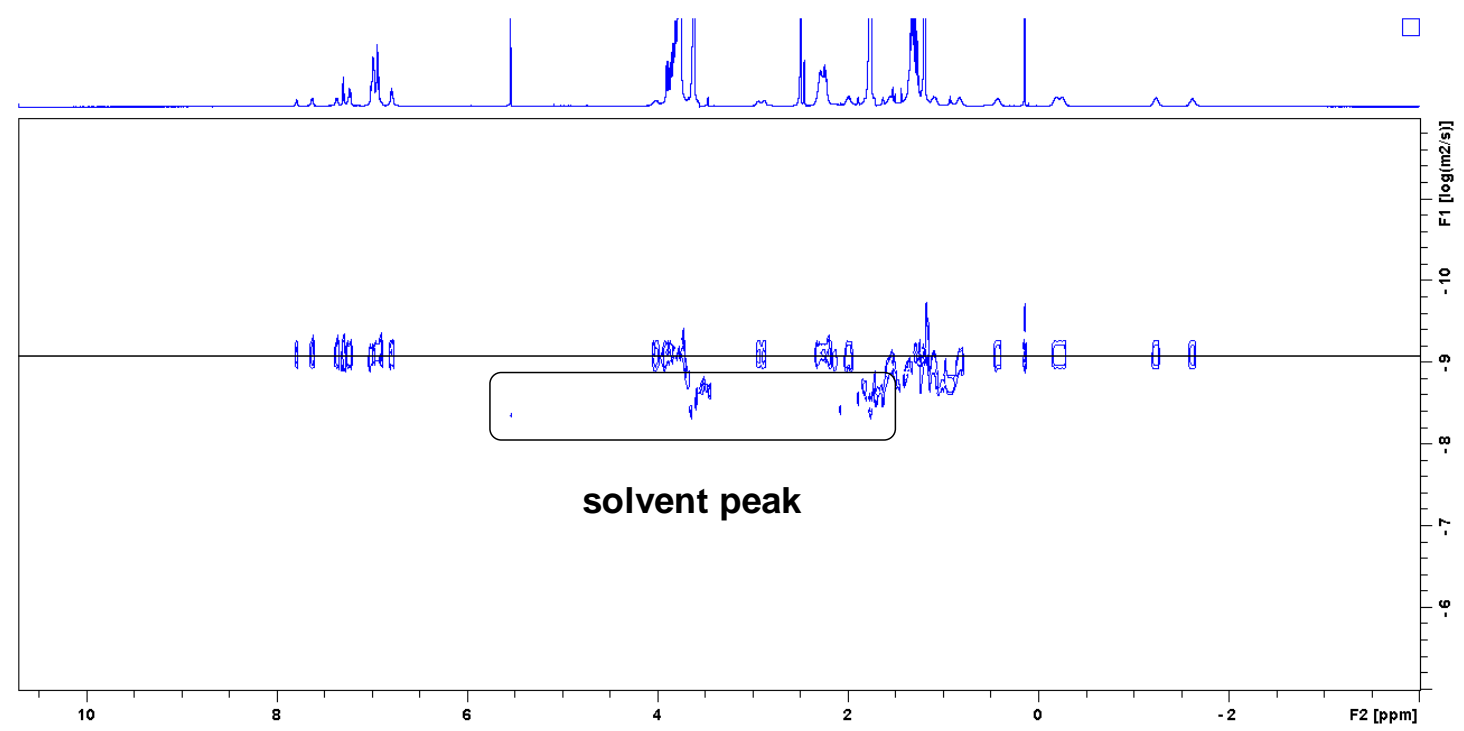

Figure S108: 2-D DOSY spectrum (THF- $d_{8}, 298 \mathrm{~K}, 500 \mathrm{MHz}$ ) of model daisy chain dendrimer DC-G1-a. 


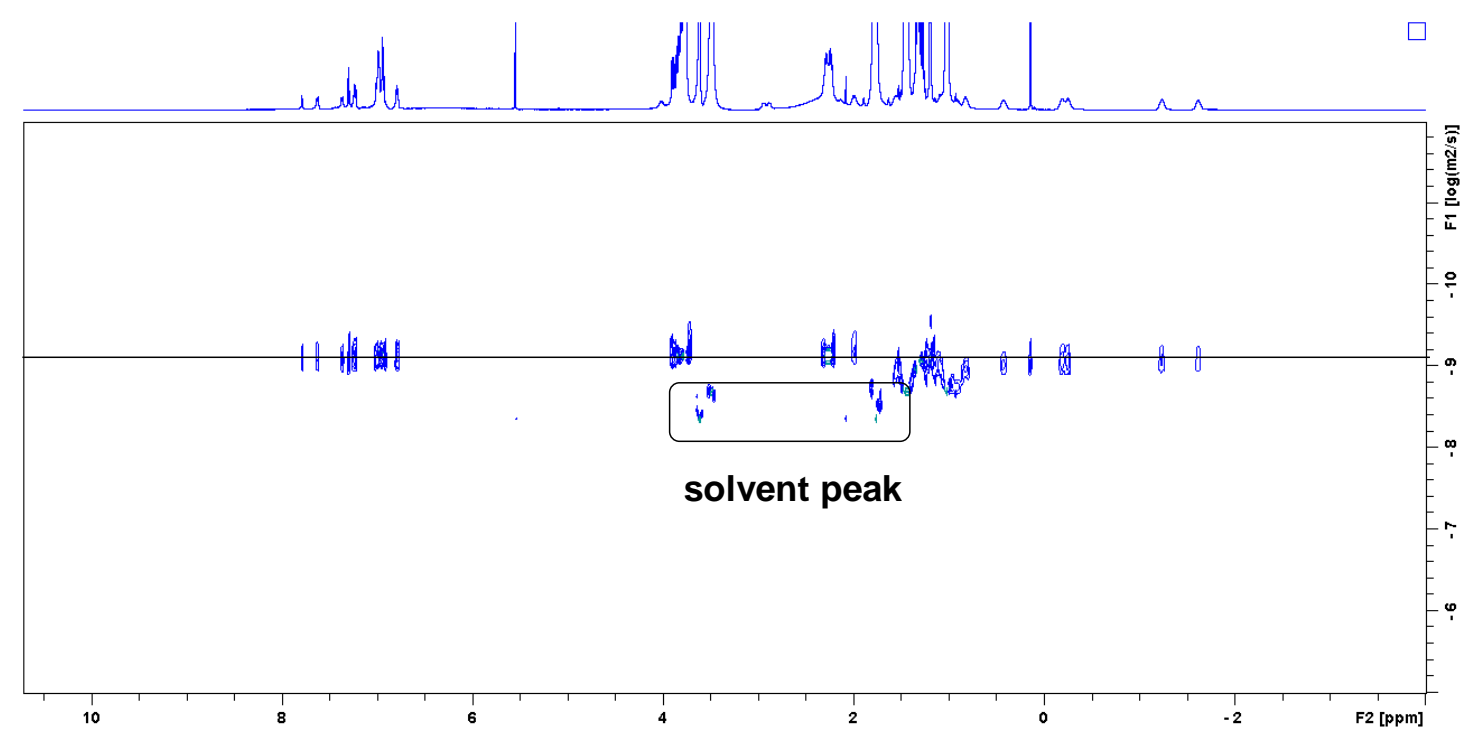

Figure S109: 2-D DOSY spectrum (THF- $d_{8}, 298 \mathrm{~K}, 500 \mathrm{MHz}$ ) of model daisy chain dendrimer DC-G1-a with the addition of 30.0 eq. of TBAA.

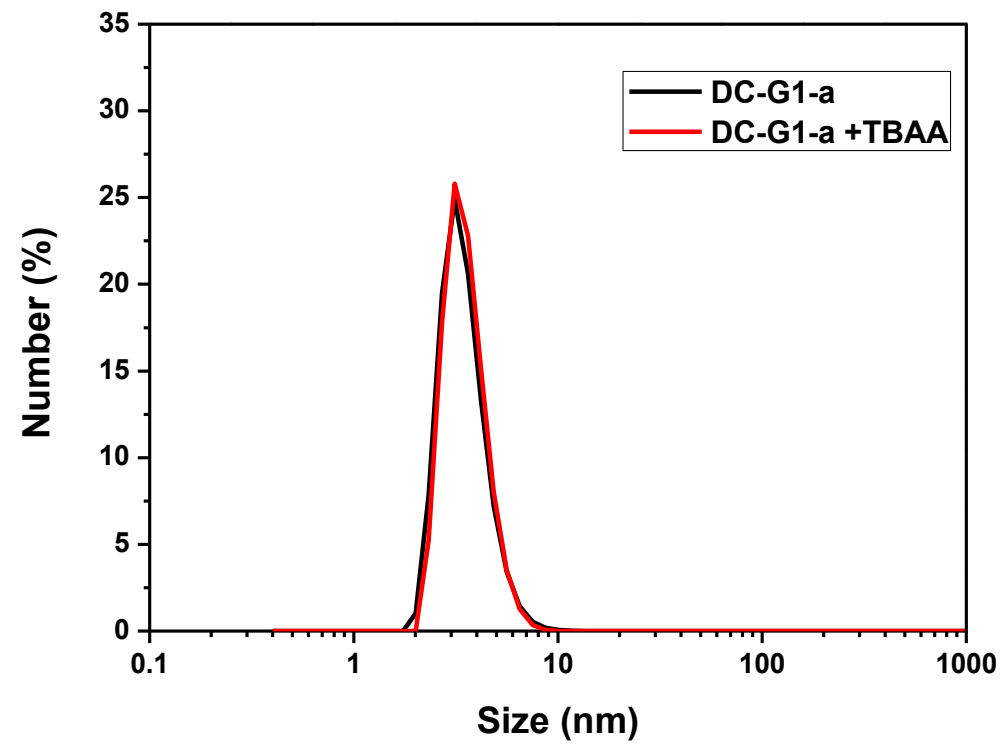

Figure S110: DLS spectra of model daisy chain dendrimer DC-G1-a $(3.5 \pm 0.1 \mathrm{~nm})$ and DC-G1-a with the addition of TBAA $(3.6 \pm 0.1 \mathrm{~nm})$. 


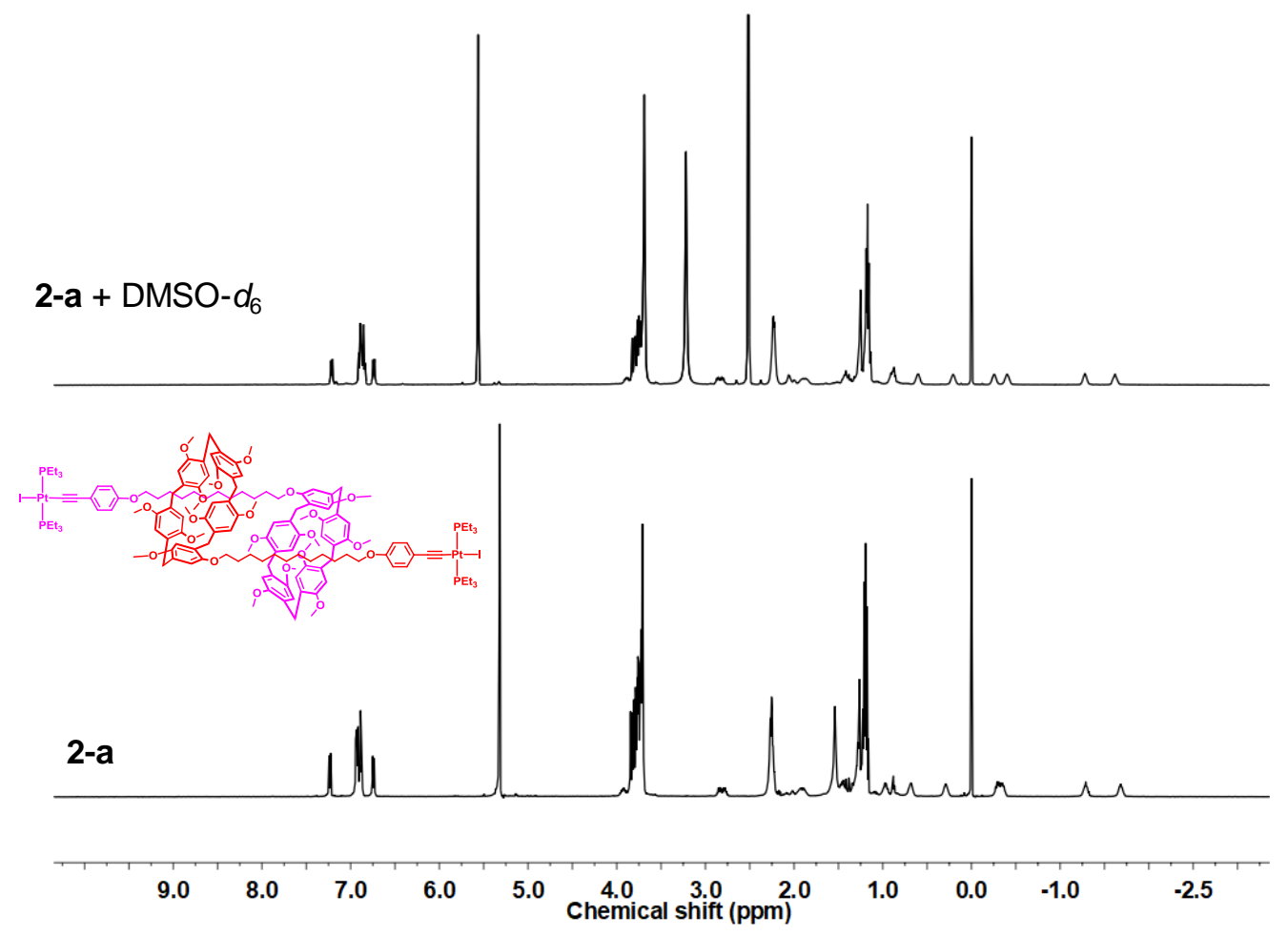

Figure S111: ${ }^{1} \mathrm{H}$ NMR spectra $\left(\mathrm{CD}_{2} \mathrm{Cl}_{2}(400 \mu \mathrm{L}), 298 \mathrm{~K}, 500 \mathrm{MHz}\right)$ of model complex 2-a (bottom); 2-a with the addition of $800 \mu \mathrm{L} \mathrm{DMSO}-d_{6}$ (top).

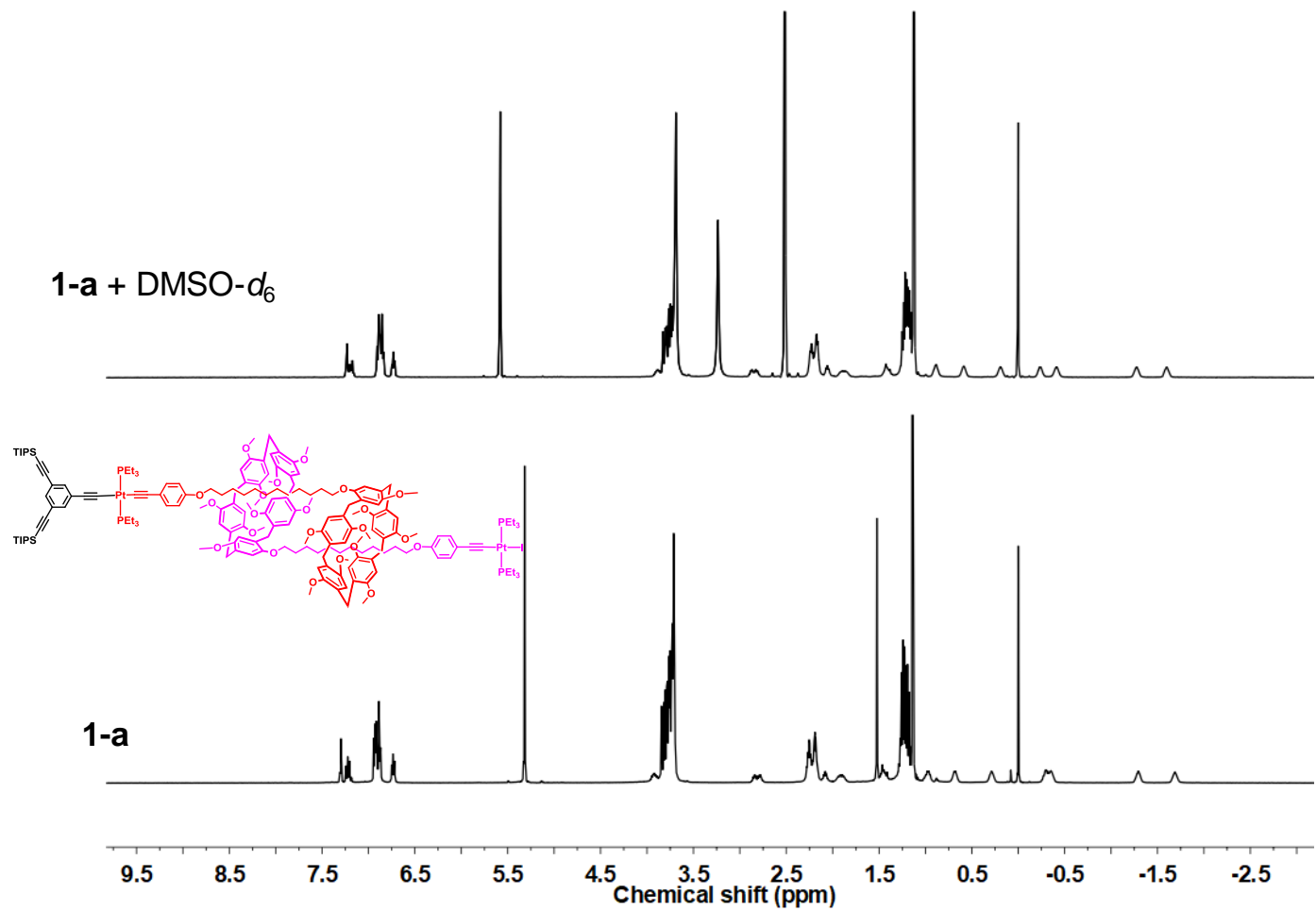

Figure S112: ${ }^{1} \mathrm{H}$ NMR spectra $\left(\mathrm{CD}_{2} \mathrm{Cl}_{2}(400 \mu \mathrm{L}), 298 \mathrm{~K}, 500 \mathrm{MHz}\right)$ of model complex 1-a (bottom); 1-a with the addition of $800 \mu \mathrm{L} \mathrm{DMSO}-d_{6}$ (top). 


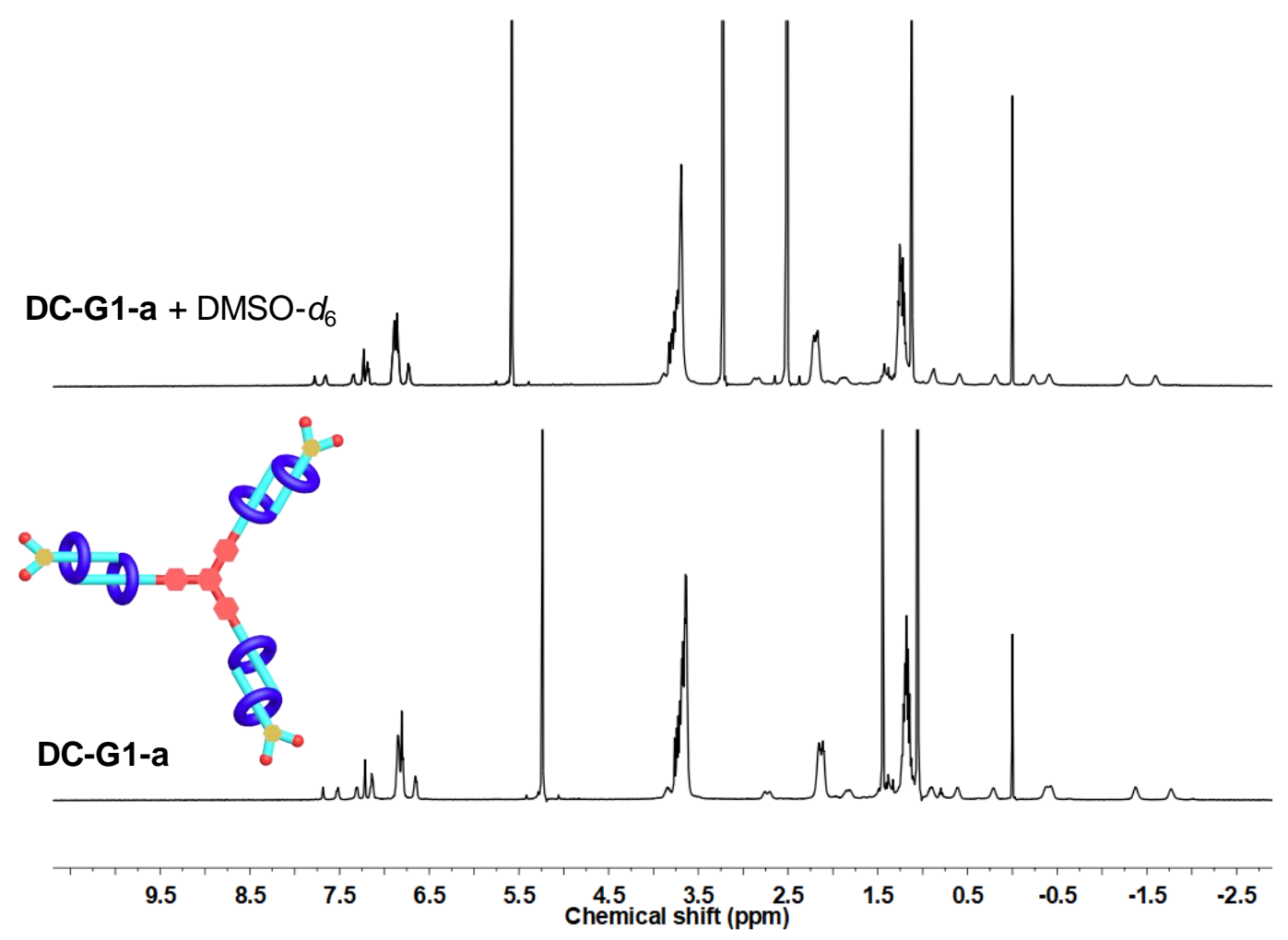

Figure S113: ${ }^{1} \mathrm{H}$ NMR spectra $\left(\mathrm{CD}_{2} \mathrm{Cl}_{2}(400 \mu \mathrm{L}), 298 \mathrm{~K}, 500 \mathrm{MHz}\right)$ of model dendrimer DC-G1-a (bottom); DC-G1-a with the addition of $800 \mu \mathrm{L} \mathrm{DMSO}-d_{6}$ (top).

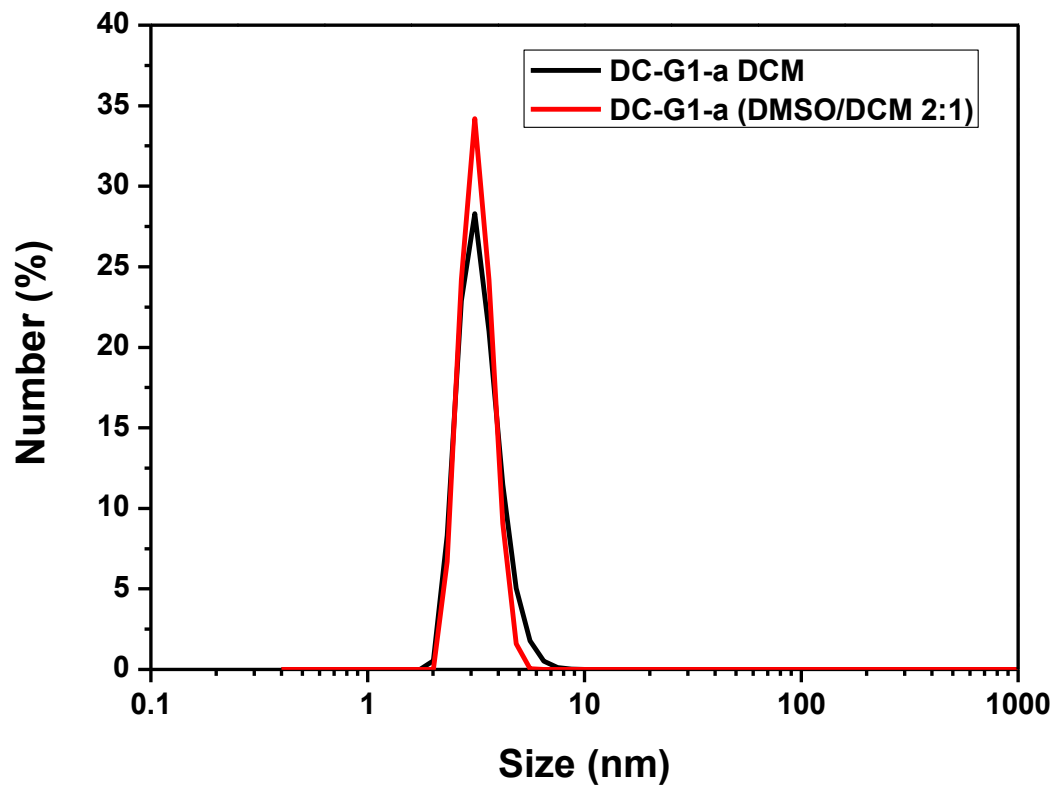

Figure S114: DLS spectra of model daisy chain dendrimer DC-G1-a $(3.3 \pm 0.1 \mathrm{~nm})$ and DC-G1-a in DMSO/DCM $(3.4 \pm 0.2 \mathrm{~nm})$. 


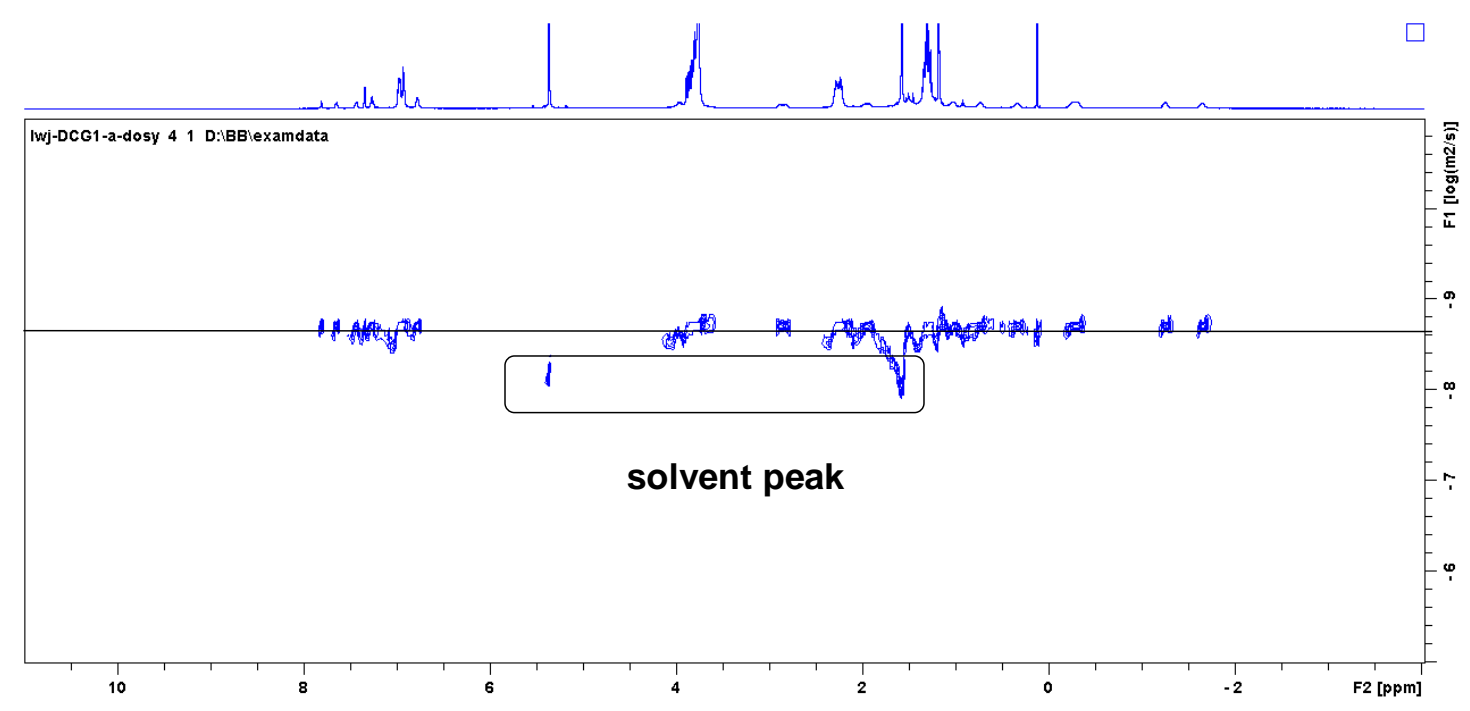

Figure S115: 2-D DOSY spectrum $\left(\mathrm{CD}_{2} \mathrm{Cl}_{2}, 298 \mathrm{~K}, 500 \mathrm{MHz}\right)$ of model daisy chain dendrimer DC-G1-a.

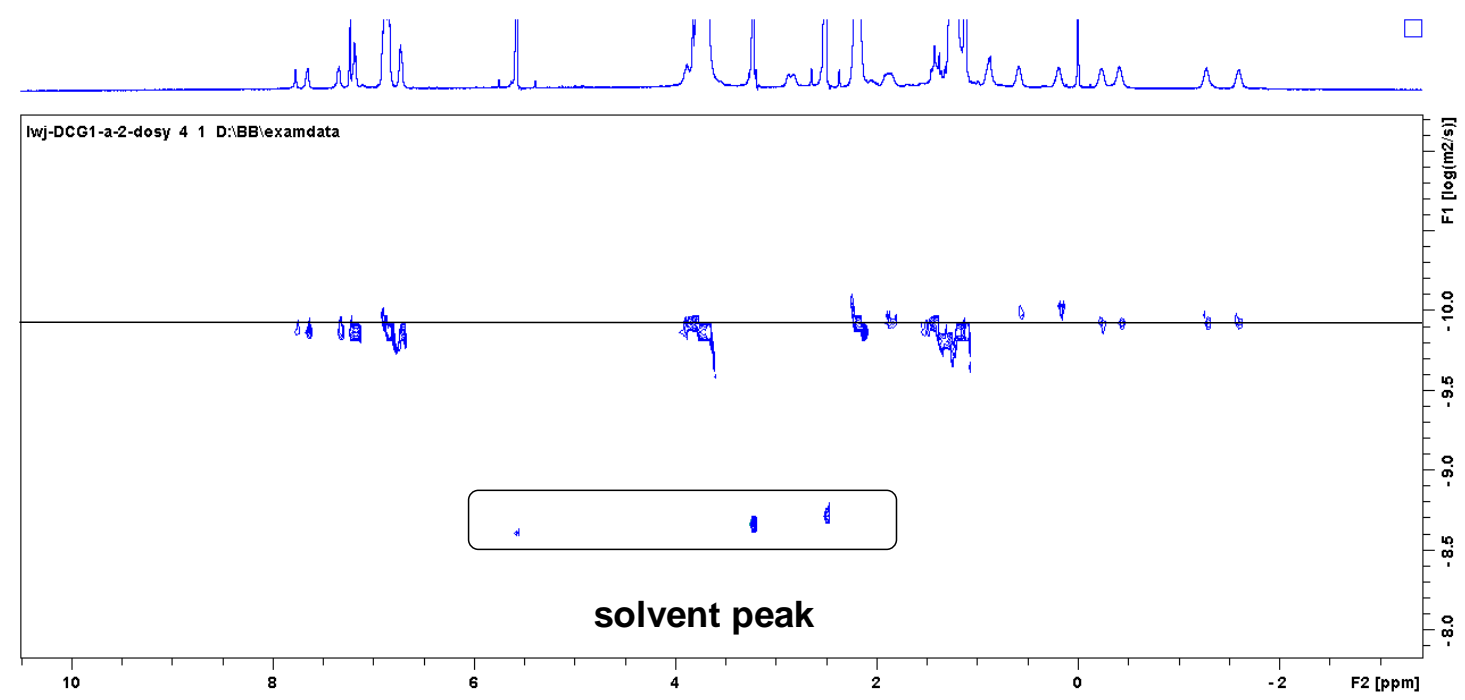

Figure S116: 2-D DOSY spectrum $\left(\mathrm{CD}_{2} \mathrm{Cl}_{2}, 298 \mathrm{~K}, 500 \mathrm{MHz}\right)$ of model daisy chain dendrimer DC-G1-a with the addition of DMSO- $d_{6}$. 
Section 7. Solvent-induced shape transformation behaviors of DC-Gn@PVDF films

Preparation of DC-Gn@PVDF Films. A DCM solution (200 $\mu \mathrm{L})$ of daisy chain dendrimers DC-Gn $(70 \mathrm{mg})$ was added into a DMF solution $(1.9 \mathrm{~mL})$ of the PVDF polymer $(130 \mathrm{mg})$ and the mixture was under ultrasonic treatment for $3 \mathrm{~h}$ to ensure that DC-Gn dispersed homogeneously, the resultant suspension was sequentially spread onto a glass substrate through drop-casting method followed by drying at $70{ }^{\circ} \mathrm{C}$ to give rise to DC-Gn@PVDF films. The DC-Gn@PVDF films were cut into roundness with $25 \mathrm{~mm}$ diameter (thickness $0.1 \mathrm{~mm}$ ). Notably, to help making the shape transformation processes more visible, Lissamine Rhodamine B was incorporated into the film.
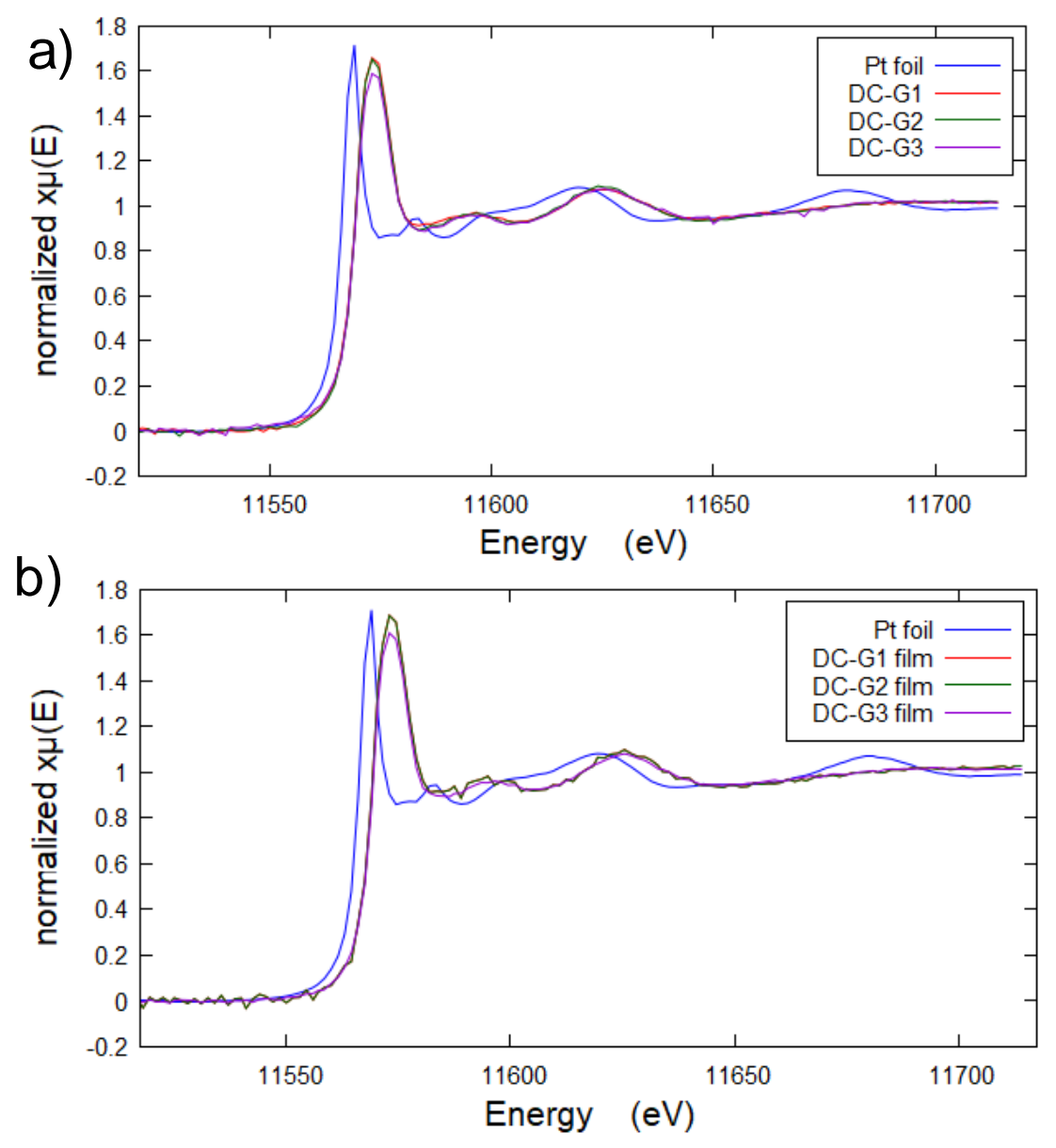

Figure S117: The X-ray absorption near-edge structure (XANES) spectra at the Pt $\mathrm{L}_{3}$-edge of a) daisy chain dendrimers DC-Gn and b) DC-Gn@PVDF films. XANES analysis demonstrated that the daisy chain dendrimers DC-Gn kept intact in the resultant DC-Gn@PVDF films. 

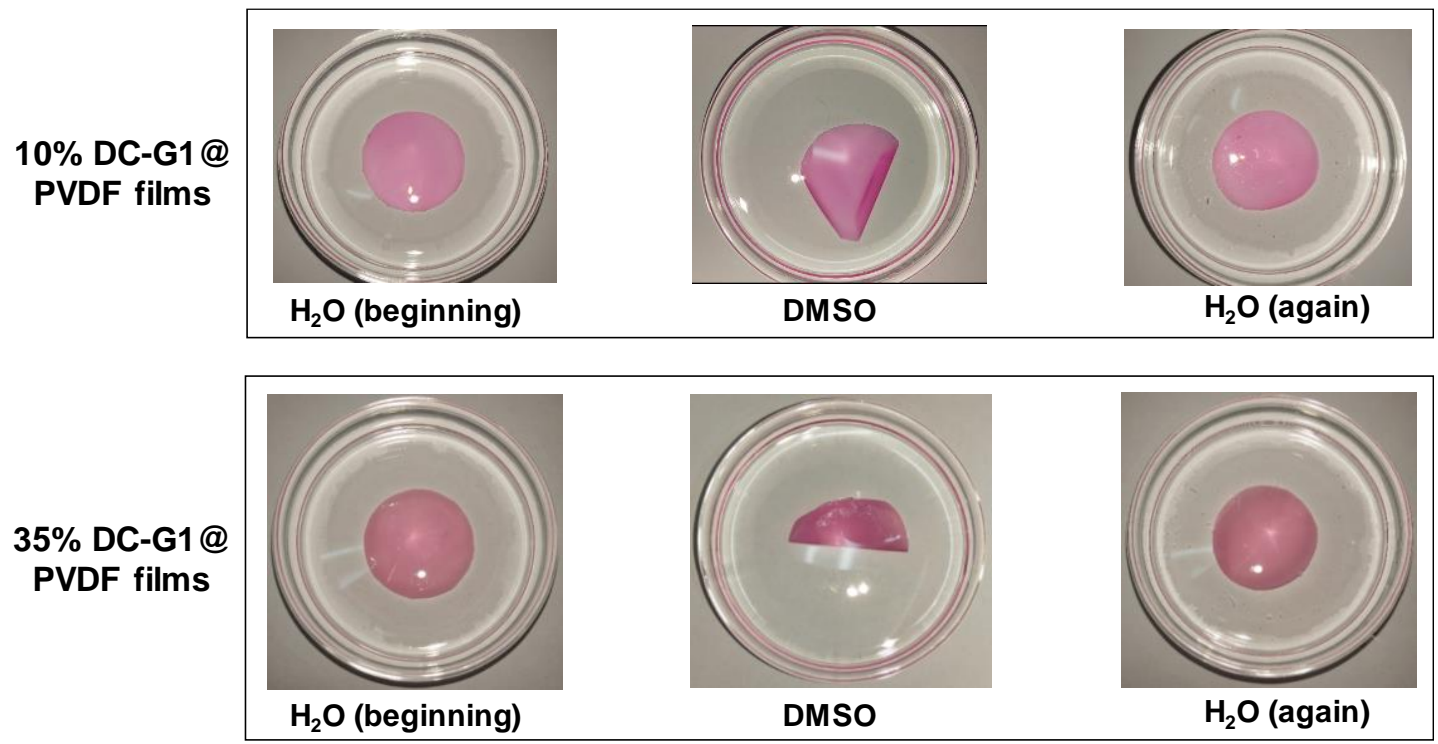

Figure S118: The different loading of DC-G1 (10 wt $\%$ and $35 \mathrm{wt} \%$ ) in PVDF films resulted in different shape transformation efficiency in DMSO.

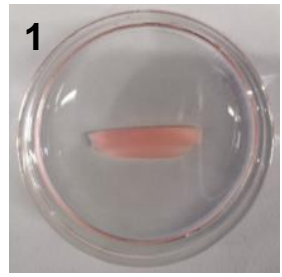

DMSO

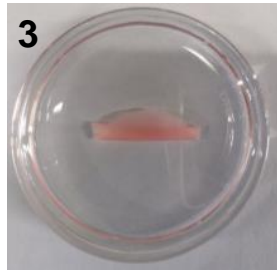

DMSO

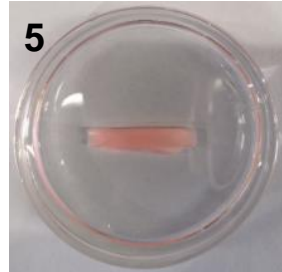

DMSO

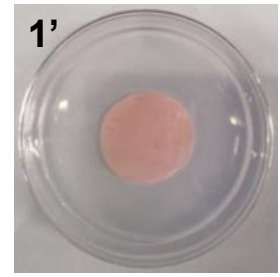

$\mathrm{H}_{2} \mathrm{O}$

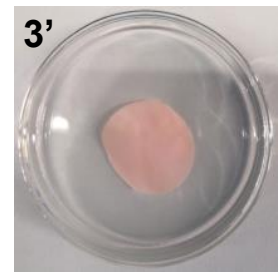

$\mathrm{H}_{2} \mathrm{O}$

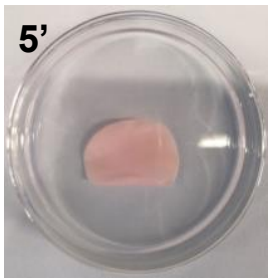

$\mathrm{H}_{2} \mathrm{O}$

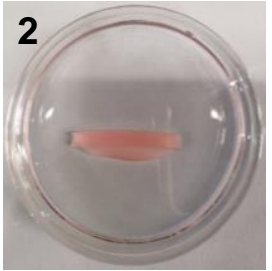

DMSO

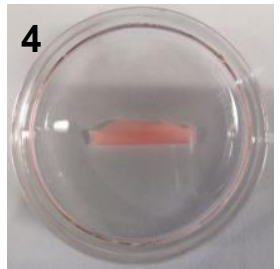

DMSO

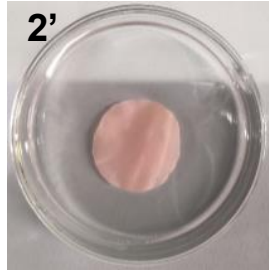

$\mathrm{H}_{2} \mathrm{O}$

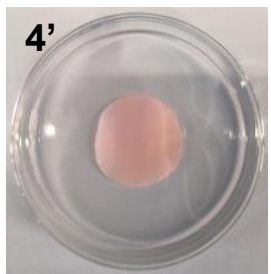

$\mathrm{H}_{2} \mathrm{O}$

Figure S119: Solvent-triggered reversible shape transformations of DC-G3@PVDF film for 5 times. 


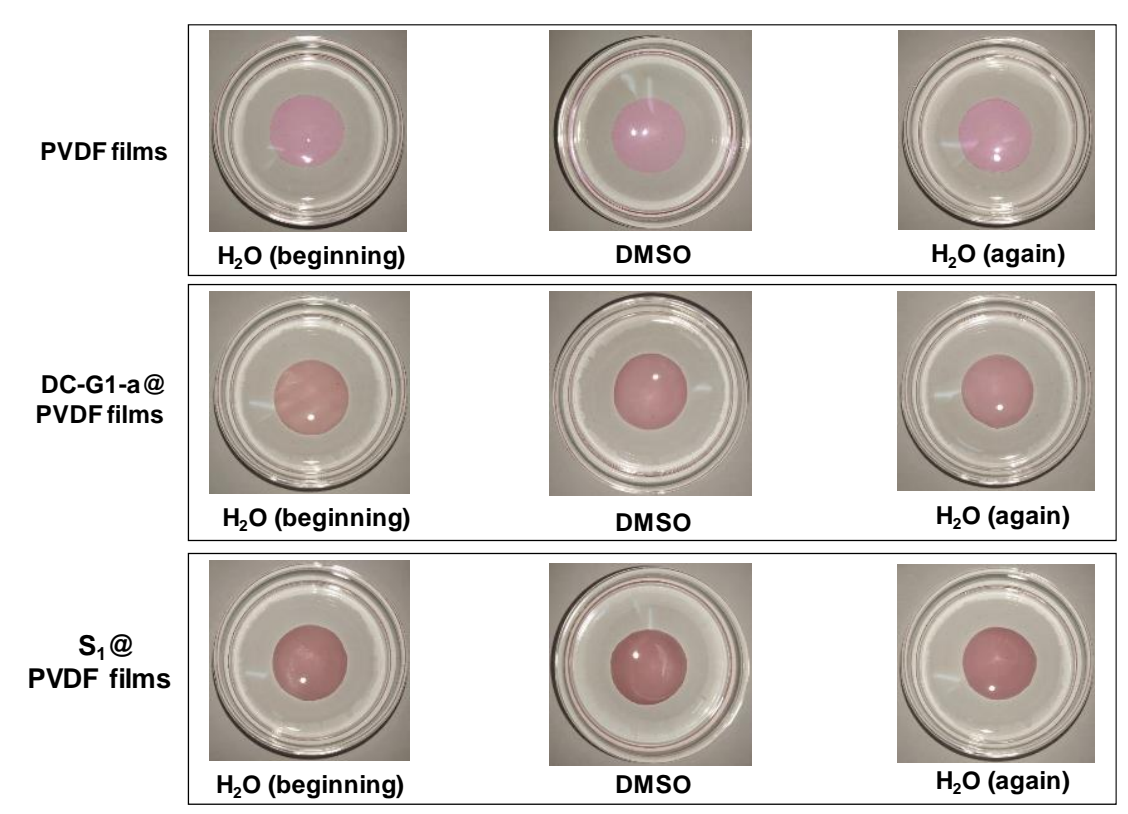

Figure S120: Control experiments of the shape transformation behaviors.

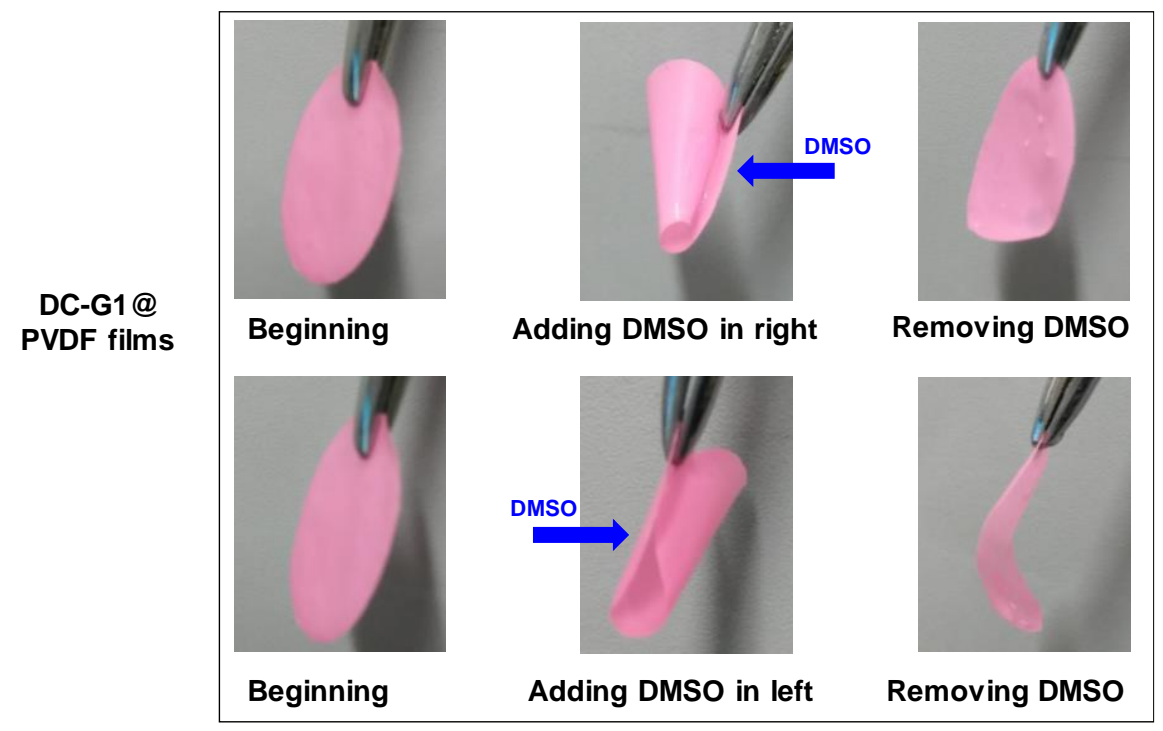

Figure S121: Controllable shape transformation of the DC-G1@PVDF films upon dropping DMSO solvent onto one side. The driving force of the controllable folding of the DC-G1@PVDF films was the reversible swelling/shrinking of DC-G1.

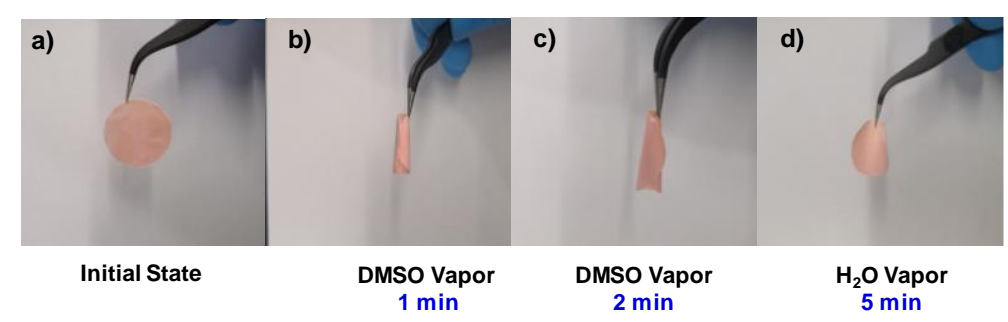

Figure S122: Vapor-triggered reversible shape transformations of DC-G3@PVDF film. 


\section{Section 8. Small angle neutron scattering (SANS) experiments}

In this work, SANS was performed on the Small Angle Neutron Scattering (SANS) instrument at China Spallation Neutron Source (CSNS) ${ }^{\text {S10 }}$. The incident neutrons with wavelength of 1-9 $\AA$ were defined by a double-disc bandwidth chopper, which is collimated to the sample by a pair of apertures. The experiment used the sample to detector distance of $4 \mathrm{~m}$ and a sample aperture of $6 \mathrm{~mm}$. The $1 \mathrm{~m}$ square detector array composed of 120 linear He-3 gas tubes with the diameter of $8 \mathrm{~mm}$, which covers the Q-range between $0.01 \AA^{-1}$ and $1 \AA^{-1}$. The presented data correspond to $\sim 120 \mathrm{~min}$ of data collection time for each sample (@50kW). Neutron data were corrected for background scattering (empty sample holder), transmission and detector efficiency, and set to absolute units.

The concentration of sample solutions was $10 \mathrm{mg} / \mathrm{mL}$ and placed in quartz cuvettes with $2 \mathrm{~mm}$ path length for all the measurements. DC-G3@PVDF film was placed at a sample holder for all the measurements. For each of the sample measurements, SANS measurements of corresponding solvents were also performed for background reduction. The measured scattering intensity was corrected for detector sensitivity, and placed on an absolute scale using a calibrated standard.

The solution SANS data were analyzed by Guinier fitting. The low q regions of the data are fitted with Guinier's law, $I(q)=I_{0} \exp \left(-\frac{q^{2} R_{g}^{2}}{3}\right)$, and radii of gyration $\left(R_{\mathrm{g}} \mathrm{s}\right)$ can be obtained. For the film SANS data, Guinier's law with a power law background, $I(q)=$ scale $1 \times q^{-\alpha}+$ scale $2 \times \exp \left(-\frac{q^{2} R_{g}^{2}}{3}\right)+$ incoherent Background, was applied and radii of gyration $\left(R_{\mathrm{g} s}\right)$ can also be obtained. 
Section 9. Supplementary data

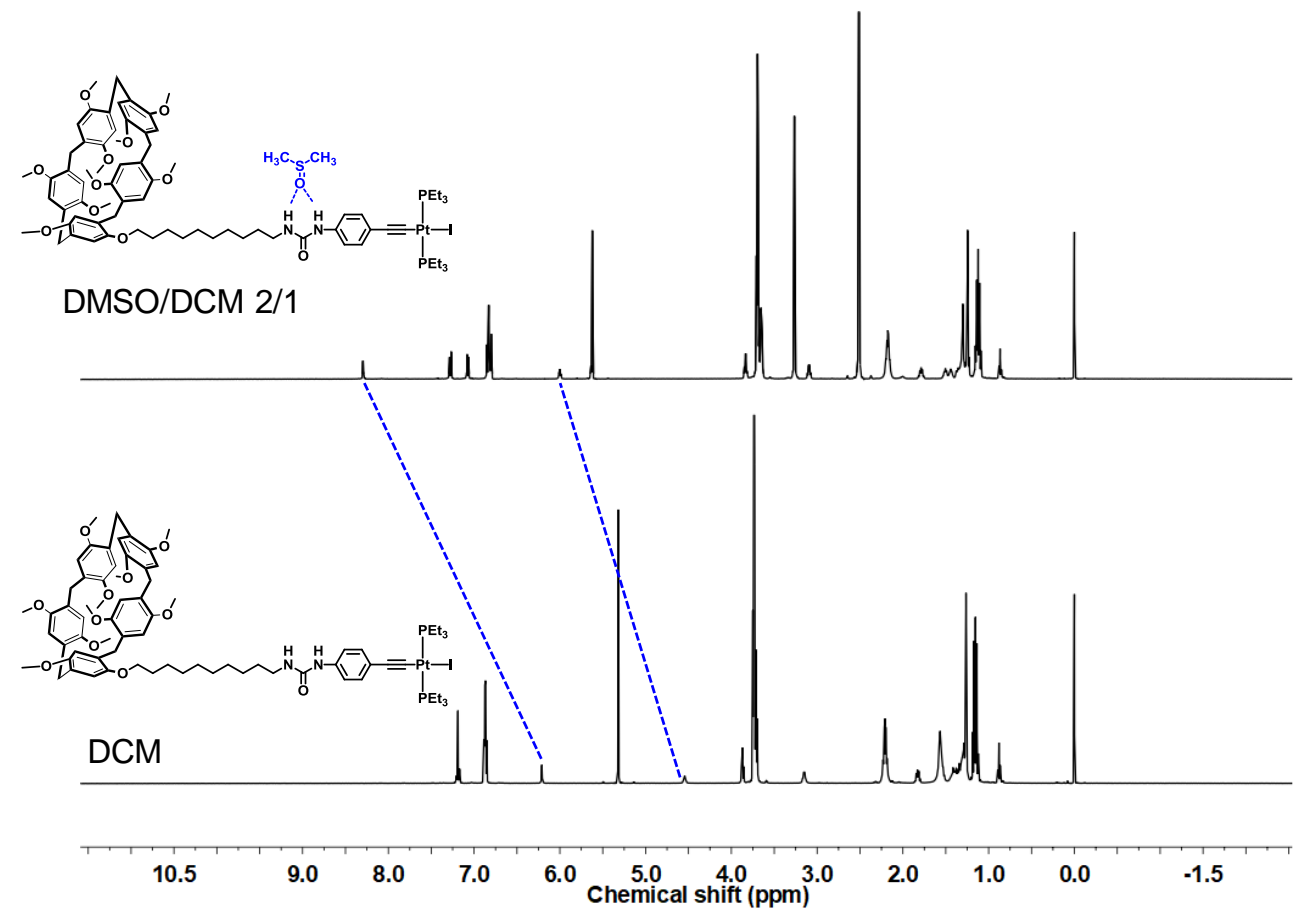

Figure S123: ${ }^{1} \mathrm{H}$ NMR spectra $\left(\mathrm{CD}_{2} \mathrm{Cl}_{2}(400 \mu \mathrm{L}), 298 \mathrm{~K}, 500 \mathrm{MHz}\right)$ of the compound $\mathrm{S}_{1}$ (bottom) and the addition of $800 \mu \mathrm{L}$ DMSO- $d_{6}$ (top).

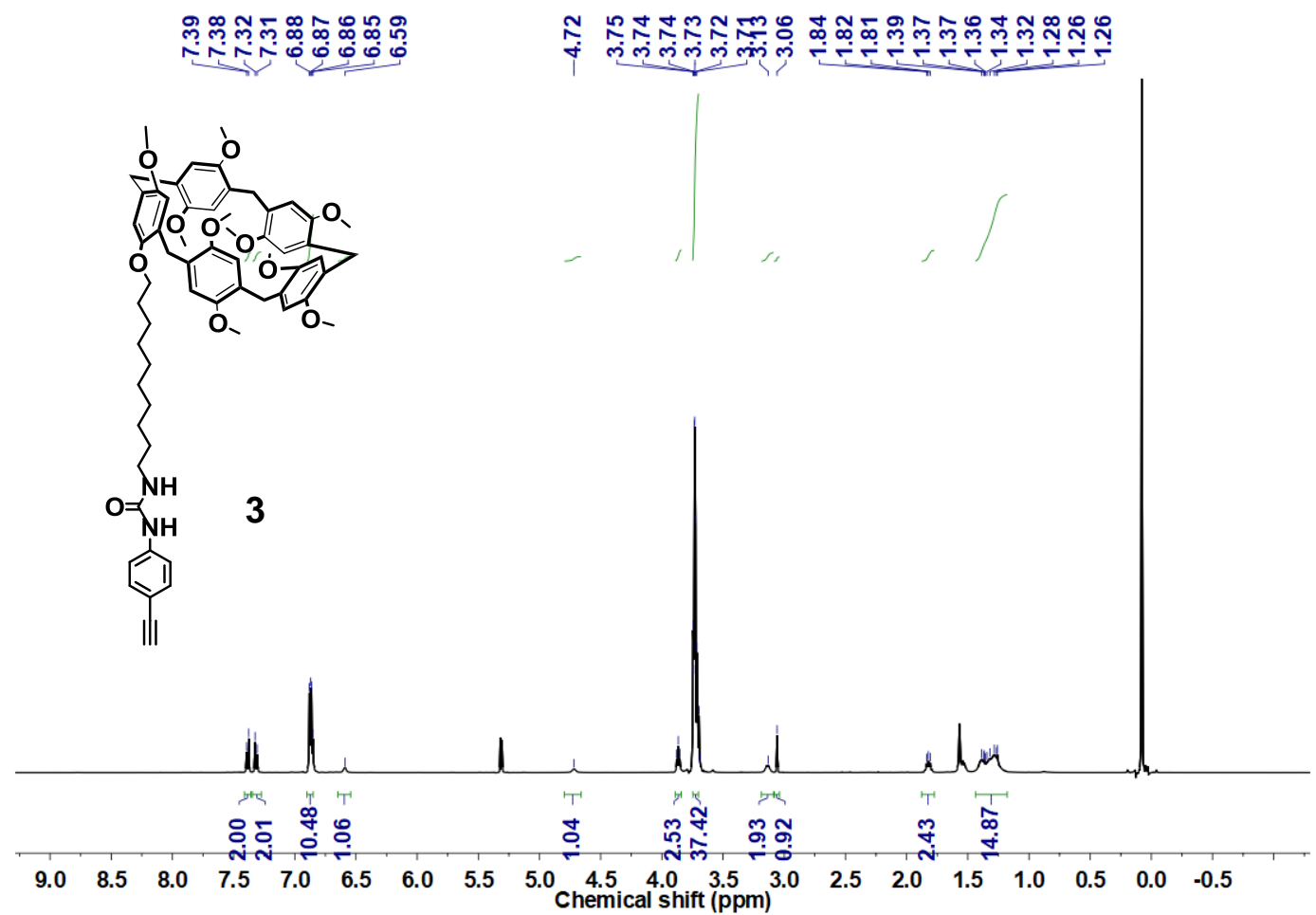

Figure S124: ${ }^{1} \mathrm{H}$ NMR spectrum $\left(\mathrm{CD}_{2} \mathrm{Cl}_{2}, 298 \mathrm{~K}, 500 \mathrm{MHz}\right)$ of the compound 3. 


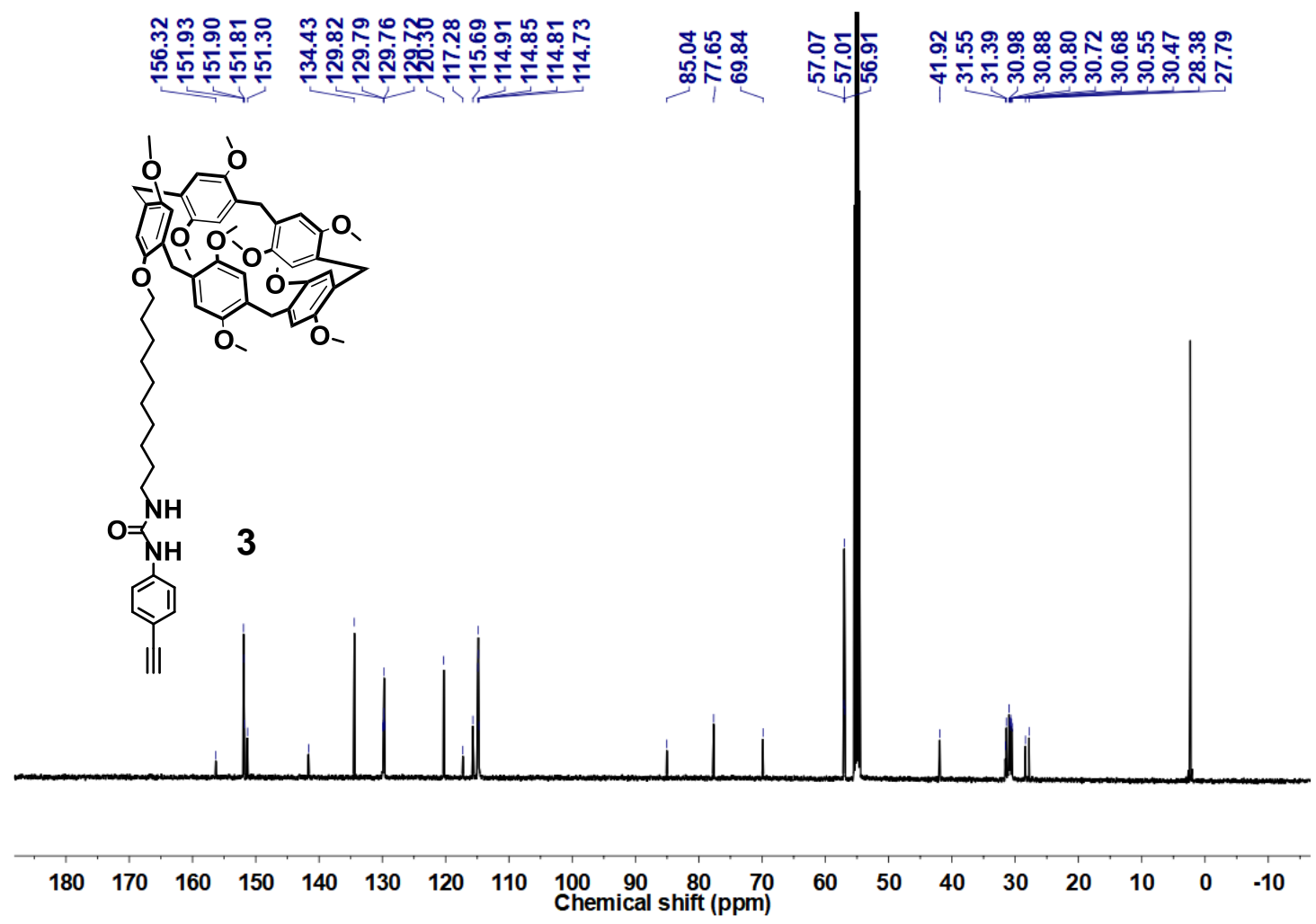

Figure S125: ${ }^{13} \mathrm{C}$ NMR spectrum $\left(\mathrm{CD}_{2} \mathrm{Cl}_{2}, 298 \mathrm{~K}, 126 \mathrm{MHz}\right)$ of the compound 3 .

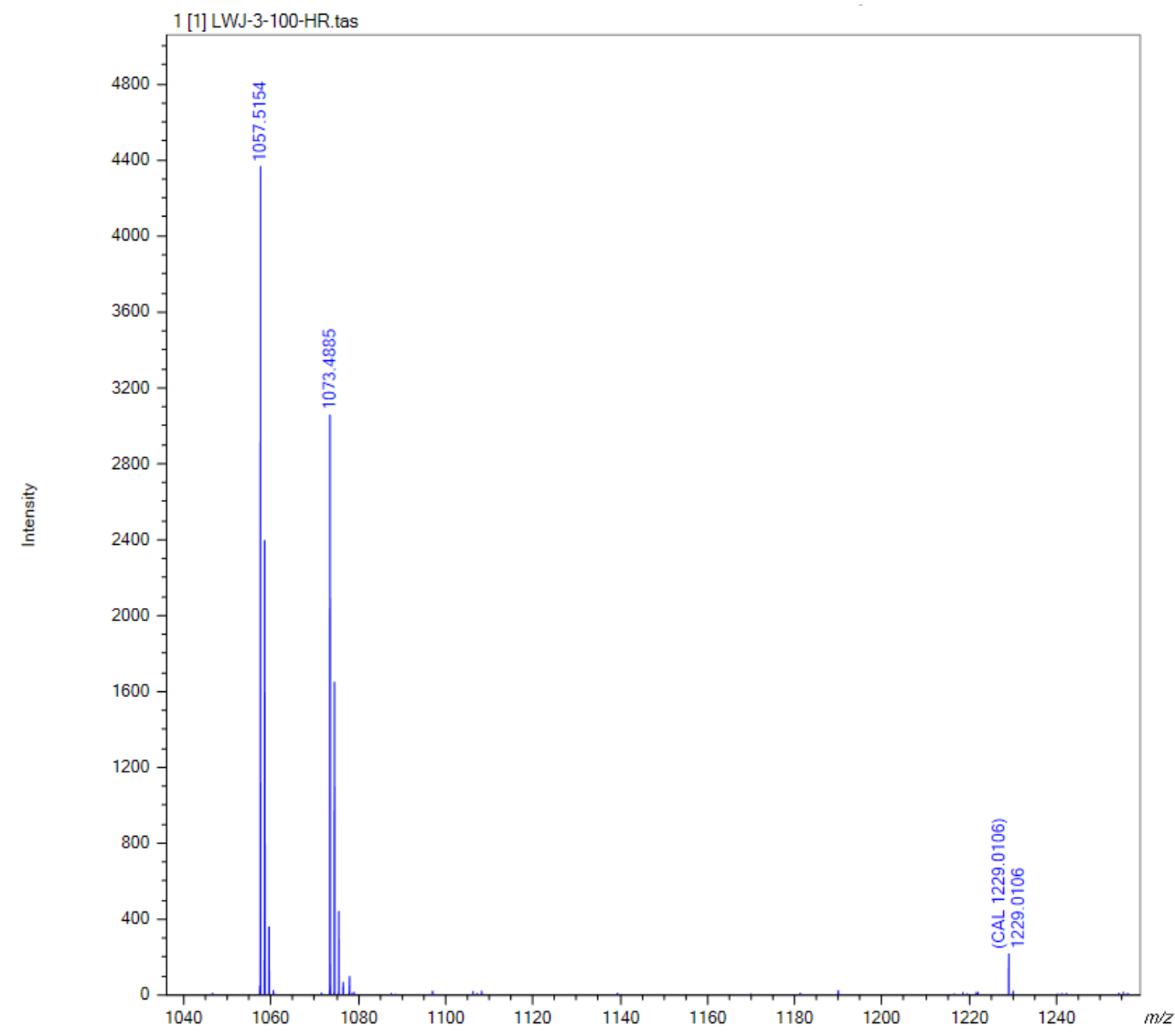

Figure S126: MALDI-TOF-MS spectrum of the compound 3. 
ำ

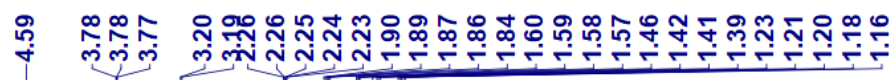

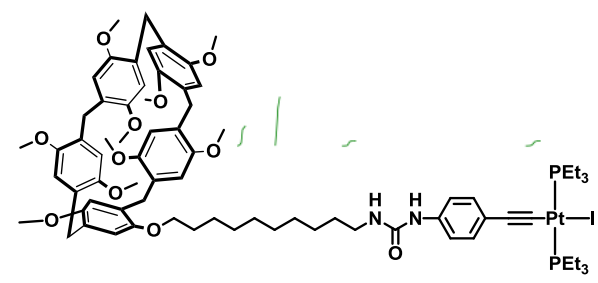

$\mathrm{S}_{1}$

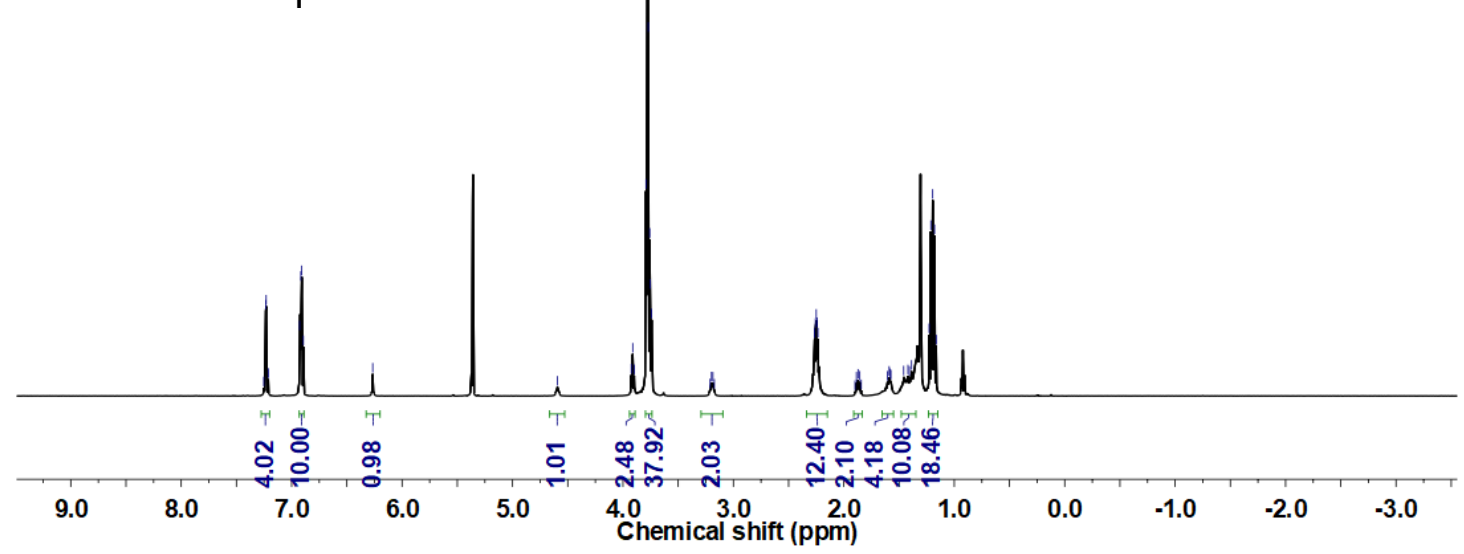

Figure S127: ${ }^{1} \mathrm{H}$ NMR spectrum $\left(\mathrm{CD}_{2} \mathrm{Cl}_{2}, 298 \mathrm{~K}, 500 \mathrm{MHz}\right)$ of the compound $\mathbf{S}_{\mathbf{1}}$.
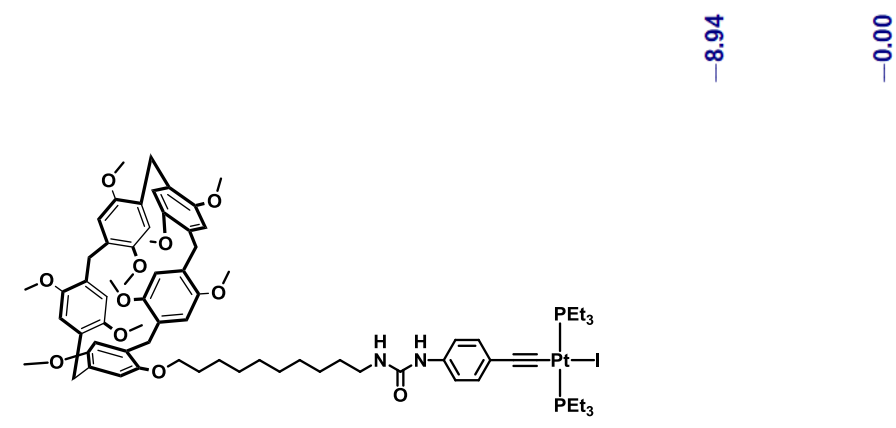

$S_{1}$
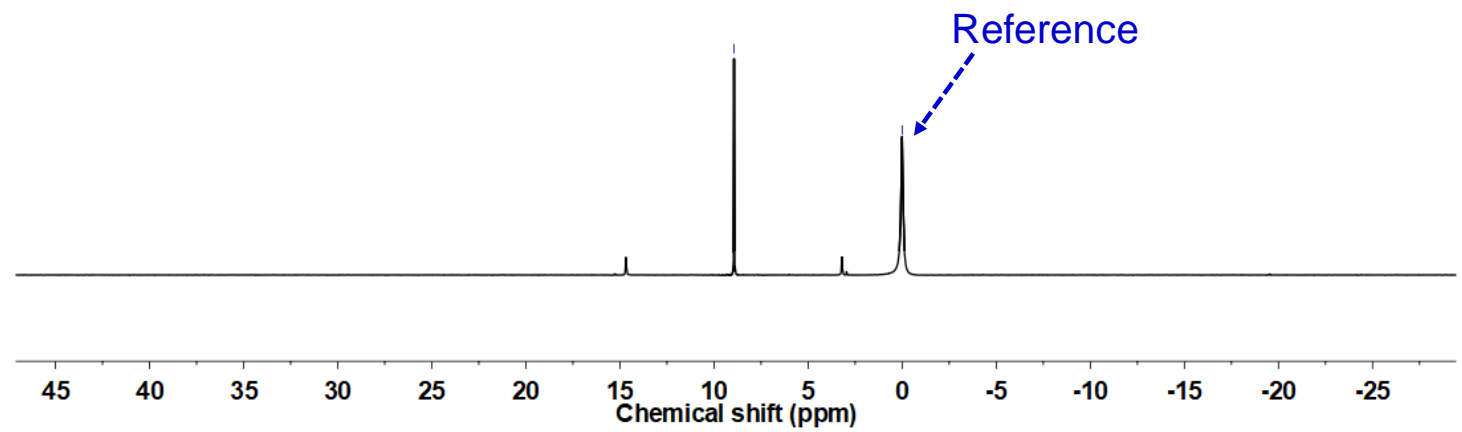

Figure S128: ${ }^{31} \mathrm{P}$ NMR spectrum $\left(\mathrm{CD}_{2} \mathrm{Cl}_{2}, 298 \mathrm{~K}, 202 \mathrm{MHz}\right)$ of the compound $\mathbf{S}_{\mathbf{1}}$. 


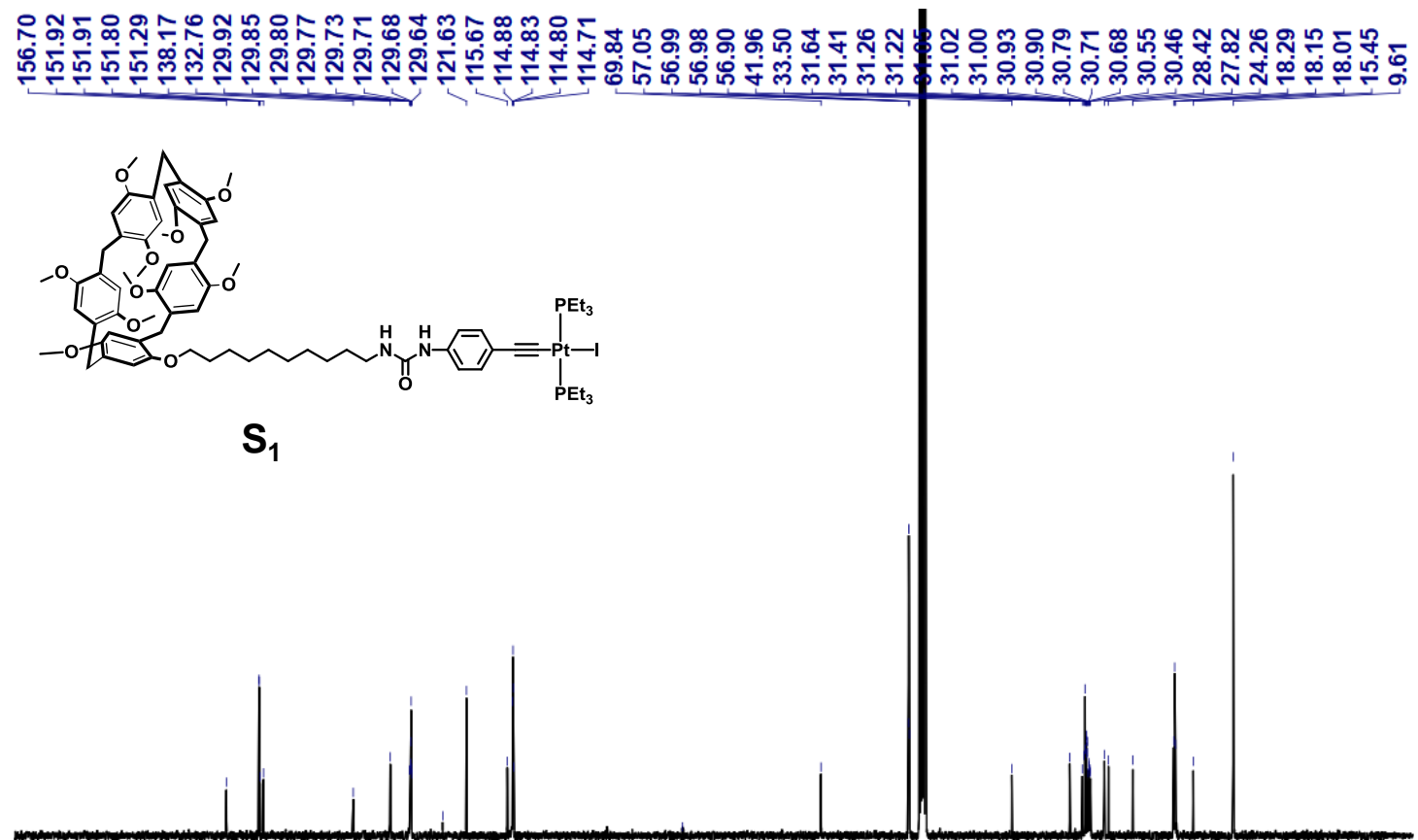

$\begin{array}{llllllllllllllllllll}180 & 170 & 160 & 150 & 140 & 130 & 120 & 110 & 100 & 90 & 80 & 70 & 60 & 50 & 40 & 30 & 20 & 10 & 0 & -10\end{array}$

Figure S129: ${ }^{13} \mathrm{C}$ NMR spectrum $\left(\mathrm{CD}_{2} \mathrm{Cl}_{2}, 298 \mathrm{~K}, 126 \mathrm{MHz}\right)$ of the compound $\mathbf{S}_{\mathbf{1}}$.

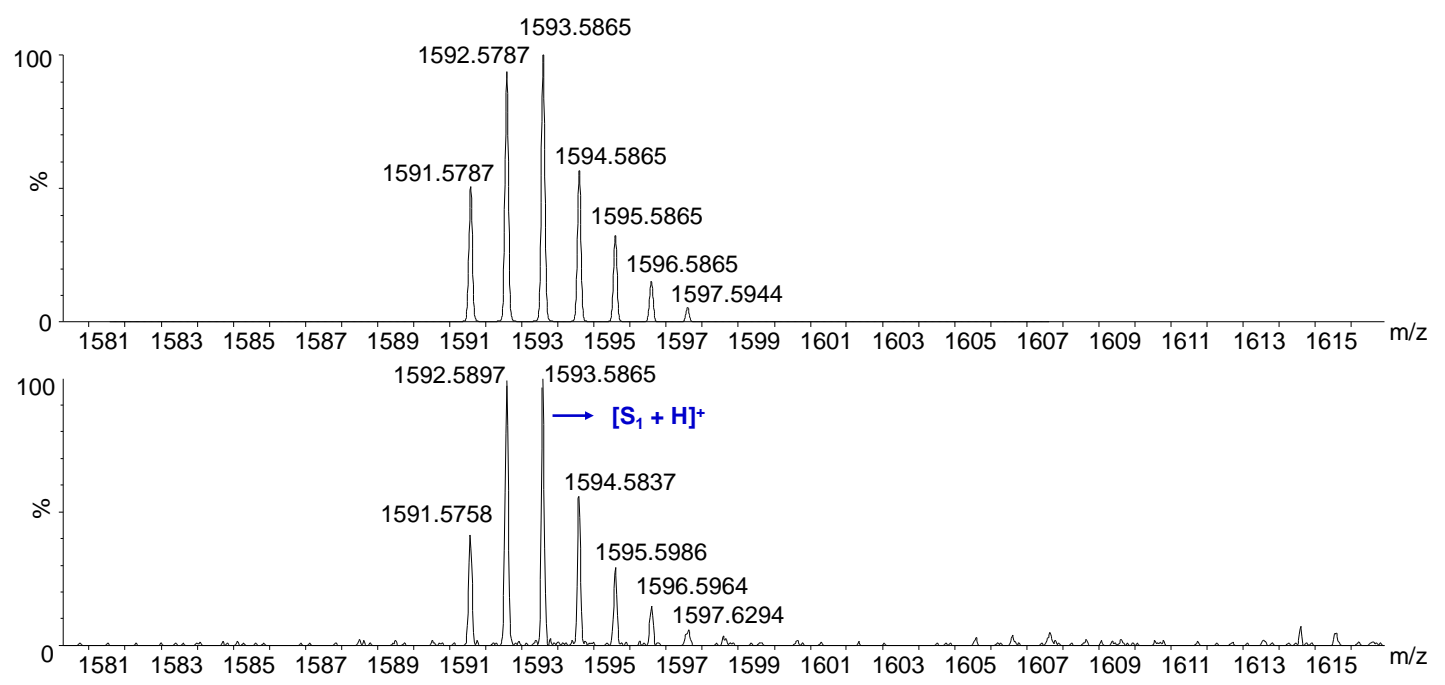

Figure S130: Theoretical (top) and experimental (bottom) ESI-TOF-MS spectra of the compound $\mathbf{S}_{\mathbf{1}}$. 


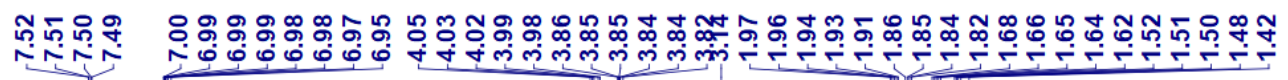

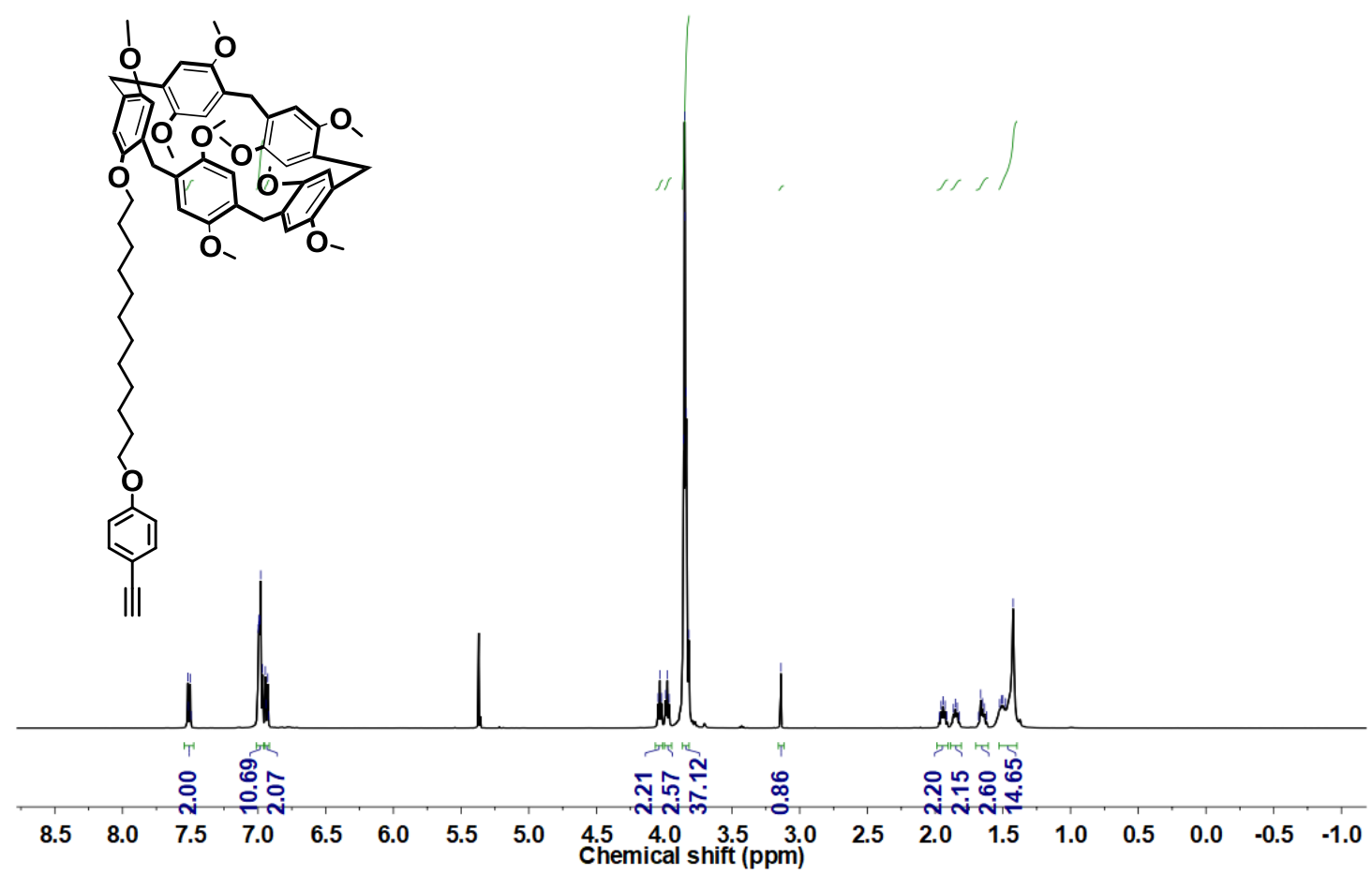

Figure S131: ${ }^{1} \mathrm{H}$ NMR spectrum $\left(\mathrm{CD}_{2} \mathrm{Cl}_{2}, 298 \mathrm{~K}, 500 \mathrm{MHz}\right)$ of model compound $\mathbf{S}$.

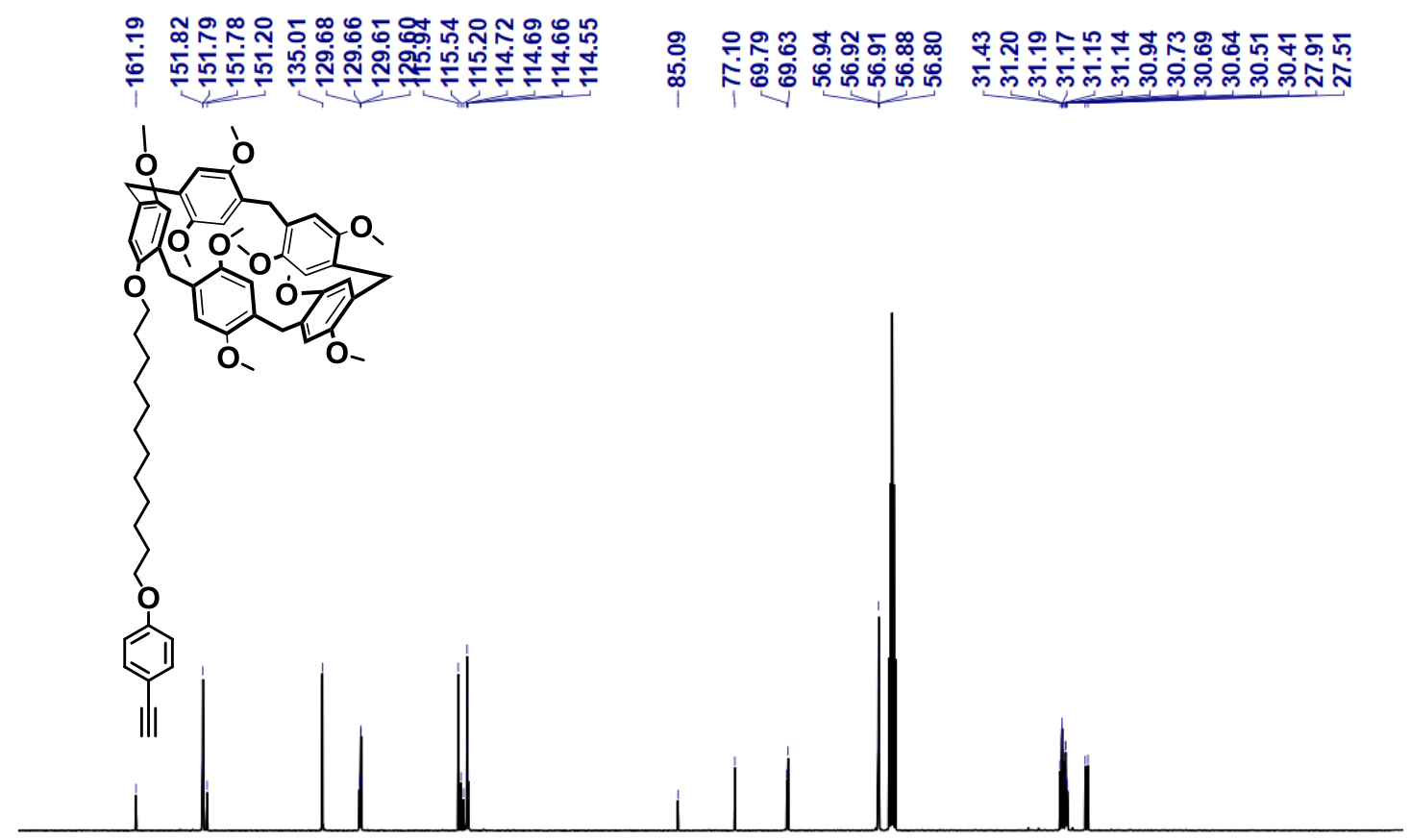

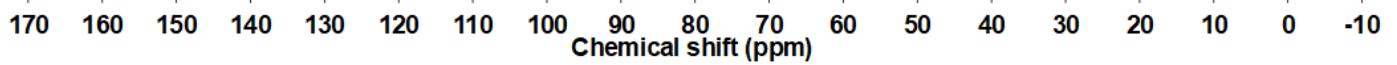

Figure S132: ${ }^{13} \mathrm{C}$ NMR spectrum $\left(\mathrm{CD}_{2} \mathrm{Cl}_{2}, 298 \mathrm{~K}, 126 \mathrm{MHz}\right)$ of model compound $\mathbf{S}$. 


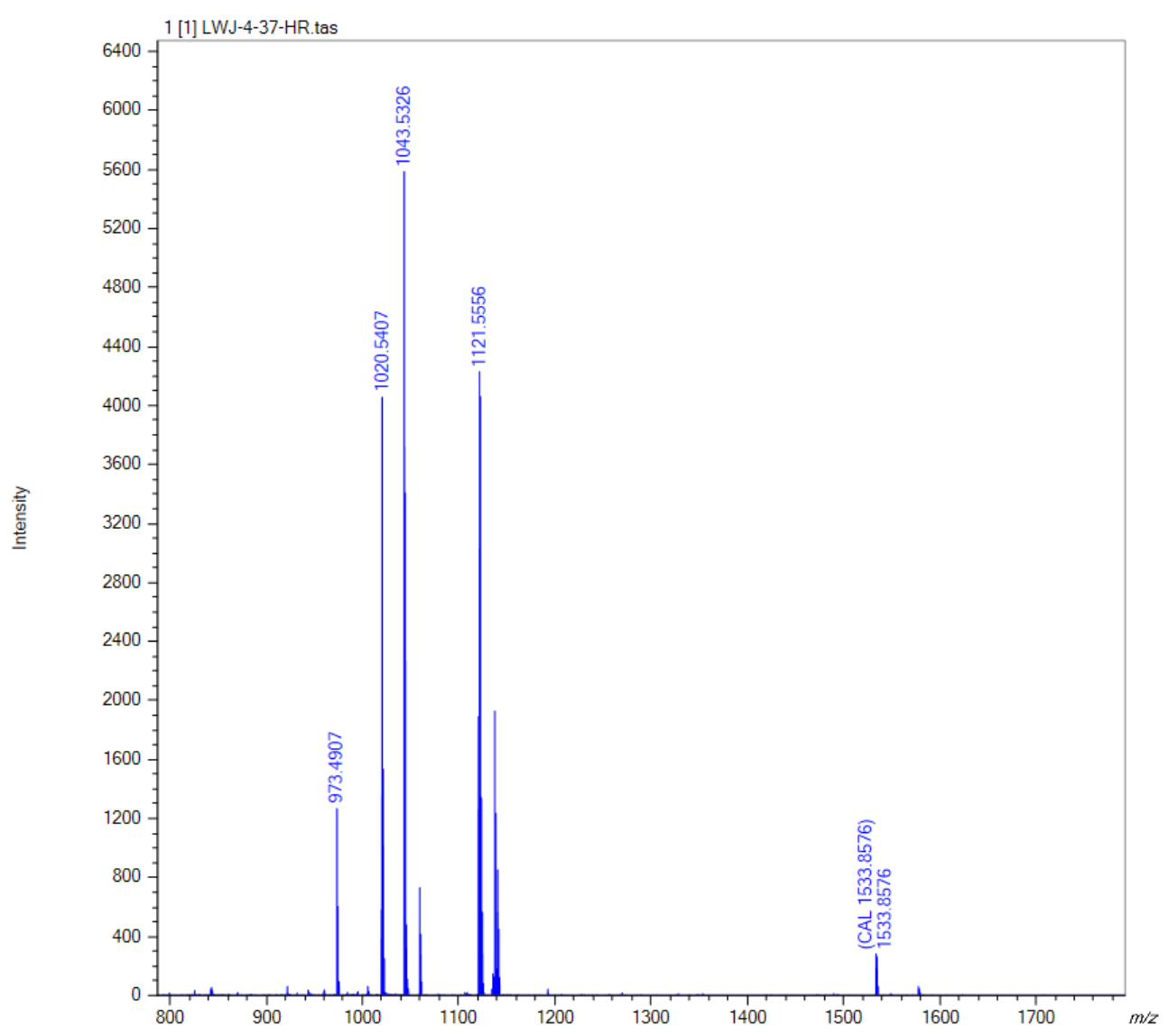

Figure S133: MALDI-TOF-MS spectrum of model compound S9.

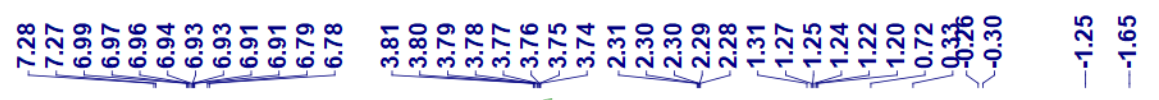
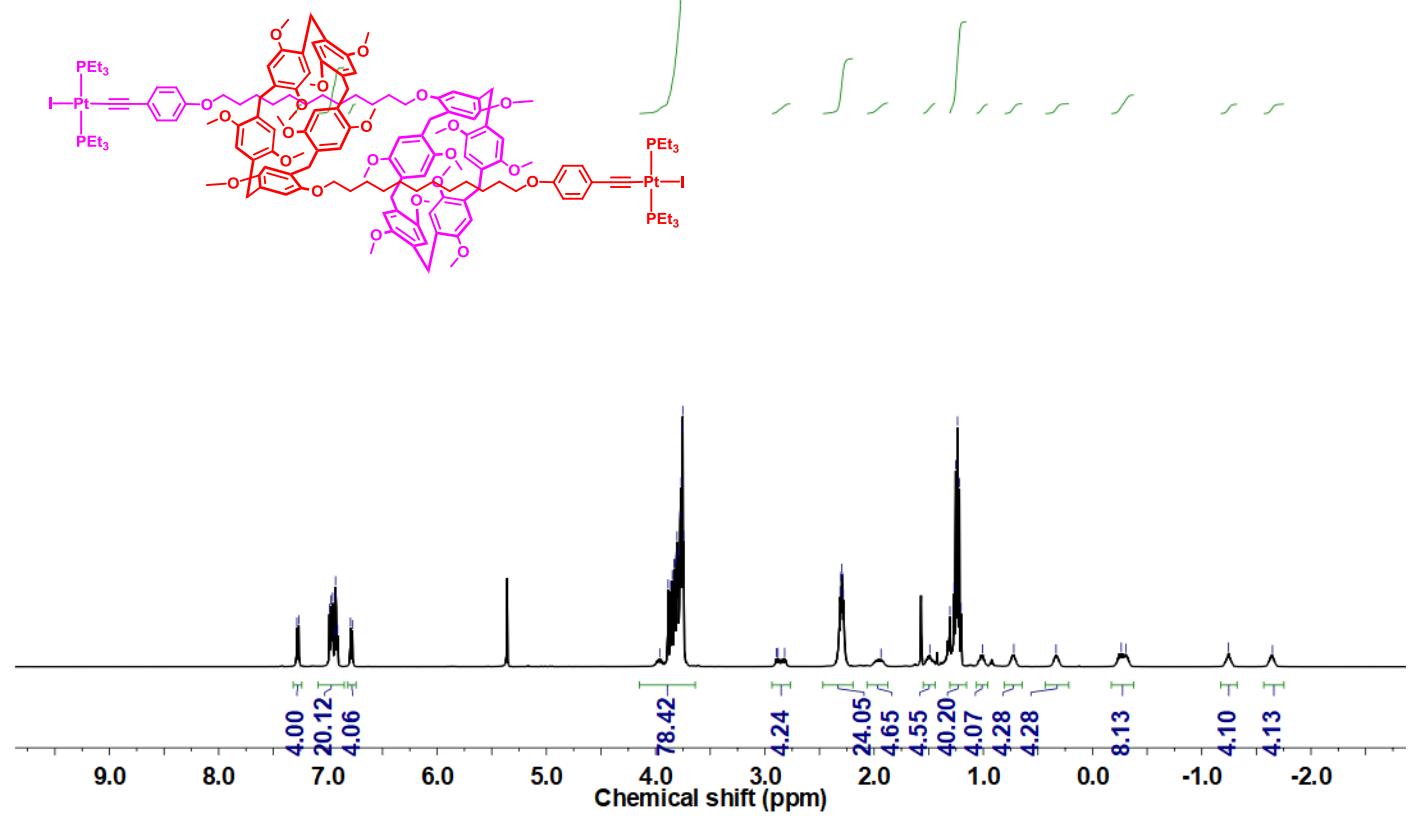

Figure S134: ${ }^{1} \mathrm{H}$ NMR spectrum $\left(\mathrm{CD}_{2} \mathrm{Cl}_{2}, 298 \mathrm{~K}, 500 \mathrm{MHz}\right)$ of model compound 2-a. 

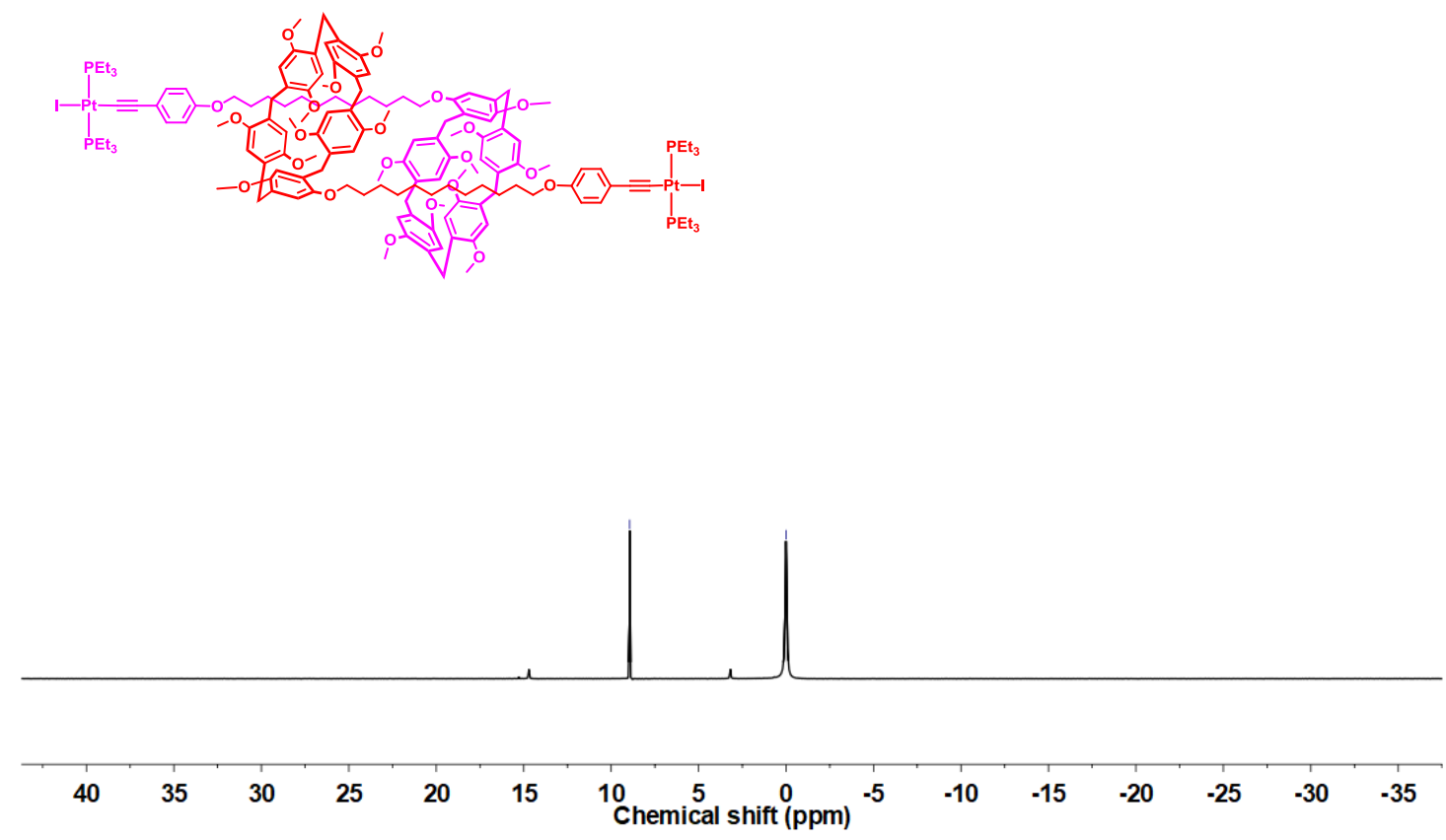

Figure S135: ${ }^{31} \mathrm{P}$ NMR spectrum $\left(\mathrm{CD}_{2} \mathrm{Cl}_{2}, 298 \mathrm{~K}, 202 \mathrm{MHz}\right)$ of model compound 2-a.

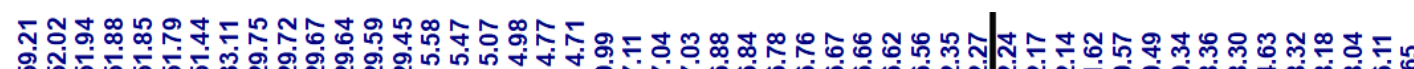

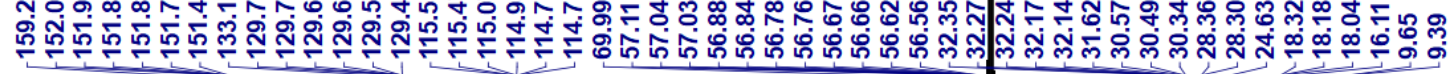
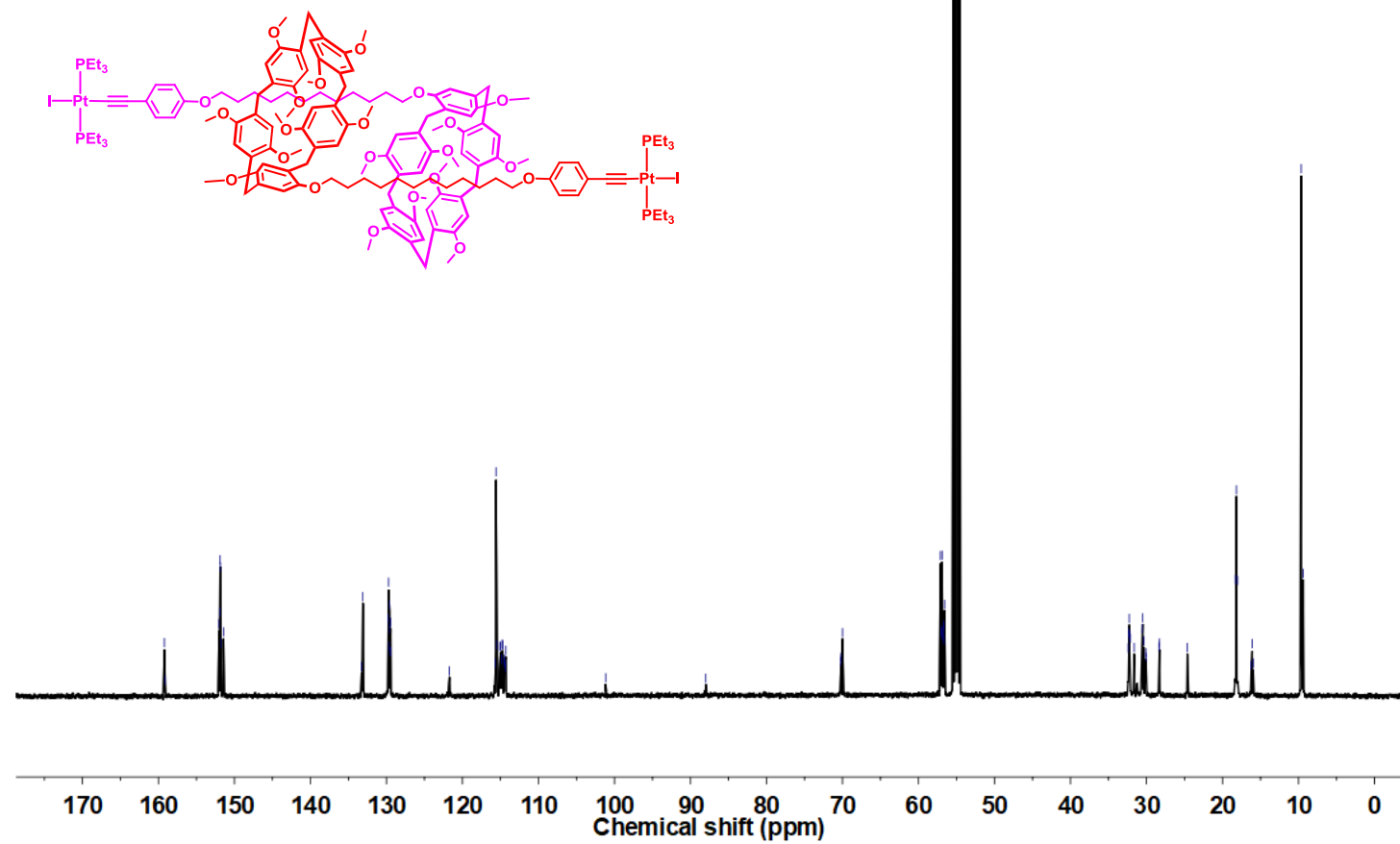

Figure S136: ${ }^{13} \mathrm{C}$ NMR spectrum $\left(\mathrm{CD}_{2} \mathrm{Cl}_{2}, 298 \mathrm{~K}, 126 \mathrm{MHz}\right)$ of model compound 2-a. 


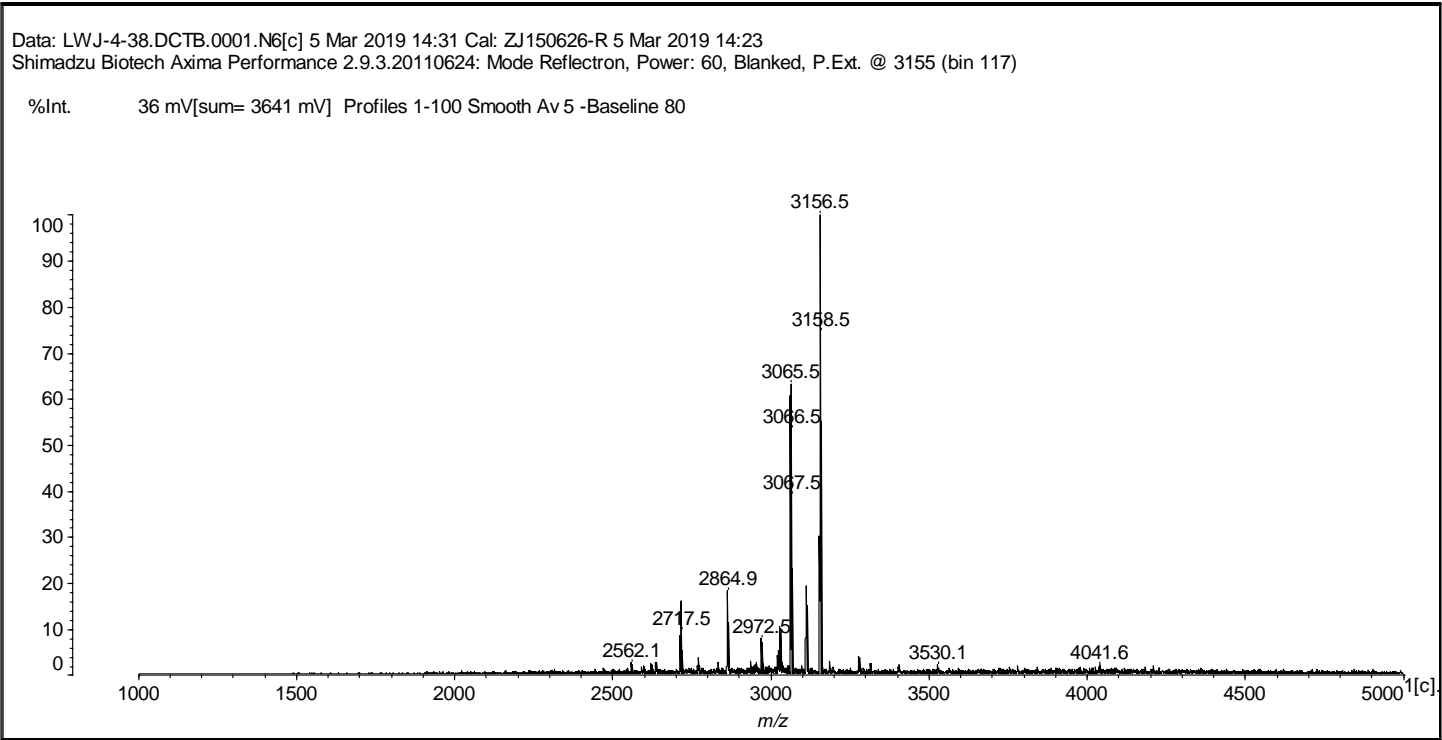

Figure S137: MALDI-TOF-MS spectrum of model compound 2-a.

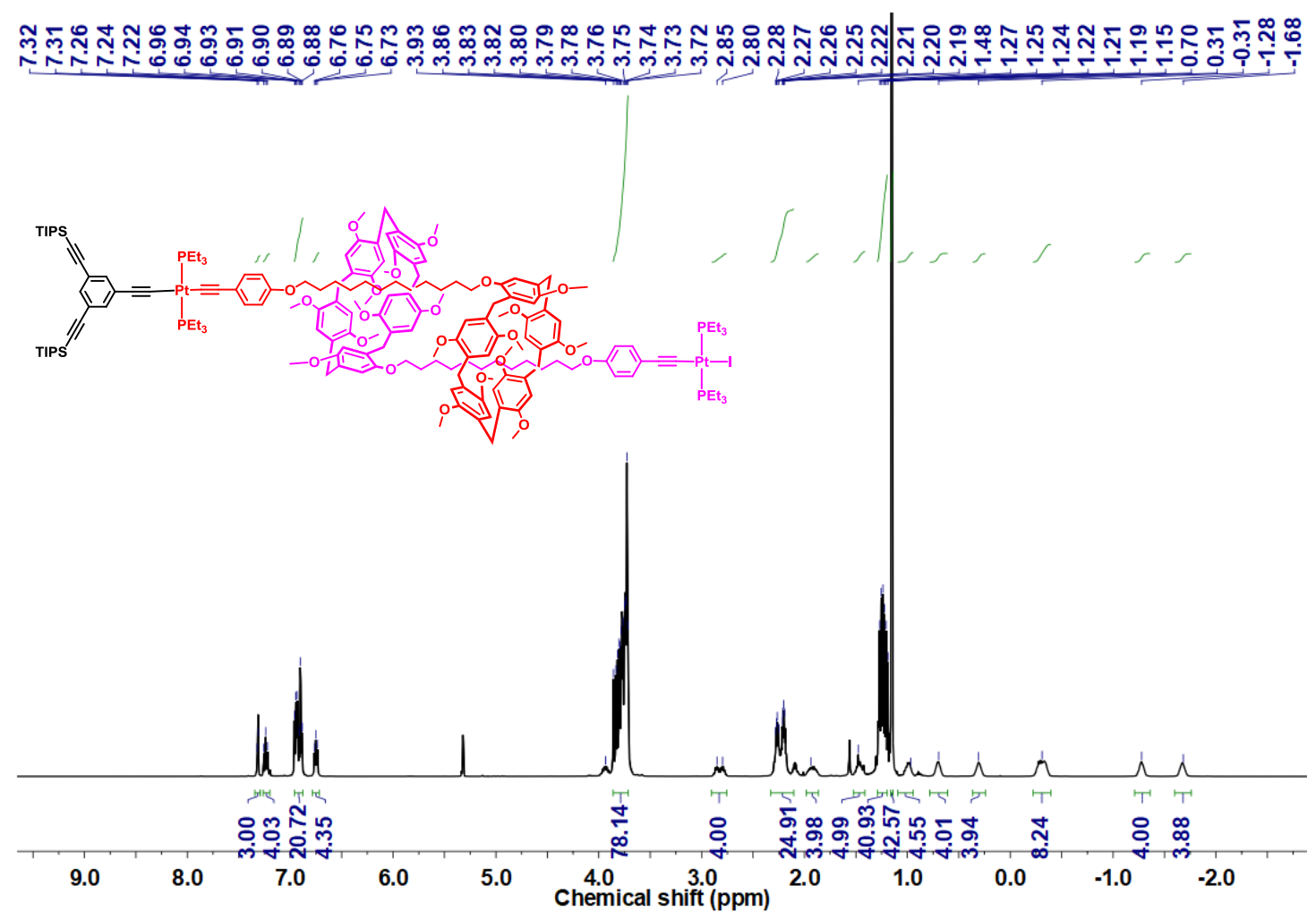

Figure S138: ${ }^{1} \mathrm{H}$ NMR spectrum $\left(\mathrm{CD}_{2} \mathrm{Cl}_{2}, 298 \mathrm{~K}, 500 \mathrm{MHz}\right)$ of model compound 1-a. 


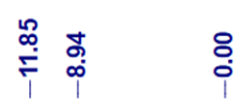
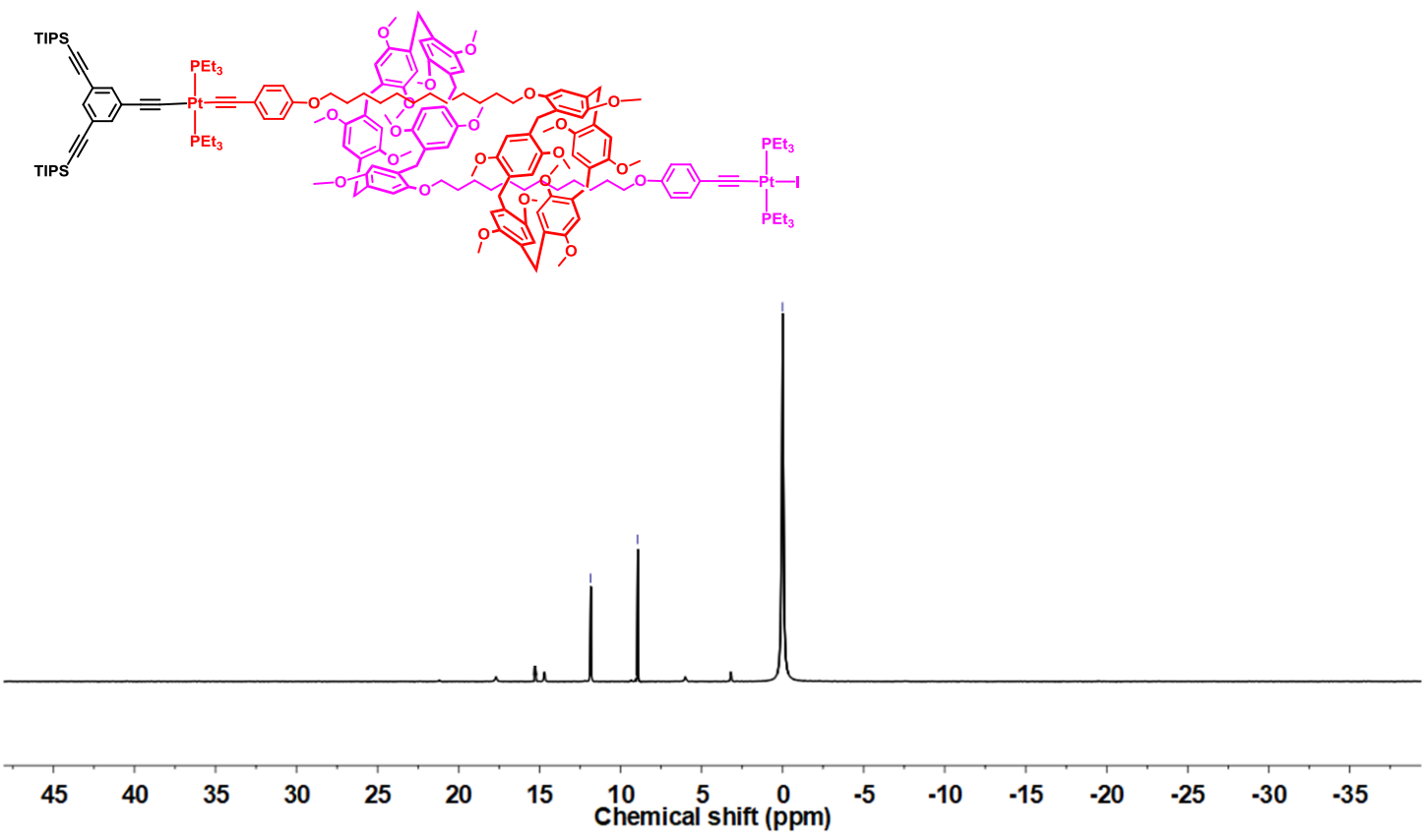

Figure S139: ${ }^{31} \mathrm{P}$ NMR spectrum $\left(\mathrm{CD}_{2} \mathrm{Cl}_{2}, 298 \mathrm{~K}, 202 \mathrm{MHz}\right)$ of model compound 1-a.

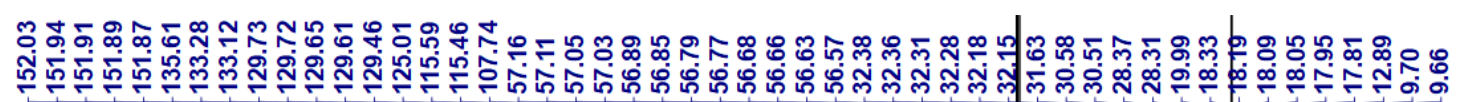
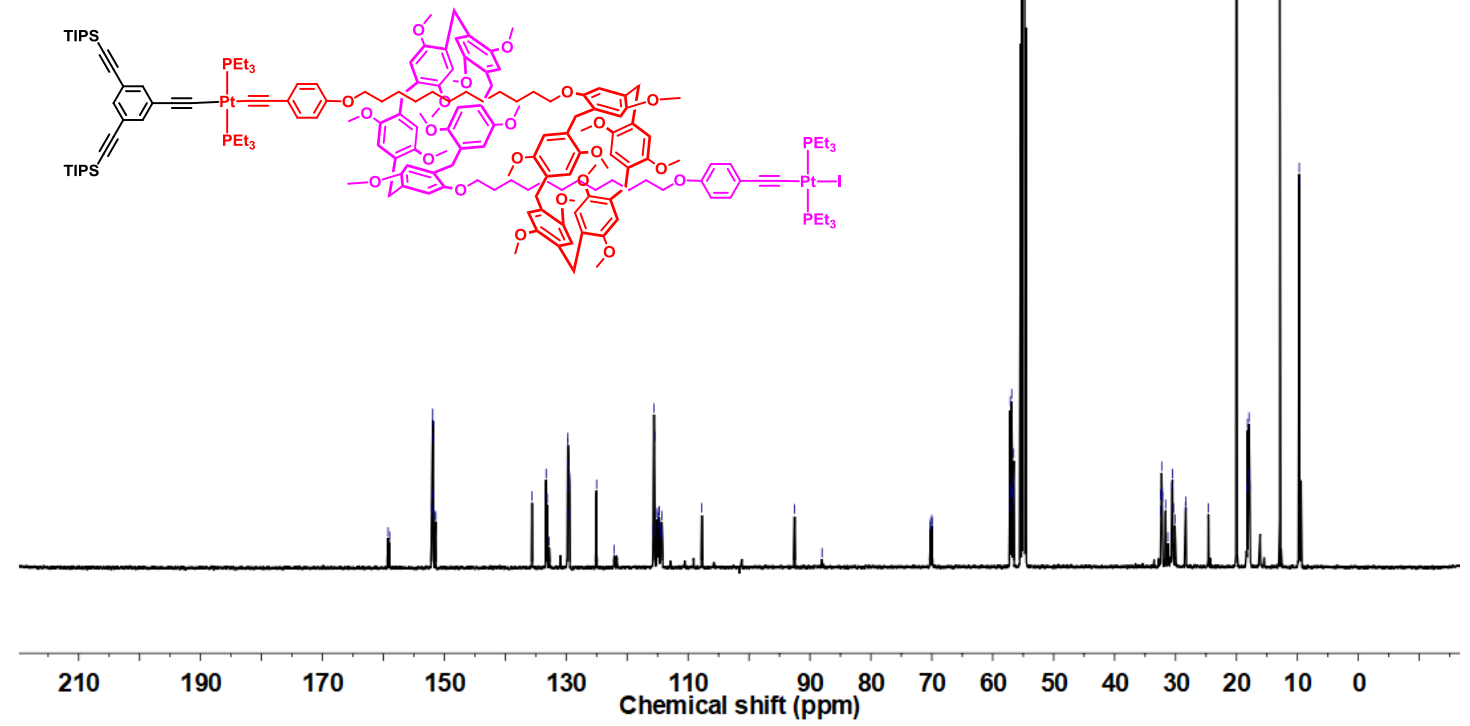

Figure S140: ${ }^{13} \mathrm{C}$ NMR spectrum $\left(\mathrm{CD}_{2} \mathrm{Cl}_{2}, 298 \mathrm{~K}, 126 \mathrm{MHz}\right)$ of model compound 1-a. 


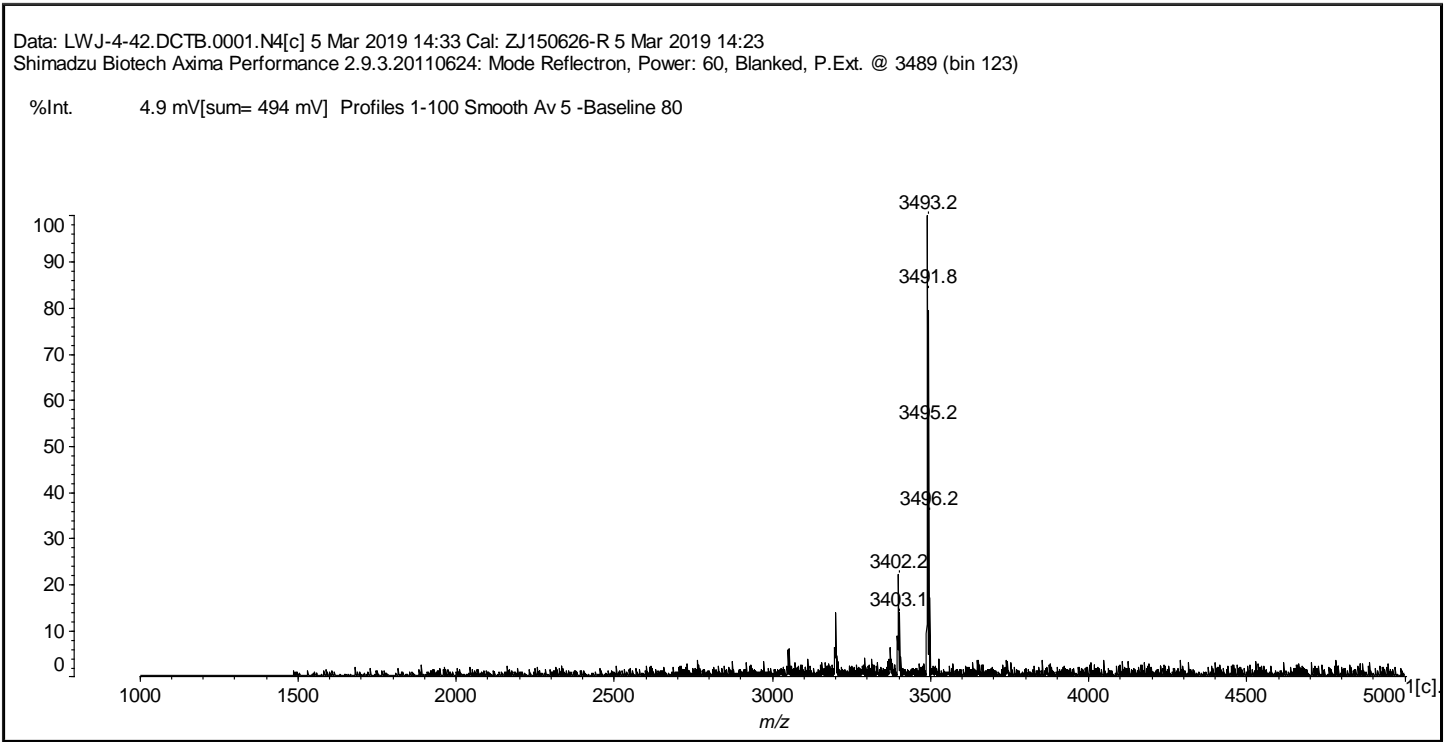

Figure S141: MALDI-TOF-MS spectrum of model compound 1-a.

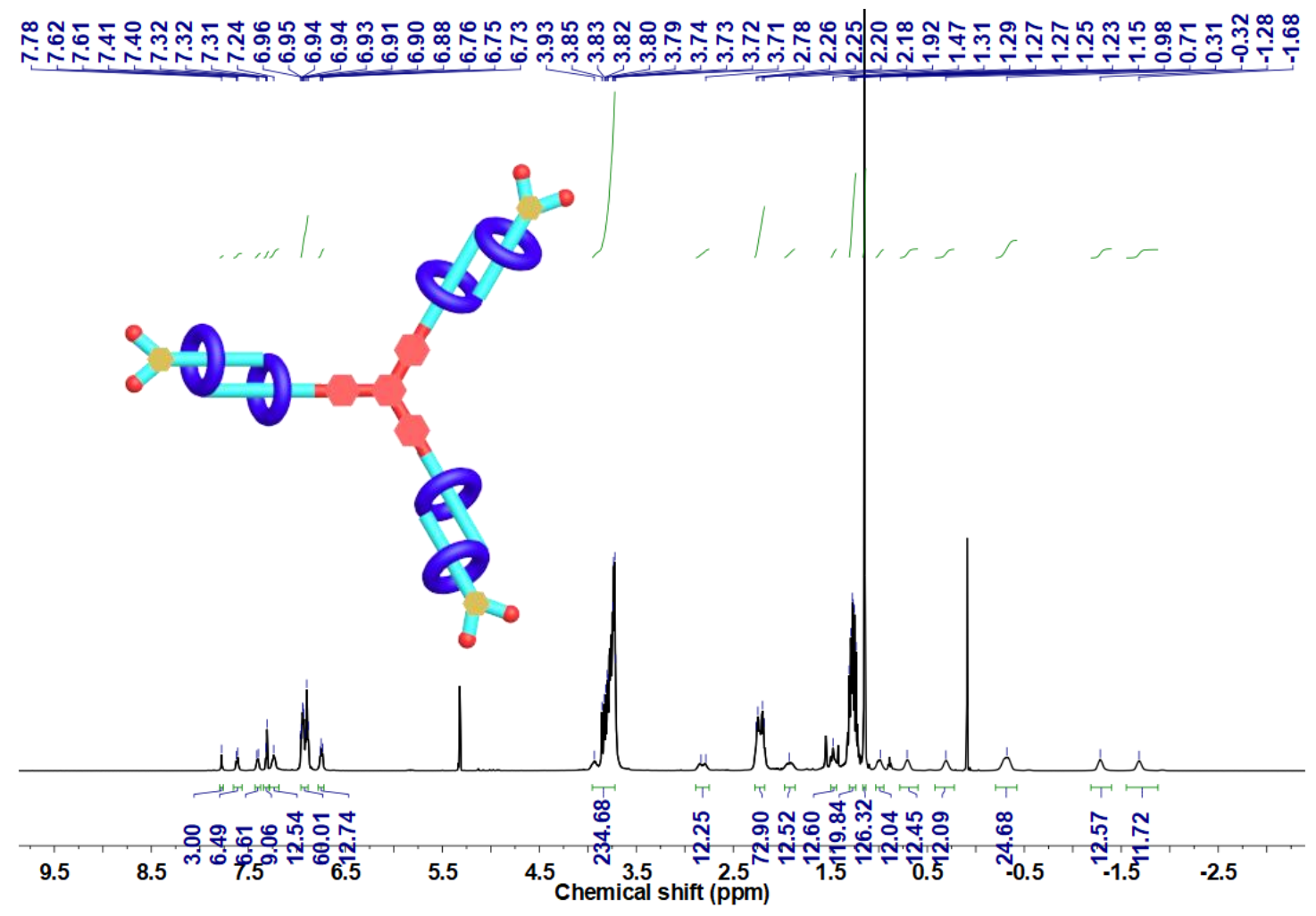

Figure S142: ${ }^{1} \mathrm{H}$ NMR spectrum $\left(\mathrm{CD}_{2} \mathrm{Cl}_{2}, 298 \mathrm{~K}, 500 \mathrm{MHz}\right)$ of model daisy chain dendrimer DC-G1-a. 


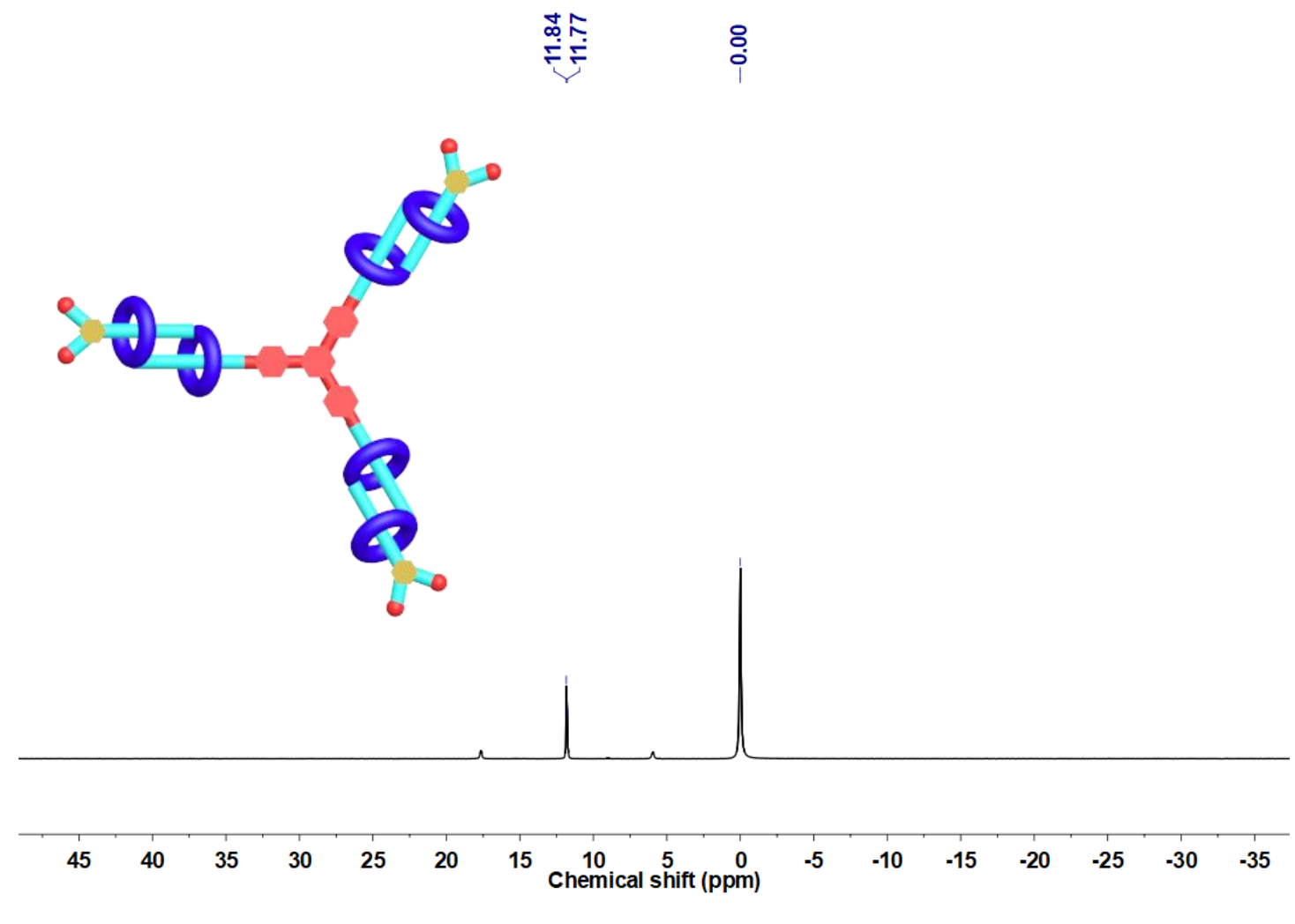

Figure S143: ${ }^{31} \mathrm{P}$ NMR spectrum $\left(\mathrm{CD}_{2} \mathrm{Cl}_{2}, 298 \mathrm{~K}, 202 \mathrm{MHz}\right)$ of model daisy chain dendrimer DC-G1-a.

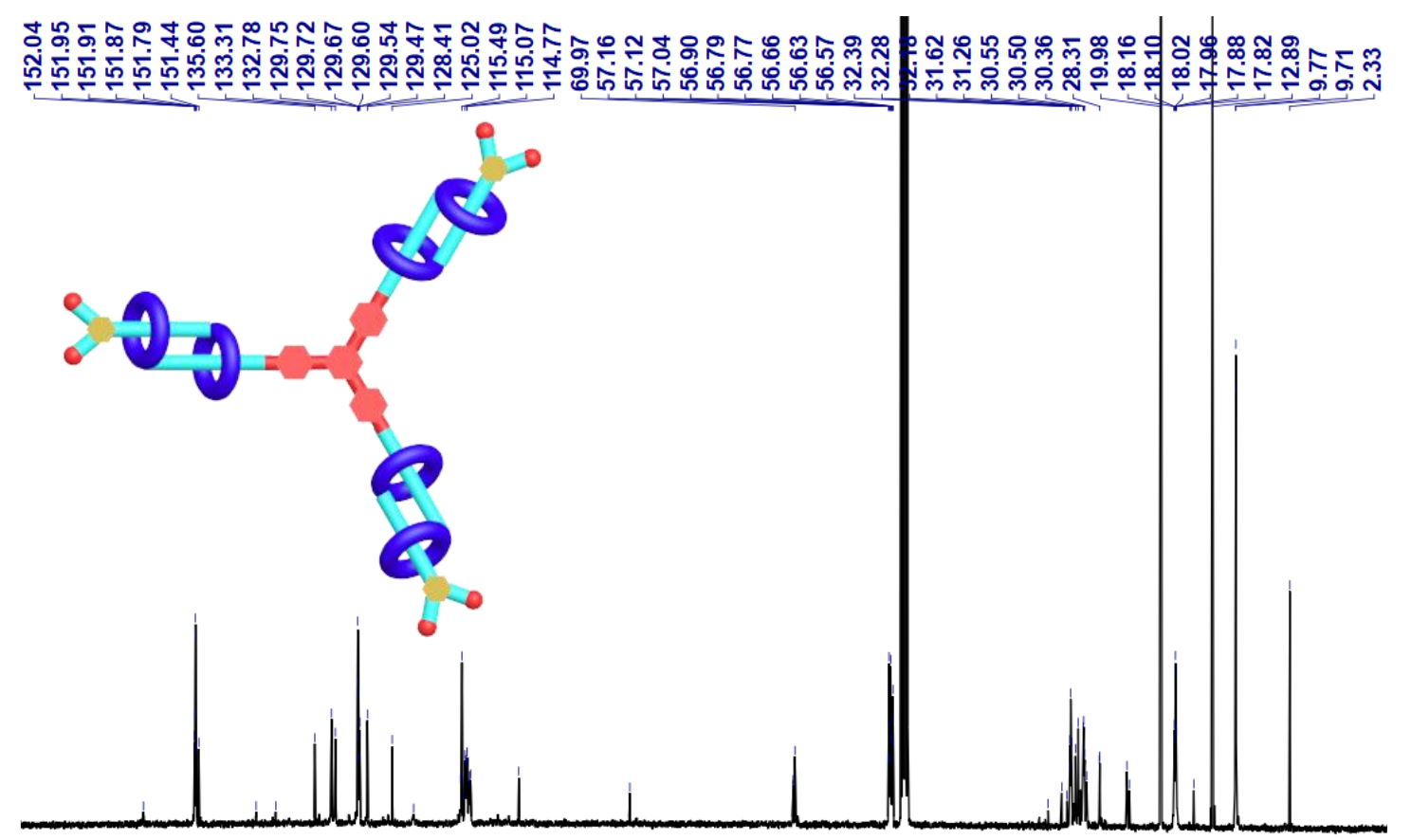

$\begin{array}{lllllllllllllllll}170 & 160 & 150 & 140 & 130 & 120 & 110 & 100 & \begin{array}{c}90 \\ \text { Chemical shift (ppm) }\end{array} & 60 & 50 & 40 & 30 & 20 & 10 & 0 & -11\end{array}$

Figure S144: ${ }^{13} \mathrm{C}$ NMR spectrum $\left(\mathrm{CD}_{2} \mathrm{Cl}_{2}, 298 \mathrm{~K}, 126 \mathrm{MHz}\right)$ of model daisy chain dendrimer DC-G1-a. 


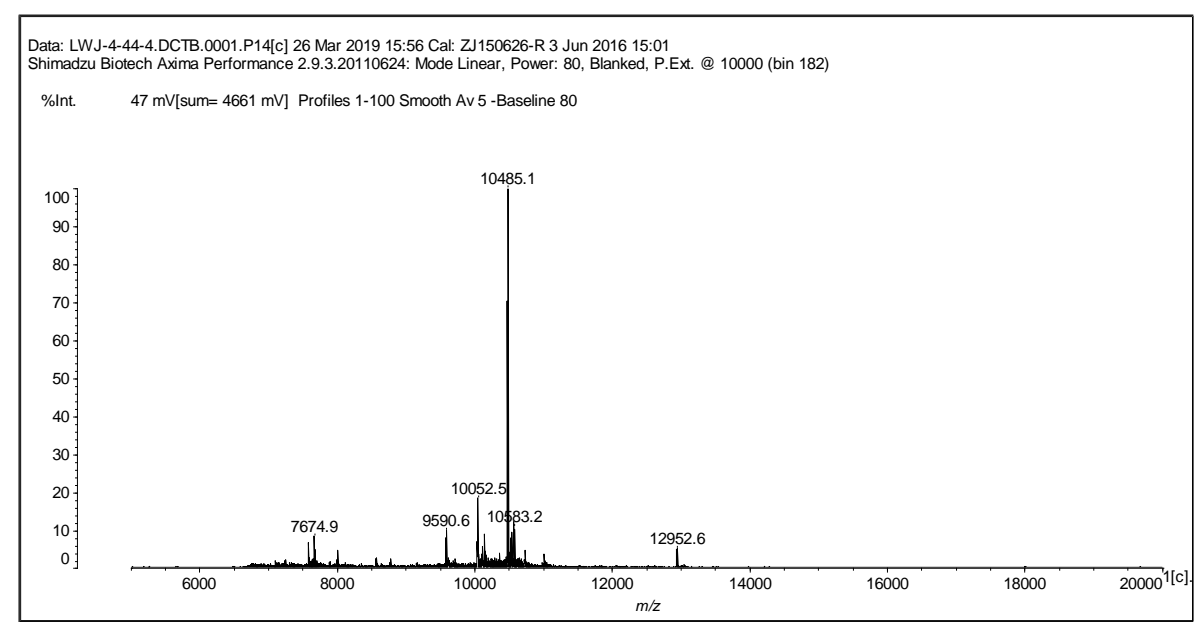

Figure S145: MALDI-TOF-MS spectrum of model daisy chain dendrimer DC-G1-a.

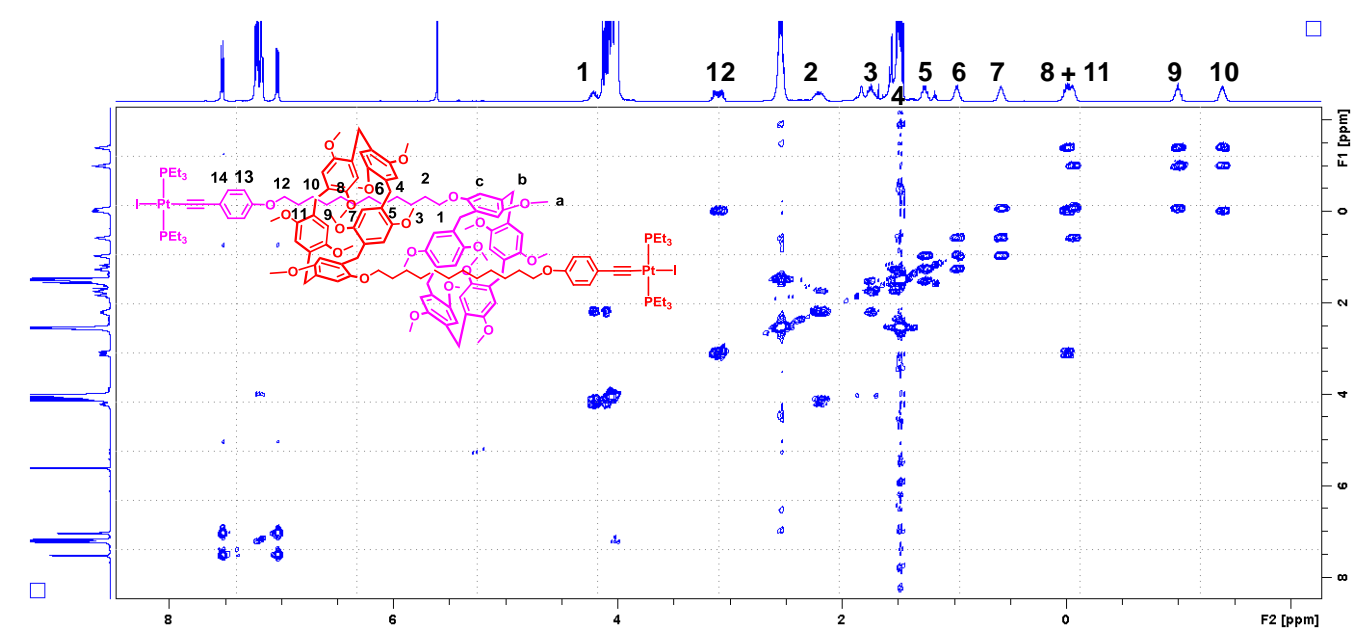

Figure S146: $2 \mathrm{D}{ }^{1} \mathrm{H}-{ }^{1} \mathrm{H}$ COSY spectrum $\left(\mathrm{CD}_{2} \mathrm{Cl}_{2}, 298 \mathrm{~K}, 500 \mathrm{MHz}\right)$ of the [c2]daisy chain rotaxane 2-a.

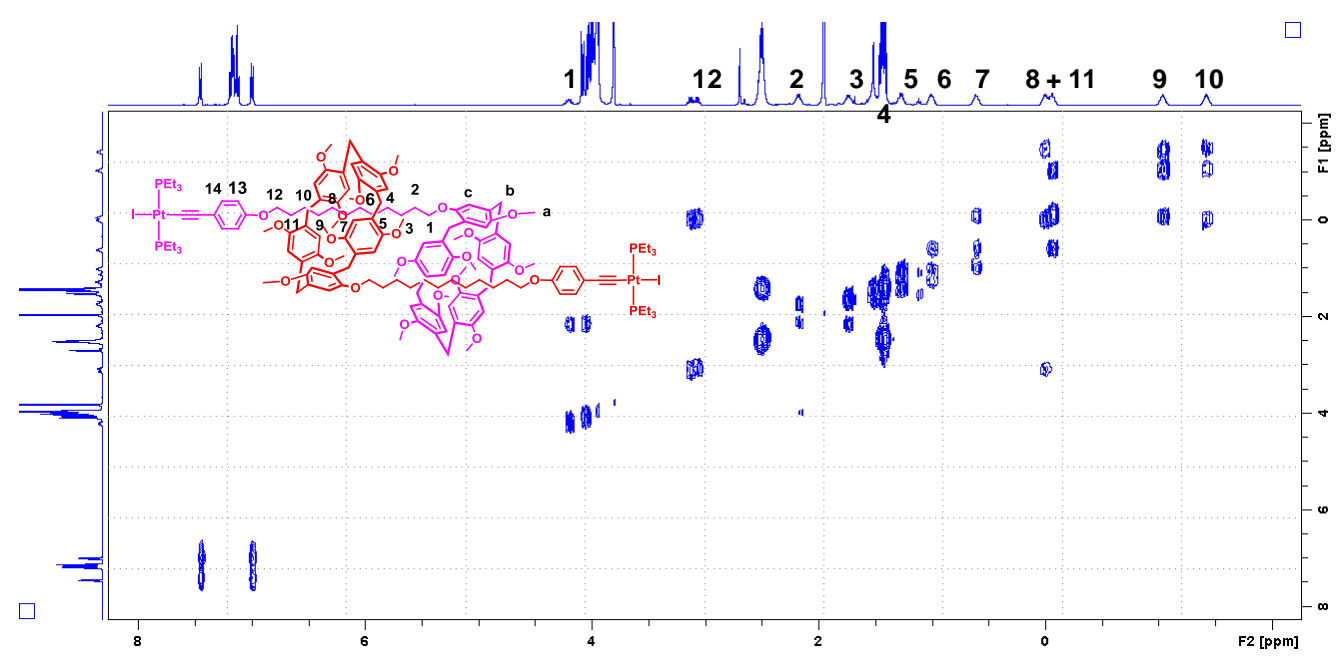

Figure S147: 2D ${ }^{1} \mathrm{H}-{ }^{1} \mathrm{H}$ COSY spectrum (THF- $d_{8}, 298 \mathrm{~K}, 500 \mathrm{MHz}$ ) of the [c2]daisy chain rotaxane 2 -a. 


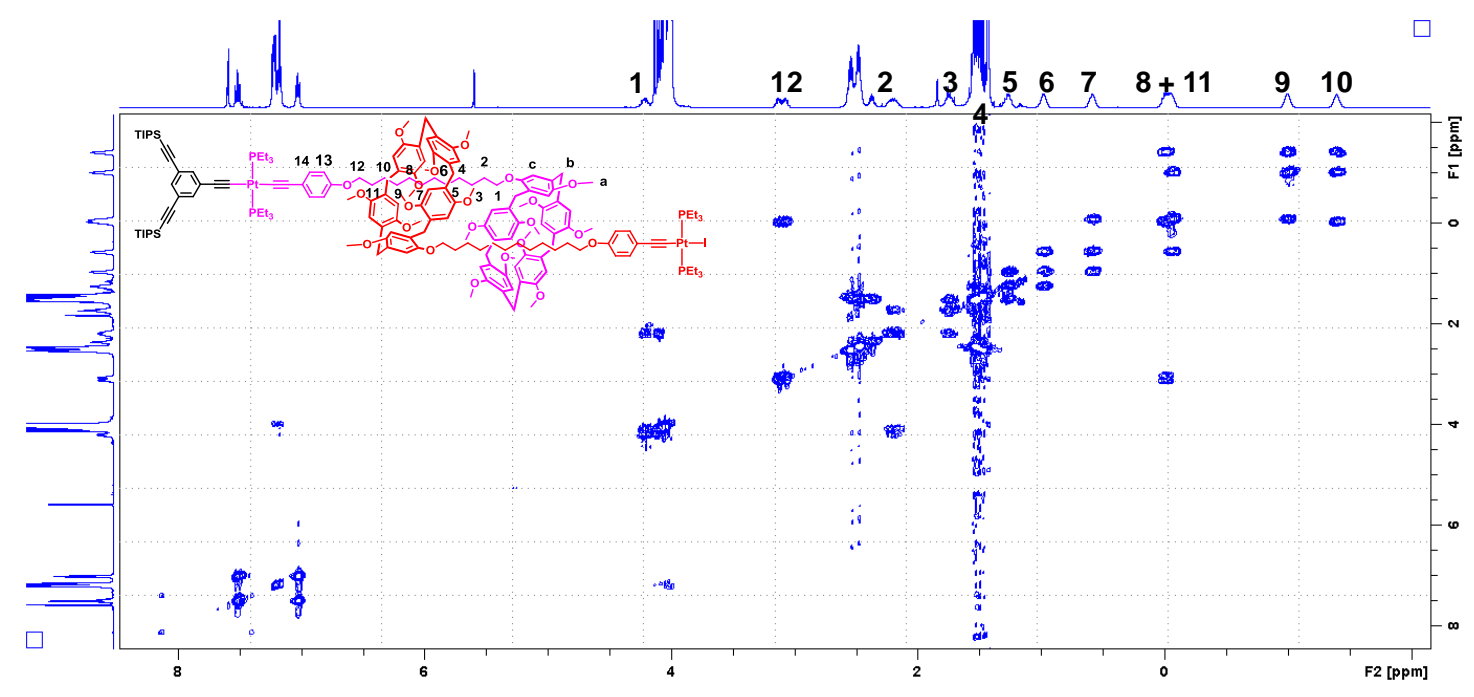

Figure S148: 2D ${ }^{1} \mathrm{H}-{ }^{1} \mathrm{H}$ COSY spectrum $\left(\mathrm{CD}_{2} \mathrm{Cl}_{2}, 298 \mathrm{~K}, 500 \mathrm{MHz}\right)$ of the [c2]daisy chain rotaxane 1-a.

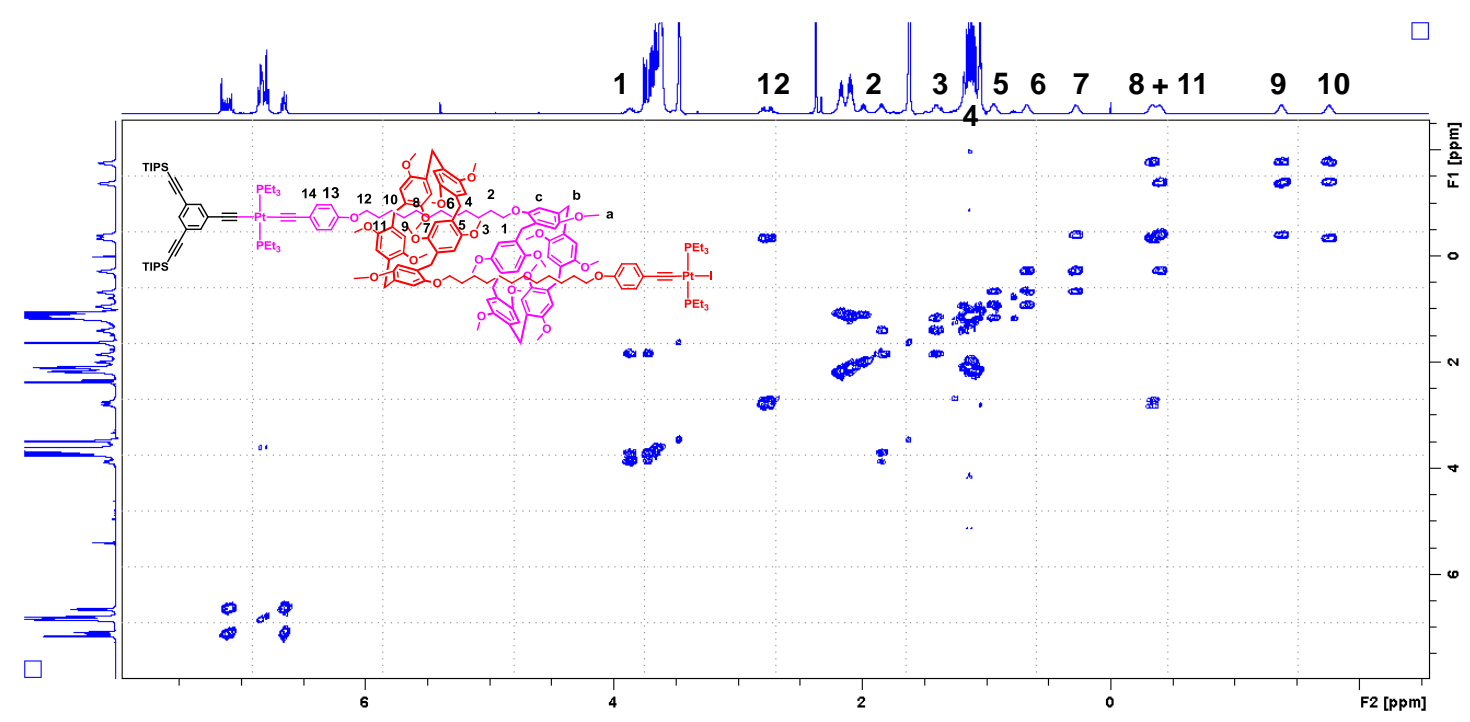

Figure S149: 2D ${ }^{1} \mathrm{H}-{ }^{1} \mathrm{H}$ COSY spectrum (THF- $d_{8}, 298 \mathrm{~K}, 500 \mathrm{MHz}$ ) of the [c2]daisy chain rotaxane 1-a. 


\section{Section 10. References}

1. F. Huang et al., Chem. Sci., 2012, 3, 3026-3031.

2. L. Wang et al., Org. Biomol. Chem., 2014, 12, 1079-1089.

3. T. Wei et al., Chin. J. Chem., 2016, 34, 1263-1267.

4. H.-B. Yang et al., Chem. Commun., 2018, 54, 2224-2227.

5. J. J. P. Stewart, J. Molec. Model., 2013, 19, 1-32.

6. MOPAC2016, Version: 17.119L, J. J. P. Stewart, Stewart Computational Chemistry, web: $\underline{\text { HTTP://OpenMOPAC.net. }}$

7. S. Grimme et al., J. Chem. Phys., 2010, 132, 154104.

8. TURBOMOLE V7.1 2016, a development of University of Karlsruhe and Forschungszentrum Karlsruhe GmbH, 1989-2007, TURBOMOLE GmbH, since 2007; available from http://www.turbomole.com.

9. S. Grimme et al., J. Chem. Theory Comput., 2017, 13, 1989-2009.

10. Y. Ke et al., Neutron News 2018, 29, 14-17. 